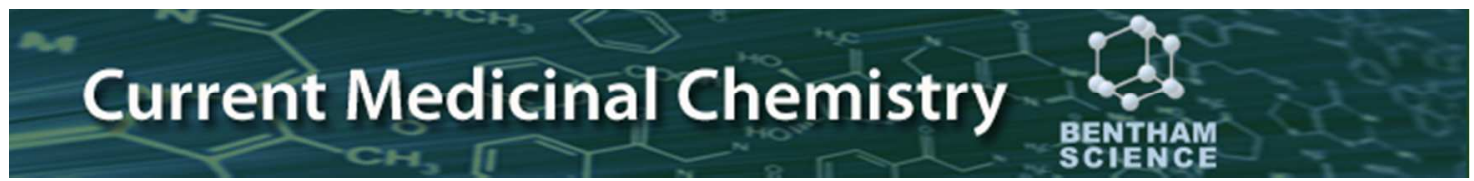

\title{
Medicinal Chemistry Updates on Bacterial Efflux Pump Modulators
}

\begin{tabular}{|c|c|}
\hline Journal: & Current Medicinal Chemistry \\
\hline Manuscript ID & Draft \\
\hline Manuscript Type: & Review \\
\hline Date Submitted by the Author: & $\mathrm{n} / \mathrm{a}$ \\
\hline Complete List of Authors: & $\begin{array}{l}\text { Durães, Fernando; Faculty of Pharmacy University of Porto } \\
\text { Pinto, Madalena; Faculty of Pharmacy University of Porto, Deptm Chemical } \\
\text { Sciences, Lab Organic and Medicinal Chemistry } \\
\text { Sousa, Maria Emília; Faculty of Pharmacy University of Porto, }\end{array}$ \\
\hline Keywords: & $\begin{array}{l}\text { Antimicrobial resistance, efflux pump inhibitors, natural products, synthetic } \\
\text { compounds, existing drugs, structure-activity relationship }\end{array}$ \\
\hline \multicolumn{2}{|c|}{$\begin{array}{l}\text { Note: The following files were submitted by the author for peer review, but cannot be converted to } \\
\text { PDF. You must view these files (e.g. movies) online. }\end{array}$} \\
\hline $\begin{array}{l}\text { Figure } 3 . c d x \\
\text { Figure } 5(1) \cdot c d x \\
\text { Figure } 5(2) \cdot c d x \\
\text { Figure } 5(3) \cdot c d x \\
\text { Figure } 5(4) \cdot c d x \\
\text { Figure } 5(5) \cdot c d x \\
\text { Figure } 5(6) . c d x \\
\text { Figure } 13(1) \cdot c d x \\
\text { Figure } 13(2) \cdot c d x \\
\text { Figure } 13(3) \cdot c d x \\
\text { Figure } 17 . c d x \\
\text { Figure } 19 . c d x\end{array}$ & \\
\hline
\end{tabular}




\title{
Medicinal Chemistry Updates on Bacterial Efflux Pump Modulators
}

\begin{abstract}
Antibiotic resistance is one of the most pressing health issues of our days. It can arise due to a multiplicity of factors, such as target modification, decrease in the drug uptake, changes in the metabolic pathways and activation of efflux pumps. The overexpression of efflux pumps is responsible for the extrusion of drugs, making antibiotic therapy fail, as the quantity of intracellular antibiotic is not enough to provide the desired therapeutic effect.

Efflux pumps can be included in five families according to their composition, nature of substrates, energy source, and number of transmembrane spanning regions. The ABC superfamily are mainly found in Gram-positive bacteria, use ATP as an energy source, and only a limited number of $\mathrm{ABC}$ pumps confer multidrug resistance (MDR).

On the other hand, the MFS family, most present in Gram-positive bacteria, and the RND family, characteristic of Gramnegative bacteria, are the most associated with antibiotic resistance. A wide variety of inhibitors have been disclosed for both families, from either natural or synthetic sources, or even drugs that are currently in therapy for other diseases.

The other two families are the SMR, which are the smallest drug efflux proteins known, and the MATE family, whose pumps can also resort to the sodium gradient as an energy source.

In this review, it is intended to present a comprehensive review of the classes of efflux pump inhibitors from the various sources, highlighting their structure-activity relationships, which can be useful for medicinal chemists in the pursuit of novel efflux pump inhibitors.
\end{abstract}

Keywords: Antimicrobial resistance; efflux pump inhibitors; natural products; synthetic compounds; existing drugs; structure-activity relationship.

\section{INTRODUCTION}

Bacteria were discovered the late $19^{\text {th }}$ century as infectious agents. It was, however, half a century later that antibiotics completely revolutionized medicine and became a turning point in human history. Unfortunately, the misruled use of these drugs led to the appearance of resistant strains, and to the concern of a return to the pre-antibiotic era, where the simplest, most treatable infections can become deadly [1-2]. Antibiotic resistance can arise due to multiple causes. The most common are the overuse and/or inappropriate prescribing, as well as extensive veterinary and agricultural use. These factors are especially alarming if it is taken into account the very few new antibiotics that were discovered in the past years [3]. Due to their genetic plasticity, bacteria can develop defense mechanisms in response to environmental threats. As far as antibiotics are concerned, bacteria use two major strategies to adapt to these drugs, which are mutations in genes and the acquisition of resistance genes through horizontal gene transfer. The first strategy can be manifested through modification of the target, decrease in the drug uptake, activation of efflux pumps to extrude the drug or changes in metabolic pathways. The latter is related to the acquisition of external genetic material through transformation, transduction, or conjugation [4-5]. Recently, vesicle-mediated resistance has also been regarded as a mechanism of resistance acquisition [6].

Efflux pumps are ubiquitous in bacteria, and can be encoded by genes present either in the bacterial chromosomes or in plasmids [7-8]. They concern five families, according to their composition, substrates, energy sources, and number of transmembrane spanning regions: the adenosine triphosphate (ATP)-binding cassette (ABC) superfamily, the resistancenodulation-division family (RND), the major facilitator superfamily (MFS), the small multidrug resistance (SMR) family, and the multidrug and toxic compound extrusion (MATE) family (Figure 1) [7-12].

\footnotetext{
*Address correspondence to this author at the Department of Organic and Pharmaceutical Chemistry, Faculty of Pharmacy, University of Porto, P.O. Box: 4050-313, Porto, Portugal; Tel/Fax: +351 220428 689, +351 226093 390; E-mail: esousa@ff.up.pt

Efflux pumps can be further divided into two major families, the primary transporters, which hydrolyze ATP as a source of energy, as is the case of the $\mathrm{ABC}$ family, and secondary transporters, which use the proton or sodium gradient as a source of energy [13]. Overexpression of multidrug efflux pumps is correlated with an increase in the minimum inhibitory concentration (MIC) of antibiotics, and thus, these pumps can be considered potential antibacterial targets.

Therefore, the development of efflux pump inhibitors may be a way of fighting against resistant microorganisms. Studies have been carried out on the structure of efflux pumps, as well as on their mechanisms of binding and extrusion, leading to the possibility of structure-based design of efflux pump inhibitors (EPIs). The rationale for the development of these EPIs could be, for instance, the inhibition of the energy sources required for the ability to extrude antibiotics, and the development of compounds that compete with the antibiotics for their extrusion. However,
} 
the main challenge lies in the fact that many compounds that present EPI activity and are already used for the treatment of other diseases are toxic for human cells in the concentrations required to present EPI activity and, therefore, clinically useless [7-9, 13].

Even though there are already several reviews which describe different classes of EPIs [11, 13-31], most focus on a particular source of compounds or on a class of pumps or bacteria. Herein, it is intended to present a comprehensive review of the several classes of EPIs from natural and synthetic sources and from existing drugs in therapeutics and to highlight structure-activity relationship studies in the referred efflux pumps (ABC, RND, MFS, SMR and MATE), which can be useful for medicinal chemists in the pursuit of novel compounds for this goal.

\section{2. $\mathbf{A B C}$}

The ABC transporters are primary transporters, and they exist in every membrane systems, whether the organism is prokaryotic or eukaryotic. P-Glycoprotein (P-gp) is a very well known human $\mathrm{ABC}$ transporter, involved in the resistance of cytotoxic drugs used in cancer therapy [32-34], whose modulation has been regarded as a study field [3537]. Structurally, an ABC transporter consists of four conserved domains, two transmembrane domains, and two cytoplasmic domains, which bind to nucleotides and, therefore, are involved in ATP binding $[32,38]$. In fact, the nucleotide binding domains are responsible for hydrolyzing ATP, which will lead to conformational changes in the attached transmembrane domains, making it possible for substrates to cross the lipid bilayer of the membrane, either in an inwards or an outwards fashion [39].

The bacterial transporters are highly selective for sugars, amino acids, metallic cations, organo-iron complexes, vitamins, and antibiotics [32, 40]. They are found in Grampositive bacteria, such as Enterococcus faecalis and Staphylococcus aureus, and confer resistance to macrolides and bacitracin. However, only a limited number of $\mathrm{ABC}$ pumps are associated with MDR, which means that $\mathrm{ABC}$ pumps do not play a pivotal role in conferring antibiotic resistance in bacteria [32-33].

As such, bacterial ABC transporter inhibitors are scarce. The few EPIs discovered in this context are mainly against MsrA (Figure 2, PDB 1NWA), whose tridimensional structure has been elucidated [41].

The structures of the $\mathrm{ABC}$ inhibitors that will be further detailed throughout this section are comprised in Figure 3.

\subsection{ABC inhibitors}

\subsubsection{Natural Products}

The search for natural products comes from the reason that plants produce antimicrobials to protect themselves from exogenous organisms. It is thought that plants can produce anti-MDR compounds to fight the microbial resistance phenomenon. Carnosic acid (1) was one of the major products isolated from the plant Rosmarinus officinalis. When tested against the macrolide resistant strain of $S$. aureus, expressing the MsrA pump, compound 1 displayed favorable results, showing an impressive 8-fold potentiation of erythromycin [42].

An extract of Lycopus europaeus led to the isolation of two new isopimarane diterpenes, methyl-1 $\alpha$-acetoxy- $7 \alpha, 14 \alpha$ dihydroxy-8,15-isopimarandien-18-oate (2) and methyl$1 \alpha, 14 \alpha$ - diacetoxy-7 $\alpha$-hydroxy-8,15-isopimarandien-18-oate (3), which showed no antibacterial activity, but were able to reduce erythromycin's MIC from $256 \mu \mathrm{g} / \mathrm{ml}$ to $128 \mu \mathrm{g} / \mathrm{ml}$, therefore causing a 2-fold potentiation. This behavior was similar to other isopimarane diterpenes isolated in this study, as well as for 5,9-dihydroxygeranyllinalool (4) and 9acetoxy-5-hydroxygeranyllinalool (5), two diterpenoids [4344].

Phytol (6) is an acyclic isoprenoid, and its EPI activity against the $\mathrm{ABC}$ pumps of $E$. coli was assessed in combination with nalidixic acid. Additionally, 15 derivatives were synthesized. One derivative in particular, compound 7 , was able to decrease the MIC of nalidixic acid by 16-fold, and four other derivatives reduced the MIC by 8 -fold. This effect was caused by the inhibition of ATP-dependent efflux pump and down-regulation of the efflux gene. In silico absorption, distribution, metabolism, and excretion (ADME) analysis was performed, taking into account parameters such as solubility in water, human intestinal absorption, plasma protein binding, blood-brain barrier penetration, hepatotoxicity and cytochrome P450 (CYP450) inhibition. Results showed all five active derivatives presented low permeability into the central nervous system and displayed moderate intestinal absorption. In terms of plasma protein binding level, all five compounds exhibited levels below $90 \%$, suggesting favorable distribution of the compounds into the blood. They also did not present hepatotoxicity, unlike reserpine, making them good lead compounds [45].

Ursolic acid (8) is a pentacyclic triterpenoid isolated from diverse natural sources. Its wide activity includes antiinflammatory, antimicrobial, diuretic, and antitumor activity, among others. When tested against Escherichia coli strains that were resistant and susceptible to nalidixic acid, ursolic acid did not display intrinsic antibacterial activity. However, ursolic acid (8) was able to reduce the MIC of nalidixic acid by 2 -fold, when both drugs were used in combination. This synergy led to further research on this scaffold. The first derivative prepared was the 3-O-acetyl ursolic acid, and showed a 4-fold decrease in the MIC of nalidixic acid. The lipophilic nature of this derivative, allied with the increased potency, led to the synthesis of other lipophilic ester derivatives. The methyl and ethyl esters of 3-O-acetyl ursolic acid did not show any increment in the activity. However, a further increase in the ester carbon chain increased the drug reversal potential by 2 -fold, in comparison to 3 - $O$-acetyl ursolic acid, against the resistant strain. This is the case of the isopropyl (9) and the $n$-butyl (10) derivatives. The addition of a pentyl group led to a decrease in activity. Furthermore, ursolic acid (8), and derivatives 9 and 10, reduced the MIC of tetracycline by 2 -fold, 8 -fold, and 8 -fold, 
respectively. When these compounds were tested against ATP-dependent efflux pumps, it was found that derivatives 9 and 10 were successful in inhibiting these pumps in terms of the liberated inorganic phosphate. Molecular modeling studies with the YojI pump, using maltose/maltodextrin transport ATP-binding protein as a homology model, showed that 9 was bound to the nucleophilic Ser-78, to the aromatic Phe-81, Trp-85 and Leu-86, the acidic Glu-94 and Glu-144 and the basic Ala-79, Ala-139, Ala-140, Ala-141, Ala-143, Arg-74, Arg-122, Arg-146, Leu-107, Val-80, Lys-96, and Ile-123 in the nucleotide binding domain. Derivative 10 was also subjected to the same kind of studies using the same model, and was predicted to bind in the same region [46].

A study with flavones and isoflavones from the plant Sophora moorcroftiana revealed that diosmetin (11) was able to inhibit three drug resistant strains of $S$. aureus, including the $\mathrm{ABC}$ family MsrA overexpressing strain, potentiating the effects of norfloxacin and streptomycin [47]. These results corroborate the works of Chan et al (2013), who had previously studied the effects of diosmetin (11) as an EPI [48]. This group observed a synergistic effect of this flavonoid with erythromycin, which was 5-fold greater than that of verapamil [48].

4-Hydroxy- $\alpha$-tetralone (12) isolated from the plant Ammannia multiflora, showed enhancement against nalidixic acid resistant strains of Escherichia coli, prompting the synthesis of derivatives in order to infer their mechanism of action. Five derivatives were synthesized, and it was shown that compound $\mathbf{1 3}$ was able to inhibit the expression of the $\mathrm{ABC}$ transporter protein gene yojI, which encodes the $\mathrm{ABC}$ protein YojI, and was successful in combination with tetracycline [49]. Molecular modelling studies with compound $\mathbf{1 3}$ and a homology model of the YojI protein revealed the following bound residues: Ser-78 (nucleophilic), Leu-86, Ile-123, Ala-137, Ala-140, Ala-141, and Ala-143 (hydrophobic), Glu-144 (acidic), Arg-122 (basic), Phe-81 (aromatic), Glu-144 (acidic), Arg-146 and Lys-96 (basic), and Trp-85 (hydrophobic), the same region as noted for ursolic acid derivatives 9 and 10. Compound 13 showed good affinity and strong hydrophobic interaction with the YojI protein, corroborating the fact that this compound acts by the inhibition of ATP dependent efflux pumps [49].

The plant Portulaca oleraceae is used in folk medicine for its anti-inflammatory, anti-septic and diuretic effects, among others. A study of its fatty acids demonstrated that linoleic (14) and oleic acids (15) had synergistic activity with macrolides for the MsrA overexpressing strain of methicillin-resistant $S$. aureus (MRSA). In fact, erythromycin's activity was enhanced when in combination with either of these two compounds, likely through the inhibition of the MsrA ATP dependent pump. Since these fatty acids are essential for humans, low toxicity is expected, making them promising agents to use in combination with antibiotics [50].

Lysergol (16) is a clavine alkaloid of the ergoline family. Recently, its EPI activity was studied, and derivatives were synthesized [51]. The compounds were tested against susceptible and resistant to nalidixic acid strains of $E$. coli and a tetracycline resistant strain. While not possessing antibacterial activity, lysergol (16) showed synergy with nalidixic acid at the concentration of $10 \mu \mathrm{g} / \mathrm{ml}$, reducing its MIC by 8-fold in the resistant strain of $E$. coli and 4-fold in the susceptible strain. Of the thirteen semi-synthetic derivatives prepared, derivatives $\mathbf{1 7}$ and $\mathbf{1 8}$ reduced nalidixic acid's MIC by 8 -fold, twice as much as lysergol (16), in the susceptible strain. A 4-fold potentiation of nalidixic acid was observed for the three compounds when tested against the resistant strain. As for the tetracycline resistant strain, all three compounds were able to reduce tetracycline's MIC by 8 -fold. Further studies were performed, in order to conclude whether or not these compounds were capable of interfering with ATP-dependent efflux pumps, and it was proven that all three compounds inhibited ATPase activity, concerning the liberation of inorganic phosphate. A down-regulation of the gene yojI was also observed when the tetracycline resistant strain was treated with lysergol (16) and its two most potent derivatives, $\mathbf{1 7}$ and $\mathbf{1 8}$, which further confirms the activity of these compounds as ABC transporter inhibitors [51].

\subsubsection{Existing Drugs}

As part of a screening program for P-gp inhibitors, GG918 (19) was discovered. This synthetic compound had already showed success in increasing the intracellular concentration of paclitaxel in cancer cells. In S. aureus, this compound was able to slightly potentiate the activity of norfloxacin in a strain overexpressing the ABC efflux pump MsrA, suggesting that it may possess minimal activity over this pump [52].

The human P-gp inhibitors, vanadates, have been studied as inhibitors of the MutS pumps, belonging to the ABC ATPase superfamily. In fact, these compounds have demonstrated activity against the MutS in $P$. aeruginosa and $E$. coli. Decavanadate was the most effective, followed by orthovanadate (20), and they act non-competitively, possibly by replacing the inorganic phosphate. The fact that the $\mathrm{ABC}$ ATPases inhibited by orthovanadate are phosphorylated leads to the assumption that the low inhibitory effect could be attributed to the absence of a stable phosphorylation state of MutS [53].

\section{MFS}

The MFS is the most extensively studied family of transporters, present in both Gram-positive and Gramnegative bacteria, and comprises uniporters, symporters and antiporters. It also constitutes the largest family of secondary transporters, with over 10000 sequenced members. Its substrates are diverse, and can range from ions, to carbohydrates, lipids, amino acids, nucleosides, among others. As many members of the MFS originate bacterial MDR, these transporters can pose as a good system for modulation studies [7, 19, 38, 54].

With the elucidation of the crystal structure of the MFS pump EmrD of E. coli (Figure 4, PDB 2GFP) [55], a general 
structure could be proposed for MFS transporters: twelve transmembrane helices that form a compact structure, with four of these transmembrane helices facing away from the interior cavity and the rest forming the internal cavity, constituted mostly by hydrophobic residues [7].

The efflux of drugs is only possible if the carrier alternates between two conformations, so that the substrate can bind to the convenient site in either side of the membrane. However, a limitation concerning the energy involved to displace the exposed hydrophilic surface of a protein can be inferred. It has been proved that either the oligomerization domain or the transport subunit provide the support needed to the movement of the substrate binding site, through a rigid-body rotation of the substrate binding domain, referred to as the "elevator mechanism" [56-57]. For this family, the most extensively studied efflux pump is the NorA pump of $S$. aureus.

The inhibitors that have proven activity against efflux pumps of the MFS family are gathered in Figure 5.

\subsection{MFS inhibitors}

\subsubsection{Natural Products and Derivatives}

Terpenes and terpenoids, existent in the essential oils of plants, have showed promising therapeutic potential in various fields. Therefore, studies about EPI potential of these compounds were carried out. Monoterpenes nerol (21), estragole (22), and dimethyl-octanol (23) were tested against $S$. aureus. Compounds $\mathbf{2 1}$ and $\mathbf{2 3}$ were able to enhance the activity of norfloxacin, decreasing its MIC against the NorA overexpressing strain while not displaying intrinsic antibacterial activity. These open chain terpenes proved to be more effective than 22, which carried a benzene ring, at inhibiting the NorA pumps [58].

Citronellal (24) and citral (25) are monoterpenes, and were chosen as starting material for the synthesis of new amide derivatives. The basis for this study was the fact that aromatic amides are recognized as potent EPIs, whereas alkyl, alkenyl, and alkynil amides had not yet been explored. The compounds obtained were 5,9-dimethyldeca-2,4,8trienoic acid amides and 5,9-dimethyldeca-2,8-dienoic acid amides, whose activity for the potentiation of ciprofloxacin in $S$. aureus was assessed. Of the first 22 synthesized compounds, seven were able to reduce ciprofloxacin's MIC by 4 -fold. Further modifications were performed, and the C9 methyl group was replaced by an amide, yielding compounds with similar potency as the previous ones. Derivatives subjected to hydrogenation led to decreased activity, which reveals that unsaturation is an important feature for antimicrobial potentiation. The mechanism of inhibition was assessed through ethidium bromide efflux, confirming these compounds act by inhibiting NorA, being compound 26, a 9-formyl-5-methyldeca-2,4,8-trienoic acid amide, the most potent inhibitor [59].

Ferruginol (27), a diterpene, showed efficiency in inhibiting the efflux of ethidium bromide, proving its activity as a NorA inhibitor. It also showed a decrease in norfloxacin efflux in $S$. aureus [60]. Totarol (28), a phenolic diterpene, also showed a reduction in ethidium bromide efflux, suggesting activity against NorA pump, even though its mechanism is not yet known [61].

The triterpene ginsenoside $20(S)$-Rh2 (29), a trace compound from red ginseng, also enhanced the effect of ciprofloxacin against $S$. aureus, through a NorA-mediated efflux inhibition, promoting the antibiotic accumulation in the bacteria [62].

Reserpine (30) is a natural alkaloid derived from Rauwolfia vomitoria, known as an anti-hypertensive agent, with inhibitory activity against human P-gp and breast cancer resistance protein (BCRP) [63-64]. Reserpine (30) has proven to be an effective inhibitor of the Bmr multidrug transporter in Bacillus subtilis, diminishing the efflux of ethidium bromide. Its structural analogue rescinnamine also had EPI activity, in a smaller extension than reserpine (30) [65]. Through DNA sequencing of the $b m r$ genes of mutants, it was shown that reserpine (30) interacts with the residues Phe-143, Val-286, and Phe-306 of Bmr pumps, inhibiting drug transport [19]. Compound $\mathbf{3 0}$ has shown potential as an EPI for the inhibition of NorA efflux pumps in $S$. aureus, which are structurally related to Bmr pumps, reversing susceptibility to fluoroquinolones, demonstrating reductions up to 4-fold in MIC for some tested strains. It has been shown that 30's effect is more pronounced in hydrophilic fluoroquinolones, such as ciprofloxacin [66]. The mechanism by which $\mathbf{3 0}$ performs the inhibitory activity is through direct binding to the transporters that mediate drug efflux, acting as a competitive inhibitor [19, 67]. Reserpine (30) also succeeded in inhibiting the chloramphenicol efflux by CmlR1 and CmlR2 of Streptomyces coelicolor, the ciprofloxacin and norfloxacin efflux in Listeria monocytogenes's pump Lde, and the kanamycin and fusidic acid efflux by LmrS from a MRSA clinical isolate. On the other hand, for L. monocytogenes, 30 was not able to reverse susceptibility to linezolid and lincomycin, which suggests that 30 does not completely overlap with the structure binding sites of efflux pumps, being the inhibition dependent on the type and nature of the substrate [19]. However, reserpine (30) shows neurotoxicity at the concentration required to inhibit NorA, leading to the need to investigate new inhibitors [67].

Piperine (31), an alkaloid found in the fruits of Piper nigrum, was found to be an inhibitor of ciprofloxacin efflux in $S$. aureus, reducing the MIC by 2 -fold. It is thought that compound $\mathbf{3 1}$ acts as a direct inhibitor of drug efflux. Compound 31 inhibits drug transport of the pump MdeA, potentiating the effects of benzalkonium chloride, doxorubicin, daunorubicin, novobiocin, tetraphenylphosphonium chloride, rhodamine $6 \mathrm{G}$, virginiamycin and mupirocin [17, 19-20]; it also inhibits ciprofloxacin efflux by NorA [68].

Given these positive results obtained with piperine (31), further research into this scaffold was performed. Kumar et al. (2008) performed a screening of about 200 structurally diverse compounds for the inhibition of NorA efflux pump, choosing ethidium bromide as a substrate, since the only 
mechanism through which this compound is expelled is active transport (68). About 50 compounds were able to increase the intracellular concentration of ethidium bromide by 4 -fold, while not displaying antibacterial activity. Five of these compounds were more potent than piperine (31), being three of these compounds selected for further studies: 32, 33, and 34, being 32 and 34 more potent than 31 by 40-fold [18, 68]. Assumptions regarding structure-activity relationship (SAR) studies of these molecules could be drawn (Figure 6). It was demonstrated that the introduction of an alkyl group at C-4 position, mainly an isopropyl, isobutyl or di-isopropyl group, coupled with the substitution of the piperidinyl moiety with an aromatic amine, such as aniline, would lead to maximum potentiation of ciprofloxacin; if the anilinyl moiety is substituted with a 2-hydroxymethyl group or a nitrile, regardless of the positional isomery, ciprofloxacin activity would increase. Replacing the piperidinyl moiety with a phenylacetamide yields good potentiation of ciprofloxacin, reducing its MIC by 4 -fold, regardless of the isomery. Unsaturation is crucial for the potentiation, as well as the amide. Lastly, the retention of 3,4methylenedioxyphenyl or 4-methoxyphenyl groups in the 1,3-benzodiol moiety also gives favorable results [68-70]. Plants of the Berberis species have the ability to produce the alkaloid berberine, which is a plant secondary metabolite produced in response to microbial invasion, whose activity is enhanced by a flavolignane also produced by these species, 5'-methoxyhydnocarpin (35) [71]. Compound 35 exists in a mixture of anion and neutral compound at physiological $\mathrm{pH}$, since it has a phenolic group with a $\mathrm{pKa}$ of 7.3. Due to the acidic properties of this 7-OH group, 35 is likely to be a specific microbial MDR inhibitor, as flavonoids with P-gp inhibitor properties usually possess alkylated 7- $O$ groups. In fact, when added to a wild type strain of $S$. aureus, compound 35 caused a decrease in norfloxacin's MIC to $0.25 \mu \mathrm{g} / \mathrm{ml}$. Furthermore, when tested with berberine and palmatine, 35 appeared to completely inhibit NorA, suggesting it acts as a non-competitive inhibitor [71].

A number of hydnocarpin-type flavolignanes were synthesized in order to perform SAR studies. Flavolignanes with and without phenolic groups at the 5 and 7 positions had comparable activity. Diosmetin (11), the parent compound, presents hydroxyl groups in the A ring and was the most potent compound. Peracetate derivatives of $\mathbf{3 5}$ have approximately the same potency as their parent compounds, and could be considered prodrugs, if deacetylated by $S$. aureus, which seems unlikely. Considering the D ring, compounds bearing a 3'"-methoxy-4"'-hydroxy moiety displayed good activity, and 3', 5 ',-dimethoxy-4"'-hydroxy moieties conferred a slightly less active compound. As for the $\mathrm{B}$ ring, the presence of an $\mathrm{H}$ or an $\mathrm{OH}$ is not critical for activity [72]. In addition, a flavone isolated from Alkanna orientalis, sarothrin (36), blocked ethidium bromide efflux by $S$. aureus, suggesting its potential activity as a NorA inhibitor [73].

Biochanin A (37), an isoflavone, also showed potentiation of norfloxacin and berberine against wild-type $S$. aureus and Bacillus megaterium. An analogue of luteolin, orobol (38), was quite active as a NorA inhibitor, unlike luteolin itself. It was shown that flavones which have a single methoxy in its B-ring are more potent than flavones with two methoxy groups in the B-ring [74].

Another flavone, baicalein (39), was capable of potentiating the effect of ciprofloxacin in $S$. aureus. Studies aimed at measuring the efflux of ciprofloxacin and pefloxacin, a poor NorA substrate, were performed, and showed that compound 39 was much more potent at restoring ciprofloxacin sensitivity than pefloxacin, leading to the conclusion that this flavone acts as a NorA efflux inhibitor, even though its mechanism remains unknown [75].

Maia et al. (2011) identified six flavonoids from the plant Praxelis clematidea: apigenine, genkwanin, 7,4'dimethylapigenin, trimethylapigenin, cirsmaritin, and tetramethylscutellarein (40) [76]. These methoxylated flavones showed no antibacterial activity, but were able to reduce the MIC of norfloxacin at least by 2 -fold and up to 16-fold, as was the case of $\mathbf{4 0}$, at a concentration of $8 \mu \mathrm{g} / \mathrm{ml}$, being therefore considered NorA efflux pump modulators. It is thought that the lipophilicity of the flavonoids, strengthened by the presence of methoxyl groups, is responsible for this activity [21]. Presence of a methoxy in the 4' position proved to be decisive for activity, as flavonoids containing this group were more potent. The number of methoxy groups is also essential for potency, being the most metoxylated compounds the most active [76]. Tiliroside (41), an amphipathic kaempferol glycoside, reduced the concentration of antibiotic needed to inhibit the growth of bacteria. The lipophilicity of the flavone moiety may be a determinant factor for the activity displayed. Tiliroside (41) showed no antibacterial activity against $S$. aureus, but showed a decrease in the MIC of fluoroquinolones up to 16-fold, when at a concentration of $64 \mu \mathrm{g} / \mathrm{ml}$. The reduction in the MIC of biocides, such as ethidium bromide and benzalkonium chloride, was impressive, with the MIC of acriflavine showing a decrease by 128 -fold at the concentration of $64 \mu \mathrm{g} / \mathrm{ml}$ and $32 \mu \mathrm{g} / \mathrm{ml}$. These results suggest that $\mathbf{4 1}$ might additionally act on pumps other than NorA [77].

Kaempferol 3-O- $\alpha$-L-(2, 4-bis- $E$ - $p$-coumaroyl)rhamnoside (42) was also able to potently inhibit in a concentration dependent manner NorA-mediated ethidium bromide efflux in $S$. aureus. This compound also increased the antibiotic effect of norfloxacin against a wild-type and a NorA overexpressing strain of $S$. aureus. However, it has to be considered that 41 and $\mathbf{4 2}$ are flavonoid glycosides and cinnamoyl esters, and that their activity can become compromised with the activity small intestine $\beta$-glucosidases or plasma esterases, even though the activity might possibly lie in the kaempferol moiety, with $\mathbf{4 1}$ and $\mathbf{4 2}$ acting as prodrugs. The authors hypothesized that if the activity of compound $\mathbf{4 2}$ is compromised, this compound could be used as adjuvant of ciprofloxacin for topical infections [78].

Since 42 showed promising results as a NorA EPI, the same group performed a screening of a library of 117 chalcones, due to their structural similarities with the coumaroyl substituents [79]. Ten out of the 117 screened chalcones 
presented medium efflux inhibition at $20 \mu \mathrm{g} / \mathrm{ml}$. The most potent chalcones, such as $\mathbf{4 3}$, bear a dimethylaminoethoxy moiety, which might disrupt the proton gradient, thus eliminating the energy source of NorA. Five of these chalcones possessed a hydroxyl group at the 4'-position, and potentiated the activity of berberine against $S$. aureus and $B$. cereus, suggesting that this substituent is also important for activity. The compound with a methoxy group at position $\mathrm{C}$ 2 has shown to present the best activity. The majority of chalcones tested showed a 4-fold reduction of ciprofloxacin's MIC in $S$. aureus, suggesting the presence of a NorA efflux pump inhibitory activity. Compound $\mathbf{4 3}$ showed 8-fold and 16-fold reduction of the MIC of ciprofloxacin at 6.25 and $12.5 \mu \mathrm{g} / \mathrm{ml}$, respectively. However, it also showed antibacterial activity at $25 \mu \mathrm{g} / \mathrm{ml}$. Some chalcones also presented antiparasitic activity, namely against Leishmania major and Plasmodium yoelii [79].

Another chalcone, 4',6'-dihydroxy-3',5'-dimethyl-2'methoxychalcone (44), showed good activity as a MDR, causing complete growth inhibition at a very low concentration $(3.3 \mu \mathrm{g} / \mathrm{ml})$ when combined with a sub inhibitory concentration of berberine. When tested in combination with berberine, erythromycin, and tetracycline against strains of $S$. aureus, there was an increase in the activity of all tested compounds in the wild-type $S$. aureus, indicating a mode of action likely related to the NorA pump. This compound was also tested against $B$. cereus, potentiating the effects of the tested antibiotics as well, and displaying a favorable effect, particularly in the case of berberine, where a 30 -fold increase in the activity was observed [80].

Pheophorbide A (45) is an intermediate of the natural breakdown of chlorophyll. Concentrations as low as 0.5 $\mu \mathrm{g} / \mathrm{ml}$ of pheophorbide A with sub-inhibitory concentrations of berberine were enough to completely inhibit $S$. aureus growth. When extended to fluoroquinolones, this compound also proved efficient in potentiating the effect of norfloxacin by 4-fold in a wild-type strain, being its action directed towards NorA [81].

Orizabins, oligosaccharides from resin glycosides, were evaluated for their efflux pump inhibitory activity on strains of $S$. aureus. These amphipathic compounds showed synergy with norfloxacin, whilst showing no antibacterial activity by themselves. Orizabin IX (46) completely inhibited the growth of a NorA overexpressing strain at $1 \mu \mathrm{g} / \mathrm{ml}$, while orizabin XIX (47) reversed norfloxacin resistance by 4-fold at $25 \mu \mathrm{g} / \mathrm{ml}$ for the same strain [82]. Murucoidins, which are also resin glycosides, strongly potentiated the action of norfloxacin against a $S$. aureus NorA overexpressing strain by 4 -fold at concentrations ranging from 5 to $25 \mu \mathrm{g} / \mathrm{ml}$. Stoloniferin I potentiated the same activity by 8 -fold at a concentration of $5 \mu \mathrm{g} / \mathrm{ml}$ [83].

A study aiming to find new EPIs from the plant Geranium caespitosum led to the isolation of novel acylated neohesperidosides. It was found that two compounds, compound 48 and 49, were successful at restoring susceptibility to berberine, norfloxacin, and ciprofloxacin at sub inhibitory concentrations, possibly due to NorA inhibition [84].

Capsaicin (50), the major constituent of the fruits of the Capsicum genus, is a known P-gp inhibitor. Studies were performed, in order to evaluate if its inhibitory activity was also applied to MDR pumps in bacteria. In a strain of $S$. aureus overexpressing the NorA efflux pump, and therefore resistant to fluoroquinolones, $\mathbf{5 0}$ proved efficient in increasing the susceptibility to ciprofloxacin, while reducing the emergence of ciprofloxacin-resistant mutants. Molecular modelling studies of the complex capsaicin (50)-NorA, using the glycerol-3-phosphate transporter to predict the structure of NorA, showed that compound $\mathbf{5 0}$ aliphatic chain extended into NorA's hydrophobic cleft. Furthermore, a weak hydrogen bond between Arg-98 and the aryl hydroxyl contributed to the stability of the complex [85].

Studies in the aqueous extract of Artemisia absinthium showed promise in the inhibition of MDR efflux pumps. It was proven that this activity came from caffeoylquinic acids. At a concentration of $20 \mu \mathrm{M}, 4$ '-5'-O-dicaffeoylquinic acid (51) potentiated the activity of berberine by 16-fold and of norfloxacin, by 10 -fold against a wild-type strain of $S$. aureus, while not displaying effects in a norA-deleted strain, confirming this compound's NorA inhibition ability [86]. Olympicin A (52) is an acylphloroglucinol, found in the aerial parts of Hypericum olympicum. This molecule had some efflux impeding ability, proven by the improvement of intracellular accumulation of enoxacin in a NorA overexpressing strain of $S$. aureus, at $50 \mu \mathrm{g} / \mathrm{ml}$ [87].

Riparins are natural alkylamides found in the plant Aniba riparia. Since the extraction of these compounds comes with very low yields, synthetic approaches were developed. As such, the fundamental core of riparins, Rip-A (53), was synthesized and derivatives were prepared, Rip-B (54) to E (55). Rip-E (55) showed good growth inhibitory activity against $S$. aureus, attributed to their lipophilic nature and the presence of hydroxyl groups at the benzamide moiety. Oppositely, Rip-B (54), with two methoxy groups at the phenyl-ethyl moiety did not present antibacterial activity, presenting instead a decrease in the MIC of ciprofloxacin and norfloxacin, similarly to reserpine. Combined with the ability of decreasing ethidium bromide's MIC, the activity of compound 54 was attributed to NorA inhibition, suggesting its potential as an EPI [88].

Extracts of Mirabilis jalapa have shown good results in reversing fluoroquinolone resistance in strains of $S$. aureus overexpressing the NorA efflux pump. One compound in particular, $\quad \mathrm{N}$-trans-feruloyl-4'-O-methyldopamine (56), caused an 8-fold reduction in the MIC of norfloxacin, making it a potential lead in the search for structurally similar synthetic alkylamines [89].

Synthesis of derivatives was undertaken, taking $N$-transferuloyl-4'-O-methyldopamine (56) as a model. The aim was to identify active compounds from natural sources and, as such, couplings between cinnamic acid derivatives and natural-occurring amines were chosen. SAR criteria was taken into account, as it is known that substitution of the aromatic rings, methoxy or hydroxyl substitution, double 
bonds and aromatic ring nature in the amine part influence the activity. The lead compound was $\mathrm{N}$-trans-3,4-Odimethylcaffeoyl dopamine (57) since it showed the same activity as the natural compound. It was found that, for the cinnamic moiety, a hydroxyl substitution on the aromatic ring appears to be better than a methoxy group or unsubstituted derivatives and the double bond is essential for activity. As for the amine part, trisubstitution on the aromatic ring increases antibacterial activity, at the cost of decreased efflux pump inhibition. Methoxy substitution yields compounds with better results than hydroxyl substitution, which was better than no substitution and tryptamine combinations showed the best results (Figure 7). Among the compounds tested, compound $\mathbf{5 8}$ showed potentiation of norfloxacin comparable to that of reserpine (30). At a concentration of $30 \mu \mathrm{M}, \mathbf{5 8}$ showed good results for the inhibition of ethidium bromide efflux. For these compounds, it was shown that the activity reaches its highpoint when the phenyl ring is substituted with two hydroxyls [89].

Grapefruit oil fractionation led to the isolation of three compounds, two coumarin derivatives and one bergamottin derivative. The bergamottin epoxide (59) showed a reduction of ethidium bromide's MIC by 6-fold on different MRSA strains, and a 20-fold reduction in norfloxacin's MIC. This reduction was also achieved when the coumarin epoxide (60) was present, and suggests an activity towards NorA efflux pumps [90].

Coumarins isolated from Mesua ferrea were also studied for their efflux pump inhibitor activity against clinical strains and NorA overexpressing strains of $S$. aureus. Seven coumarins were tested, and showed good results in inhibiting ethidium bromide efflux. Two compounds, 61 and 62 , showed potential EPI activity against the NorA overexpressing strain and a MRSA strain, using norfloxacin as a substrate, which makes them desirable compounds for the synthesis of derivatives [91].

Screening of natural products with P-gp inhibitory effects $(92,93)$ led to the identification of two compounds with NorA inhibitory activity, osthol (63) and curcumin (64) [36, 92-93]. Curcumin (64), derived from the rhizomes of Curcuma longa, has additionally already shown activity as an anti-inflammatory, antioxidant, antiviral, and anticancer agent [36, 92-93]. These compounds were effective in reducing the MIC of ciprofloxacin by 4- and 8-fold, respectively [94].

A penta-substituted pyridine, 2,6-dimethyl-4-phenylpyridine-3,5-dicarboxilic acid diethyl ester (65), was isolated from the rhizomes of Jatropha elliptica. This compound, when in association with ciprofloxacin, showed a strong effect against NorA efflux pumps in $S$. aureus [95].

A methanol extract of the plant Dalea spinosa yielded the isolation of six compounds, whose EPI potential was analyzed against wild-type $S$. aureus and mutant strains. It was shown that spinosan A (66), at a concentration of 48 $\mu \mathrm{g} / \mathrm{ml}$, and its acetate 67 , at a concentration $42 \mu \mathrm{g} / \mathrm{ml}$, were able to decrease berberine's MIC by 8 - and 62-fold, respectively, against the wild-type. Other compounds were also able to reduce berberine's MIC, but to a lesser extent.
Compound 67 also managed to decrease berberine's MIC in the NorA overexpressing strain, suggesting inhibitory activity in this efflux pump [96].

\subsubsection{Synthetic Compounds}

The INF series (compounds 68-72) resulted from an initial screening of 9600 structurally diverse compounds, in order to find NorA inhibitors for B. subtilis, not expressing Bmr. The NorA substrate chosen was ethidium bromide [67]. Since this screening did not focus on a specific chemical group, the most active compounds were divided into several groups. Several indole derivatives were active, which did not come as a surprise, since reserpine (30) itself has an indole moiety. Another large group was the trichloromethylaminal containing compounds, which were not further explored as their likelihood to be toxic was high. Biphenyl urea derivatives were also active, and other compounds, with no obvious similarities, showed activity as well. Five compounds were selected for further tests, INF 55 (68), INF 240 (69), INF 271 (70), INF 277 (71) and INF 392 (72). These compounds were active at concentrations of $5 \mu \mathrm{g} / \mathrm{ml}$ or less, and included the most potent compound, 72, the most potent indole, 68, and the most potent biphenyl urea, $\mathbf{7 1}$. Derivative $\mathbf{7 2}$ was able to reduce resistance to ethidium bromide and ciprofloxacin by 8 -fold at a concentration of 0.4 $\mu \mathrm{g} / \mathrm{ml}$. All five inhibitors were more potent than reserpine (30) at promoting the activity of ciprofloxacin in $S$. aureus and, by being structurally different, it was hypothesized that a potent, nontoxic lead would be identified. These compounds also proved effective in inhibiting the $\mathrm{Bmr}$ multidrug transporter in B. subtilis and two compounds, 68 and 71, also enhance fluoroquinolone activity in Streptococcus pneumoniae [67].

The results obtained with INF 55 (68) in $S$. aureus as an inhibitor of the NorA efflux pump led to an increased interest in this kind of scaffold. SAR studies regarding the C5 proved that substitution in this carbon is crucial for activity. Substitution with a nitrile group leads to retention of potency, and carbonyl based electron-withdrawing groups at C-5 would result in molecules with no activity [97]. Three dimensional-quantitative structure-activity relationship (3DQSAR) studies predicted that 2-aryl indole derivatives would be NorA inhibitors. Functionalized INF 55 (68) showed a decreased MIC for berberine, a MDR transporter substrate. Berberine is an amphipathic cation alkaloid. From the series of 2-aryl-5-nitroindoles synthesized by Samosorn et al. (2009), the alcohol $\mathbf{7 3}$ was the most effective in inhibiting berberine efflux, as well as other antibiotics, such as ciprofloxacin, in S. aureus [98]. Later, the same group developed a strategy that consisted in a molecule presenting dual action: an antibiotic and a MDR inhibitor. Therefore, they combined berberine, a hydrophobic cation that is a MDR substrate, with INF 55 (68), a MFS inhibitor. These efforts yielded compound 74 [17-18, 99]. In terms of antibacterial activity in $S$. aureus, the hybrid was about 100 times more active than berberine alone, and the difference was even higher when tested against a NorA overexpressing 
strain of $S$. aureus. Enterococcus faecalis also presented resistance to berberine, but was susceptible to 74. [99] Synthesis of sulfur derivatives was achieved based on the premise that the activity of $\mathbf{6 8}$ is less dependent on the nitro group than the 2-arylindole. Benzothiophene, thiophene, and benzofuran derivatives, such as compounds $\mathbf{7 5}, \mathbf{7 6}$, and $\mathbf{7 7}$, respectively, were able to synergize with ciprofloxacin and ethidium bromide, increasing their intracellular concentration and leading to the conclusion that these compounds inhibit NorA in S. aureus. [100]

Taking into account the fact that the indole $\mathrm{NH}$ is not essential for efflux pump inhibition, Samosorn et al. (2009) simplified the indole moiety of berberine-based hybrids, along with the effect of a methylene ether linking group. Their results showed that a hybrid molecule with oxygen in the linkage chain had stronger antibacterial and MDR pump inhibitory activity than the original 74 [101]. On the other hand, removal of the indole moiety produced compounds with lower dual activity, and this structural simplification led to compounds with low antibacterial activity, but with retained NorA pump inhibitory activity. These data suggest that while the indole moiety is not necessary for NorA pump inhibition, it is required for the antibacterial activity of hybrid compounds [101].

A recent study identified a novel class of NorA EPIs with a polysubstituted indole moiety. Twenty molecules were highly active in inhibiting ethidium bromide efflux in $S$. aureus. However, the best results were obtained with compounds 78 and 79, which were derivatives that presented three and four carbon atom chains, respectively. Compound 78 presented the best half maximal inhibitory concentration $\left(\mathrm{IC}_{50}\right)$, with a concentration of $1.8 \mu \mathrm{M}$. Compound 79 exhibited similar $\mathrm{IC}_{50}(2 \mu \mathrm{M})$ and ethidium bromide inhibition as 78. Compounds with shorter or longer carbon chains did not present such good results, leading to the conclusion that three or four carbons make up the right distance between the indole scaffold and the basic center, thus presenting the best inhibitory effect. When tested for their synergy with ciprofloxacin, both $\mathbf{7 8}$ and 79 were able to restore the antibiotic's activity [102]. It was found that the substitution of the $\mathrm{C}-5$ in the indole results in a potent EPI, particularly if there is a propoxyl chain carrying terminal cyclic amino groups, which appears to be crucial for NorA inhibition at low concentrations. The presence of the $N$ benzyl moiety preserves inhibition and contributes to the modulation of the biological effects and ADME properties, depending on its substituents [103].

Another successful example of a hybrid antibacterial/EPI is the fluoroquinolone scaffold accommodating a bis-aryl urea EPI moiety at the C-7 position. This kind of compounds was successful in inhibiting NorA pumps in $S$. aureus. Compound 80, which incorporates a bis-aryl urea motif into the ofloxacin core, proved to be a potent inhibitor of MFS efflux pump systems, achieving $84 \%$ inhibition rate at 10 $\mu \mathrm{M}$. Moreover, these compounds also successfully inhibit MepA pump systems from the MATE family [17-18, 104]. Fluoroquinolones containing a thiopyranopyridine moiety at the $\mathrm{C}-7$ position were synthesized and their activity was tested against strains of $S$. aureus. However, these derivatives did not seem to be NorA substrates. Further investigations into thiopyranopyridinylquinolone esters were carried out. Some of the synthesized compounds displayed better inhibitory activity than reserpine (30), namely quinolone esters $\mathbf{8 1}, \mathbf{8 2}$ and $\mathbf{8 3}$, and showed significant MIC reduction of ciprofloxacin. Compounds $\mathbf{8 2}$ and $\mathbf{8 3}$ also showed a 32-fold MIC reduction of ciprofloxacin for strains of $S$. aureus overexpressing MepA pumps from the MATE family $[18,105]$. In terms of established SAR, these compounds definitely do not need the fluorine atom at the $\mathrm{C}$ 6 position for the antibacterial activity of thiopyridinylquinolones. A methyl at the C-8 position yields a compound with enhanced activity against Gram-positive bacteria for 6-amino derivatives. The thiopyranopyridine moiety at the C-7 position is crucial for NorA and MepA inhibition (Figure 8) [105].

Derivatives of 2-(4-propoxyphenyl)quinolone were synthesized based on the premise that its large hydrophobic area and ability to establish an electrostatic interaction would make them suitable inhibitors of NorA. These derivatives also mimic the quinolone antibacterial core and possess a versatile scaffold, making it prone to be very simply chemically modified. These classes of compounds were obtained by performing modifications in the 2-phenyl- $4 \mathrm{H}$ chromen-4-one moiety, which is a common feature of flavone and flavolignane EPIs [106].

Studies showed that the $O$-substituted 2-phenyl-4hydroxyquinoline derivatives displayed the best activity as NorA inhibitors. Compounds 84 and 85 showed synergistic activity against a strain of $S$. aureus overexpressing NorA efflux pumps comparable to reserpine (30) and, comparing the results obtained with a mutant strain, absent of NorA, it was found that these compounds are able to completely restore the antibacterial activity of ciprofloxacin in the resistant strain [18, 106]. Preliminary SAR studies for this new class of EPIs showed that the best activity is when the quinolone core is replaced with the 2-phenyl-4hydroxyquinoline group with an alkylation in the C-4 hydroxyl group. 2-Ethylamino chains inserted at the $\mathrm{N}-1$ position of the quinolone nucleus or at the C-4 hydroxyl of the quinoline moiety provided compounds with better activity than those carrying the same chain at the C-2 of the phenyl ring. The best substituent for the C-2 phenyl ring appears to be the C-4' propoxy group (Figure 9) [106].

Taking these results into account, efforts have been placed to obtain more potent NorA inhibitors, introducing $O$-alkyl or different $O$-alkylamino chains at the $\mathrm{C}-4$ position. The strategy was to determine the essential $3 \mathrm{D}$ structural requirements for the inhibition of NorA, based on the NorA EPIs described in literature through the use of computational methods, since the 3D structure of this pump was not elucidated yet. The results led to the building of a possible pharmacophore, which was used to design novel compounds, with different substituents at the C-4 position, which were synthesized and biologically evaluated [107]. The compounds were assayed for their ability to reduce the MIC of ciprofloxacin in $S$. aureus strains, and all compounds 
displayed this decrease for the strain overexpressing NorA pumps. One compound in particular, 86, showed a 16-fold reduction in this strain for ciprofloxacin's MIC. SAR studies for this emerging class show that the best activities come from the compounds bearing the 2-ethylaminoalkyl chains linked to the C-4 hydroxyl group, such as compound $\mathbf{8 7}$. The inclusion of an aliphatic ring including the side chain nitrogen atom led to retention of activity, whereas inclusion in an aromatic ring led to loss of activity, where the nitrogen nucleophilicity was reduced. The mono-substituted compounds, like $\mathbf{8 8}$, with a benzyl group were well tolerated, and showed higher ethidium bromide inhibitory activity than compounds with two benzyl groups as substituents (Figure 9) [107].

3-(Dihydronaphtyl)-propenoic acid amines were evaluated for their EPI activity. Five compounds were synthesized, and two of them showed good results in inhibiting ciprofloxacin efflux in strains of wild-type and NorA overexpressing $S$. aureus. Further studies were performed, given the low EPI activity of this kind of compounds. Fifteen different compounds were synthesized, taking $\alpha$-tetralone (12) as starting material. Out of these, four compounds showed significant MIC reductions for ciprofloxacin for the NorA overexpressing strain. One compound, 89, even displayed a 16-fold reduction, while other remained in the range of 4- to 8 -fold. These studies allowed SAR to be established. Addition of methoxy or allyloxy substituents at the 6,7position of the 3,4-dihydronaphtalene leads to lower or no activity, whereas no substitution originated better EPIs. Saturation of the double bonds lowered the potentiating activity (about 2-fold), while unsaturated derivatives made much more potent compounds [108].

Boronic species have been described for a variety of activities, namely antimicrobial and antineoplasic. Organoboron compounds, oxazaborolidines, boronic esters, and boronic acids are of particular interest, within this class. Fontaine et al. (2014) developed studies on these molecules, starting from a screening of a library of approximately 150 compounds, out of which 24 were hit compounds (exemplified with compounds 90 and 91), and were able to restore the activity of ciprofloxacin by 4-fold at concentrations of 0.5 to $8 \mu \mathrm{g} / \mathrm{ml}$ against the NorA overexpressing strain of $S$. aureus [109]. Preliminary SAR studies demonstrated the need of the boron atom for activity. Most pyridine-3-boronic acids showed best potentiating activity (e.g., compound 90), whereas pyridine-4-boronic derivatives showed no activity, with exceptions, including a fluorinated ester. The shift of the boronic moiety from C-3 to $\mathrm{C}-4$ led to a loss of activity and the trigonal boronic acid function gave better results that the sodium boronate salts and the potassium trifluoroborate. Concerning the pyridine3 -boronic acids, the most promising compounds appear to be the 6- and the 5,6-disubstituted ones, being the latter compounds less potent. Cyclisation at the C-5 and C-6 positions was well tolerated, as was the introduction of a methyl at the C-5 position. For the benzene analogues, which displayed good activity, cyclisation at the C-3 or C-4 was also well tolerated, as was the introduction of a methyl. A substituent at the para position to the boronic moiety proved to be very important. Two compounds were particularly efficient in inhibiting NorA, and did not display intrinsic antibacterial activity and cytotoxicity: the 6benzyloxypridine-3-boronic acid 90, which displayed the highest activity, potentiating ciprofloxacin by 4 -fold at a concentration of $16 \mu \mathrm{g} / \mathrm{ml}$, and the 4-benzylxybenzene boronic acid 91 [109].

Compound 90, being the most promising compound, was considered a hit, and its structure was used as a model for the synthesis of new derivatives. Therefore, the 6-benzyloxy group was substituted with (aryl)alkoxy chains with variable lengths (compounds $\mathbf{9 2}$ and $\mathbf{9 3}$ as examples), in an attempt to explore the putative hydrophobic binding site of NorA, and substituents were added to the phenyl ring. The ether linkage between the pyridine ring and the 6-benzyloxy group was substituted as well. The first substitution made was the replacement of the 6-benzyloxy group with alkoxy chains of diverse lengths, as to explore the size of the hydrophobic pocket of NorA. The potency of the compounds increased with the increase of the size of the side chain, although the compound with a 11-carbon side chain displayed no activity whatsoever. A new series of compounds with a phenyl ring at the end of the side chain were synthesized, with the aim of enhancing hydrophobicity, and the results showed these compounds were more efficient. It was concluded that the compound with the best performance possessed a side chain with seven carbon atoms and a phenyl group: compounds 92, 6-(3-phenylpropoxy)pyridine-3-boronic acid, and 93, 6-(4phenylbutoxy)pyridine-3-boronic acid, showed the highest activity in the potentiation of ciprofloxacin (4-fold). The introduction of substituents in the phenyl ring of compound 90 led to unsatisfactory results, with the best compound displaying moderate antibacterial activity. A methyl group in the C-5 position did not show influence on the activity, and the modification of the ether linkage led to varied effects: introduction of a sulfanyl, thus conserving the H-bonding acceptor capacity, led to similar potentiating activity, whereas the introduction of an amine linkage, an H-bond donor, led to a decrease in the activity. Finally, the shift of the benzyloxy group from the C-6 to the C-5 resulted in no activity [110].

Compounds AE-848/42434549 (94) and AN-465/42885978 (95) were hits in a virtual screening of novel NorA efflux pump inhibitors in $S$. aureus. The structures deemed as active in silico were later synthesized and evaluated on their ability to prevent ethidium bromide efflux, as well as ciprofloxacin potentiation. Compound 94 and 95 were as potent as reserpine (30) for ethidium bromide efflux and more potent at synergizing with ciprofloxacin. These hit compounds 94 and 95 paved way for the synthesis of new analogues, which allowed SAR studies to be made, in order to assess which parts of their structure were determinant for the inhibition of NorA. For compound 94, it was shown that limited modifications to the sulfone and amide group do not cause significant changes in activity. On the other hand, modifications to the imidazole ring result in a reduction of activity. Replacement of the 1-methylpyrrole ring with a 
furan also shows a decrease in the activity of the compound. As for compound 95, the $O$-benzyl group is essential and the introduction of a benzyl in the secondary nitrogen leads to a complete loss of activity. Furthermore, the methyl group of the 2-(1-phenyl-1-propanol) moiety can be replaced with a 2(1-phenyl-1-ethanol) portion without a decrease in the activity [111].

\subsubsection{Existing Drugs}

Several drugs already available for the treatment of various diseases have shown potential to be used as MFS EPIs. Reserpine (30), mentioned before, is one of them. Other drugs include verapamil, omeprazole, paroxetine and chlorpromazine. Even though bacterial MFS transporters and mammalian $\mathrm{ABC}$ efflux systems, as P-gp, lack structural homology, they share similar substrate profiles, which means that mammalian MDR inhibitors can also present bacterial efflux inhibition. However, the concentrations needed for efflux pump inhibition are too high, which means that toxic effects can arise if these compounds are used for this purpose [18], [112]. In fact, the addition of verapamil to chemotherapy was accompanied with cardiac arrhythmia and hypotension [113]. This leads to the requirement of developing analogues of these existing drugs maintaining the desired EPI activity at concentrations that do not display toxicity [112].

The antiarrhythmic verapamil, a known P-gp inhibitor, has shown moderate NorA inhibitory activity in both $B$. subtilis and $S$. aureus, enhancing fluoroquinolone activity [114-115]. However, the best results were obtained with proton pump inhibitors omeprazole (96) and lansoprazole. Therefore, a series of pyrrolo[1,2- $a$ ]quinoxaline derivatives mimicking the omeprazole structure (96) were designed, with the aim of evaluating their EPI activity against NorA. All the eleven derivatives showed a reduction in norfloxacin's MIC, being compound 97 the most active, reducing the MIC by 16-fold at $128 \mu \mathrm{g} / \mathrm{ml}$. These compounds did not show disturbance of the electrical potential and the transmembrane $\mathrm{pH}$, excluding the alteration of the proton-dependent pump as the mechanism of action. Instead, it is thought that these compounds interact directly with NorA. It was shown that the benzimidazole moiety is critical for the activity of these compounds (Figure 10), and replacing it with an imidazole, a pyridine or a pyrrolo[1,2-a]quinoxaline ring resulted in loss of potency. Furthermore, if there is a methoxysubstituent in the benzimidazole ring, potency increases. Introduction of chlorine in the C-7 of the pyrroloquinoxaline heterocycle provided an efficient restoration of norfloxacin's bactericidal activity. Finally, the benzimidazolyl group was also important for activity [112].

This was not the first time that pyrrolo[1,2-a]quinoxaline derivatives were described as EPIs. In fact, a previous study by the same group produced 12 new 4-[116]pyrrolo[1,2$a]$ quinoxalines and one pyrrolo[1,2- $\alpha]$ thieno[3,2-e]pyrazine compound. Two compounds, 98 and 99, were more active than reserpine (30), diminishing norfloxacin's MIC by 16fold in a NorA overexpressing strain of $S$. aureus. This study showed that 4-[116]pyrrolo[1,2-a]quinoxalines and their 7 methoxy analogues were more active, in contrast with the chlorine- and 8-phenyl-substituted compounds, that presented the lowest effects. Replacement of the $\mathrm{N}, \mathrm{N}$ diethylamino group by pyrrolidine, its isomer with restricted conformation, led to enhanced EPI activity, whereas replacing it with a piperidine ring led to more flexible and, consequently, less active compounds. The pyrrolothienopyrazine 99 moiety yielded the best results, suggesting that sulfur, an electron-rich atom, potentiates the EPI activity [116].

GG918 (19) was discovered in a screening program aimed to identify inhibitors of mammalian P-gp, and was able to increase the concentrations of paclitaxel. This compound did not present antibacterial activity against the strains of $S$. aureus tested. However, the co-administration with norfloxacin resulted in a 4-fold reduction in the MIC of the NorA overexpressing strain [52].

Two mammalian MDR inhibitors, biricodar (100) and timcodar (101), confer increased drug sensitivity to cells expressing both the P-gp and multidrug resistance protein (MRP)-1 efflux systems. They also show potentiation of multiple antibiotics in Gram-positive bacteria. In fact, both compounds were able to potentiate the activity of ethidium bromide against $S$. aureus, E. faecalis, and $S$. pneumoniae. For the specific case of $S$. aureus, both compounds were able to partially reverse fluoroquinolone resistance. The fact that the MIC of ethidium bromide was non-saturable for the three bacteria tested suggests that $\mathbf{1 0 0}$ targets multiple efflux pumps [117].

A series of polysubstituted pyrroles was screened for their Pgp inhibition activity, which made way for the identification of a lead, and the compounds were further investigated for their bacterial efflux pump inhibition activity. A simplified pyrrole alkaloid, 102, displayed dual activity as both a P-gp and a NorA inhibitor against $S$. aureus. This compound led to 4-fold improvement in the MIC of ethidium bromide in the NorA overexpressing strain. Compound $\mathbf{1 0 2}$ was also able to reduce ciprofloxacin's MIC by 8 -fold when at $50 \mu \mathrm{M}$ [118].

Tariquidar (103), a third generation MDR modulator, with activity in inhibiting P-gp and BCRP efflux systems, has shown promise in inhibiting efflux pumps in $S$. aureus. In fact, this compound showed an increase of the isotopic form of ciprofloxacin in all investigated strains, displaying best results in the strain that overexpresses the NorA pump. [119] The class of phenylpiperidine selective serotonin reuptake inhibitors (PSSRI), which includes the antidepressant paroxetine (104), showed efflux-related potential against $S$. aureus, particularly that conferred by NorA. In fact, paroxetine and its isomer, $\mathbf{1 0 5}$, showed potency with respect to inhibition of this efflux pump against ethidium bromide. The isomer $\mathbf{1 0 5}$ also interfered with the accumulation of norfloxacin by NorA, reducing by 4 - to 8-fold its MIC. [120] Femoxetine (106) is a paroxetine derivative, with activity as an EPI. [120] Studies were performed as to determine which moieties in paroxetine- and femoxetine-like PSSRI were detrimental for efflux pump inhibition in $S$. aureus, and 
conclusions from SAR are highlighted in Figure 11. Synthesis of derivatives showed that the fluorine atom on the phenylpiperidine moiety is not associated with the activity. The $N$-substitution of the piperidine ring yields different kinds of activity, suggesting that the NH group forms a positive binding contact with the target pump, which does not happen if there is a $N$-substitution (compound 106). The phenyl ether substituents also play an important role as determining the EPI activity. [121]

Also for this kind of compounds, a study was performed, in order to conclude whether the 4-phenyl ring is required for EPI activity and the effects of changes in the two-atom aryloxymethyl linker, replacing it with a thioether, amine, and alkene linker portions. Compounds 107 and 108 displayed the lowest MIC against $S$. aureus. Synthesis of multiple derivatives proved that the 4-phenyl moiety is not vital for EPI activity and that a variety of two-atom linker groups for the 3-aryl piperidine moiety can maintain EPI activity [122].

The ciclooxigenase-2 (COX-2) inhibitor celecoxib (109) has also received attention for its MDR inhibitory activity. There had been a link between COX-2 and $m d r l$ gene expression, which has consequences in cancer chemo resistance, and the use of celecoxib (109) reversed drug resistance. If this is true for mammalian efflux pumps, for bacteria there is no evidence of a COX-2-like gene, suggesting that celecoxib acts differently in inhibiting MDR in bacteria. A study was carried out in order to prove this hypothesis, which demonstrated that this drug helped in increasing sensitivity to antibiotics in S. aureus and Mycobacterium smegmatis. These effects probably occur due to the blockage of MDR transporters involved in the efflux of antibiotics, such as ampicillin, ciprofloxacin, kanamycin, and chloramphenicol. The observation of the accumulation of ethidium bromide intracellularly leads to the conclusion that this compound acts as a NorA inhibitor [123].

As celecoxib (109) demonstrated such promising activity, analogues were tested, which bore low or none antiinflammatory activity and possessed the 1,4dihydropyrazolo[4,3-c]-benzothiazine-5,5-dioxide nucleus. Ethidium bromide efflux inhibition was tested first in silico, and the 17 molecules that fit the chemical space requirements were then reduced to six, based on the activity prediction. These compounds were then screened for their in vitro NorA inhibitory activity, using an overexpressing strain of $S$. aureus. One derivative in particular, compound 110, showed the best activity concerning ethidium bromide efflux $(76.9 \%)$ which is better than celecoxib (109) $(64.5 \%)$. When in combination with ciprofloxacin, $\mathbf{1 1 0}$ displayed results comparable to reserpine (30) and better than paroxetine (104), which makes it a lead compound for the development of inhibitors of this kind [124].

Phenothiazine and thioxanthene derivatives are currently used as neuroleptic and antiemetic agents, and have been described as modest, but broad, antibacterial agents. Although their antibacterial activity is not enough to be clinically relevant, they have shown synergy with standard antibiotic agents, and have also been associated with the inhibition of eukaryotic MDR efflux pumps, such as P-gp. The mechanism through which these compounds exert their antimicrobial potentiation is not yet fully understood. However, it is thought that it might have to do with the inhibition of efflux pumps [125]. A study focusing on the activity of several compounds of these families against $S$. aureus was carried out, with the main aim of showing their potential in inhibiting NorA. The compounds tested, chlorpromazine, fluphenazine, thioridazine, prochlorperazine (111), cis(Z)-flupentixol, and $\operatorname{trans}(E)$-flupentixol (112), displayed intrinsic antimicrobial activity and good results when ethidium bromide efflux was assayed against a NorA overexpressing strains of $S$. aureus. Additionally, phenothiazine 111 and thioxanthene $\mathbf{1 1 2}$ were also able to reduce the proton motor force of $S$. aureus, by reducing the transmembrane potential [125].

Since the phenothiazine moiety proved itself to work as a template for the synthesis of new MDR EPIs, it was chosen to be the scaffold for derivatives with improved activity. The logic for these studies lied in the elimination of the structural features that were responsible for neuroleptic activity. As such, drastic modifications were made, such as the elimination of one ring of the tricyclic benzothiazine backbone and of the chain linked to the N-10 atom, a tertiary amine whose protonation is crucial for the interaction with the dopaminergic receptor, and the addition of a substituted phenyl ring at the C-3 position, to guarantee better lipophilicity. Therefore, a prototype of new 3-phenyl-2H1,4-benzothiazines was developed. Preliminary screening on the derivatives using a wild-type $S$. aureus strain, both with and without ciprofloxacin, showed variable intrinsic and synergistic activity. Two compounds, 113 and 114, displayed the best activity, while displaying no and weak antibacterial activity, respectively. These compounds were able to inhibit NorA efflux of ciprofloxacin, being suggested that 114 was even able to completely inhibit this pump [126].

Thioridazine (115), a phenothiazine, was also tested for its antibiotic potentiation, either in racemate or in its enantiomeric forms. All the forms were able to reduce the MIC of oxacillin in $S$. aureus and erythromycin in Streptococcus pyogenes, being the L-enantiomer more effective in the last case [127-128].

\section{RND}

RND pumps are found mostly in Gram-negative bacteria, being the most responsible pumps for MDR in this kind of organisms. They present a wide variety of lipophilic and amphiphilic substrates, which include several classes of antibiotics, antiseptics, dyes, and detergents. RND transporters present a unique tripartite complex, constituted by a minimum of twelve transmembrane segments: the transmembrane pump, the outer membrane channel, and the periplasmic adaptor protein, which puts the other two components in contact. This structural organization makes the extrusion of substrates directly into the external medium possible [32, 38]. Even though it has always been thought that RND transporters were exclusive to Gram-negative 
bacteria, it has recently been identified and characterized FarE in S. aureus, homolog to the AcrB protein in E. coli [129-130].

Within this family, the most studied system is the AcrABTolC, from E. coli. This efflux pump is composed by three essential parts: a resistance-nodulation-division transporter, AcrB; a membrane fusion protein, AcrA; and a multifunctional outer membrane channel, TolC [7, 131-133]. It transports a wide variety of toxic compounds from the intracellular space directly into the medium, evading the periplasm, and using a proton gradient as the energy source [132-133]. Pseudomonas aeruginosa also possesses an efflux system of the RND family, the MexAB-OprM efflux pump, which confers resistance to a wide variety of antibiotics. This was the first and best characterized efflux system in bacteria. It was found that AcrB (Figure 12, PDB 4ZLJ) is homolog to MexB (Figure 12, PDB 2V50), being their structure very similar [7, 134].

Figure 13 comprises all the RND pump inhibitors that will be discussed in the following section.

\subsection{RND inhibitors}

\subsubsection{Natural Products and Derivatives}

One study revealed that the essential oil of Helichrysum italicum can reduce the resistance to chloramphenicol in $E$. aerogenes, $P$. aeruginosa, and A. baumanii. These data suggested that one or more EPIs are present within the essential oil since it was shown that geraniol (116) produced significant restoration of sensitivity to chloramphenicol by 16-fold, and rendered a total sensitivity of the organism when combined with of phenyl-arginine $\beta$-naphtylamide $(\mathrm{PA} \beta \mathrm{N})$, which will be discussed further ahead [135].

A study published by Aparna et al. (2014) had the aim of identifying natural compounds from plants that present efficacy in potentiating effects of antibiotics in $P$. aeruginosa overexpressing MexAB-OprM and E. coli overexpressing AcrAB-TolC through in silico virtual screening and pharmacophore approaches [136]. The compounds with the best in silico results were then tested for their EPI activity. The two compounds that showed the best activity in potentiating the activity of carbenicillin and levofloxacin in $P$. aeruginosa and $E$. coli were lanatoside $\mathrm{C}$ (117) and daidzein (118). Lanatoside $C$ (117) is cardiac glycoside that inhibits the $\mathrm{Na}^{+}-\mathrm{K}^{+}$-ATPase, which can also be the explanation for its EPI activity against MexB and AcrB. Daidzein (118) is an isoflavone that has previously showed slight EPI activity in Mycobacterium smegmatis and as a modulator of P-glycoprotein, in human cervical carcinoma KB-V1 cells [136].

An in silico study by Ohene-Agyei et al. (2014) identified five phytochemicals that could be docked similarly to PA $\beta N$ in the binding pocket of AcrB. Of these five, three of them were able to decrease resistance to antibiotics by inhibiting the AcrAB-TolC system: plumbagin (119) increased sensitivity to erythromycin, chloramphenicol, and tetraphenylphosphonium; nordihydroguaretic acid (NDGA)
(120) potentiated the activity of erythromycin, chloramphenicol, tetraphenylphosphonium, novobiocin, and tetracycline; and shikonin (121) decreased resistance to tetraphenylphosphonium [137].

A study proved the usefulness of curcumin (64) as a RND EPI at a concentration of $50 \mu \mathrm{g} / \mathrm{ml}$, increasing the sensitivity of carbenicillin, ceftazidime, and meropenem in $P$. aeruginosa, in decreasing order. It also managed to circumvent the resistance to gentamicin and ciprofloxacin in resistant isolates, which $\mathrm{PA} \beta \mathrm{N}$ failed, leading to the assumption that curcumin inhibits efflux pumps differently from PA $\beta \mathrm{N}$ [138].

A screening of 85000 microbial fermentation extracts derived from 3600 strains of actinomycetes and 3500 strains of fungi was made, with the aim of discovering EPIs that increased sensitivity of levofloxacin in $P$. aeruginosa overexpressing the MexAB-OprM or the MexEF-OprN pump. Two compounds, EA-371 $\alpha$ (122) and EA-371- $\delta$ (123), produced by a strain of Streptomyces sp., demonstrated inhibitory activity against MexAB-OprM [139].

\subsubsection{Synthetic Compounds}

The first compound with potent inhibition of RND efflux pumps was PA $\beta N$ (124), a dipeptide amine. This dipeptide was identified as a hit compound from the screening of 200 000 samples of small molecules that potentiate the activity of antibacterial levofloxacin against strains of $P$. aeruginosa that overexpressed MexAB, MexCD and MexEF pumps [28, 140]. Compound $\mathbf{1 2 4}$ is a peptidomimetic, developed for using in adjunctive therapy. This compound is a substrate for RND pumps, acting as a competitive inhibitor of multidrug efflux systems, such as MexB, MexD, and MexF, binding to the substrate pocket, impeding antibiotic binding and/or extrusion. Particularly, this EPI binds in a location close to the antibiotic binding site, generating steric hindrance, making it more difficult for the antibiotic to bind. In other words, less quantity of antibiotic is exported, making its intracellular levels higher, thus restoring antibiotic sensitivity [11, 25, 27-28, 141-143]. However, 124 can affect the integrity of the membrane when used in high concentrations, making it prone to the emergency of resistant profiles, namely the modification of the lipopolysaccharide structure, leading to changes in drug penetration [24].

PA $\beta N$ (124) has been validated against the AcrAB-TolC in Klebsiella pneumonia, E. coli, Salmonella enterica serovar Typhimurium and Enterobacter aerogenes, and in multiple homologous systems including Acinetobacter baumanii, Campylobacter jejuni, and Campylobacter coli [11, 142]. Its potency has been demonstrated against the norfloxacin resistance conferred by the Mex efflux system of Pseudomonas aeruginosa, the AcrAB efflux systems of the Enterobacteriaceae, and the erythromycin efflux system of $C$. jejuni [19].

Studies of SAR demonstrated that the basic middle amino acid, arginine, was detrimental for activity, although a substitution with L-lysine showed the same potency as the 
lead, and the introduction of L-ornithine, originating compound L-Phe-L-Orn- $\beta$-Na, showed a 2-fold increased potency. As the ornithine moiety provided a simpler synthesis, it became the standard middle residue in successive compounds [25, 142, 144]. However, these compounds were not stable upon incubation with human serum, due to the occurrence of cleavage of peptide linkage between both amino acids, which is explained by the fact that natural (L) amino acids are contained within its structure. In order to overcome this problem, methylation of the NH that links both amino acids was achieved, maintaining potency and being this derivative able to resist serum proteases. Serum stability was also increased by replacing the L-amino acid with D-amino acid [25, 142]. In the same study, the authors also proved that the amino acid in the aal position (Figure 13, 124) should be appropriately substituted, and the replacement of phenylalanine with a homophenylalanine led to improved potency. The problem with this compound was the fact that the ornithine moiety could easily form a lactam, rendering the compound inactive. This was overcome by switching the L-ornithine with the Lphenylalanine, which originated L-Orn-L-hPhe- $\beta-\mathrm{Na}$, which originated a compound just as potent, but with no propensity to form a lactam. As for the $\beta$-aminonaphtalene moiety, it was proven that replacing it with 3 -aminoquinoline led to reduced cytotoxicity and intrinsic antibacterial activity, even though its potency was slightly reduced [25, 142]. These efforts originated the compound L-Orn-L-hPhe-3-NHQ (125). In conclusion, the analogues synthesized by Renau et al. (1999) did not show improvements in potency when compared to PA $\beta \mathrm{N}$ (124), but showed increased stability [142].

The same group developed further studies with this type of molecules and demonstrated that conformational restriction of the amino group of ornithine showed less toxicity, while maintaining potency. Such compounds were attained by adding a proline substituent, thus incorporating the two basic residues necessary for activity, which resulted in the successful synthesis of constrained derivatives, more potent and less toxic than 125. The most interesting derivative, compound 126, was as potent as $\mathbf{1 2 5}$, but more than 4-fold less toxic. This compound also retained the activity against RND efflux pumps MexAB-OprM, MexCD-OprJ and MexEF-OprN. Although this compound showed similar protein binding in rats as 125, its pharmacokinetic parameters were improved, and its efficacy in combination with levofloxacin was demonstrated in an in vivo model [145].

In a latter study developed by the same group, several nonpeptide analogues were synthesized, and it was hypothesized that the peptide backbone is not essential for the activity of this type of compounds. The results suggested that the inhibition of pumps was possible due to the di-cationic nature of the compound, the appropriate lipophilicity and a disposition similar to that of compound 125 [146].

MBX2319 (127) is a pyranopyrimidine EPI inhibitor of AcrAB-TolC efflux pump in E.coli and other Enterobacteriaceae [141]. Compound $\mathbf{1 2 7}$ did not show any antibacterial activity, which is a positive feature for EPIs. It also potentiated the antibacterial activity of fluoroquinolones, such as ciprofloxacin and levofloxacin, and $\beta$-lactams, such as piperacillin against strains of $E$. coli that presented AcrAB-TolC efflux pumps [28, 141]. In $P$. aeruginosa, although compound $\mathbf{1 2 7}$ presents activity against RND-type pumps, it is unable to penetrate the outer membrane, which is highly selective [28].

In E. coli, the target of compound $\mathbf{1 2 7}$ is the membrane transporter AcrB. This molecule binds to the hydrophobic trap of the $\mathrm{T}$ protomer, interacting with the hydrophobic residues that constitute the deep binding pocket as a hydrophobic trap. $\pi-\pi$ Interactions also occurs, between the pyridine ring of $\mathbf{1 2 7}$ and the aromatic side chain of Phe-628. The phenyl and morpholinyl groups also interact with Phe178 and Phe-615. The dimethylenesulfide linker and the geminal dimethyl group present van der Waals interactions between the side chain of Phe-178, and Tyr-327 and Met573, respectively [28, 147].

SAR studies demonstrated that the maintenance of activity depended on the geminal dimethyl moiety of the tetrahydropyran, the nitrile group and the length of the dimethylenesulfide linker. This data is presented in Figure 14. On the other hand, the morpholine moiety and aryl group are both prone to substitution, originating more stable derivatives in terms of activity, solubility, and metabolism [28]. Studies with this scaffold have shown that non-acidic substituents can be added to the phenyl group, in order to improve potency and CYP450 inhibition, and modifications to the morpholinyl group has greater effects on solubility and stability [140].

Analogues were synthesized by Sjuts et al. (2016), by changing the structure and functional groups of compound 127 (148). They reached the conclusions that the introduction of a 2,6-dimethyl to the morpholinyl group improved both microsomal stability and EPI activity. The replacement of the morpholinyl group with 2methoxyethylpiperazinyl improved aqueous solubility, at the cost of a slight reduction of EPI activity. Two analogues, 128 and 129, resultant of the combination of 2,6dimethylmorpholinyl and acetamide or acrylamide, respectively, on the phenyl group, resulted in a 10-fold to 20fold increase in EPI activity against $E$. coli, respectively, comparing to MBX2319 (127) [28, 148]. The increased EPI activity of these compounds has its basis in the acetamide and acrylamide groups and the morpholinyl group. The acetamide groups are engaged in highly ordered and complex hydrogen bonds, with its center in a solvent water molecule, which plays the role of a hydrogen bond donor to the carbonyl backbone oxygen of Ala-286 and Gln-151 side chain of AcrB. The acrylamide group forms a bridging hydrogen bond through a water molecule to the side chain of Gln-176 [28].

Nguyen et al. (2015) also synthesized a series of analogues, with the morpholine scaffold substituted by a 2,6dimethylmorpholinyl group (140). Many of these analogues exhibited a solubility improvement of 10-fold, compared to compound 127. The most potent and promising compounds 
of these series were compounds 130, 131, 132, 133 and 134 [140].

Through a screening of an $N$-heterocyclic organic compound library, Bohnert and Kern (2005) were able to find a novel class of EPIs, the arylpiperazines [25, 149]. The aim of their work was to find compounds that would potentiate levofloxacin potency against strains of $E$. coli overexpressing $a c r A B$ and acrEF. This was fulfilled when they found phenylpiperazine derivatives with promising activity [25, 149]. SAR studies suggested that elongation of the spacer between the benzene ring and the piperazine ring would enhance potency. Also, substitutions on the benzene ring by halogen atoms led to an increase in potency [24, 149]. On the other hand, the potency would decrease when an ethyl or phenyl group was added to the piperazine ring. With this, the authors found the most potent phenylpiperazine, meta-TFMPP (135), and naphthylpiperazine, 1-(1-naphthylmethyl)-piperazine (NMP) (136) [149].

NMP (136) was the most potent unsubstituted arylpiperazine, able to increase the intracellular concentration of chloramphenicol, tetracycline, linezolid, fluoroquinolones, and macrolides. Although it does not display action in $P$. aeruginosa, it shows effectiveness in $A$. baumanii and several Enterobacteriaceae [11, 23]. EPI activity was also reported in clinical isolates of $E$. coli, most relevantly in fluoroquinolone resistance [150]. Through computational analysis, it was found that NMP (136) binds with high affinity to the lower part of the deep pocket of the $\mathrm{B}$ monomer, overlapping in part the binding site of chloramphenicol [11]. The binding site for NMP (136) includes interactions with hydrophobic residues near the hydrophobic patch and Gly-617 of the G-loop. This data suggests that NMP (136) interferes with the movement of the G-loop, important for extruding substrates, thus inhibiting the action of AcrB [25].

Nakayama et al. (2003) performed a series of syntheses in order to attain a compound specific for the inhibition of a MexAB-OprM specific EPI [151-152]. They identified a hit, compound 137, through high-throughput screening for levofloxacin potentiation using a strain of $P$. aeruginosa strain overexpressing MexAB-OprM. The features this group was looking for was the effective inhibition of efflux pump and the lack of intrinsic antibacterial activity, since efflux pumps are not vital for the organism's survival [151-152]. It was shown that this compound effectively potentiated the activity of levofloxacin, leading to an 8-fold decrease in the antibiotic's MIC. However, its physicochemical properties were problematic: water solubility was poor and it presented high affinity to serum albumin. Therefore, they divided the molecule into three parts, A, B, and C (137) and looked for alternative scaffolds and substituted polar groups, in order to reduce serum protein binding [152].

It was shown that when a carboxamide was introduced in $\mathrm{A}$, the affinity to serum albumin was diminished, as was its activity. More polar moieties led to a complete loss of activity. The strategy for the styrene portion (B) that was the most successful was to substitute the benzene ring to a 3,5 - disubstituted pyridine, which had both good activity and no major serum influence. As for the vinyl group in B, it was demonstrated that molecules bearing methyloxy and ethylene moieties displayed favorable activity profiles. As for part $\mathrm{C}$, the basis of the modifications was the fact that the introduction of bulky or hydrophilic groups adjacent to the carboxyl group would reduce protein binding. An enantiomeric pair containing a dioxolane moiety presented both activity and serum stability, being the $S$ enantiomer more active [152].

Regarding the general scaffold, new compounds were designed, keeping in mind that the thiazole moiety was crucial for activity. Therefore, a molecule having a thiazole side chain attached to the $\mathrm{C}-7$ position of a quinolone was synthesized. Substitution with a tetrazole portion yielded better results than substitution with a carboxylic acid. One compound (138) presented the best activity and serum stability [153].

Further efforts were placed in order to increase the potency of this compound. The in vitro activity was enhanced by the introduction of a hydrophobic group at the 2-position of the pyridopyrimidine scaffold, and hydrophilic substitution would not compromise the activity. The most promising compound had the inclusion of an olefin spacer between the tetrazole and the pyridopyrimidine scaffold, with the limitation of being prone to photoisomerization [154]. To circumvent this, the ethylene tether between the pyridopyrimidine was replaced with an amide bond, providing stability against photoisomerization and, unexpectedly, improving potency. However, another problem arose: the lack of solubility for intravenous use [155]. The following step was the introduction of a secondary amine, giving a zwitterionic analogue, with retention of activity. C-2 Analogues, namely with cyclic substituents, also showed promising results [156]. Once again, the problem of solubility arose. Therefore, efforts were placed in making the molecule more hydrophilic, with the introduction of substituted aromatic moieties. Analogues 4-substituted were the most potent, and the introduction of a morpholine moiety led to increased solubility, maintaining the serum stability. Compound $\mathbf{1 3 9}$ was the most balanced molecule in terms of the desired features [157]. The last refinement made was the introduction of a quaternary ammonium salt, which led to D13-9001 (140). Adding to its high solubility and safety, it was able to potentiate the activity of aztreonam [158]. Figure 15 illustrates the SAR established for 140 and derivatives.

3D Crystal structures of $\mathbf{1 4 0}$ bound to the target, either AcrB or MexB, show that the tert-butyl thiazolyl aminocarboxyl pyridopyrimidine moiety of this compound binds tightly to a narrow depression, designated by hydrophobic trap, close to the deep substrate binding pocket. Additionally, the tetrazole ring and the piperidine acetoamino ethylene ammonioacetate moiety interact with ionic and/or hydrophilic residues in the substrate translocation channel. The piperidine acetoamino ethylene ammonio-acetate moiety also covers part of the minocycline and doxorubicin binding site $[23,28$, $151]$. 
Quinoline derivatives with EPI activity include a multiplicity of derivatives, including pyrrido-, alkoxy-, thioalkoxy-, alkylamino- and chloro- derivatives. These compounds were able to restore an intracellular concentration of antibiotic drugs expelled by efflux pumps, as well as to induce the increase in antibiotic susceptibility, acting as competitive inhibitors of the antibiotic flux. In E. aerogenes and $K$. pneumoniae, quinolines were more effective than $\mathrm{PA} \beta \mathrm{N}$ (124) in inhibiting the activity of the AcrB pump, potentiating the activity of fluoroquinolones, tetracycline, and chloramphenicol [11, 24, 26]. Among the different classes of substituted quinolines, it was proven that the best side chain was piperidinoethyl, which potentiated efficiently the restoration of drug susceptibility in alkoxy- and thioalkoxyquinolines. The heteroatom connecting the side chain is also crucial for activity, being the most potent the amino substituent, then the thioalkyl-, followed by the oxoderivative. The position of the branched substituted groups is also detrimental for the EPI activity [24]. Branched side chains containing piperidinoethyl and morpholinopropyl groups were associated with the most interesting results, being compound 141, containing a piperidinoethyl side chain, the most active when in combination with chloramphenicol [159]. It has been hypothesized that the pump inhibition is greatly influenced by the branched side chain, and takes place either in the inner-membrane transporter or at the junction between the inner pump and the outer channel [159].

Given these promising results, further research in this kind of compounds was performed. Alkoxyquinolines were synthesized and tested against a strain of E. aerogenes overexpressing the AcrAB efflux pump. Compound 142 induced an increase in the susceptibility of chloramphenicol, tetracycline, and fluoroquinolone. This could happen due to interference during active pumping out of the antibiotic [160].

Chloroquinolines also demonstrated efficacy as EPIs, modulating chloramphenicol activity, decreasing its MIC 8fold for resistant strains. Synthesis of analogues led to the conclusion that amino derivatives are more potent than thiol derivatives, pointing out the importance of the side chain for the efflux inhibition. Protonation of the nitrogen could also play an important role at environmental $\mathrm{pH}$, stimulating recognition and binding of the drug to the sites located within the pump's cavity. The heteroatom is also crucial for the activity, being nitrogen the most efficient. Compound 143 is the chloroquinoline with the best results found for the inhibition of the main efflux pump of E. aerogenes [161]. Alkylaminoquinazoline derivatives lacking a nitro group were also evaluated for their ability to decrease chloramphenicol and quinolone efflux in E. aerogenes, through the inhibition of AcrAB efflux pump. The results obtained suggest that this family of compounds recognizes the same pump site responsible for the transport of chloramphenicol and quinolones, competing with them. Structurally, it has been shown that a morpholine group with a propyl chain makes a more active alkylaminoquinazoline.
The most active compound of this family is compound 144 [162].

Benzothiazoles 2-substituted were first tested for the potentiation of ciprofloxacin in AdeABC overexpressing $A$. baumanii. These compounds did not show antibacterial activity when tested alone in bacteria. However, when combined with ciprofloxacin, there was an observation of a reversal in antibacterial susceptibility. The synthesis of several derivatives of this family led to pharmacophore studies, regarding the features of the most promising compounds. Using computational methods, it was revealed that, in order for the compounds to be biologically active, the following features should be present: the nitrogen atom in the thiazole ring and the carbonyl oxygen in the amide function substituted on the second position of the benzothiazole ring must have an hydrogen bonding acceptor property; the benzene ring in the fused ring system, the phenyl group attached to the second position of the benzothiazole ring and the phenyl ring in the 2phenylacetamide and/or 3-phenylpropionamide moiety are essential, since they provide a hydrophobic aromatic property. Of the synthesized compounds, the ones that gathered these features and, therefore, presented the most promising activity, were compounds 145, 146, and 147 [163]. These compounds were also tested for their activity against AcrAB-TolC, in an overexpressing strain of E. coli, reducing the MIC of ciprofloxacin 10-fold, in the case of 146 and 147, and 8-fold, for 145 [164]. Unlike AdeABC, AcrAB-TolC's crystal structure is elucidated, making docking studies possible. This predicted that all three compounds act as AcrB substrates, binding to the distal pocket site in the AcrB porter domain by blocking or inhibiting the ciprofloxacin binding site, generating steric hindrance and thus impeding the binding of the antibiotic. Furthermore, 146 and 147 showed stronger binding interactions than ciprofloxacin [164].

Indole derivatives have been useful in inhibiting protein targets of virus, tumors and bacteria. Since small molecules with conjugated aromatic rings, in which indoles fit, present high potential as EPIs, efforts were placed in synthesizing derivatives that presented this kind of activity. The TolC 3D structure has already been disclosed, making possible the structure-based design of inhibitors. Indoles 148 and 149 were proven efficient inhibitors of TolC in $E$. coli, potentiating the effects of chloramphenicol, tetracycline, erythromycin, and ciprofloxacin, with decreased MIC values of 2-fold for tetracycline and erythromycin and 8-fold for the other two [165].

\subsubsection{Existing Drugs}

Other drugs used for treatments other than antibacterial have demonstrated EPI activity. Tetracycline resistance in $P$. aeruginosa was reduced 8-fold when the phenothiazine fluphenazine, an antipsychotic, was present. Chlorpromazine (150) also showed EPI-like activity for the BpeAB-OprB system in Burkholderia pseudomallei and a reduction of the expression of $a c r B$ in $E$. coli and $S$. enterica samovar 
Typhimurium, suggesting an inhibition of AcrB production [11, 27, 128].

Selective serotonin-reuptake inhibitors have been studied for their ability to act as EPIs for RND efflux pumps in E. coli. It was found that sertraline (151) was capable of potentiating the activity of fluoroquinolones. However, its activity is limited, since it is possible that this compound also acts as an inductor of efflux pumps. Studies have shown that this compound could accumulate in tissues or cells, and therefore, sertraline's (151) concentration may be higher than that measured in plasma. This means that a standard dose of this drug could enhance antibiotic efficacy. It has not yet been studied if a sertraline (151) metabolite could be administered at a dose higher than sertraline. However, it was hypothesized that it could have a better EPI activity, while causing less undesired effects on the central nervous system [166].

Through the observation of previously reported EPIs, Piddock et al. (2010) suggested that simple heterocyclic nitrogen-containing compounds could also act as EPIs and synergize with ciprofloxacin in strains of $S$. enterica serovar Typhimurium that overexpressed the AcrAB-TolC efflux pump [167]. It has been shown that compounds containing a phenylethylamine moiety, as PA $\beta N$ (126) does, such as epinephrine, norepinephrine, and cathinone, are able to potentiate the activity of ciprofloxacin. Of the compounds tested, trimethoprim (152) and epinephrine (153), showed the best results for the tested strains, which included, apart from $S$. enterica serovar Typhimurium, $P$. aeruginosa, E. coli, and $K$. pneumoniae. These compounds showed synergy for the strains where AcrAB-TolC was active, but showed no activity when the pump was inactive. Thus, it can be hypothesized that these compounds either inhibit the expression of the genes encoding AcrAB-TolC, or interact directly with the pump [167]. Both compounds possess structural features similar to previously described EPIs, such as PA $\beta \mathrm{N}$ (124), L-Orn-L-hPhe-3-NHQ (125), and NMP (136), such as an aromatic ring linked to a basic nitrogen, in the case of epinephrine (153), or a pyrimidine ring, for trimethoprim (152). Trimethoprim (152) is already used in combination with another antibiotic, sulfamethoxazole, and combination with a quinolone was hypothesized to be a viable choice. On the other hand, a combination of epinephrine (153) with an antibiotic does not seem a viable option, because of the likelihood of the occurrence of sympathomimetic effects [167].

The antimalarial artesunate (154) has also shown EPI activity, enhancing the effects of $\beta$-lactam antibiotics, such as penicillin $\mathrm{G}$, oxacillin, and ampicillin, and novobiocin against $E$. coli. The mechanism through which 154 expresses its activity is by the inhibition of the expression of the AcrAB-TolC system, not exhibiting antibiotic activity by itself [168].

\section{SMR}

The SMR family comprises the smallest drug efflux proteins known, possessing only four transmembrane segments in its composition, and are exclusive to bacteria. They are involved in the efflux of lipophilic compounds, such as quaternary ammonium salts and a variety of antibiotics. These pumps also use an electrochemical proton gradient in order to exert its efflux activity [7, 32, 38, 169].

One example of a SMR-type pump is the EmrE transporter (Figure 16, PDB 3B61), present in E. coli. In the absence of the ligand, the asymmetric unit of EmrE contains eight EmrE monomers, with four transmembrane helices composing each of them. If a ligand is present, it can function as an inverted homodimer [7, 170].

There have not been many studies concerning inhibitors of this family of efflux pumps, and the few that have been performed were only in silico. Figure 17 shows the structures of the SMR inhibitors that will be further discussed.

\subsection{SMR inhibitors}

\subsubsection{Natural Products}

Quercetin (155) is a flavonol, present in many plants. This compound is very well tolerated in humans, as are its glycoconjugates, and presents a multiplicity of activities, such as antioxidant, anti-inflammatory and antimycobacterial. Only docking studies of this compound with two pumps belonging to the SMR family, the Mmr, in Mycobacterium smegmatis, and the EmrE pumps, in E. coli, were carried out. In Mmr, the hydroxyl groups present in the 3 ' and 4' positions of the B ring of quercetin were predicted to interact with Glu-14 in the helix 1, and the keto group of the hydroxychromen ring of the ligand interacts with the Trp-48 of the helix 1. Additionally, hydrophobic interactions were shown by the residues Phe-44, Tyr-40 and Trp-48, and $\pi-\pi$ interactions were found with Phe-44. Furthermore, 155 was also predicted of interacting with dimeric forms of the protein [171]. In the case of EmrE, the interaction between the 3' and 4' hydroxyls with Glu-14 can also be observed, as can the hydroxyl in the C-7 position with Tyr-60. Hydrophobic interactions are present, with the residues Ile11, Ser-64, Gly-67 and Leu-70, and $\pi-\pi$ interactions exist with the residues Tyr-60 and Trp-63. Structural studies suggest that EmrE is mostly in the dimer state, which also interacts with quercetin (155) [171].

\subsubsection{Synthetic Compounds}

Cation ligands were subjected to docking studies with the $\mathrm{Mmr}$ protein of the SMR family in Mycobacterium tuberculosis and the EmrE dimer from E. coli. The rationale behind this study is the fact that SMR can extrude cations. This study led to the identification of new virtual leads, amongst which is compound 156. ADME studies prove that all the molecules present in this study obey the Lipinski rule of five and the Jorgenson rule of three, which shows that the compounds possess good drug-like properties [172].

\section{MATE}


MATE transporters are responsible for the efflux of cationic, lipophilic substances, providing bacteria and cancer cells with MDR, using $\mathrm{Na}^{+}$or $\mathrm{H}^{+}$influx. They have 12 transmembrane helices, constituted by 400 to 700 amino acids. The mechanism of efflux is thought to happen by a rocker-switch mechanism. Members of the MATE family include the MepA (Figure 18, PDB 1TZP) transporter, in $S$. aureus, even though seventeen MATE proteins have been identified in eleven species, capable of extruding antibiotics. Ethidium bromide, tetraphenylphosphonium, acriflavine, berberine, and norfloxacin have been reported as MATE substrates [38, 173-181].

In Figure 19 are compiled the structures of the compounds that have so far proven their usefulness as MATE inhibitors.

\subsection{MATE inhibitors}

\subsubsection{Natural Products}

A study performed by Kesherwani et al. (2017) accomplished the docking of phytochemicals with the NorM transporter, belonging to the MATE family [182]. After a screening of a library of natural products, three hits were identified: derhamnosyl suspenaside (157), prunin 7', $O$ gallate (158), and quercetin diglucoside (159). These were the best compounds, as their results in binding free energy calculation were the most favorable. When binding free energy decomposition and hydrogen and hydrophobic statistics were taken into account, $\mathbf{1 5 8}$ was elected the best hit compound. Furthermore, this compound was stable during the simulation. Overall, it has been suggested that the compounds which interact towards the cation binding residue in the central cavity of NorM will have better results, and that good inhibitors should have interaction with a few key residues, Gln-34, Val-35, Gly-38, Phe-63, Tyr-67, Ile68, Glu-261, Tyr-294, and Asp-377, which will lead to maximum stability and affinity with the NorM transporter [182].

\subsubsection{Synthetic Compounds}

These compounds have been previously described as MFS inhibitors, namely of the NorA pump. However, similar activity has been described for the MepA pump, of the MATE family. Compound $\mathbf{8 0}$ (Figure 5), incorporating a bisaryl urea moiety at the 7-position, was able to successfully inhibit approximately $84 \%$ of ethidium bromide efflux in a MepA overexpressing strain of S.aureus [104]. Phenylquinoline derivatives also proved efficient against the same strain. Differently from what was observed with NorA, the compounds that inhibited MepA most efficiently were 86 (Figure 5) and 160 (Figure 19), which were able to reduce ethidium bromide's MIC by 16-fold at concentrations of 0.78 and $1.56 \mu \mathrm{g} / \mathrm{ml}$, respectively, in the MepA overexpressing strain. Ethidium bromide was chosen, instead of ciprofloxacin, as it is a better MepA substrate and other derivatives were able to inhibit both NorA and MepA efflux pumps, being able to completely restore the activity of ciprofloxacin and ethidium bromide against strains overexpressing such pumps [107].

Paroxetine derivatives (104), effective against the NorA pump, also demonstrated potency in inhibiting the MepA pump in $S$. aureus. Oppositely to NorA, paroxetine derivatives do not need the fluorophenyl ring at position 4 to be MepA EPIs. In fact, 4-unsubstituted analogues proved to be more potent than 4-F-phenyl derivatives in inhibiting the MepA pump, as is the case of compound 161 and 162 [122].

Efflux pumps are one of the main causes for antimicrobial resistance. Their inhibition can lead to the restoration of bacteria susceptibility and antibiotic efficacy. Throughout this review, the data presented leads to the conclusion that EPIs are promising compounds and can help reversing the problem of antimicrobial resistance. However, the research into these kind of compounds is still in a very preliminary state, since no EPI is yet under clinical trials.

Even though no general structural features can be pointed out for a molecule to be an EPI, it can be observed that small molecules with high lipophilicity are most likely to fulfill the requirements to inhibit efflux pumps. Hydrophilic compounds can also be transported through porins present in the membrane. Heterocyclic compounds also show potential as EPIs, mainly nitrogen heterocycles, but also fluorine and boron heteroatoms, since the likelihood of bacterial exposure to these atoms is very low. The basis for the MDR pumps inhibition has to do with the penetration rules, and whether or not a compound is capable of penetrating the bacterial membrane. Therefore, a library of compounds that are able to do that would be something that would bring advances to this study field.

One of the main aims of this review is to present SAR studies, so that pharmaceutical chemists can refine molecular structures, leading to the achievement of more potent and specific compounds. The use of synthetic approaches, either to modify molecules obtained from natural sources, or drugs that are already in therapeutic use, or even to develop novel compounds, is vital for these SAR studies. The search of EPIs derived from natural products comes from the fact that plants need to protect themselves from pathogenic microorganisms, and produce metabolites that act as antimicrobial and EPIs, that synergize with each other. However, the referred compounds are not specific for the pump they have been studied, showing a multitarget character: flavonoids, coumarins, cinnamic acids, among others, present a multiplicity of activities already described, suggesting their promiscuity.

The advantage of assays with existing drugs lies in their safety profile and, most times, their clinical use for combination therapy has been characterized. In some cases, the compounds were even investigated as inhibitors of human efflux pumps, for cancer treatment. This can be regarded as an advantage, leading to a new class of drugs with dual human/bacterial EPI, particularly useful in cancer patients with infections, potentiating the effects of both the anticancer and the antibacterial drugs. 
The future in studies in bacterial EPIs should lie either in the search for selectivity for the bacterial target, or in the disclosure of the common features of the universal bacterial EPI, that can successfully target all efflux pumps. The SAR studies gathered herein with the 3D structural information available are expected to accelerate the structure-based design of new EPIs, founded on the existing models.

\section{LIST OF ABBREVIATIONS}

3D-QSAR: Three-dimensional quantitative structure activity relationship; ABC: ATP-binding cassette; ADME: Absorption, distribution, metabolism and excretion; ATP: Adenosine triphosphate; BCRP: Breast cancer resistance protein; COX-2: Ciclooxigenase-2; CYP450: Cytochrome P450; EPI: Efflux pump inhibitor; $\mathrm{IC}_{50}$ : Half maximal inhibitory concentration; MATE: Multidrug and toxic compound extrusion; MDR: Multidrug resistance; MIC: Minimum inhibitory concentration; MFS: Major facilitator superfamily; MRP: Multidrug resistance protein; MRSA: Methicillin-resistant Staphylococcus aureus; NDGA: Nordihydroguaretic acid; NMP: 1-(1-Naphthylmethyl)piperazine; P-gp: P-Glycoprotein; PA $\beta \mathrm{N}$ : Phenyl-arginine $\beta$ naphtylamide; PDB: Protein Data Bank; PSSRI: Phenylpiperidine selective serotonin reuptake inhibitors; RND: Resistance-nodulation-division; SAR: Structure activity relationship; SMR: Small multidrug resistance.

\section{REFERENCES}

[1] Davies, J.; Davies, D., Origins and Evolution of Antibiotic Resistance. Microbiol Mol Biol Rev 2010, 74 (3), 417-433.

[2] Alanis, A. J., Resistance to antibiotics: are we in the postantibiotic era? Arch Med Res 2005, 36 (6), 697-705.

[3] Ventola, C. L., The Antibiotic Resistance Crisis: Part 1: Causes and Threats. P T 2015, 40 (4), 277-283.

[4] Levy, S. B.; Marshall, B., Antibacterial resistance worldwide: causes, challenges and responses. Nat Med 2004, 10 (12 Suppl), S122-9.

[5] Munita, J. M.; Arias, C. A., Mechanisms of Antibiotic Resistance. Microbiol Spect 2016, 4 (2), 10.1128/microbiolspec.VMBF-0016-2015.

[6] Chattopadhyay, M. K.; Jagannadham, M. V., Vesiclesmediated resistance to antibiotics in bacteria. FrontMicrobiol 2015, 6, 758.

[7] Sun, J.; Deng, Z.; Yan, A., Bacterial multidrug efflux pumps: mechanisms, physiology and pharmacological exploitations. Biochem Biophys Res Commun 2014, 453 (2), 254-67.

[8] Blanco, P.; Hernando-Amado, S.; Reales-Calderon, J. A.; Corona, F.; Lira, F.; Alcalde-Rico, M.; Bernardini, A.; Sanchez, M. B.; Martinez, J. L., Bacterial Multidrug Efflux Pumps: Much More Than Antibiotic Resistance Determinants. Microorganisms 2016, 4 (1).
[9] Fernandez, L.; Hancock, R. E., Adaptive and mutational resistance: role of porins and efflux pumps in drug resistance. Clin Microbiol Rev 2012, 25 (4), 661-81.

[10] Webber, M. A.; Piddock, L. J. V., The importance of efflux pumps in bacterial antibiotic resistance. J Antimicrob Chemother 2003, 51 (1), 9-11.

[11] Tegos, G. P.; Haynes, M.; Strouse, J. J.; Khan, M. M.; Bologa, C. G.; Oprea, T. I.; Sklar, L. A., Microbial efflux pump inhibition: tactics and strategies. Curr Pharm Des 2011, 17 (13), 1291-302.

[12] Van Bambeke, F.; Pages, J. M.; Lee, V. J., Inhibitors of bacterial efflux pumps as adjuvants in antibiotic treatments and diagnostic tools for detection of resistance by efflux. Recent Pat Antiinfect Drug Discov 2006, 1 (2), 157-75.

[13] Marquez, B., Bacterial efflux systems and efflux pumps inhibitors. Biochimie 2005, 87 (12), 1137-47.

[14] Kumar, R.; Patial, S., A Review on Efflux Pump Inhibitors of Gram-Positive and Gram-Negative Bacteria from Plant Sources. Int J Curr Microbiol App Sci 2016, 5 (6), 834-855.

[15] Rana, T.; Singh, S.; Kaur, N.; Pathania, K.; Farooq, U., A Review on Efflux Pump Inhibitors of Medically Important Bacteria from Plant Sources. Int J Pharm Sci Rev Res 2014, 26 (2), 101-111.

[16] Prasch, S.; Bucar, F., Plant derived inhibitors of bacterial efflux pumps: an update. Phytochem Rev 2015, 14 (6), 961-974.

[17] Zhang, L.; Ma, S., Efflux pump inhibitors: a strategy to combat P-glycoprotein and the NorA multidrug resistance pump. Chem Med Chem 2010, 5 (6), 811-22.

[18] Schindler, B. D.; Jacinto, P.; Kaatz, G. W., Inhibition of drug efflux pumps in Staphylococcus aureus: current status of potentiating existing antibiotics. Future Microbiol 2013, 8 (4), 491-507.

[19] Kumar, S.; Mukherjee, M. M.; Varela, M. F., Modulation of Bacterial Multidrug Resistance Efflux Pumps of the Major Facilitator Superfamily. Int J Bacteriol 2013, 2013.

[20] Handzlik, J.; Matys, A.; Kiec-Kononowicz, K., Recent Advances in Multi-Drug Resistance (MDR) Efflux Pump Inhibitors of Gram-Positive Bacteria S. aureus. Antibiotics (Basel) 2013, 2 (1), 28-45.

[21] Stavri, M.; Piddock, L. J. V.; Gibbons, S., Bacterial efflux pump inhibitors from natural sources. $J$ Antimicrob Chemother 2007, 59 (6), 1247-1260.

[22] Gibbons, S., Plants as a Source of Bacterial Resistance Modulators and Anti-Infective Agents. Phytochem Rev 2005, 4 (1), 63-78.

[23] Ruggerone, P.; Murakami, S.; Pos, K. M.; Vargiu, A. V., RND efflux pumps: structural information translated into function and inhibition mechanisms. Curr Top Med Chem 2013, 13 (24), 3079-100.

[24] Pagès, J.-M.; Masi, M.; Barbe, J., Inhibitors of efflux pumps in Gram-negative bacteria. Trends Mol Med 2005, 11 (8), 382-389.

[25] Opperman, T.; Nguyen, S., Recent advances toward a molecular mechanism of efflux pump inhibition. Front Microbiol 2015, 6 (421). 
[26] Martins, M.; Dastidar, S. G.; Fanning, S.; Kristiansen, J. E.; Molnar, J.; Pages, J. M.; Schelz, Z.; Spengler, G.; Viveiros, M.; Amaral, L., Potential role of non-antibiotics (helper compounds) in the treatment of multidrug-resistant Gram-negative infections: mechanisms for their direct and indirect activities. Int $J$ Antimicrob Agents 2008, 31 (3), 198208.

[27] Blair, J. M. A.; Piddock, L. J. V., Structure, function and inhibition of RND efflux pumps in Gram-negative bacteria: an update. Curr Opin Microbiol 2009, 12 (5), 512519.

[28] Aron, Z.; Opperman, T. J., Optimization of a novel series of pyranopyridine RND efflux pump inhibitors. Curr Opin Microbiol 2016, 33, 1-6.

[29] Venter, H.; Mowla, R.; Ohene-Agyei, T.; Ma, S., RNDtype drug efflux pumps from Gram-negative bacteria: molecular mechanism and inhibition. Front Microbiol 2015, $6,377$.

[30] Song, L.; Wu, X., Development of efflux pump inhibitors in antituberculosis therapy. Int $J$ Antimicrob Agents 2016, 47 (6), 421-9.

[31] Pule, C. M.; Sampson, S. L.; Warren, R. M.; Black, P. A.; van Helden, P. D.; Victor, T. C.; Louw, G. E., Efflux pump inhibitors: targeting mycobacterial efflux systems to enhance TB therapy. J Antimicrob Chemother 2016, 71 (1), 17-26.

[32] Zechini, B.; Versace, I., Inhibitors of multidrug resistant efflux systems in bacteria. Recent Pat Antiinfect Drug Discov 2009, 4 (1), 37-50.

[33] El-Awady, R.; Saleh, E.; Hashim, A.; Soliman, N.; Dallah, A.; Elrasheed, A.; Elakraa, G., The Role of Eukaryotic and Prokaryotic ABC Transporter Family in Failure of Chemotherapy. Front Pharmacol 2016, 7, 535.

[34] Silva, R.; Vilas-Boas, V.; Carmo, H.; Dinis-Oliveira, R. J.; Carvalho, F.; de Lourdes Bastos, M.; Remião, F., Modulation of P-glycoprotein efflux pump: induction and activation as a therapeutic strategy. Pharmacol Ther 2015, 149, 1-123.

[35] Palmeira, A.; Rodrigues, F.; Sousa, E.; Pinto, M.; Vasconcelos, M. H.; Fernandes, M. X., New uses for old drugs: pharmacophore-based screening for the discovery of P-glycoprotein inhibitors. Chem Biol Drug Des 2011, 78 (1), 57-72.

[36] Lopes-Rodrigues, V.; Sousa, E.; Vasconcelos, M. H., Curcumin as a Modulator of P-Glycoprotein in Cancer: Challenges and Perspectives. Pharmaceuticals (Basel) 2016, 9 (4).

[37] Lopes-Rodrigues, V.; Seca, H.; Sousa, D.; Sousa, E.; Lima, R. T.; Vasconcelos, M. H., The network of Pglycoprotein and microRNAs interactions. Int $J$ Cancer 2014, 135 (2), 253-63.

[38] Jang, S., Multidrug efflux pumps in Staphylococcus aureus and their clinical implications. J Microbiol 2016, 54 (1), 1-8.

[39] Locher, K. P., Mechanistic diversity in ATP-binding cassette (ABC) transporters. Nat Struct Mol Biol 2016, 23 (6), 487-93.
[40] Wilkens, S., Structure and mechanism of ABC transporters. F1000Prime Rep 2015, 7, 14.

[41] Taylor, A. B.; Benglis, J. D. M.; Dhandayuthapani, S.; Hart, P. J., Structure of Mycobacterium tuberculosis Methionine Sulfoxide Reductase A in Complex with ProteinBound Methionine. J Bacteriol 2003, 185 (14), 4119-4126. [42] Oluwatuyi, M.; Kaatz, G. W.; Gibbons, S., Antibacterial and resistance modifying activity of Rosmarinus officinalis. Phytochemistry 2004, 65 (24), 3249-54.

[43] Hussein, A. A.; Rodriguez, B., Isopimarane diterpenoids from Lycopus europaeus. J Nat Prod 2000, 63 (3), 419-21.

[44] Gibbons, S.; Oluwatuyi, M.; Veitch, N. C.; Gray, A. I., Bacterial resistance modifying agents from Lycopus europaeus. Phytochemistry 2003, 62 (1), 83-7.

[45] Upadhyay, H. C.; Dwivedi, G. R.; Roy, S.; Sharma, A.; Darokar, M. P.; Srivastava, S. K., Phytol derivatives as drug resistance reversal agents. Chem Med Chem 2014, 9 (8), 1860-8.

[46] Dwivedi, G. R.; Maurya, A.; Yadav, D. K.; Khan, F.; Darokar, M. P.; Srivastava, S. K., Drug Resistance Reversal Potential of Ursolic Acid Derivatives against Nalidixic Acidand Multidrug-resistant Escherichia coli. Chem Biol Drug Des 2015, 86 (3), 272-83.

[47] Wang, S. Y.; Sun, Z. L.; Liu, T.; Gibbons, S.; Zhang, W. J.; Qing, M., Flavonoids from Sophora moorcroftiana and their synergistic antibacterial effects on MRSA. Phytother Res 2014, 28 (7), 1071-6.

[48] Chan, B. C.; Ip, M.; Gong, H.; Lui, S. L.; See, R. H.; Jolivalt, C.; Fung, K. P.; Leung, P. C.; Reiner, N. E.; Lau, C. B., Synergistic effects of diosmetin with erythromycin against ABC transporter over-expressed methicillin-resistant Staphylococcus aureus (MRSA) RN4220/pUL5054 and inhibition of MRSA pyruvate kinase. Phytomedicine 2013, 20 (7), 611-4.

[49] Dwivedi, G. R.; Upadhyay, H. C.; Yadav, D. K.; Singh, V.; Srivastava, S. K.; Khan, F.; Darmwal, N. S.; Darokar, M. P., 4-Hydroxy- $\alpha$-tetralone and its derivative as drug resistance reversal agents in multi drug resistant Escherichia coli. Chem Biol Drug Des 2014, 83 (4), 482-92.

[50] Chan, B. C.; Han, X. Q.; Lui, S. L.; Wong, C. W.; Wang, T. B.; Cheung, D. W.; Cheng, S. W.; Ip, M.; Han, S. Q.; Yang, X. S.; Jolivalt, C.; Lau, C. B.; Leung, P. C.; Fung, K. P., Combating against methicillin-resistant Staphylococcus aureus - two fatty acids from Purslane (Portulaca oleracea L.) exhibit synergistic effects with erythromycin. J Pharm Pharmacol 2015, 67 (1), 107-16.

[51] Maurya, A.; Dwivedi, G. R.; Darokar, M. P.; Srivastava, S. K., Antibacterial and synergy of clavine alkaloid lysergol and its derivatives against nalidixic acid-resistant Escherichia coli. Chem Biol Drug Des 2013, 81 (4), 484-90. [52] Gibbons, S.; Oluwatuyi, M.; Kaatz, G. W., A novel inhibitor of multidrug efflux pumps in Staphylococcus aureus. J Antimicrob Chemother 2003, 51 (1), 13-7.

[53] Pezza, R. J.; Villarreal, M. A.; Montich, G. G.; Argaraña, C. E., Vanadate inhibits the ATPase activity and DNA binding capability of bacterial MutS. A structural 
model for the vanadate-MutS interaction at the Walker A motif. Nucleic Acids Res 2002, 30 (21), 4700-4708.

[54] Yan, N., Structural advances for the major facilitator superfamily (MFS) transporters. Trends in Biochem Sci 2013, 38 (3), 151-159.

[55] Yin, Y.; He, X.; Szewczyk, P.; Nguyen, T.; Chang, G., Structure of the Multidrug Transporter EmrD from Escherichia coli. Science (New York, N.Y.) 2006, 312 (5774), 741-744.

[56] Yan, N., Structural Biology of the Major Facilitator Superfamily Transporters. Annu Rev Biophys 2015, 44, 25783.

[57] Tamura, N.; Konishi, S.; Yamaguchi, A., Mechanisms of drug/H+ antiport: complete cysteine-scanning mutagenesis and the protein engineering approach. Curr Opin Chem Biol 2003, 7 (5), 570-579.

[58] Coelho, M. L.; Ferreira, J. H.; de Siqueira Junior, J. P.; Kaatz, G. W.; Barreto, H. M.; de Carvalho Melo Cavalcante, A. A., Inhibition of the NorA multi-drug transporter by oxygenated monoterpenes. Microb Pathog 2016, 99, 173177.

[59] Thota, N.; Koul, S.; Reddy, M. V.; Sangwan, P. L.; Khan, I. A.; Kumar, A.; Raja, A. F.; Andotra, S. S.; Qazi, G. N., Citral derived amides as potent bacterial NorA efflux pump inhibitors. Bioorg Med Chem 2008, 16 (13), 6535-43. [60] Smith, E. C.; Williamson, E. M.; Wareham, N.; Kaatz, G. W.; Gibbons, S., Antibacterials and modulators of bacterial resistance from the immature cones of Chamaecyparis lawsoniana. Phytochemistry 2007, 68 (2), 210-7.

[61] Smith, E. C.; Kaatz, G. W.; Seo, S. M.; Wareham, N.; Williamson, E. M.; Gibbons, S., The phenolic diterpene totarol inhibits multidrug efflux pump activity in Staphylococcus aureus. Antimicrob Agents Chemother 2007, 51 (12), 4480-3.

[62] Zhang, J.; Sun, Y.; Wang, Y.; Lu, M.; He, J.; Liu, J.; Chen, Q.; Zhang, X.; Zhou, F.; Wang, G.; Sun, X., Nonantibiotic agent ginsenoside $20(S)$ - $\mathrm{Rh} 2$ enhanced the antibacterial effects of ciprofloxacin in vitro and in vivo as a potential NorA inhibitor. Eur J Pharmacol 2014, 740, 27784.

[63] Zhou, S.; Schuetz, J. D.; Bunting, K. D.; Colapietro, A. M.; Sampath, J.; Morris, J. J.; Lagutina, I.; Grosveld, G. C.; Osawa, M.; Nakauchi, H.; Sorrentino, B. P., The ABC transporter Bcrp1/ABCG2 is expressed in a wide variety of stem cells and is a molecular determinant of the sidepopulation phenotype. Nat Med 2001, 7 (9), 1028-34.

[64] Doyle, L.; Ross, D. D., Multidrug resistance mediated by the breast cancer resistance protein BCRP (ABCG2). Oncogene 2003, 22 (47), 7340-58.

[65] Ahmed, M.; Borsch, C. M.; Neyfakh, A. A.; Schuldiner, S., Mutants of the Bacillus subtilis multidrug transporter Bmr with altered sensitivity to the antihypertensive alkaloid reserpine. J Biol Chem 1993, 268 (15), 11086-9.

[66] Schmitz, F. J.; Fluit, A. C.; Luckefahr, M.; Engler, B.; Hofmann, B.; Verhoef, J.; Heinz, H. P.; Hadding, U.; Jones, M. E., The effect of reserpine, an inhibitor of multidrug efflux pumps, on the in vitro activities of ciprofloxacin, sparfloxacin and moxifloxacin against clinical isolates of Staphylococcus aureus. J Antimicrob Chemother 1998, 42 (6), 807-10.

[67] Markham, P. N.; Westhaus, E.; Klyachko, K.; Johnson, M. E.; Neyfakh, A. A., Multiple Novel Inhibitors of the NorA Multidrug Transporter of Staphylococcus aureus. Antimicrob Agents Chemother 1999, 43 (10), 2404-8.

[68] Kumar, A.; Khan, I. A.; Koul, S.; Koul, J. L.; Taneja, S. C.; Ali, I.; Ali, F.; Sharma, S.; Mirza, Z. M.; Kumar, M.; Sangwan, P. L.; Gupta, P.; Thota, N.; Qazi, G. N., Novel structural analogues of piperine as inhibitors of the NorA efflux pump of Staphylococcus aureus. $J$ Antimicrob Chemother 2008, 61 (6), 1270-6.

[69] Sangwan, P. L.; Koul, J. L.; Koul, S.; Reddy, M. V.; Thota, N.; Khan, I. A.; Kumar, A.; Kalia, N. P.; Qazi, G. N., Piperine analogs as potent Staphylococcus aureus NorA efflux pump inhibitors. Bioorg Med Chem 2008, 16 (22), 9847-57.

[70] Nargotra, A.; Sharma, S.; Koul, J. L.; Sangwan, P. L.; Khan, I. A.; Kumar, A.; Taneja, S. C.; Koul, S., Quantitative structure activity relationship (QSAR) of piperine analogs for bacterial NorA efflux pump inhibitors. Eur J Med Chem 2009, 44 (10), 4128-35.

[71] Stermitz, F. R.; Lorenz, P.; Tawara, J. N.; Zenewicz, L. A.; Lewis, K., Synergy in a medicinal plant: antimicrobial action of berberine potentiated by 5'-methoxyhydnocarpin, a multidrug pump inhibitor. Proc Natl Acad Sci U S A 2000, 97 (4), 1433-7.

[72] Guz, N. R.; Stermitz, F. R.; Johnson, J. B.; Beeson, T. D.; Willen, S.; Hsiang, J.; Lewis, K., Flavonolignan and flavone inhibitors of a Staphylococcus aureus multidrug resistance pump: structure-activity relationships. $J$ Med Chem 2001, 44 (2), 261-8.

[73] Bame, J. R.; Graf, T. N.; Junio, H. A.; Bussey, R. O., 3rd; Jarmusch, S. A.; El-Elimat, T.; Falkinham, J. O., 3rd; Oberlies, N. H.; Cech, R. A.; Cech, N. B., Sarothrin from Alkanna orientalis is an antimicrobial agent and efflux pump inhibitor. Planta Med 2013, 79 (5), 327-9.

[74] Morel, C.; Stermitz, F. R.; Tegos, G.; Lewis, K., Isoflavones as potentiators of antibacterial activity. J Agric Food Chem 2003, 51 (19), 5677-9.

[75] Chan, B. C.; Ip, M.; Lau, C. B.; Lui, S. L.; Jolivalt, C.; Ganem-Elbaz, C.; Litaudon, M.; Reiner, N. E.; Gong, H.; See, R. H.; Fung, K. P.; Leung, P. C., Synergistic effects of baicalein with ciprofloxacin against NorA over-expressed methicillin-resistant Staphylococcus aureus (MRSA) and inhibition of MRSA pyruvate kinase. J Ethnopharmacol 2011, 137 (1), 767-73.

[76] Maia, G. L.; Falcao-Silva Vdos, S.; Aquino, P. G.; de Araujo-Junior, J. X.; Tavares, J. F.; da Silva, M. S.; Rodrigues, L. C.; de Siqueira-Junior, J. P.; Barbosa-Filho, J. M., Flavonoids from Praxelis clematidea R.M. King and Robinson modulate bacterial drug resistance. Molecules 2011, 16 (6), 4828-35.

[77] Falcao-Silva, V. S.; Silva, D. A.; Souza Mde, F.; Siqueira-Junior, J. P., Modulation of drug resistance in Staphylococcus aureus by a kaempferol glycoside from 
Herissantia tiubae (Malvaceae). Phytother Res 2009, 23 (10), 1367-70.

[78] Holler, J. G.; Christensen, S. B.; Slotved, H. C.; Rasmussen, H. B.; Guzman, A.; Olsen, C. E.; Petersen, B.; Molgaard, P., Novel inhibitory activity of the Staphylococcus aureus NorA efflux pump by a kaempferol rhamnoside isolated from Persea lingue Nees. J Antimicrob Chemother 2012, 67 (5), 1138-44.

[79] Holler, J. G.; Slotved, H. C.; Molgaard, P.; Olsen, C. E.; Christensen, S. B., Chalcone inhibitors of the NorA efflux pump in Staphylococcus aureus whole cells and enriched everted membrane vesicles. Bioorg Med Chem 2012, 20 (14), 4514-21.

[80] Belofsky, G.; Percivill, D.; Lewis, K.; Tegos, G. P.; Ekart, J., Phenolic metabolites of Dalea versicolor that enhance antibiotic activity against model pathogenic bacteria. J Nat Prod 2004, 67 (3), 481-4.

[81] Stermitz, F. R.; Tawara-Matsuda, J.; Lorenz, P.; Mueller, P.; Zenewicz, L.; Lewis, K., 5'Methoxyhydnocarpin-D and pheophorbide A: Berberis species components that potentiate berberine growth inhibition of resistant Staphylococcus aureus. J Nat Prod 2000, 63 (8), 1146-9.

[82] Pereda-Miranda, R.; Kaatz, G. W.; Gibbons, S., Polyacylated oligosaccharides from medicinal Mexican morning glory species as antibacterials and inhibitors of multidrug resistance in Staphylococcus aureus. J Nat Prod 2006, 69 (3), 406-9.

[83] Cherigo, L.; Pereda-Miranda, R.; Fragoso-Serrano, M.; Jacobo-Herrera, N.; Kaatz, G. W.; Gibbons, S., Inhibitors of bacterial multidrug efflux pumps from the resin glycosides of Ipomoea murucoides. J Nat Prod 2008, 71 (6), 1037-45.

[84] Stermitz, F. R.; Cashman, K. K.; Halligan, K. M.; Morel, C.; Tegos, G. P.; Lewis, K., Polyacylated neohesperidosides From Geranium caespitosum: bacterial multidrug resistance pump inhibitors. Bioorg Med Chem Lett 2003, 13 (11), 1915-1918.

[85] Kalia, N. P.; Mahajan, P.; Mehra, R.; Nargotra, A.; Sharma, J. P.; Koul, S.; Khan, I. A., Capsaicin, a novel inhibitor of the NorA efflux pump, reduces the intracellular invasion of Staphylococcus aureus. J Antimicrob Chemother 2012, 67 (10), 2401-8.

[86] Fiamegos, Y. C.; Kastritis, P. L.; Exarchou, V.; Han, H.; Bonvin, A. M. J. J.; Vervoort, J.; Lewis, K.; Hamblin, M. R.; Tegos, G. P., Antimicrobial and Efflux Pump Inhibitory Activity of Caffeoylquinic Acids from Artemisia absinthium against Gram-Positive Pathogenic Bacteria. PLOS ONE 2011, 6 (4), e18127.

[87] Shiu, W. K.; Malkinson, J. P.; Rahman, M. M.; Curry, J.; Stapleton, P.; Gunaratnam, M.; Neidle, S.; Mushtaq, S.; Warner, M.; Livermore, D. M.; Evangelopoulos, D.; Basavannacharya, C.; Bhakta, S.; Schindler, B. D.; Seo, S. M.; Coleman, D.; Kaatz, G. W.; Gibbons, S., A new plantderived antibacterial is an inhibitor of efflux pumps in Staphylococcus aureus. Int J Antimicrob Agents 2013, 42 (6), 513-8.

[88] Costa, L. M.; de Macedo, E. V.; Oliveira, F. A.; Ferreira, J. H.; Gutierrez, S. J.; Pelaez, W. J.; Lima, F. C.; de
Siqueira Junior, J. P.; Coutinho, H. D.; Kaatz, G. W.; de Freitas, R. M.; Barreto, H. M., Inhibition of the NorA efflux pump of Staphylococcus aureus by synthetic riparins. J Appl Microbiol 2016, 121 (5), 1312-1322.

[89] Michalet, S.; Cartier, G.; David, B.; Mariotte, A. M.; Dijoux-franca, M. G.; Kaatz, G. W.; Stavri, M.; Gibbons, S., $N$-caffeoylphenalkylamide derivatives as bacterial efflux pump inhibitors. Bioorg Med Chem Lett 2007, 17 (6), 1755 8.

[90] Abulrob, A. N.; Suller, M. T.; Gumbleton, M.; Simons, C.; Russell, A. D., Identification and biological evaluation of grapefruit oil components as potential novel efflux pump modulators in methicillin-resistant Staphylococcus aureus bacterial strains. Phytochemistry 2004, 65 (22), 3021-7.

[91] Roy, S. K.; Kumari, N.; Pahwa, S.; Agrahari, U. C.; Bhutani, K. K.; Jachak, S. M.; Nandanwar, H., NorA efflux pump inhibitory activity of coumarins from Mesua ferrea. Fitoterapia 2013, 90, 140-50.

[92] Oliveira, A. S.; Sousa, E.; Vasconcelos, M. H.; Pinto, M., Curcumin: A Natural Lead for Potential New Drug Candidates. Curr Med Chem 2015, 22 (36), 4196-232.

[93] Lopes-Rodrigues, V.; Oliveira, A.; Correia-da-Silva, M.; Pinto, M.; Lima, R. T.; Sousa, E.; Vasconcelos, M. H., A novel curcumin derivative which inhibits P-glycoprotein, arrests cell cycle and induces apoptosis in multidrug resistance cells. Bioorg Med Chem 2017, 25 (2), 581-596.

[94] Joshi, P.; Singh, S.; Wani, A.; Sharma, S.; Jain, S. K.; Singh, B.; Gupta, B. D.; Satti, N. K.; Koul, S.; Khan, I. A.; Kumar, A.; Bharate, S. B.; Vishwakarma, R. A., Osthol and curcumin as inhibitors of human Pgp and multidrug efflux pumps of Staphylococcus aureus: reversing the resistance against frontline antibacterial drugs. Med Chem Comm 2014, 5 (10), 1540-1547.

[95] Marquez, B.; Neuville, L.; Moreau, N. J.; Genet, J. P.; dos Santos, A. F.; Cano de Andrade, M. C.; Sant'Ana, A. E., Multidrug resistance reversal agent from Jatropha elliptica. Phytochemistry 2005, 66 (15), 1804-11.

[96] Belofsky, G.; Carreno, R.; Lewis, K.; Ball, A.; Casadei, G.; Tegos, G. P., Metabolites of the "smoke tree", Dalea spinosa, potentiate antibiotic activity against multidrugresistant Staphylococcus aureus. J Nat Prod 2006, 69 (2), 261-4.

[97] Ambrus, J. I.; Kelso, M. J.; Bremner, J. B.; Ball, A. R.; Casadei, G.; Lewis, K., Structure-activity relationships of 2aryl-1H-indole inhibitors of the NorA efflux pump in Staphylococcus aureus. Bioorg Med Chem Lett 2008, 18 (15), 4294-7.

[98] Samosorn, S.; Bremner, J. B.; Ball, A.; Lewis, K., Synthesis of functionalized 2-aryl-5-nitro- $1 H$-indoles and their activity as bacterial NorA efflux pump inhibitors. Bioorg Med Chem 2006, 14 (3), 857-65.

[99] Ball, A. R.; Casadei, G.; Samosorn, S.; Bremner, J. B.; Ausubel, F. M.; Moy, T. I.; Lewis, K., Conjugating berberine to a multidrug efflux pump inhibitor creates an effective antimicrobial. ACS Chem Biol 2006, 1 (9), 594-600.

[100] Fournier dit Chabert, J.; Marquez, B.; Neville, L.; Joucla, L.; Broussous, S.; Bouhours, P.; David, E.; PelletRostaing, S.; Marquet, B.; Moreau, N.; Lemaire, M., 
Synthesis and evaluation of new arylbenzo $[b]$ thiophene and diarylthiophene derivatives as inhibitors of the NorA multidrug transporter of Staphylococcus aureus. Bioorg Med Chem 2007, 15 (13), 4482-4497.

[101] Samosorn, S.; Tanwirat, B.; Muhamad, N.; Casadei, G.; Tomkiewicz, D.; Lewis, K.; Suksamrarn, A.; Prammananan, T.; Gornall, K. C.; Beck, J. L.; Bremner, J. B., Antibacterial activity of berberine-NorA pump inhibitor hybrids with a methylene ether linking group. Bioorg Med Chem 2009, 17 (11), 3866-72.

[102] Buonerba, F.; Lepri, S.; Goracci, L.; Schindler, B. D.; Seo, S. M.; Kaatz, G. W.; Cruciani, G., Improved Potency of Indole-Based NorA Efflux Pump Inhibitors: From Serendipity toward Rational Design and Development. $J$ Med Chem 2017, 60 (1), 517-523.

[103] Lepri, S.; Buonerba, F.; Goracci, L.; Velilla, I.; Ruzziconi, R.; Schindler, B. D.; Seo, S. M.; Kaatz, G. W.; Cruciani, G., Indole Based Weapons to Fight Antibiotic Resistance: A Structure-Activity Relationship Study. J Med Chem 2016, 59 (3), 867-91.

[104] German, N.; Wei, P.; Kaatz, G. W.; Kerns, R. J., Synthesis and evaluation of fluoroquinolone derivatives as substrate-based inhibitors of bacterial efflux pumps. Eur $J$ Med Chem 2008, 43 (11), 2453-63.

[105] Pieroni, M.; Dimovska, M.; Brincat, J. P.; Sabatini, S.; Carosati, E.; Massari, S.; Kaatz, G. W.; Fravolini, A., From 6-aminoquinolone antibacterials to 6-amino-7thiopyranopyridinylquinolone ethyl esters as inhibitors of Staphylococcus aureus multidrug efflux pumps. J Med Chem 2010, 53 (11), 4466-80.

[106] Sabatini, S.; Gosetto, F.; Manfroni, G.; Tabarrini, O.; Kaatz, G. W.; Patel, D.; Cecchetti, V., Evolution from a natural flavones nucleus to obtain 2-(4Propoxyphenyl)quinoline derivatives as potent inhibitors of the $S$. aureus NorA efflux pump. J Med Chem 2011, 54 (16), 5722-36.

[107] Sabatini, S.; Gosetto, F.; Iraci, N.; Barreca, M. L.; Massari, S.; Sancineto, L.; Manfroni, G.; Tabarrini, O.; Dimovska, M.; Kaatz, G. W.; Cecchetti, V., Re-evolution of the 2-phenylquinolines: ligand-based design, synthesis, and biological evaluation of a potent new class of Staphylococcus aureus NorA efflux pump inhibitors to combat antimicrobial resistance. J Med Chem 2013, 56 (12), 4975-89.

[108] Thota, N.; Reddy, M. V.; Kumar, A.; Khan, I. A.; Sangwan, P. L.; Kalia, N. P.; Koul, J. L.; Koul, S., Substituted dihydronaphthalenes as efflux pump inhibitors of Staphylococcus aureus. Eur J Med Chem 2010, 45 (9), 360716.

[109] Fontaine, F.; Hequet, A.; Voisin-Chiret, A. S.; Bouillon, A.; Lesnard, A.; Cresteil, T.; Jolivalt, C.; Rault, S., First identification of boronic species as novel potential inhibitors of the Staphylococcus aureus NorA efflux pump. $J$ Med Chem 2014, 57 (6), 2536-48.

[110] Fontaine, F.; Hequet, A.; Voisin-Chiret, A. S.; Bouillon, A.; Lesnard, A.; Cresteil, T.; Jolivalt, C.; Rault, S., Boronic species as promising inhibitors of the Staphylococcus aureus NorA efflux pump: study of 6- substituted pyridine-3-boronic acid derivatives. Eur $J$ Med Chem 2015, 95, 185-98.

[111] Brincat, J. P.; Carosati, E.; Sabatini, S.; Manfroni, G.; Fravolini, A.; Raygada, J. L.; Patel, D.; Kaatz, G. W.; Cruciani, G., Discovery of novel inhibitors of the NorA multidrug transporter of Staphylococcus aureus. J Med Chem 2011, 54 (1), 354-65.

[112] Vidaillac, C.; Guillon, J.; Arpin, C.; Forfar-Bares, I.; Ba, B. B.; Grellet, J.; Moreau, S.; Caignard, D. H.; Jarry, C.; Quentin, C., Synthesis of omeprazole analogues and evaluation of these as potential inhibitors of the multidrug efflux pump NorA of Staphylococcus aureus. Antimicrob Agents Chemother 2007, 51 (3), 831-8.

[113] Dalton, W. S.; Grogan, T. M.; Meltzer, P. S.; Scheper, R. J.; Durie, B. G.; Taylor, C. W.; Miller, T. P.; Salmon, S. E., Drug-resistance in multiple myeloma and non-Hodgkin's lymphoma: detection of P-glycoprotein and potential circumvention by addition of verapamil to chemotherapy. $J$ Clin Oncol 1989, 7 (4), 415-24.

[114] Aeschlimann, J. R.; Dresser, L. D.; Kaatz, G. W.; Rybak, M. J., Effects of NorA inhibitors on in vitro antibacterial activities and postantibiotic effects of levofloxacin, ciprofloxacin, and norfloxacin in genetically related strains of Staphylococcus aureus. Antimicrob Agents Chemother 1999, 43 (2), 335-40.

[115] Ng, E. Y.; Trucksis, M.; Hooper, D. C., Quinolone resistance mediated by nor $A$ : physiologic characterization and relationship to $f l q B$, a quinolone resistance locus on the Staphylococcus aureus chromosome. Antimicrob Agents Chemother 1994, 38 (6), 1345-55.

[116] Vidaillac, C.; Guillon, J.; Moreau, S.; Arpin, C.; Lagardere, A.; Larrouture, S.; Dallemagne, P.; Caignard, D. H.; Quentin, C.; Jarry, C., Synthesis of new 4-[2(alkylamino) ethylthio]pyrrolo[1,2-a]quinoxaline and 5-[2(alkylamino) ethylthio]pyrrolo[1,2-a]thieno[3,2-e]pyrazine derivatives, as potential bacterial multidrug resistance pump inhibitors. J Enzyme Inhib Med Chem 2007, 22 (5), 620-31. [117] Mullin, S.; Mani, N.; Grossman, T. H., Inhibition of Antibiotic Efflux in Bacteria by the Novel Multidrug Resistance Inhibitors Biricodar (VX-710) and Timcodar (VX-853). Antimicrob Agents Chemother 2004, 48 (11), 4171-4176.

[118] Bharate, J. B.; Singh, S.; Wani, A.; Sharma, S.; Joshi, P.; Khan, I. A.; Kumar, A.; Vishwakarma, R. A.; Bharate, S. B., Discovery of 4-acetyl-3-(4-fluorophenyl)-1-(p-tolyl)-5methylpyrrole as a dual inhibitor of human P-glycoprotein and Staphylococcus aureus NorA efflux pump. Org Biomol Chem 2015, 13 (19), 5424-31.

[119] Leitner, I.; Nemeth, J.; Feurstein, T.; Abrahim, A.; Matzneller, P.; Lagler, H.; Erker, T.; Langer, O.; Zeitlinger, M., The third-generation P-glycoprotein inhibitor tariquidar may overcome bacterial multidrug resistance by increasing intracellular drug concentration. J Antimicrob Chemother 2011, 66 (4), 834-9.

[120] Kaatz, G. W.; Moudgal, V. V.; Seo, S. M.; Hansen, J. B.; Kristiansen, J. E., Phenylpiperidine selective serotonin reuptake inhibitors interfere with multidrug efflux pump 
activity in Staphylococcus aureus. Int J Antimicrob Agents 2003, 22 (3), 254-61.

[121] Wei, P.; Kaatz, G. W.; Kerns, R. J., Structural differences between paroxetine and femoxetine responsible for differential inhibition of Staphylococcus aureus efflux pumps. Bioorg Med Chem Lett 2004, 14 (12), 3093-7.

[122] German, N.; Kaatz, G. W.; Kerns, R. J., Synthesis and evaluation of PSSRI-based inhibitors of Staphylococcus aureus multidrug efflux pumps. Bioorg Med Chem Lett 2008, 18 (4), 1368-73.

[123] Kalle, A. M.; Rizvi, A., Inhibition of Bacterial Multidrug Resistance by Celecoxib, a Cyclooxygenase-2 Inhibitor. Antimicrob Agents Chemother 2011, 55 (1), 439442.

[124] Sabatini, S.; Gosetto, F.; Serritella, S.; Manfroni, G.; Tabarrini, O.; Iraci, N.; Brincat, J. P.; Carosati, E.; Villarini, M.; Kaatz, G. W.; Cecchetti, V., Pyrazolo[4,3c] [1,2]benzothiazines 5,5-dioxide: a promising new class of Staphylococcus aureus NorA efflux pump inhibitors. $J$ Med Chem 2012, 55 (7), 3568-72.

[125] Kaatz, G. W.; Moudgal, V. V.; Seo, S. M.; Kristiansen, J. E., Phenothiazines and thioxanthenes inhibit multidrug efflux pump activity in Staphylococcus aureus. Antimicrob Agents Chemother 2003, 47 (2), 719-26.

[126] Sabatini, S.; Kaatz, G. W.; Rossolini, G. M.; Brandini, D.; Fravolini, A., From phenothiazine to 3-phenyl-1,4benzothiazine derivatives as inhibitors of the Staphylococcus aureus NorA multidrug efflux pump. J Med Chem 2008, 51 (14), 4321-30.

[127] Kristiansen, M. M.; Leandro, C.; Ordway, D.; Martins, M.; Viveiros, M.; Pacheco, T.; Molnar, J.; Kristiansen, J. E.; Amaral, L., Thioridazine reduces resistance of methicillinresistant Staphylococcus aureus by inhibiting a reserpinesensitive efflux pump. In Vivo 2006, 20 (3), 361-6.

[128] Kristiansen, J. E.; Hendricks, O.; Delvin, T.; Butterworth, T. S.; Aagaard, L.; Christensen, J. B.; Flores, V. C.; Keyzer, H., Reversal of resistance in microorganisms by help of non-antibiotics. J Antimicrob Chemother 2007, 59 (6), 1271-9.

[129] Alnaseri, H.; Arsic, B.; Schneider, J. E.; Kaiser, J. C.; Scinocca, Z. C.; Heinrichs, D. E.; McGavin, M. J., Inducible Expression of a Resistance-Nodulation-Division-Type Efflux Pump in Staphylococcus aureus Provides Resistance to Linoleic and Arachidonic Acids. J Bacteriol 2015, 197 (11), 1893-905.

[130] Schindler, B. D.; Frempong-Manso, E.; DeMarco, C. E.; Kosmidis, C.; Matta, V.; Seo, S. M.; Kaatz, G. W., Analyses of multidrug efflux pump-like proteins encoded on the Staphylococcus aureus chromosome. Antimicrob Agents Chemother 2015, 59 (1), 747-8.

[131] Murakami, S.; Nakashima, R.; Yamashita, E.; Yamaguchi, A., Crystal structure of bacterial multidrug efflux transporter AcrB. Nature 2002, 419 (6907), 587-93.

[132] Kim, J.-S.; Jeong, H.; Song, S.; Kim, H.-Y.; Lee, K.; Hyun, J.; Ha, N.-C., Structure of the Tripartite Multidrug Efflux Pump AcrAB-TolC Suggests an Alternative Assembly Mode. Mol Cells 2015, 38 (2), 180-186.
[133] Du, D.; Wang, Z.; James, N. R.; Voss, J. E.; Klimont, E.; Ohene-Agyei, T.; Venter, H.; Chiu, W.; Luisi, B. F., Structure of the AcrAB-TolC multidrug efflux pump. Nature 2014, 509 (7501), 512-515.

[134] Pan, Y. P.; Xu, Y. H.; Wang, Z. X.; Fang, Y. P.; Shen, J. L., Overexpression of MexAB-OprM efflux pump in carbapenem-resistant Pseudomonas aeruginosa. Arch Microbiol 2016, 198 (6), 565-71.

[135] Lorenzi, V.; Muselli, A.; Bernardini, A. F.; Berti, L.; Pages, J. M.; Amaral, L.; Bolla, J. M., Geraniol restores antibiotic activities against multidrug-resistant isolates from Gram-negative species. Antimicrob Agents Chemother 2009, 53 (5), 2209-11.

[136] Aparna, V.; Dineshkumar, K.; Mohanalakshmi, N.; Velmurugan, D.; Hopper, W., Identification of Natural Compound Inhibitors for Multidrug Efflux Pumps of Escherichia coli and Pseudomonas aeruginosa Using In Silico High-Throughput Virtual Screening and In Vitro Validation. PLOS ONE 2014, 9 (7), e101840.

[137] Ohene-Agyei, T.; Mowla, R.; Rahman, T.; Venter, H., Phytochemicals increase the antibacterial activity of antibiotics by acting on a drug efflux pump. Microbiologyopen 2014, 3 (6), 885-96.

[138] Negi, N.; Prakash, P.; Gupta, M. L.; Mohapatra, T. M., Possible Role of Curcumin as an Efflux Pump Inhibitor in Multi Drug Resistant Clinical Isolates of Pseudomonas aeruginosa. J Clin Diagn Res 2014, 8 (10), DC04-7.

[139] Lee, M. D.; Galazzo, J. L.; Staley, A. L.; Lee, J. C.; Warren, M. S.; Fuernkranz, H.; Chamberland, S.; Lomovskaya, O.; Miller, G. H., Microbial fermentationderived inhibitors of efflux-pump-mediated drug resistance. Farmaco 2001, 56 (1-2), 81-5.

[140] Nguyen, S. T.; Kwasny, S. M.; Ding, X.; Cardinale, S. C.; McCarthy, C. T.; Kim, H. S.; Nikaido, H.; Peet, N. P.; Williams, J. D.; Bowlin, T. L.; Opperman, T. J., Structureactivity relationships of a novel pyranopyridine series of Gram-negative bacterial efflux pump inhibitors. Bioorg Med Chem 2015, 23 (9), 2024-34.

[141] Opperman, T. J.; Kwasny, S. M.; Kim, H. S.; Nguyen, S. T.; Houseweart, C.; D'Souza, S.; Walker, G. C.; Peet, N. P.; Nikaido, H.; Bowlin, T. L., Characterization of a novel pyranopyridine inhibitor of the AcrAB efflux pump of Escherichia coli. Antimicrob Agents Chemother 2014, 58 (2), 722-33.

[142] Renau, T. E.; Leger, R.; Flamme, E. M.; Sangalang, J.; She, M. W.; Yen, R.; Gannon, C. L.; Griffith, D.; Chamberland, S.; Lomovskaya, O.; Hecker, S. J.; Lee, V. J.; Ohta, T.; Nakayama, K., Inhibitors of efflux pumps in Pseudomonas aeruginosa potentiate the activity of the fluoroquinolone antibacterial levofloxacin. J Med Chem 1999, 42 (24), 4928-31.

[143] Lomovskaya, O.; Warren, M. S.; Lee, A.; Galazzo, J.; Fronko, R.; Lee, M.; Blais, J.; Cho, D.; Chamberland, S.; Renau, T.; Leger, R.; Hecker, S.; Watkins, W.; Hoshino, K.; Ishida, H.; Lee, V. J., Identification and characterization of inhibitors of multidrug resistance efflux pumps in Pseudomonas aeruginosa: novel agents for combination therapy. Antimicrob Agents Chemother 2001, 45 (1), 105-16. 
[144] Renau, T. E.; Leger, R.; Flamme, E. M.; She, M. W.; Gannon, C. L.; Mathias, K. M.; Lomovskaya, O.; Chamberland, S.; Lee, V. J.; Ohta, T.; Nakayama, K.; Ishida, Y., Addressing the stability of C-capped dipeptide efflux pump inhibitors that potentiate the activity of levofloxacin in Pseudomonas aeruginosa. Bioorg Med Chem Lett 2001, 11 (5), 663-7.

[145] Renau, T. E.; Leger, R.; Filonova, L.; Flamme, E. M.; Wang, M.; Yen, R.; Madsen, D.; Griffith, D.; Chamberland, S.; Dudley, M. N.; Lee, V. J.; Lomovskaya, O.; Watkins, W. J.; Ohta, T.; Nakayama, K.; Ishida, Y., Conformationallyrestricted analogues of efflux pump inhibitors that potentiate the activity of levofloxacin in Pseudomonas aeruginosa. Bioorg Med Chem Lett 2003, 13 (16), 2755-8.

[146] Renau, T. E.; Leger, R.; Yen, R.; She, M. W.; Flamme, E. M.; Sangalang, J.; Gannon, C. L.; Chamberland, S.; Lomovskaya, O.; Lee, V. J., Peptidomimetics of efflux pump inhibitors potentiate the activity of levofloxacin in Pseudomonas aeruginosa. Bioorg Med Chem Lett 2002, 12 (5), 763-6.

[147] Vargiu, A. V.; Ruggerone, P.; Opperman, T. J.; Nguyen, S. T.; Nikaido, H., Molecular mechanism of MBX2319 inhibition of Escherichia coli AcrB multidrug efflux pump and comparison with other inhibitors. Antimicrob Agents Chemother 2014, 58 (10), 6224-34.

[148] Sjuts, H.; Vargiu, A. V.; Kwasny, S. M.; Nguyen, S. T.; Kim, H. S.; Ding, X.; Ornik, A. R.; Ruggerone, P.; Bowlin, T. L.; Nikaido, H.; Pos, K. M.; Opperman, T. J., Molecular basis for inhibition of AcrB multidrug efflux pump by novel and powerful pyranopyridine derivatives. Proc Natl Acad Sci U S A 2016, 113 (13), 3509-14.

[149] Bohnert, J. A.; Kern, W. V., Selected arylpiperazines are capable of reversing multidrug resistance in Escherichia coli overexpressing RND efflux pumps. Antimicrob Agents Chemother 2005, 49 (2), 849-52.

[150] Kern, W. V.; Steinke, P.; Schumacher, A.; Schuster, S.; von Baum, H.; Bohnert, J. A., Effect of 1-(1naphthylmethyl)-piperazine, a novel putative efflux pump inhibitor, on antimicrobial drug susceptibility in clinical isolates of Escherichia coli. J Antimicrob Chemother 2006, 57 (2), 339-43.

[151] Opperman, T. J.; Nguyen, S. T., Recent advances toward a molecular mechanism of efflux pump inhibition. Front Microbiol 2015, 6, 421.

[152] Nakayama, K.; Ishida, Y.; Ohtsuka, M.; Kawato, H.; Yoshida, K.-i.; Yokomizo, Y.; Hosono, S.; Ohta, T.; Hoshino, K.; Ishida, H.; Yoshida, K.; Renau, T. E.; Léger, R.; Zhang, J. Z.; Lee, V. J.; Watkins, W. J., MexAB-OprMSpecific efflux pump inhibitors in Pseudomonas aeruginosa. Part 1: Discovery and early strategies for lead optimization. Bioorg Med Chem Lett 2003, 13 (23), 4201-4204.

[153] Nakayama, K.; Ishida, Y.; Ohtsuka, M.; Kawato, H.; Yoshida, K.; Yokomizo, Y.; Ohta, T.; Hoshino, K.; Otani, T.; Kurosaka, Y.; Yoshida, K.; Ishida, H.; Lee, V. J.; Renau, T. E.; Watkins, W. J., MexAB-OprM specific efflux pump inhibitors in Pseudomonas aeruginosa. Part 2: achieving activity in vivo through the use of alternative scaffolds. Bioorg Med Chem Lett 2003, 13 (23), 4205-8.
[154] Nakayama, K.; Kawato, H.; Watanabe, J.; Ohtsuka, M.; Yoshida, K.; Yokomizo, Y.; Sakamoto, A.; Kuru, N.; Ohta, T.; Hoshino, K.; Yoshida, K.; Ishida, H.; Cho, A.; Palme, M. H.; Zhang, J. Z.; Lee, V. J.; Watkins, W. J., MexAB-OprM specific efflux pump inhibitors in Pseudomonas aeruginosa. Part 3: Optimization of potency in the pyridopyrimidine series through the application of a pharmacophore model. Bioorg Med Chem Lett 2004, 14 (2), 475-9.

[155] Nakayama, K.; Kuru, N.; Ohtsuka, M.; Yokomizo, Y.; Sakamoto, A.; Kawato, H.; Yoshida, K.; Ohta, T.; Hoshino, K.; Akimoto, K.; Itoh, J.; Ishida, H.; Cho, A.; Palme, M. H.; Zhang, J. Z.; Lee, V. J.; Watkins, W. J., MexAB-OprM specific efflux pump inhibitors in Pseudomonas aeruginosa. Part 4: Addressing the problem of poor stability due to photoisomerization of an acrylic acid moiety. Bioorg Med Chem Lett 2004, 14 (10), 2493-7.

[156] Yoshida, K.; Nakayama, K.; Kuru, N.; Kobayashi, S.; Ohtsuka, M.; Takemura, M.; Hoshino, K.; Kanda, H.; Zhang, J. Z.; Lee, V. J.; Watkins, W. J., MexAB-OprM specific efflux pump inhibitors in Pseudomonas aeruginosa. Part 5: Carbon-substituted analogues at the $\mathrm{C}-2$ position. Bioorg Med Chem 2006, 14 (6), 1993-2004.

[157] Yoshida, K.; Nakayama, K.; Yokomizo, Y.; Ohtsuka, M.; Takemura, M.; Hoshino, K.; Kanda, H.; Namba, K.; Nitanai, H.; Zhang, J. Z.; Lee, V. J.; Watkins, W. J., MexAB-OprM specific efflux pump inhibitors in Pseudomonas aeruginosa. Part 6: exploration of aromatic substituents. Bioorg Med Chem 2006, 14 (24), 8506-18.

[158] Yoshida, K.; Nakayama, K.; Ohtsuka, M.; Kuru, N.; Yokomizo, Y.; Sakamoto, A.; Takemura, M.; Hoshino, K.; Kanda, H.; Nitanai, H.; Namba, K.; Yoshida, K.; Imamura, Y.; Zhang, J. Z.; Lee, V. J.; Watkins, W. J., MexAB-OprM specific efflux pump inhibitors in Pseudomonas aeruginosa. Part 7: highly soluble and in vivo active quaternary ammonium analogue D13-9001, a potential preclinical candidate. Bioorg Med Chem 2007, 15 (22), 7087-97.

[159] Mallea, M.; Mahamoud, A.; Chevalier, J.; AlibertFranco, S.; Brouant, P.; Barbe, J.; Pages, J. M., Alkylaminoquinolines inhibit the bacterial antibiotic efflux pump in multidrug-resistant clinical isolates. Biochem $J$ 2003, 376 (Pt 3), 801-5.

[160] Chevalier, J.; Bredin, J.; Mahamoud, A.; Mallea, M.; Barbe, J.; Pages, J. M., Inhibitors of antibiotic efflux in resistant Enterobacter aerogenes and Klebsiella pneumoniae strains. Antimicrob Agents Chemother 2004, 48 (3), 1043-6.

[161] Ghisalberti, D.; Mahamoud, A.; Chevalier, J.; Baitiche, M.; Martino, M.; Pages, J. M.; Barbe, J., Chloroquinolines block antibiotic efflux pumps in antibiotic-resistant Enterobacter aerogenes isolates. Int $J$ Antimicrob Agents 2006, 27 (6), 565-9.

[162] Mahamoud, A.; Chevalier, J.; Baitiche, M.; Adam, E.; Pages, J. M., An alkylaminoquinazoline restores antibiotic activity in Gram-negative resistant isolates. Microbiology 2011, 157 (Pt 2), 566-71.

[163] Yilmaz, S.; Altinkanat-Gelmez, G.; Bolelli, K.; Guneser-Merdan, D.; Over-Hasdemir, M. U.; Yildiz, I.; AkiYalcin, E.; Yalcin, I., Pharmacophore generation of 2- 
substituted benzothiazoles as AdeABC efflux pump inhibitors in A. baumannii. SAR QSAR Environ Res 2014, 25 (7), 551-63.

[164] Yilmaz, S.; Altinkanat-Gelmez, G.; Bolelli, K.; Guneser-Merdan, D.; Ufuk Over-Hasdemir, M.; Aki-Yalcin, E.; Yalcin, I., Binding site feature description of 2substituted benzothiazoles as potential AcrAB-TolC efflux pump inhibitors in E. coli. SAR QSAR Environ Res 2015, 26 (10), 853-71.

[165] Zeng, B.; Wang, H.; Zou, L.; Zhang, A.; Yang, X.; Guan, Z., Evaluation and target validation of indole derivatives as inhibitors of the AcrAB-TolC efflux pump. Biosci Biotechnol Biochem 2010, 74 (11), 2237-41.

[166] Bohnert, J. A.; Szymaniak-Vits, M.; Schuster, S.; Kern, W. V., Efflux inhibition by selective serotonin reuptake inhibitors in Escherichia coli. J Antimicrob Chemother 2011, 66 (9), 2057-60.

[167] Piddock, L. J.; Garvey, M. I.; Rahman, M. M.; Gibbons, S., Natural and synthetic compounds such as trimethoprim behave as inhibitors of efflux in Gram-negative bacteria. J Antimicrob Chemother 2010, 65 (6), 1215-23.

[168] Li, B.; Yao, Q.; Pan, X. C.; Wang, N.; Zhang, R.; Li, J.; Ding, G.; Liu, X.; Wu, C.; Ran, D.; Zheng, J.; Zhou, H., Artesunate enhances the antibacterial effect of $\beta$-lactam antibiotics against Escherichia coli by increasing antibiotic accumulation via inhibition of the multidrug efflux pump system AcrAB-TolC. J Antimicrob Chemother 2011, 66 (4), 769-77.

[169] Bay, D. C.; Rommens, K. L.; Turner, R. J., Small multidrug resistance proteins: A multidrug transporter family that continues to grow. Biochim Biophys Acta 2008, 1778 (9), 1814-1838.

[170] Chen, Y.-J.; Pornillos, O.; Lieu, S.; Ma, C.; Chen, A. P.; Chang, G., X-ray structure of EmrE supports dual topology model. Proc Natl Acad Sci 2007, 104 (48), 1899919004.

[171] Suriyanarayanan, B.; Sarojini Santhosh, R., Docking analysis insights quercetin can be a non-antibiotic adjuvant by inhibiting Mmr drug efflux pump in Mycobacterium sp. and its homologue EmrE in Escherichia coli. J Biomol Struct Dyn 2015, 33 (8), 1819-34.

[172] Malkhed, V.; Mustyala, K. K.; Potlapally, S. R.; Vuruputuri, U., Identification of novel leads applying in silico studies for Mycobacterium multidrug resistant (MMR) protein. J Biomol Struct Dyn 2014, 32 (12), 1889-906.

[173] van Veen, H. W., Structural biology: Last of the multidrug transporters. Nature 2010, 467 (7318), 926-927.

[174] Tsuda, M.; Terada, T.; Asaka, J.; Ueba, M.; Katsura, T.; Inui, K., Oppositely directed $\mathrm{H}+$ gradient functions as a driving force of rat $\mathrm{H}+$ /organic cation antiporter MATE1. Am J Physiol Renal Physiol 2007, 292 (2), F593-8.

[175] Terada, T.; Inui, K., Physiological and pharmacokinetic roles of $\mathrm{H}+$ /organic cation antiporters (MATE/SLC47A). Biochem Pharmacol 2008, 75 (9), 168996.

[176] Tanaka, Y.; Hipolito, C. J.; Maturana, A. D.; Ito, K.; Kuroda, T.; Higuchi, T.; Katoh, T.; Kato, H. E.; Hattori, M.; Kumazaki, K.; Tsukazaki, T.; Ishitani, R.; Suga, H.; Nureki,
O., Structural basis for the drug extrusion mechanism by a MATE multidrug transporter. Nature 2013, 496 (7444), 24751.

[177] Radchenko, M.; Symersky, J.; Nie, R.; Lu, M., Structural basis for the blockade of MATE multidrug efflux pumps. Nat Commun 2015, 6, 7995.

[178] Omote, H.; Hiasa, M.; Matsumoto, T.; Otsuka, M.; Moriyama, Y., The MATE proteins as fundamental transporters of metabolic and xenobiotic organic cations. Trends Pharmacol Sci 2006, 27 (11), 587-93.

[179] Moriyama, Y.; Hiasa, M.; Matsumoto, T.; Omote, H., Multidrug and toxic compound extrusion (MATE)-type proteins as anchor transporters for the excretion of metabolic waste products and xenobiotics. Xenobiotica 2008, 38 (7-8), 1107-18.

[180] Kuroda, T.; Tsuchiya, T., Multidrug efflux transporters in the MATE family. Biochim Biophys Acta 2009, 1794 (5), 763-8.

[181] Du, D.; van Veen, H. W.; Murakami, S.; Pos, K. M.; Luisi, B. F., Structure, mechanism and cooperation of bacterial multidrug transporters. Curr Opin Struct Biol 2015, 33, 76-91.

[182] Kesherwani, M.; Michael Gromiha, M.; Fukui, K.; Velmurugan, D., Identification of novel natural inhibitor for NorM - a multidrug and toxic compound extrusion transporter - an in silico molecular modeling and simulation studies. J Biomol Struct Dyn 2017, 35 (1), 58-77. 
Title page

\section{Medicinal Chemistry Updates on Bacterial Efflux Pump Modulators}

F. Durães - Fernando André Pereira Marques Durães

Laboratory of Organic and Pharmaceutical Chemistry, Department of Chemical Sciences, Faculty of Pharmacy, University of Porto, Rua Jorge Viterbo Ferreira, 228, 4050-313 Porto, Portugal.

CIIMAR/CIMAR - Interdisciplinary Centre of Marine and Environmental Research, University of Porto. Terminal de Cruzeiros do Porto de Leixões, Avenida General Norton de Matos P 4450-208 Matosinhos, Portugal.

M. M. Pinto - Madalena Maria Magalhães Pinto Laboratory of Organic and Pharmaceutical Chemistry, Department of Chemical Sciences, Faculty of Pharmacy, University of Porto, Rua Jorge Viterbo Ferreira, 228, 4050-313 Porto, Portugal.

CIIMAR/CIMAR - Interdisciplinary Centre of Marine and Environmental Research, University of Porto. Terminal de Cruzeiros do Porto de Leixões, Avenida General Norton de Matos P 4450-208 Matosinhos, Portugal.

E. Sousa (corresponding author) - Maria Emília Silva Pereira de Sousa Laboratory of Organic and Pharmaceutical Chemistry, Department of Chemical Sciences, Faculty of Pharmacy, University of Porto, Rua Jorge Viterbo Ferreira, 228, 4050-313 Porto, Portugal. tel: +351220428689, fax: +351226093390, esousa@,ff.up.pt

CIIMAR/CIMAR - Interdisciplinary Centre of Marine and Environmental Research, University of Porto. Terminal de Cruzeiros do Porto de Leixões, Avenida General Norton de Matos P 4450-208 Matosinhos, Portugal. 


\title{
Medicinal Chemistry Updates on Bacterial Efflux Pump Modulators
}

\author{
Fernando Durães ${ }^{\mathrm{a}}$, Madalena Pinto ${ }^{\mathrm{a}}$, Emília Sousa $^{* a}$ \\ ${ }^{a}$ Laboratory of Organic and Pharmaceutical Chemistry, Department of Chemical Sciences, Faculty of \\ Pharmacy, University of Porto, Rua Jorge Viterbo Ferreira, 228, 4050-313 Porto, Portugal and \\ CIIMAR/CIMAR - Interdisciplinary Centre of Marine and Environmental Research, University of Porto. \\ Terminal de Cruzeiros do Porto de Leixões, Avenida General Norton de Matos P 4450-208 Matosinhos, \\ Portugal. tel: +351220428689, fax: +351226093390, esousa@ff.up.pt
}

\begin{abstract}
Antibiotic resistance is one of the most pressing health issues of our days. It can arise due to a multiplicity of factors, such as target modification, decrease in the drug uptake, changes in the metabolic pathways and activation of efflux pumps. The overexpression of efflux pumps is responsible for the extrusion of drugs, making antibiotic therapy fail, as the quantity of intracellular antibiotic is not enough to provide the desired therapeutic effect.

Efflux pumps can be included in five families according to their composition, nature of substrates, energy source, and number of transmembrane spanning regions. The $\mathrm{ABC}$ superfamily are mainly found in Gram-positive bacteria, use ATP as an energy source, and only a limited number of $\mathrm{ABC}$ pumps confer multidrug resistance (MDR).

On the other hand, the MFS family, most present in Gram-positive bacteria, and the RND family, characteristic of Gramnegative bacteria, are the most associated with antibiotic resistance. A wide variety of inhibitors have been disclosed for both families, from either natural or synthetic sources, or even drugs that are currently in therapy for other diseases.

The other two families are the SMR, which are the smallest drug efflux proteins known, and the MATE family, whose pumps can also resort to the sodium gradient as an energy source.

In this review, it is intended to present a comprehensive review of the classes of efflux pump inhibitors from the various sources, highlighting their structure-activity relationships, which can be useful for medicinal chemists in the pursuit of novel efflux pump inhibitors.
\end{abstract}

Keywords: Antimicrobial resistance; efflux pump inhibitors; natural products; synthetic compounds; existing drugs; Structure-activity relationship.

\section{INTRODUCTION}

Bacteria were discovered the late $19^{\text {th }}$ century as infectious agents. It was, however, half a century later that antibiotics completely revolutionized medicine and became a turning point in human history. Unfortunately, the misruled use of these drugs led to the appearance of resistant strains, and to the concern of a return to the pre-antibiotic era, where the simplest, most treatable infections can become deadly [1-2]. Antibiotic resistance can arise due to multiple causes. The most common are the overuse and/or inappropriate prescribing, as well as extensive veterinary and agricultural use. These factors are especially alarming if it is taken into account the very few new antibiotics that were discovered in the past years [3]. Due to their genetic plasticity, bacteria can develop defense mechanisms in response to environmental threats. As far as antibiotics are concerned, bacteria use two major strategies to adapt to these drugs, which are mutations in genes and the acquisition of resistance genes through horizontal gene transfer. The first strategy can be manifested through modification of the target, decrease in the drug uptake, activation of efflux pumps to extrude the drug or changes in metabolic pathways. The latter is related to the acquisition of external genetic material through transformation, transduction, or conjugation [4-5]. Recently, vesicle-mediated resistance has also been regarded as a mechanism of resistance acquisition [6].

Efflux pumps are ubiquitous in bacteria, and can be encoded by genes present either in the bacterial chromosomes or in plasmids [7-8]. They concern five families, according to their composition, substrates, energy sources, and number of transmembrane spanning regions: the adenosine triphosphate (ATP)-binding cassette (ABC) superfamily, the resistancenodulation-division family (RND), the major facilitator superfamily (MFS), the small multidrug resistance (SMR) 
family, and the multidrug and toxic compound extrusion (MATE) family (Figure 1) [7-12].

*Address correspondence to this author at the Department of Organic and Pharmaceutical Chemistry, Faculty of Pharmacy, University of Porto, P.O. Box: 4050-313, Porto, Portugal; Tel/Fax: +351 220428 689, +351 226093 390; E-mail: esousa@ff.up.pt

Efflux pumps can be further divided into two major families, the primary transporters, which hydrolyze ATP as a source of energy, as is the case of the ABC family, and secondary transporters, which use the proton or sodium gradient as a source of energy [13]. Overexpression of multidrug efflux pumps is correlated with an increase in the minimum inhibitory concentration (MIC) of antibiotics, and thus, these pumps can be considered potential antibacterial targets.

Therefore, the development of efflux pump inhibitors may be a way of fighting against resistant microorganisms. Studies have been carried out on the structure of efflux pumps, as well as on their mechanisms of binding and extrusion, leading to the possibility of structure-based design of efflux pump inhibitors (EPIs). The rationale for the development of these EPIs could be, for instance, the inhibition of the energy sources required for the ability to extrude antibiotics, and the development of compounds that compete with the antibiotics for their extrusion. However, the main challenge lies in the fact that many compounds that present EPI activity and are already used for the treatment of other diseases are toxic for human cells in the concentrations required to present EPI activity and, therefore, clinically useless [7-9, 13].

Even though there are already several reviews which describe different classes of EPIs [11, 13-31], most focus on a particular source of compounds or on a class of pumps or bacteria. Herein, it is intended to present a comprehensive review of the several classes of EPIs from natural and synthetic sources and from existing drugs in therapeutics and to highlight structure-activity relationship studies in the referred efflux pumps (ABC, RND, MFS, SMR and MATE), which can be useful for medicinal chemists in the pursuit of novel compounds for this goal.

\section{2. $\mathbf{A B C}$}

The $\mathrm{ABC}$ transporters are primary transporters, and they exist in every membrane systems, whether the organism is prokaryotic or eukaryotic. P-Glycoprotein (P-gp) is a very well known human $\mathrm{ABC}$ transporter, involved in the resistance of cytotoxic drugs used in cancer therapy [32-34], whose modulation has been regarded as a study field [3537]. Structurally, an ABC transporter consists of four conserved domains, two transmembrane domains, and two cytoplasmic domains, which bind to nucleotides and, therefore, are involved in ATP binding $[32,38]$. In fact, the nucleotide binding domains are responsible for hydrolyzing ATP, which will lead to conformational changes in the attached transmembrane domains, making it possible for substrates to cross the lipid bilayer of the membrane, either in an inwards or an outwards fashion [39].

The bacterial transporters are highly selective for sugars, amino acids, metallic cations, organo-iron complexes, vitamins, and antibiotics [32, 40]. They are found in Grampositive bacteria, such as Enterococcus faecalis and Staphylococcus aureus, and confer resistance to macrolides and bacitracin. However, only a limited number of $\mathrm{ABC}$ pumps are associated with MDR, which means that $\mathrm{ABC}$ pumps do not play a pivotal role in conferring antibiotic resistance in bacteria [32-33].

As such, bacterial ABC transporter inhibitors are scarce. The few EPIs discovered in this context are mainly against MsrA (Figure 2, PDB 1NWA), whose tridimensional structure has been elucidated [41].

The structures of the $\mathrm{ABC}$ inhibitors that will be further detailed throughout this section are comprised in Figure 3.

\subsection{ABC inhibitors}

\subsubsection{Natural Products}

The search for natural products comes from the reason that plants produce antimicrobials to protect themselves from exogenous organisms. It is thought that plants can produce anti-MDR compounds to fight the microbial resistance phenomenon. Carnosic acid (1) was one of the major products isolated from the plant Rosmarinus officinalis. When tested against the macrolide resistant strain of $S$. aureus, expressing the MsrA pump, compound 1 displayed favorable results, showing an impressive 8-fold potentiation of erythromycin [42].

An extract of Lycopus europaeus led to the isolation of two new isopimarane diterpenes, methyl-1 $\alpha$-acetoxy- $7 \alpha, 14 \alpha$ dihydroxy-8,15-isopimarandien-18-oate (2) and methyl$1 \alpha, 14 \alpha$ - diacetoxy-7 $\alpha$-hydroxy-8,15-isopimarandien-18-oate (3), which showed no antibacterial activity, but were able to reduce erythromycin's MIC from $256 \mu \mathrm{g} / \mathrm{ml}$ to $128 \mu \mathrm{g} / \mathrm{ml}$, therefore causing a 2 -fold potentiation. This behavior was similar to other isopimarane diterpenes isolated in this study, as well as for 5,9-dihydroxygeranyllinalool (4) and 9acetoxy-5-hydroxygeranyllinalool (5), two diterpenoids [4344].

Phytol (6) is an acyclic isoprenoid, and its EPI activity against the ABC pumps of $E$. coli was assessed in combination with nalidixic acid. Additionally, 15 derivatives were synthesized. One derivative in particular, compound 7 , was able to decrease the MIC of nalidixic acid by 16-fold, and four other derivatives reduced the MIC by 8 -fold. This effect was caused by the inhibition of ATP-dependent efflux pump and down-regulation of the efflux gene. In silico absorption, distribution, metabolism, and excretion (ADME) analysis was performed, taking into account parameters such as solubility in water, human intestinal absorption, plasma protein binding, blood-brain barrier penetration, hepatotoxicity and cytochrome P450 (CYP450) inhibition. Results showed all five active derivatives presented low 
permeability into the central nervous system and displayed moderate intestinal absorption. In terms of plasma protein binding level, all five compounds exhibited levels below $90 \%$, suggesting favorable distribution of the compounds into the blood. They also did not present hepatotoxicity, unlike reserpine, making them good lead compounds [45].

Ursolic acid (8) is a pentacyclic triterpenoid isolated from diverse natural sources. Its wide activity includes antiinflammatory, antimicrobial, diuretic, and antitumor activity, among others. When tested against Escherichia coli strains that were resistant and susceptible to nalidixic acid, ursolic acid did not display intrinsic antibacterial activity. However, ursolic acid (8) was able to reduce the MIC of nalidixic acid by 2 -fold, when both drugs were used in combination. This synergy led to further research on this scaffold. The first derivative prepared was the 3-O-acetyl ursolic acid, and showed a 4-fold decrease in the MIC of nalidixic acid. The lipophilic nature of this derivative, allied with the increased potency, led to the synthesis of other lipophilic ester derivatives. The methyl and ethyl esters of 3-O-acetyl ursolic acid did not show any increment in the activity. However, a further increase in the ester carbon chain increased the drug reversal potential by 2 -fold, in comparison to 3-O-acetyl ursolic acid, against the resistant strain. This is the case of the isopropyl (9) and the $n$-butyl (10) derivatives. The addition of a pentyl group led to a decrease in activity. Furthermore, ursolic acid (8), and derivatives 9 and $\mathbf{1 0}$, reduced the MIC of tetracycline by 2 -fold, 8 -fold, and 8 -fold, respectively. When these compounds were tested against ATP-dependent efflux pumps, it was found that derivatives 9 and 10 were successful in inhibiting these pumps in terms of the liberated inorganic phosphate. Molecular modeling studies with the YojI pump, using maltose/maltodextrin transport ATP-binding protein as a homology model, showed that 9 was bound to the nucleophilic Ser-78, to the aromatic Phe-81, Trp-85 and Leu-86, the acidic Glu-94 and Glu-144 and the basic Ala-79, Ala-139, Ala-140, Ala-141, Ala-143, Arg-74, Arg-122, Arg-146, Leu-107, Val-80, Lys-96, and Ile-123 in the nucleotide binding domain. Derivative 10 was also subjected to the same kind of studies using the same model, and was predicted to bind in the same region [46].

A study with flavones and isoflavones from the plant Sophora moorcroftiana revealed that diosmetin (11) was able to inhibit three drug resistant strains of $S$. aureus, including the $\mathrm{ABC}$ family MsrA overexpressing strain, potentiating the effects of norfloxacin and streptomycin [47]. These results corroborate the works of Chan et al (2013), who had previously studied the effects of diosmetin (11) as an EPI [48]. This group observed a synergistic effect of this flavonoid with erythromycin, which was 5-fold greater than that of verapamil [48].

4-Hydroxy- $\alpha$-tetralone (12) isolated from the plant Ammannia multiflora, showed enhancement against nalidixic acid resistant strains of Escherichia coli, prompting the synthesis of derivatives in order to infer their mechanism of action. Five derivatives were synthesized, and it was shown that compound $\mathbf{1 3}$ was able to inhibit the expression of the $\mathrm{ABC}$ transporter protein gene yojI, which encodes the ABC protein YojI, and was successful in combination with tetracycline [49]. Molecular modelling studies with compound 13 and a homology model of the YojI protein revealed the following bound residues: Ser-78 (nucleophilic), Leu-86, Ile-123, Ala-137, Ala-140, Ala-141, and Ala-143 (hydrophobic), Glu-144 (acidic), Arg-122 (basic), Phe-81 (aromatic), Glu-144 (acidic), Arg-146 and Lys-96 (basic), and Trp-85 (hydrophobic), the same region as noted for ursolic acid derivatives 9 and 10. Compound 13 showed good affinity and strong hydrophobic interaction with the YojI protein, corroborating the fact that this compound acts by the inhibition of ATP dependent efflux pumps [49].

The plant Portulaca oleraceae is used in folk medicine for its anti-inflammatory, anti-septic and diuretic effects, among others. A study of its fatty acids demonstrated that linoleic (14) and oleic acids (15) had synergistic activity with macrolides for the MsrA overexpressing strain of methicillin-resistant $S$. aureus (MRSA). In fact, erythromycin's activity was enhanced when in combination with either of these two compounds, likely through the inhibition of the MsrA ATP dependent pump. Since these fatty acids are essential for humans, low toxicity is expected, making them promising agents to use in combination with antibiotics [50].

Lysergol (16) is a clavine alkaloid of the ergoline family. Recently, its EPI activity was studied, and derivatives were synthesized [51]. The compounds were tested against susceptible and resistant to nalidixic acid strains of $E$. coli and a tetracycline resistant strain. While not possessing antibacterial activity, lysergol (16) showed synergy with nalidixic acid at the concentration of $10 \mu \mathrm{g} / \mathrm{ml}$, reducing its MIC by 8 -fold in the resistant strain of $E$. coli and 4-fold in the susceptible strain. Of the thirteen semi-synthetic derivatives prepared, derivatives $\mathbf{1 7}$ and $\mathbf{1 8}$ reduced nalidixic acid's MIC by 8 -fold, twice as much as lysergol (16), in the susceptible strain. A 4-fold potentiation of nalidixic acid was observed for the three compounds when tested against the resistant strain. As for the tetracycline resistant strain, all three compounds were able to reduce tetracycline's MIC by 8 -fold. Further studies were performed, in order to conclude whether or not these compounds were capable of interfering with ATP-dependent efflux pumps, and it was proven that all three compounds inhibited ATPase activity, concerning the liberation of inorganic phosphate. A down-regulation of the gene yojI was also observed when the tetracycline resistant strain was treated with lysergol (16) and its two most potent derivatives, 17 and 18, which further confirms the activity of these compounds as $\mathrm{ABC}$ transporter inhibitors [51].

\subsubsection{Existing Drugs}

As part of a screening program for P-gp inhibitors, GG918 (19) was discovered. This synthetic compound had already showed success in increasing the intracellular concentration of paclitaxel in cancer cells. In $S$. aureus, this compound was able to slightly potentiate the activity of norfloxacin in a strain overexpressing the ABC efflux pump 
MsrA, suggesting that it may possess minimal activity over this pump [52].

The human P-gp inhibitors, vanadates, have been studied as inhibitors of the MutS pumps, belonging to the ABC ATPase superfamily. In fact, these compounds have demonstrated activity against the MutS in $P$. aeruginos $a$ and $E$. coli. Decavanadate was the most effective, followed by orthovanadate (20), and they act non-competitively, possibly by replacing the inorganic phosphate. The fact that the $\mathrm{ABC}$ ATPases inhibited by orthovanadate are phosphorylated leads to the assumption that the low inhibitory effect could be attributed to the absence of a stable phosphorylation state of MutS [53].

\section{MFS}

The MFS is the most extensively studied family of transporters, present in both Gram-positive and Gramnegative bacteria, and comprises uniporters, symporters and antiporters. It also constitutes the largest family of secondary transporters, with over 10000 sequenced members. Its substrates are diverse, and can range from ions, to carbohydrates, lipids, amino acids, nucleosides, among others. As many members of the MFS originate bacterial MDR, these transporters can pose as a good system for modulation studies [7, 19, 38, 54].

With the elucidation of the crystal structure of the MFS pump EmrD of E. coli (Figure 4, PDB 2GFP) [55], a general structure could be proposed for MFS transporters: twelve transmembrane helices that form a compact structure, with four of these transmembrane helices facing away from the interior cavity and the rest forming the internal cavity, constituted mostly by hydrophobic residues [7].

The efflux of drugs is only possible if the carrier alternates between two conformations, so that the substrate can bind to the convenient site in either side of the membrane. However, a limitation concerning the energy involved to displace the exposed hydrophilic surface of a protein can be inferred. It has been proved that either the oligomerization domain or the transport subunit provide the support needed to the movement of the substrate binding site, through a rigid-body rotation of the substrate binding domain, referred to as the "elevator mechanism" [56-57]. For this family, the most extensively studied efflux pump is the NorA pump of $S$. aureus.

The inhibitors that have proven activity against efflux pumps of the MFS family are gathered in Figure 5.

\subsection{MFS inhibitors}

\subsubsection{Natural Products and Derivatives}

Terpenes and terpenoids, existent in the essential oils of plants, have showed promising therapeutic potential in various fields. Therefore, studies about EPI potential of these compounds were carried out. Monoterpenes nerol (21), estragole (22), and dimethyl-octanol (23) were tested against
S. aureus. Compounds 21 and $\mathbf{2 3}$ were able to enhance the activity of norfloxacin, decreasing its MIC against the NorA overexpressing strain while not displaying intrinsic antibacterial activity. These open chain terpenes proved to be more effective than 22, which carried a benzene ring, at inhibiting the NorA pumps [58].

Citronellal (24) and citral (25) are monoterpenes, and were chosen as starting material for the synthesis of new amide derivatives. The basis for this study was the fact that aromatic amides are recognized as potent EPIs, whereas alkyl, alkenyl, and alkynil amides had not yet been explored. The compounds obtained were 5,9-dimethyldeca-2,4,8trienoic acid amides and 5,9-dimethyldeca-2,8-dienoic acid amides, whose activity for the potentiation of ciprofloxacin in $S$. aureus was assessed. Of the first 22 synthesized compounds, seven were able to reduce ciprofloxacin's MIC by 4 -fold. Further modifications were performed, and the $\mathrm{C}$ 9 methyl group was replaced by an amide, yielding compounds with similar potency as the previous ones. Derivatives subjected to hydrogenation led to decreased activity, which reveals that unsaturation is an important feature for antimicrobial potentiation. The mechanism of inhibition was assessed through ethidium bromide efflux, confirming these compounds act by inhibiting NorA, being compound 26, a 9-formyl-5-methyldeca-2,4,8-trienoic acid amide, the most potent inhibitor [59].

Ferruginol (27), a diterpene, showed efficiency in inhibiting the efflux of ethidium bromide, proving its activity as a NorA inhibitor. It also showed a decrease in norfloxacin efflux in S. aureus [60]. Totarol (28), a phenolic diterpene, also showed a reduction in ethidium bromide efflux, suggesting activity against NorA pump, even though its mechanism is not yet known [61].

The triterpene ginsenoside $20(S)$ - Rh2 (29), a trace compound from red ginseng, also enhanced the effect of ciprofloxacin against $S$. aureus, through a NorA-mediated efflux inhibition, promoting the antibiotic accumulation in the bacteria [62].

Reserpine (30) is a natural alkaloid derived from Rauwolfia vomitoria, known as an anti-hypertensive agent, with inhibitory activity against human P-gp and breast cancer resistance protein (BCRP) [63-64]. Reserpine (30) has proven to be an effective inhibitor of the Bmr multidrug transporter in Bacillus subtilis, diminishing the efflux of ethidium bromide. Its structural analogue rescinnamine also had EPI activity, in a smaller extension than reserpine (30) [65]. Through DNA sequencing of the $b m r$ genes of mutants, it was shown that reserpine (30) interacts with the residues Phe-143, Val-286, and Phe-306 of Bmr pumps, inhibiting drug transport [19]. Compound $\mathbf{3 0}$ has shown potential as an EPI for the inhibition of NorA efflux pumps in S. aureus, which are structurally related to Bmr pumps, reversing susceptibility to fluoroquinolones, demonstrating reductions up to 4-fold in MIC for some tested strains. It has been shown that 30's effect is more pronounced in hydrophilic fluoroquinolones, such as ciprofloxacin [66]. The mechanism by which $\mathbf{3 0}$ performs the inhibitory activity is through direct binding to the transporters that mediate drug 
efflux, acting as a competitive inhibitor [19, 67]. Reserpine (30) also succeeded in inhibiting the chloramphenicol efflux by CmlR1 and CmlR2 of Streptomyces coelicolor, the ciprofloxacin and norfloxacin efflux in Listeria monocytogenes's pump Lde, and the kanamycin and fusidic acid efflux by LmrS from a MRSA clinical isolate. On the other hand, for L. monocytogenes, $\mathbf{3 0}$ was not able to reverse susceptibility to linezolid and lincomycin, which suggests that 30 does not completely overlap with the structure binding sites of efflux pumps, being the inhibition dependent on the type and nature of the substrate [19]. However, reserpine (30) shows neurotoxicity at the concentration required to inhibit NorA, leading to the need to investigate new inhibitors [67].

Piperine (31), an alkaloid found in the fruits of Piper nigrum, was found to be an inhibitor of ciprofloxacin efflux in $S$. aureus, reducing the $\mathrm{MIC}$ by 2 -fold. It is thought that compound $\mathbf{3 1}$ acts as a direct inhibitor of drug efflux. Compound 31 inhibits drug transport of the pump MdeA, potentiating the effects of benzalkonium chloride, doxorubicin, daunorubicin, novobiocin, tetraphenylphosphonium chloride, rhodamine 6G, virginiamycin and mupirocin [17, 19-20]; it also inhibits ciprofloxacin efflux by NorA [68].

Given these positive results obtained with piperine (31), further research into this scaffold was performed. Kumar et al. (2008) performed a screening of about 200 structurally diverse compounds for the inhibition of NorA efflux pump, choosing ethidium bromide as a substrate, since the only mechanism through which this compound is expelled is active transport (68). About 50 compounds were able to increase the intracellular concentration of ethidium bromide by 4 -fold, while not displaying antibacterial activity. Five of these compounds were more potent than piperine (31), being three of these compounds selected for further studies: 32, 33, and 34, being 32 and 34 more potent than 31 by 40 -fold [18, 68]. Assumptions regarding structure-activity relationship (SAR) studies of these molecules could be drawn (Figure 6). It was demonstrated that the introduction of an alkyl group at C-4 position, mainly an isopropyl, isobutyl or di-isopropyl group, coupled with the substitution of the piperidinyl moiety with an aromatic amine, such as aniline, would lead to maximum potentiation of ciprofloxacin; if the anilinyl moiety is substituted with a 2-hydroxymethyl group or a nitrile, regardless of the positional isomery, ciprofloxacin activity would increase. Replacing the piperidinyl moiety with a phenylacetamide yields good potentiation of ciprofloxacin, reducing its MIC by 4-fold, regardless of the isomery. Unsaturation is crucial for the potentiation, as well as the amide. Lastly, the retention of 3,4methylenedioxyphenyl or 4-methoxyphenyl groups in the 1,3-benzodiol moiety also gives favorable results [68-70]. Plants of the Berberis species have the ability to produce the alkaloid berberine, which is a plant secondary metabolite produced in response to microbial invasion, whose activity is enhanced by a flavolignane also produced by these species, 5'-methoxyhydnocarpin (35) [71]. Compound 35 exists in a mixture of anion and neutral compound at physiological $\mathrm{pH}$, since it has a phenolic group with a pKa of 7.3. Due to the acidic properties of this 7-OH group, 35 is likely to be a specific microbial MDR inhibitor, as flavonoids with P-gp inhibitor properties usually possess alkylated 7-O groups. In fact, when added to a wild type strain of $S$. aureus, compound 35 caused a decrease in norfloxacin's MIC to $0.25 \mu \mathrm{g} / \mathrm{ml}$. Furthermore, when tested with berberine and palmatine, 35 appeared to completely inhibit NorA, suggesting it acts as a non-competitive inhibitor [71].

A number of hydnocarpin-type flavolignanes were synthesized in order to perform SAR studies. Flavolignanes with and without phenolic groups at the 5 and 7 positions had comparable activity. Diosmetin (11), the parent compound, presents hydroxyl groups in the A ring and was the most potent compound. Peracetate derivatives of $\mathbf{3 5}$ have approximately the same potency as their parent compounds, and could be considered prodrugs, if deacetylated by $S$. aureus, which seems unlikely. Considering the D ring, compounds bearing a 3'"-methoxy-4"'-hydroxy moiety displayed good activity, and 3',5',-dimethoxy-4',-hydroxy moieties conferred a slightly less active compound. As for the $\mathrm{B}$ ring, the presence of an $\mathrm{H}$ or an $\mathrm{OH}$ is not critical for activity [72]. In addition, a flavone isolated from Alkanna orientalis, sarothrin (36), blocked ethidium bromide efflux by $S$. aureus, suggesting its potential activity as a NorA inhibitor [73].

Biochanin A (37), an isoflavone, also showed potentiation of norfloxacin and berberine against wild-type $S$. aureus and Bacillus megaterium. An analogue of luteolin, orobol (38), was quite active as a NorA inhibitor, unlike luteolin itself. It was shown that flavones which have a single methoxy in its B-ring are more potent than flavones with two methoxy groups in the B-ring [74].

Another flavone, baicalein (39), was capable of potentiating the effect of ciprofloxacin in $S$. aureus. Studies aimed at measuring the efflux of ciprofloxacin and pefloxacin, a poor NorA substrate, were performed, and showed that compound 39 was much more potent at restoring ciprofloxacin sensitivity than pefloxacin, leading to the conclusion that this flavone acts as a NorA efflux inhibitor, even though its mechanism remains unknown [75].

Maia et al. (2011) identified six flavonoids from the plant Praxelis clematidea: apigenine, genkwanin, 7,4'dimethylapigenin, trimethylapigenin, cirsmaritin, and tetramethylscutellarein (40) [76]. These methoxylated flavones showed no antibacterial activity, but were able to reduce the MIC of norfloxacin at least by 2 -fold and up to 16-fold, as was the case of $\mathbf{4 0}$, at a concentration of $8 \mu \mathrm{g} / \mathrm{ml}$, being therefore considered NorA efflux pump modulators. It is thought that the lipophilicity of the flavonoids, strengthened by the presence of methoxyl groups, is responsible for this activity [21]. Presence of a methoxy in the 4' position proved to be decisive for activity, as flavonoids containing this group were more potent. The number of methoxy groups is also essential for potency, being the most metoxylated compounds the most active [76]. Tiliroside (41), an amphipathic kaempferol glycoside, reduced the concentration of antibiotic needed to inhibit the 
growth of bacteria. The lipophilicity of the flavone moiety may be a determinant factor for the activity displayed. Tiliroside (41) showed no antibacterial activity against $S$. aureus, but showed a decrease in the MIC of fluoroquinolones up to 16-fold, when at a concentration of $64 \mu \mathrm{g} / \mathrm{ml}$. The reduction in the MIC of biocides, such as ethidium bromide and benzalkonium chloride, was impressive, with the MIC of acriflavine showing a decrease by 128 -fold at the concentration of $64 \mu \mathrm{g} / \mathrm{ml}$ and $32 \mu \mathrm{g} / \mathrm{ml}$. These results suggest that $\mathbf{4 1}$ might additionally act on pumps other than NorA [77].

Kaempferol 3-O- $\alpha$-L-(2, 4-bis- $E$ - $p$-coumaroyl)rhamnoside (42) was also able to potently inhibit in a concentration dependent manner NorA-mediated ethidium bromide efflux in $S$. aureus. This compound also increased the antibiotic effect of norfloxacin against a wild-type and a NorA overexpressing strain of $S$. aureus. However, it has to be considered that $\mathbf{4 1}$ and $\mathbf{4 2}$ are flavonoid glycosides and cinnamoyl esters, and that their activity can become compromised with the activity small intestine $\beta$-glucosidases or plasma esterases, even though the activity might possibly lie in the kaempferol moiety, with 41 and 42 acting as prodrugs. The authors hypothesized that if the activity of compound $\mathbf{4 2}$ is compromised, this compound could be used as adjuvant of ciprofloxacin for topical infections [78].

Since 42 showed promising results as a NorA EPI, the same group performed a screening of a library of 117 chalcones, due to their structural similarities with the coumaroyl substituents [79]. Ten out of the 117 screened chalcones presented medium efflux inhibition at $20 \mu \mathrm{g} / \mathrm{ml}$. The most potent chalcones, such as $\mathbf{4 3}$, bear a dimethylaminoethoxy moiety, which might disrupt the proton gradient, thus eliminating the energy source of NorA. Five of these chalcones possessed a hydroxyl group at the 4'-position, and potentiated the activity of berberine against $S$. aureus and $B$. cereus, suggesting that this substituent is also important for activity. The compound with a methoxy group at position $\mathrm{C}$ 2 has shown to present the best activity. The majority of chalcones tested showed a 4-fold reduction of ciprofloxacin's MIC in $S$. aureus, suggesting the presence of a NorA efflux pump inhibitory activity. Compound 43 showed 8-fold and 16-fold reduction of the MIC of ciprofloxacin at 6.25 and $12.5 \mu \mathrm{g} / \mathrm{ml}$, respectively. However, it also showed antibacterial activity at $25 \mu \mathrm{g} / \mathrm{ml}$. Some chalcones also presented antiparasitic activity, namely against Leishmania major and Plasmodium yoelii [79].

Another chalcone, 4',6'-dihydroxy-3',5'-dimethyl-2'methoxychalcone (44), showed good activity as a MDR, causing complete growth inhibition at a very low concentration $(3.3 \mu \mathrm{g} / \mathrm{ml})$ when combined with a sub inhibitory concentration of berberine. When tested in combination with berberine, erythromycin, and tetracycline against strains of $S$. aureus, there was an increase in the activity of all tested compounds in the wild-type $S$. aureus, indicating a mode of action likely related to the NorA pump. This compound was also tested against $B$. cereus, potentiating the effects of the tested antibiotics as well, and displaying a favorable effect, particularly in the case of berberine, where a 30-fold increase in the activity was observed [80].

Pheophorbide A (45) is an intermediate of the natural breakdown of chlorophyll. Concentrations as low as 0.5 $\mu \mathrm{g} / \mathrm{ml}$ of pheophorbide A with sub-inhibitory concentrations of berberine were enough to completely inhibit $S$. aureus growth. When extended to fluoroquinolones, this compound also proved efficient in potentiating the effect of norfloxacin by 4-fold in a wild-type strain, being its action directed towards NorA [81].

Orizabins, oligosaccharides from resin glycosides, were evaluated for their efflux pump inhibitory activity on strains of $S$. aureus. These amphipathic compounds showed synergy with norfloxacin, whilst showing no antibacterial activity by themselves. Orizabin IX (46) completely inhibited the growth of a NorA overexpressing strain at $1 \mu \mathrm{g} / \mathrm{ml}$, while orizabin XIX (47) reversed norfloxacin resistance by 4 -fold at $25 \mu \mathrm{g} / \mathrm{ml}$ for the same strain [82]. Murucoidins, which are also resin glycosides, strongly potentiated the action of norfloxacin against a $S$. aureus NorA overexpressing strain by 4 -fold at concentrations ranging from 5 to $25 \mu \mathrm{g} / \mathrm{ml}$. Stoloniferin I potentiated the same activity by 8 -fold at a concentration of $5 \mu \mathrm{g} / \mathrm{ml}$ [83].

A study aiming to find new EPIs from the plant Geranium caespitosum led to the isolation of novel acylated neohesperidosides. It was found that two compounds, compound 48 and 49, were successful at restoring susceptibility to berberine, norfloxacin, and ciprofloxacin at sub inhibitory concentrations, possibly due to NorA inhibition [84].

Capsaicin (50), the major constituent of the fruits of the Capsicum genus, is a known P-gp inhibitor. Studies were performed, in order to evaluate if its inhibitory activity was also applied to MDR pumps in bacteria. In a strain of $S$. aureus overexpressing the NorA efflux pump, and therefore resistant to fluoroquinolones, $\mathbf{5 0}$ proved efficient in increasing the susceptibility to ciprofloxacin, while reducing the emergence of ciprofloxacin-resistant mutants. Molecular modelling studies of the complex capsaicin (50)-NorA, using the glycerol-3-phosphate transporter to predict the structure of NorA, showed that compound $\mathbf{5 0}$ aliphatic chain extended into NorA's hydrophobic cleft. Furthermore, a weak hydrogen bond between Arg-98 and the aryl hydroxyl contributed to the stability of the complex [85].

Studies in the aqueous extract of Artemisia absinthium showed promise in the inhibition of MDR efflux pumps. It was proven that this activity came from caffeoylquinic acids. At a concentration of $20 \mu \mathrm{M}, 4$ '-5'-O-dicaffeoylquinic acid (51) potentiated the activity of berberine by 16-fold and of norfloxacin, by 10-fold against a wild-type strain of $S$. aureus, while not displaying effects in a nor $A$-deleted strain, confirming this compound's NorA inhibition ability [86].

Olympicin A (52) is an acylphloroglucinol, found in the aerial parts of Hypericum olympicum. This molecule had some efflux impeding ability, proven by the improvement of intracellular accumulation of enoxacin in a NorA overexpressing strain of $S$. aureus, at $50 \mu \mathrm{g} / \mathrm{ml}$ [87]. 
Riparins are natural alkylamides found in the plant Aniba riparia. Since the extraction of these compounds comes with very low yields, synthetic approaches were developed. As such, the fundamental core of riparins, Rip-A (53), was synthesized and derivatives were prepared, Rip-B (54) to E (55). Rip-E (55) showed good growth inhibitory activity against $S$. aureus, attributed to their lipophilic nature and the presence of hydroxyl groups at the benzamide moiety. Oppositely, Rip-B (54), with two methoxy groups at the phenyl-ethyl moiety did not present antibacterial activity, presenting instead a decrease in the MIC of ciprofloxacin and norfloxacin, similarly to reserpine. Combined with the ability of decreasing ethidium bromide's MIC, the activity of compound $\mathbf{5 4}$ was attributed to NorA inhibition, suggesting its potential as an EPI [88].

Extracts of Mirabilis jalapa have shown good results in reversing fluoroquinolone resistance in strains of $S$. aureus overexpressing the NorA efflux pump. One compound in particular, $\quad \mathrm{N}$-trans-feruloyl-4'-O-methyldopamine (56), caused an 8-fold reduction in the MIC of norfloxacin, making it a potential lead in the search for structurally similar synthetic alkylamines [89].

Synthesis of derivatives was undertaken, taking $N$-transferuloyl-4'-O-methyldopamine (56) as a model. The aim was to identify active compounds from natural sources and, as such, couplings between cinnamic acid derivatives and natural-occurring amines were chosen. SAR criteria was taken into account, as it is known that substitution of the aromatic rings, methoxy or hydroxyl substitution, double bonds and aromatic ring nature in the amine part influence the activity. The lead compound was $\mathrm{N}$-trans-3,4-Odimethylcaffeoyl dopamine (57) since it showed the same activity as the natural compound. It was found that, for the cinnamic moiety, a hydroxyl substitution on the aromatic ring appears to be better than a methoxy group or unsubstituted derivatives and the double bond is essential for activity. As for the amine part, trisubstitution on the aromatic ring increases antibacterial activity, at the cost of decreased efflux pump inhibition. Methoxy substitution yields compounds with better results than hydroxyl substitution, which was better than no substitution and tryptamine combinations showed the best results (Figure 7). Among the compounds tested, compound $\mathbf{5 8}$ showed potentiation of norfloxacin comparable to that of reserpine (30). At a concentration of $30 \mu \mathrm{M}, \mathbf{5 8}$ showed good results for the inhibition of ethidium bromide efflux. For these compounds, it was shown that the activity reaches its highpoint when the phenyl ring is substituted with two hydroxyls [89].

Grapefruit oil fractionation led to the isolation of three compounds, two coumarin derivatives and one bergamottin derivative. The bergamottin epoxide (59) showed a reduction of ethidium bromide's MIC by 6-fold on different MRSA strains, and a 20-fold reduction in norfloxacin's MIC. This reduction was also achieved when the coumarin epoxide (60) was present, and suggests an activity towards NorA efflux pumps [90].

Coumarins isolated from Mesua ferrea were also studied for their efflux pump inhibitor activity against clinical strains and NorA overexpressing strains of $S$. aureus. Seven coumarins were tested, and showed good results in inhibiting ethidium bromide efflux. Two compounds, 61 and $\mathbf{6 2}$, showed potential EPI activity against the NorA overexpressing strain and a MRSA strain, using norfloxacin as a substrate, which makes them desirable compounds for the synthesis of derivatives [91].

Screening of natural products with P-gp inhibitory effects $(92,93)$ led to the identification of two compounds with NorA inhibitory activity, osthol (63) and curcumin (64) [36, 92-93]. Curcumin (64), derived from the rhizomes of Curcuma longa, has additionally already shown activity as an anti-inflammatory, antioxidant, antiviral, and anticancer agent [36, 92-93]. These compounds were effective in reducing the MIC of ciprofloxacin by 4 - and 8-fold, respectively [94].

A penta-substituted pyridine, 2,6-dimethyl-4-phenylpyridine-3,5-dicarboxilic acid diethyl ester (65), was isolated from the rhizomes of Jatropha elliptica. This compound, when in association with ciprofloxacin, showed a strong effect against NorA efflux pumps in S. aureus [95].

A methanol extract of the plant Dalea spinosa yielded the isolation of six compounds, whose EPI potential was analyzed against wild-type $S$. aureus and mutant strains. It was shown that spinosan A (66), at a concentration of 48 $\mu \mathrm{g} / \mathrm{ml}$, and its acetate 67 , at a concentration $42 \mu \mathrm{g} / \mathrm{ml}$, were able to decrease berberine's MIC by 8 - and 62-fold, respectively, against the wild-type. Other compounds were also able to reduce berberine's MIC, but to a lesser extent. Compound 67 also managed to decrease berberine's MIC in the NorA overexpressing strain, suggesting inhibitory activity in this efflux pump [96].

\subsubsection{Synthetic Compounds}

The INF series (compounds 68-72) resulted from an initial screening of 9600 structurally diverse compounds, in order to find NorA inhibitors for B. subtilis, not expressing Bmr. The NorA substrate chosen was ethidium bromide [67].

Since this screening did not focus on a specific chemical group, the most active compounds were divided into several groups. Several indole derivatives were active, which did not come as a surprise, since reserpine (30) itself has an indole moiety. Another large group was the trichloromethylaminal containing compounds, which were not further explored as their likelihood to be toxic was high. Biphenyl urea derivatives were also active, and other compounds, with no obvious similarities, showed activity as well. Five compounds were selected for further tests, INF 55 (68), INF 240 (69), INF 271 (70), INF 277 (71) and INF 392 (72). These compounds were active at concentrations of $5 \mu \mathrm{g} / \mathrm{ml}$ or less, and included the most potent compound, 72, the most potent indole, 68, and the most potent biphenyl urea, 71. Derivative $\mathbf{7 2}$ was able to reduce resistance to ethidium bromide and ciprofloxacin by 8 -fold at a concentration of 0.4 $\mu \mathrm{g} / \mathrm{ml}$. All five inhibitors were more potent than reserpine (30) at promoting the activity of ciprofloxacin in $S$. aureus and, by being structurally different, it was hypothesized that 
a potent, nontoxic lead would be identified. These compounds also proved effective in inhibiting the Bmr multidrug transporter in B. subtilis and two compounds, 68 and 71, also enhance fluoroquinolone activity in Streptococcus pneumoniae [67].

The results obtained with INF 55 (68) in $S$. aureus as an inhibitor of the NorA efflux pump led to an increased interest in this kind of scaffold. SAR studies regarding the C5 proved that substitution in this carbon is crucial for activity. Substitution with a nitrile group leads to retention of potency, and carbonyl based electron-withdrawing groups at $\mathrm{C}-5$ would result in molecules with no activity [97]. Three dimensional-quantitative structure-activity relationship (3DQSAR) studies predicted that 2-aryl indole derivatives would be NorA inhibitors. Functionalized INF 55 (68) showed a decreased MIC for berberine, a MDR transporter substrate. Berberine is an amphipathic cation alkaloid. From the series of 2-aryl-5-nitroindoles synthesized by Samosorn et al. (2009), the alcohol $\mathbf{7 3}$ was the most effective in inhibiting berberine efflux, as well as other antibiotics, such as ciprofloxacin, in S. aureus [98]. Later, the same group developed a strategy that consisted in a molecule presenting dual action: an antibiotic and a MDR inhibitor. Therefore, they combined berberine, a hydrophobic cation that is a MDR substrate, with INF 55 (68), a MFS inhibitor. These efforts yielded compound 74 [17-18, 99]. In terms of antibacterial activity in $S$. aureus, the hybrid was about 100 times more active than berberine alone, and the difference was even higher when tested against a NorA overexpressing strain of $S$. aureus. Enterococcus faecalis also presented resistance to berberine, but was susceptible to 74. [99] Synthesis of sulfur derivatives was achieved based on the premise that the activity of $\mathbf{6 8}$ is less dependent on the nitro group than the 2-arylindole. Benzothiophene, thiophene, and benzofuran derivatives, such as compounds 75, 76, and 77, respectively, were able to synergize with ciprofloxacin and ethidium bromide, increasing their intracellular concentration and leading to the conclusion that these compounds inhibit NorA in S. aureus. [100]

Taking into account the fact that the indole $\mathrm{NH}$ is not essential for efflux pump inhibition, Samosorn et al. (2009) simplified the indole moiety of berberine-based hybrids, along with the effect of a methylene ether linking group. Their results showed that a hybrid molecule with oxygen in the linkage chain had stronger antibacterial and MDR pump inhibitory activity than the original 74 [101]. On the other hand, removal of the indole moiety produced compounds with lower dual activity, and this structural simplification led to compounds with low antibacterial activity, but with retained NorA pump inhibitory activity. These data suggest that while the indole moiety is not necessary for NorA pump inhibition, it is required for the antibacterial activity of hybrid compounds [101].

A recent study identified a novel class of NorA EPIs with a polysubstituted indole moiety. Twenty molecules were highly active in inhibiting ethidium bromide efflux in $S$. aureus. However, the best results were obtained with compounds $\mathbf{7 8}$ and 79, which were derivatives that presented three and four carbon atom chains, respectively. Compound 78 presented the best half maximal inhibitory concentration $\left(\mathrm{IC}_{50}\right)$, with a concentration of $1.8 \mu \mathrm{M}$. Compound 79 exhibited similar $\mathrm{IC}_{50}(2 \mu \mathrm{M})$ and ethidium bromide inhibition as 78. Compounds with shorter or longer carbon chains did not present such good results, leading to the conclusion that three or four carbons make up the right distance between the indole scaffold and the basic center, thus presenting the best inhibitory effect. When tested for their synergy with ciprofloxacin, both $\mathbf{7 8}$ and $\mathbf{7 9}$ were able to restore the antibiotic's activity [102]. It was found that the substitution of the $\mathrm{C}-5$ in the indole results in a potent EPI, particularly if there is a propoxyl chain carrying terminal cyclic amino groups, which appears to be crucial for NorA inhibition at low concentrations. The presence of the $\mathrm{N}$ benzyl moiety preserves inhibition and contributes to the modulation of the biological effects and ADME properties, depending on its substituents [103].

Another successful example of a hybrid antibacterial/EPI is the fluoroquinolone scaffold accommodating a bis-aryl urea EPI moiety at the C-7 position. This kind of compounds was successful in inhibiting NorA pumps in $S$. aureus. Compound 80, which incorporates a bis-aryl urea motif into the ofloxacin core, proved to be a potent inhibitor of MFS efflux pump systems, achieving $84 \%$ inhibition rate at 10 $\mu \mathrm{M}$. Moreover, these compounds also successfully inhibit MepA pump systems from the MATE family [17-18, 104]. Fluoroquinolones containing a thiopyranopyridine moiety at the C-7 position were synthesized and their activity was tested against strains of $S$. aureus. However, these derivatives did not seem to be NorA substrates. Further investigations into thiopyranopyridinylquinolone esters were carried out. Some of the synthesized compounds displayed better inhibitory activity than reserpine (30), namely quinolone esters 81, 82 and $\mathbf{8 3}$, and showed significant MIC reduction of ciprofloxacin. Compounds $\mathbf{8 2}$ and $\mathbf{8 3}$ also showed a 32-fold MIC reduction of ciprofloxacin for strains of $S$. aureus overexpressing MepA pumps from the MATE family $[18,105]$. In terms of established SAR, these compounds definitely do not need the fluorine atom at the $\mathrm{C}$ 6 position for the antibacterial activity of thiopyridinylquinolones. A methyl at the C-8 position yields a compound with enhanced activity against Gram-positive bacteria for 6-amino derivatives. The thiopyranopyridine moiety at the C-7 position is crucial for NorA and MepA inhibition (Figure 8) [105].

Derivatives of 2-(4-propoxyphenyl)quinolone were synthesized based on the premise that its large hydrophobic area and ability to establish an electrostatic interaction would make them suitable inhibitors of NorA. These derivatives also mimic the quinolone antibacterial core and possess a versatile scaffold, making it prone to be very simply chemically modified. These classes of compounds were obtained by performing modifications in the 2-phenyl-4Hchromen-4-one moiety, which is a common feature of flavone and flavolignane EPIs [106].

Studies showed that the $O$-substituted 2-phenyl-4hydroxyquinoline derivatives displayed the best activity as 
NorA inhibitors. Compounds 84 and 85 showed synergistic activity against a strain of $S$. aureus overexpressing NorA efflux pumps comparable to reserpine (30) and, comparing the results obtained with a mutant strain, absent of NorA, it was found that these compounds are able to completely restore the antibacterial activity of ciprofloxacin in the resistant strain $[18,106]$. Preliminary SAR studies for this new class of EPIs showed that the best activity is when the quinolone core is replaced with the 2-phenyl-4hydroxyquinoline group with an alkylation in the C-4 hydroxyl group. 2-Ethylamino chains inserted at the $\mathrm{N}-1$ position of the quinolone nucleus or at the C-4 hydroxyl of the quinoline moiety provided compounds with better activity than those carrying the same chain at the $\mathrm{C}-2$ of the phenyl ring. The best substituent for the C-2 phenyl ring appears to be the C-4' propoxy group (Figure 9) [106]. Taking these results into account, efforts have been placed to obtain more potent NorA inhibitors, introducing $O$-alkyl or different $O$-alkylamino chains at the $\mathrm{C}-4$ position. The strategy was to determine the essential 3D structural requirements for the inhibition of NorA, based on the NorA EPIs described in literature through the use of computational methods, since the 3D structure of this pump was not elucidated yet. The results led to the building of a possible pharmacophore, which was used to design novel compounds, with different substituents at the C-4 position, which were synthesized and biologically evaluated [107]. The compounds were assayed for their ability to reduce the MIC of ciprofloxacin in $S$. aureus strains, and all compounds displayed this decrease for the strain overexpressing NorA pumps. One compound in particular, 86, showed a 16-fold reduction in this strain for ciprofloxacin's MIC. SAR studies for this emerging class show that the best activities come from the compounds bearing the 2-ethylaminoalkyl chains linked to the C-4 hydroxyl group, such as compound 87. The inclusion of an aliphatic ring including the side chain nitrogen atom led to retention of activity, whereas inclusion in an aromatic ring led to loss of activity, where the nitrogen nucleophilicity was reduced. The mono-substituted compounds, like $\mathbf{8 8}$, with a benzyl group were well tolerated, and showed higher ethidium bromide inhibitory activity than compounds with two benzyl groups as substituents (Figure 9) [107].

3-(Dihydronaphtyl)-propenoic acid amines were evaluated for their EPI activity. Five compounds were synthesized, and two of them showed good results in inhibiting ciprofloxacin efflux in strains of wild-type and NorA overexpressing $S$. aureus. Further studies were performed, given the low EPI activity of this kind of compounds. Fifteen different compounds were synthesized, taking $\alpha$-tetralone (12) as starting material. Out of these, four compounds showed significant MIC reductions for ciprofloxacin for the NorA overexpressing strain. One compound, 89, even displayed a 16-fold reduction, while other remained in the range of 4- to 8-fold. These studies allowed SAR to be established. Addition of methoxy or allyloxy substituents at the 6,7position of the 3,4-dihydronaphtalene leads to lower or no activity, whereas no substitution originated better EPIs.
Saturation of the double bonds lowered the potentiating activity (about 2-fold), while unsaturated derivatives made much more potent compounds [108].

Boronic species have been described for a variety of activities, namely antimicrobial and antineoplasic. Organoboron compounds, oxazaborolidines, boronic esters, and boronic acids are of particular interest, within this class. Fontaine et al. (2014) developed studies on these molecules, starting from a screening of a library of approximately 150 compounds, out of which 24 were hit compounds (exemplified with compounds 90 and 91), and were able to restore the activity of ciprofloxacin by 4-fold at concentrations of 0.5 to $8 \mu \mathrm{g} / \mathrm{ml}$ against the NorA overexpressing strain of $S$. aureus [109]. Preliminary SAR studies demonstrated the need of the boron atom for activity. Most pyridine-3-boronic acids showed best potentiating activity (e.g., compound 90), whereas pyridine-4-boronic derivatives showed no activity, with exceptions, including a fluorinated ester. The shift of the boronic moiety from C-3 to $\mathrm{C}-4$ led to a loss of activity and the trigonal boronic acid function gave better results that the sodium boronate salts and the potassium trifluoroborate. Concerning the pyridine3 -boronic acids, the most promising compounds appear to be the 6- and the 5,6-disubstituted ones, being the latter compounds less potent. Cyclisation at the C-5 and C-6 positions was well tolerated, as was the introduction of a methyl at the C-5 position. For the benzene analogues, which displayed good activity, cyclisation at the C-3 or C-4 was also well tolerated, as was the introduction of a methyl. A substituent at the para position to the boronic moiety proved to be very important. Two compounds were particularly efficient in inhibiting NorA, and did not display intrinsic antibacterial activity and cytotoxicity: the 6benzyloxypridine-3-boronic acid 90, which displayed the highest activity, potentiating ciprofloxacin by 4-fold at a concentration of $16 \mu \mathrm{g} / \mathrm{ml}$, and the 4-benzylxybenzene boronic acid 91 [109].

Compound 90, being the most promising compound, was considered a hit, and its structure was used as a model for the synthesis of new derivatives. Therefore, the 6-benzyloxy group was substituted with (aryl)alkoxy chains with variable lengths (compounds $\mathbf{9 2}$ and $\mathbf{9 3}$ as examples), in an attempt to explore the putative hydrophobic binding site of NorA, and substituents were added to the phenyl ring. The ether linkage between the pyridine ring and the 6-benzyloxy group was substituted as well. The first substitution made was the replacement of the 6-benzyloxy group with alkoxy chains of diverse lengths, as to explore the size of the hydrophobic pocket of NorA. The potency of the compounds increased with the increase of the size of the side chain, although the compound with a 11-carbon side chain displayed no activity whatsoever. A new series of compounds with a phenyl ring at the end of the side chain were synthesized, with the aim of enhancing hydrophobicity, and the results showed these compounds were more efficient. It was concluded that the compound with the best performance possessed a side chain with seven carbon atoms and a phenyl group: compounds $\mathbf{9 2}$, 6-(3-phenylpropoxy)pyridine-3-boronic acid, and 93, 6-(4- 
phenylbutoxy)pyridine-3-boronic acid, showed the highest activity in the potentiation of ciprofloxacin (4-fold). The introduction of substituents in the phenyl ring of compound 90 led to unsatisfactory results, with the best compound displaying moderate antibacterial activity. A methyl group in the $\mathrm{C}-5$ position did not show influence on the activity, and the modification of the ether linkage led to varied effects: introduction of a sulfanyl, thus conserving the H-bonding acceptor capacity, led to similar potentiating activity, whereas the introduction of an amine linkage, an H-bond donor, led to a decrease in the activity. Finally, the shift of the benzyloxy group from the $\mathrm{C}-6$ to the $\mathrm{C}-5$ resulted in no activity [110].

Compounds AE-848/42434549 (94) and AN-465/42885978 (95) were hits in a virtual screening of novel NorA efflux pump inhibitors in $S$. aureus. The structures deemed as active in silico were later synthesized and evaluated on their ability to prevent ethidium bromide efflux, as well as ciprofloxacin potentiation. Compound 94 and 95 were as potent as reserpine (30) for ethidium bromide efflux and more potent at synergizing with ciprofloxacin. These hit compounds 94 and 95 paved way for the synthesis of new analogues, which allowed SAR studies to be made, in order to assess which parts of their structure were determinant for the inhibition of NorA. For compound 94, it was shown that limited modifications to the sulfone and amide group do not cause significant changes in activity. On the other hand, modifications to the imidazole ring result in a reduction of activity. Replacement of the 1-methylpyrrole ring with a furan also shows a decrease in the activity of the compound. As for compound $\mathbf{9 5}$, the $O$-benzyl group is essential and the introduction of a benzyl in the secondary nitrogen leads to a complete loss of activity. Furthermore, the methyl group of the 2-(1-phenyl-1-propanol) moiety can be replaced with a 2(1-phenyl-1-ethanol) portion without a decrease in the activity [111].

\subsubsection{Existing Drugs}

Several drugs already available for the treatment of various diseases have shown potential to be used as MFS EPIs. Reserpine (30), mentioned before, is one of them. Other drugs include verapamil, omeprazole, paroxetine and chlorpromazine. Even though bacterial MFS transporters and mammalian ABC efflux systems, as P-gp, lack structural homology, they share similar substrate profiles, which means that mammalian MDR inhibitors can also present bacterial efflux inhibition. However, the concentrations needed for efflux pump inhibition are too high, which means that toxic effects can arise if these compounds are used for this purpose [18], [112]. In fact, the addition of verapamil to chemotherapy was accompanied with cardiac arrhythmia and hypotension [113]. This leads to the requirement of developing analogues of these existing drugs maintaining the desired EPI activity at concentrations that do not display toxicity [112].

The antiarrhythmic verapamil, a known P-gp inhibitor, has shown moderate NorA inhibitory activity in both $B$. subtilis and $S$. aureus, enhancing fluoroquinolone activity [114-115]. However, the best results were obtained with proton pump inhibitors omeprazole (96) and lansoprazole. Therefore, a series of pyrrolo[1,2-a]quinoxaline derivatives mimicking the omeprazole structure (96) were designed, with the aim of evaluating their EPI activity against NorA. All the eleven derivatives showed a reduction in norfloxacin's MIC, being compound 97 the most active, reducing the MIC by 16-fold at $128 \mu \mathrm{g} / \mathrm{ml}$. These compounds did not show disturbance of the electrical potential and the transmembrane $\mathrm{pH}$, excluding the alteration of the proton-dependent pump as the mechanism of action. Instead, it is thought that these compounds interact directly with NorA. It was shown that the benzimidazole moiety is critical for the activity of these compounds (Figure 10), and replacing it with an imidazole, a pyridine or a pyrrolo[1,2-a]quinoxaline ring resulted in loss of potency. Furthermore, if there is a methoxysubstituent in the benzimidazole ring, potency increases. Introduction of chlorine in the C-7 of the pyrroloquinoxaline heterocycle provided an efficient restoration of norfloxacin's bactericidal activity. Finally, the benzimidazolyl group was also important for activity [112].

This was not the first time that pyrrolo[1,2-a]quinoxaline derivatives were described as EPIs. In fact, a previous study by the same group produced 12 new 4-[116]pyrrolo[1,2$a$ ]quinoxalines and one pyrrolo[1,2- $\alpha]$ thieno[3,2-e]pyrazine compound. Two compounds, 98 and 99, were more active than reserpine (30), diminishing norfloxacin's MIC by 16fold in a NorA overexpressing strain of $S$. aureus. This study showed that 4-[116]pyrrolo[1,2-a]quinoxalines and their 7methoxy analogues were more active, in contrast with the chlorine- and 8-phenyl-substituted compounds, that presented the lowest effects. Replacement of the $N, N$ diethylamino group by pyrrolidine, its isomer with restricted conformation, led to enhanced EPI activity, whereas replacing it with a piperidine ring led to more flexible and, consequently, less active compounds. The pyrrolothienopyrazine 99 moiety yielded the best results, suggesting that sulfur, an electron-rich atom, potentiates the EPI activity [116].

GG918 (19) was discovered in a screening program aimed to identify inhibitors of mammalian P-gp, and was able to increase the concentrations of paclitaxel. This compound did not present antibacterial activity against the strains of $S$. aureus tested. However, the co-administration with norfloxacin resulted in a 4-fold reduction in the MIC of the NorA overexpressing strain [52].

Two mammalian MDR inhibitors, biricodar (100) and timcodar (101), confer increased drug sensitivity to cells expressing both the P-gp and multidrug resistance protein (MRP)-1 efflux systems. They also show potentiation of multiple antibiotics in Gram-positive bacteria. In fact, both compounds were able to potentiate the activity of ethidium bromide against $S$. aureus, E. faecalis, and $S$. pneumoniae. For the specific case of $S$. aureus, both compounds were able to partially reverse fluoroquinolone resistance. The fact that the MIC of ethidium bromide was non-saturable for the three 
bacteria tested suggests that $\mathbf{1 0 0}$ targets multiple efflux pumps [117].

A series of polysubstituted pyrroles was screened for their Pgp inhibition activity, which made way for the identification of a lead, and the compounds were further investigated for their bacterial efflux pump inhibition activity. A simplified pyrrole alkaloid, 102, displayed dual activity as both a P-gp and a NorA inhibitor against $S$. aureus. This compound led to 4-fold improvement in the MIC of ethidium bromide in the NorA overexpressing strain. Compound 102 was also able to reduce ciprofloxacin's MIC by 8 -fold when at $50 \mu \mathrm{M}$ [118].

Tariquidar (103), a third generation MDR modulator, with activity in inhibiting P-gp and BCRP efflux systems, has shown promise in inhibiting efflux pumps in $S$. aureus. In fact, this compound showed an increase of the isotopic form of ciprofloxacin in all investigated strains, displaying best results in the strain that overexpresses the NorA pump. [119] The class of phenylpiperidine selective serotonin reuptake inhibitors (PSSRI), which includes the antidepressant paroxetine (104), showed efflux-related potential against $S$. aureus, particularly that conferred by NorA. In fact, paroxetine and its isomer, 105, showed potency with respect to inhibition of this efflux pump against ethidium bromide. The isomer $\mathbf{1 0 5}$ also interfered with the accumulation of norfloxacin by NorA, reducing by 4 - to 8 -fold its MIC. [120] Femoxetine (106) is a paroxetine derivative, with activity as an EPI. [120] Studies were performed as to determine which moieties in paroxetine- and femoxetine-like PSSRI were detrimental for efflux pump inhibition in S. aureus, and conclusions from SAR are highlighted in Figure 11. Synthesis of derivatives showed that the fluorine atom on the phenylpiperidine moiety is not associated with the activity. The $N$-substitution of the piperidine ring yields different kinds of activity, suggesting that the NH group forms a positive binding contact with the target pump, which does not happen if there is a $N$-substitution (compound 106). The phenyl ether substituents also play an important role as determining the EPI activity. [121]

Also for this kind of compounds, a study was performed, in order to conclude whether the 4-phenyl ring is required for EPI activity and the effects of changes in the two-atom aryloxymethyl linker, replacing it with a thioether, amine, and alkene linker portions. Compounds 107 and $\mathbf{1 0 8}$ displayed the lowest MIC against $S$. aureus. Synthesis of multiple derivatives proved that the 4-phenyl moiety is not vital for EPI activity and that a variety of two-atom linker groups for the 3-aryl piperidine moiety can maintain EPI activity [122].

The ciclooxigenase-2 (COX-2) inhibitor celecoxib (109) has also received attention for its MDR inhibitory activity. There had been a link between COX-2 and $m d r 1$ gene expression, which has consequences in cancer chemo resistance, and the use of celecoxib (109) reversed drug resistance. If this is true for mammalian efflux pumps, for bacteria there is no evidence of a COX-2-like gene, suggesting that celecoxib acts differently in inhibiting MDR in bacteria. A study was carried out in order to prove this hypothesis, which demonstrated that this drug helped in increasing sensitivity to antibiotics in S. aureus and Mycobacterium smegmatis. These effects probably occur due to the blockage of MDR transporters involved in the efflux of antibiotics, such as ampicillin, ciprofloxacin, kanamycin, and chloramphenicol. The observation of the accumulation of ethidium bromide intracellularly leads to the conclusion that this compound acts as a NorA inhibitor [123].

As celecoxib (109) demonstrated such promising activity, analogues were tested, which bore low or none antiinflammatory activity and possessed the 1,4dihydropyrazolo[4,3-c]-benzothiazine-5,5-dioxide nucleus. Ethidium bromide efflux inhibition was tested first in silico, and the 17 molecules that fit the chemical space requirements were then reduced to six, based on the activity prediction. These compounds were then screened for their in vitro NorA inhibitory activity, using an overexpressing strain of $S$. aureus. One derivative in particular, compound 110, showed the best activity concerning ethidium bromide efflux $(76.9 \%)$ which is better than celecoxib (109) $(64.5 \%)$. When in combination with ciprofloxacin, $\mathbf{1 1 0}$ displayed results comparable to reserpine (30) and better than paroxetine (104), which makes it a lead compound for the development of inhibitors of this kind [124].

Phenothiazine and thioxanthene derivatives are currently used as neuroleptic and antiemetic agents, and have been described as modest, but broad, antibacterial agents. Although their antibacterial activity is not enough to be clinically relevant, they have shown synergy with standard antibiotic agents, and have also been associated with the inhibition of eukaryotic MDR efflux pumps, such as P-gp. The mechanism through which these compounds exert their antimicrobial potentiation is not yet fully understood. However, it is thought that it might have to do with the inhibition of efflux pumps [125]. A study focusing on the activity of several compounds of these families against $S$. aureus was carried out, with the main aim of showing their potential in inhibiting NorA. The compounds tested, chlorpromazine, fluphenazine, thioridazine, prochlorperazine (111), cis(Z)-flupentixol, and $\operatorname{trans}(E)$-flupentixol (112), displayed intrinsic antimicrobial activity and good results when ethidium bromide efflux was assayed against a NorA overexpressing strains of $S$. aureus. Additionally, phenothiazine 111 and thioxanthene $\mathbf{1 1 2}$ were also able to reduce the proton motor force of $S$. aureus, by reducing the transmembrane potential [125].

Since the phenothiazine moiety proved itself to work as a template for the synthesis of new MDR EPIs, it was chosen to be the scaffold for derivatives with improved activity. The logic for these studies lied in the elimination of the structural features that were responsible for neuroleptic activity. As such, drastic modifications were made, such as the elimination of one ring of the tricyclic benzothiazine backbone and of the chain linked to the N-10 atom, a tertiary amine whose protonation is crucial for the interaction with the dopaminergic receptor, and the addition of a substituted phenyl ring at the $\mathrm{C}-3$ position, to guarantee better lipophilicity. Therefore, a prototype of new 3-phenyl-2H- 
1,4-benzothiazines was developed. Preliminary screening on the derivatives using a wild-type $S$. aureus strain, both with and without ciprofloxacin, showed variable intrinsic and synergistic activity. Two compounds, 113 and 114, displayed the best activity, while displaying no and weak antibacterial activity, respectively. These compounds were able to inhibit NorA efflux of ciprofloxacin, being suggested that 114 was even able to completely inhibit this pump [126].

Thioridazine (115), a phenothiazine, was also tested for its antibiotic potentiation, either in racemate or in its enantiomeric forms. All the forms were able to reduce the MIC of oxacillin in $S$. aureus and erythromycin in Streptococcus pyogenes, being the L-enantiomer more effective in the last case [127-128].

\section{RND}

RND pumps are found mostly in Gram-negative bacteria, being the most responsible pumps for MDR in this kind of organisms. They present a wide variety of lipophilic and amphiphilic substrates, which include several classes of antibiotics, antiseptics, dyes, and detergents. RND transporters present a unique tripartite complex, constituted by a minimum of twelve transmembrane segments: the transmembrane pump, the outer membrane channel, and the periplasmic adaptor protein, which puts the other two components in contact. This structural organization makes the extrusion of substrates directly into the external medium possible [32, 38]. Even though it has always been thought that RND transporters were exclusive to Gram-negative bacteria, it has recently been identified and characterized FarE in $S$. aureus, homolog to the AcrB protein in E. coli [129-130].

Within this family, the most studied system is the AcrABTolC, from E. coli. This efflux pump is composed by three essential parts: a resistance-nodulation-division transporter, AcrB; a membrane fusion protein, AcrA; and a multifunctional outer membrane channel, TolC [7, 131-133]. It transports a wide variety of toxic compounds from the intracellular space directly into the medium, evading the periplasm, and using a proton gradient as the energy source [132-133]. Pseudomonas aeruginosa also possesses an efflux system of the RND family, the MexAB-OprM efflux pump, which confers resistance to a wide variety of antibiotics. This was the first and best characterized efflux system in bacteria. It was found that AcrB (Figure 12, PDB 4ZLJ) is homolog to MexB (Figure 12, PDB 2V50), being their structure very similar [7, 134].

Figure 13 comprises all the RND pump inhibitors that will be discussed in the following section.

\subsection{RND inhibitors}

\subsubsection{Natural Products and Derivatives}

One study revealed that the essential oil of Helichrysum italicum can reduce the resistance to chloramphenicol in $E$. aerogenes, $P$. aeruginosa, and A. baumanii. These data suggested that one or more EPIs are present within the essential oil since it was shown that geraniol (116) produced significant restoration of sensitivity to chloramphenicol by 16-fold, and rendered a total sensitivity of the organism when combined with of phenyl-arginine $\beta$-naphtylamide $(\mathrm{PA} \beta \mathrm{N})$, which will be discussed further ahead [135].

A study published by Aparna et al. (2014) had the aim of identifying natural compounds from plants that present efficacy in potentiating effects of antibiotics in $P$. aeruginosa overexpressing MexAB-OprM and E. coli overexpressing AcrAB-TolC through in silico virtual screening and pharmacophore approaches [136]. The compounds with the best in silico results were then tested for their EPI activity. The two compounds that showed the best activity in potentiating the activity of carbenicillin and levofloxacin in $P$. aeruginosa and $E$. coli were lanatoside $\mathrm{C}$ (117) and daidzein (118). Lanatoside $C$ (117) is cardiac glycoside that inhibits the $\mathrm{Na}^{+}-\mathrm{K}^{+}$-ATPase, which can also be the explanation for its EPI activity against MexB and AcrB. Daidzein (118) is an isoflavone that has previously showed slight EPI activity in Mycobacterium smegmatis and as a modulator of P-glycoprotein, in human cervical carcinoma KB-V1 cells [136].

An in silico study by Ohene-Agyei et al. (2014) identified five phytochemicals that could be docked similarly to $\mathrm{PA} \beta \mathrm{N}$ in the binding pocket of AcrB. Of these five, three of them were able to decrease resistance to antibiotics by inhibiting the AcrAB-TolC system: plumbagin (119) increased sensitivity to erythromycin, chloramphenicol, and tetraphenylphosphonium; nordihydroguaretic acid (NDGA) (120) potentiated the activity of erythromycin, chloramphenicol, tetraphenylphosphonium, novobiocin, and tetracycline; and shikonin (121) decreased resistance to tetraphenylphosphonium [137].

A study proved the usefulness of curcumin (64) as a RND EPI at a concentration of $50 \mu \mathrm{g} / \mathrm{ml}$, increasing the sensitivity of carbenicillin, ceftazidime, and meropenem in $P$. aeruginosa, in decreasing order. It also managed to circumvent the resistance to gentamicin and ciprofloxacin in resistant isolates, which $\mathrm{PA} \beta \mathrm{N}$ failed, leading to the assumption that curcumin inhibits efflux pumps differently from PA $\beta N$ [138].

A screening of 85000 microbial fermentation extracts derived from 3600 strains of actinomycetes and 3500 strains of fungi was made, with the aim of discovering EPIs that increased sensitivity of levofloxacin in $P$. aeruginosa overexpressing the MexAB-OprM or the MexEF-OprN pump. Two compounds, EA-371 $\alpha$ (122) and EA-371- $\delta$ (123), produced by a strain of Streptomyces sp., demonstrated inhibitory activity against MexAB-OprM [139].

\subsubsection{Synthetic Compounds}

The first compound with potent inhibition of RND efflux pumps was PA $\beta N$ (124), a dipeptide amine. This dipeptide was identified as a hit compound from the screening of 200 000 samples of small molecules that potentiate the activity of 
antibacterial levofloxacin against strains of $P$. aeruginosa that overexpressed MexAB, MexCD and MexEF pumps [28, 140]. Compound 124 is a peptidomimetic, developed for using in adjunctive therapy. This compound is a substrate for RND pumps, acting as a competitive inhibitor of multidrug efflux systems, such as MexB, MexD, and MexF, binding to the substrate pocket, impeding antibiotic binding and/or extrusion. Particularly, this EPI binds in a location close to the antibiotic binding site, generating steric hindrance, making it more difficult for the antibiotic to bind. In other words, less quantity of antibiotic is exported, making its intracellular levels higher, thus restoring antibiotic sensitivity [11, 25, 27-28, 141-143]. However, 124 can affect the integrity of the membrane when used in high concentrations, making it prone to the emergency of resistant profiles, namely the modification of the lipopolysaccharide structure, leading to changes in drug penetration [24].

$\mathrm{PA} \beta \mathrm{N}$ (124) has been validated against the AcrAB-TolC in Klebsiella pneumonia, E. coli, Salmonella enterica serovar Typhimurium and Enterobacter aerogenes, and in multiple homologous systems including Acinetobacter baumanii, Campylobacter jejuni, and Campylobacter coli $[11,142]$. Its potency has been demonstrated against the norfloxacin resistance conferred by the Mex efflux system of Pseudomonas aeruginosa, the AcrAB efflux systems of the Enterobacteriaceae, and the erythromycin efflux system of $C$. jejuni [19].

Studies of SAR demonstrated that the basic middle amino acid, arginine, was detrimental for activity, although a substitution with L-lysine showed the same potency as the lead, and the introduction of L-ornithine, originating compound L-Phe-L-Orn- $\beta$-Na, showed a 2 -fold increased potency. As the ornithine moiety provided a simpler synthesis, it became the standard middle residue in successive compounds [25, 142, 144]. However, these compounds were not stable upon incubation with human serum, due to the occurrence of cleavage of peptide linkage between both amino acids, which is explained by the fact that natural (L) amino acids are contained within its structure. In order to overcome this problem, methylation of the NH that links both amino acids was achieved, maintaining potency and being this derivative able to resist serum proteases. Serum stability was also increased by replacing the L-amino acid with $\mathrm{D}$-amino acid [25, 142]. In the same study, the authors also proved that the amino acid in the aal position (Figure 13, 124) should be appropriately substituted, and the replacement of phenylalanine with a homophenylalanine led to improved potency. The problem with this compound was the fact that the ornithine moiety could easily form a lactam, rendering the compound inactive. This was overcome by switching the L-ornithine with the Lphenylalanine, which originated L-Orn-L-hPhe- $\beta-\mathrm{Na}$, which originated a compound just as potent, but with no propensity to form a lactam. As for the $\beta$-aminonaphtalene moiety, it was proven that replacing it with 3 -aminoquinoline led to reduced cytotoxicity and intrinsic antibacterial activity, even though its potency was slightly reduced [25, 142]. These efforts originated the compound L-Orn-L-hPhe-3-NHQ
(125). In conclusion, the analogues synthesized by Renau et al. (1999) did not show improvements in potency when compared to PA $\beta N$ (124), but showed increased stability [142].

The same group developed further studies with this type of molecules and demonstrated that conformational restriction of the amino group of ornithine showed less toxicity, while maintaining potency. Such compounds were attained by adding a proline substituent, thus incorporating the two basic residues necessary for activity, which resulted in the successful synthesis of constrained derivatives, more potent and less toxic than $\mathbf{1 2 5}$. The most interesting derivative, compound 126, was as potent as $\mathbf{1 2 5}$, but more than 4 -fold less toxic. This compound also retained the activity against RND efflux pumps MexAB-OprM, MexCD-OprJ and MexEF-OprN. Although this compound showed similar protein binding in rats as $\mathbf{1 2 5}$, its pharmacokinetic parameters were improved, and its efficacy in combination with levofloxacin was demonstrated in an in vivo model [145].

In a latter study developed by the same group, several nonpeptide analogues were synthesized, and it was hypothesized that the peptide backbone is not essential for the activity of this type of compounds. The results suggested that the inhibition of pumps was possible due to the di-cationic nature of the compound, the appropriate lipophilicity and a disposition similar to that of compound 125 [146].

MBX2319 (127) is a pyranopyrimidine EPI inhibitor of AcrAB-TolC efflux pump in E.coli and other Enterobacteriaceae [141]. Compound $\mathbf{1 2 7}$ did not show any antibacterial activity, which is a positive feature for EPIs. It also potentiated the antibacterial activity of fluoroquinolones, such as ciprofloxacin and levofloxacin, and $\beta$-lactams, such as piperacillin against strains of $E$. coli that presented AcrAB-TolC efflux pumps [28, 141]. In $P$. aeruginosa, although compound 127 presents activity against RND-type pumps, it is unable to penetrate the outer membrane, which is highly selective [28].

In $E$. coli, the target of compound $\mathbf{1 2 7}$ is the membrane transporter AcrB. This molecule binds to the hydrophobic trap of the $\mathrm{T}$ protomer, interacting with the hydrophobic residues that constitute the deep binding pocket as a hydrophobic trap. $\pi-\pi$ Interactions also occurs, between the pyridine ring of $\mathbf{1 2 7}$ and the aromatic side chain of Phe-628. The phenyl and morpholinyl groups also interact with Phe178 and Phe-615. The dimethylenesulfide linker and the geminal dimethyl group present van der Waals interactions between the side chain of Phe-178, and Tyr-327 and Met573, respectively [28, 147].

SAR studies demonstrated that the maintenance of activity depended on the geminal dimethyl moiety of the tetrahydropyran, the nitrile group and the length of the dimethylenesulfide linker. This data is presented in Figure 14. On the other hand, the morpholine moiety and aryl group are both prone to substitution, originating more stable derivatives in terms of activity, solubility, and metabolism [28]. Studies with this scaffold have shown that non-acidic substituents can be added to the phenyl group, in order to 
improve potency and CYP450 inhibition, and modifications to the morpholinyl group has greater effects on solubility and stability [140].

Analogues were synthesized by Sjuts et al. (2016), by changing the structure and functional groups of compound 127 (148). They reached the conclusions that the introduction of a 2,6-dimethyl to the morpholinyl group improved both microsomal stability and EPI activity. The replacement of the morpholinyl group with 2methoxyethylpiperazinyl improved aqueous solubility, at the cost of a slight reduction of EPI activity. Two analogues, 128 and 129, resultant of the combination of 2,6dimethylmorpholinyl and acetamide or acrylamide, respectively, on the phenyl group, resulted in a 10-fold to 20fold increase in EPI activity against E. coli, respectively, comparing to MBX2319 (127) [28, 148]. The increased EPI activity of these compounds has its basis in the acetamide and acrylamide groups and the morpholinyl group. The acetamide groups are engaged in highly ordered and complex hydrogen bonds, with its center in a solvent water molecule, which plays the role of a hydrogen bond donor to the carbonyl backbone oxygen of Ala-286 and Gln-151 side chain of AcrB. The acrylamide group forms a bridging hydrogen bond through a water molecule to the side chain of Gln-176 [28].

Nguyen et al. (2015) also synthesized a series of analogues, with the morpholine scaffold substituted by a 2,6dimethylmorpholinyl group (140). Many of these analogues exhibited a solubility improvement of 10 -fold, compared to compound 127. The most potent and promising compounds of these series were compounds 130, 131, 132, 133 and 134 [140].

Through a screening of an $N$-heterocyclic organic compound library, Bohnert and Kern (2005) were able to find a novel class of EPIs, the arylpiperazines [25, 149]. The aim of their work was to find compounds that would potentiate levofloxacin potency against strains of $E$. coli overexpressing $\operatorname{acr} A B$ and acrEF. This was fulfilled when they found phenylpiperazine derivatives with promising activity [25, 149]. SAR studies suggested that elongation of the spacer between the benzene ring and the piperazine ring would enhance potency. Also, substitutions on the benzene ring by halogen atoms led to an increase in potency [24, 149]. On the other hand, the potency would decrease when an ethyl or phenyl group was added to the piperazine ring. With this, the authors found the most potent phenylpiperazine, meta-TFMPP (135), and naphthylpiperazine, 1-(1-naphthylmethyl)-piperazine (NMP) (136) [149].

NMP (136) was the most potent unsubstituted arylpiperazine, able to increase the intracellular concentration of chloramphenicol, tetracycline, linezolid, fluoroquinolones, and macrolides. Although it does not display action in $P$. aeruginosa, it shows effectiveness in $A$. baumanii and several Enterobacteriaceae [11, 23]. EPI activity was also reported in clinical isolates of $E$. coli, most relevantly in fluoroquinolone resistance [150]. Through computational analysis, it was found that NMP (136) binds with high affinity to the lower part of the deep pocket of the $\mathrm{B}$ monomer, overlapping in part the binding site of chloramphenicol [11]. The binding site for NMP (136) includes interactions with hydrophobic residues near the hydrophobic patch and Gly-617 of the G-loop. This data suggests that NMP (136) interferes with the movement of the G-loop, important for extruding substrates, thus inhibiting the action of AcrB [25].

Nakayama et al. (2003) performed a series of syntheses in order to attain a compound specific for the inhibition of a MexAB-OprM specific EPI [151-152]. They identified a hit, compound 137, through high-throughput screening for levofloxacin potentiation using a strain of $P$. aeruginosa strain overexpressing MexAB-OprM. The features this group was looking for was the effective inhibition of efflux pump and the lack of intrinsic antibacterial activity, since efflux pumps are not vital for the organism's survival [151-152]. It was shown that this compound effectively potentiated the activity of levofloxacin, leading to an 8-fold decrease in the antibiotic's MIC. However, its physicochemical properties were problematic: water solubility was poor and it presented high affinity to serum albumin. Therefore, they divided the molecule into three parts, A, B, and C (137) and looked for alternative scaffolds and substituted polar groups, in order to reduce serum protein binding [152].

It was shown that when a carboxamide was introduced in A, the affinity to serum albumin was diminished, as was its activity. More polar moieties led to a complete loss of activity. The strategy for the styrene portion (B) that was the most successful was to substitute the benzene ring to a 3,5disubstituted pyridine, which had both good activity and no major serum influence. As for the vinyl group in B, it was demonstrated that molecules bearing methyloxy and ethylene moieties displayed favorable activity profiles. As for part C, the basis of the modifications was the fact that the introduction of bulky or hydrophilic groups adjacent to the carboxyl group would reduce protein binding. An enantiomeric pair containing a dioxolane moiety presented both activity and serum stability, being the $S$ enantiomer more active [152].

Regarding the general scaffold, new compounds were designed, keeping in mind that the thiazole moiety was crucial for activity. Therefore, a molecule having a thiazole side chain attached to the $\mathrm{C}-7$ position of a quinolone was synthesized. Substitution with a tetrazole portion yielded better results than substitution with a carboxylic acid. One compound (138) presented the best activity and serum stability [153].

Further efforts were placed in order to increase the potency of this compound. The in vitro activity was enhanced by the introduction of a hydrophobic group at the 2-position of the pyridopyrimidine scaffold, and hydrophilic substitution would not compromise the activity. The most promising compound had the inclusion of an olefin spacer between the tetrazole and the pyridopyrimidine scaffold, with the limitation of being prone to photoisomerization [154]. To circumvent this, the ethylene tether between the pyridopyrimidine was replaced with an amide bond, 
providing stability against photoisomerization and, unexpectedly, improving potency. However, another problem arose: the lack of solubility for intravenous use [155]. The following step was the introduction of a secondary amine, giving a zwitterionic analogue, with retention of activity. C-2 Analogues, namely with cyclic substituents, also showed promising results [156]. Once again, the problem of solubility arose. Therefore, efforts were placed in making the molecule more hydrophilic, with the introduction of substituted aromatic moieties. Analogues 4-substituted were the most potent, and the introduction of a morpholine moiety led to increased solubility, maintaining the serum stability. Compound $\mathbf{1 3 9}$ was the most balanced molecule in terms of the desired features [157]. The last refinement made was the introduction of a quaternary ammonium salt, which led to D13-9001 (140). Adding to its high solubility and safety, it was able to potentiate the activity of aztreonam [158]. Figure $\mathbf{1 5}$ illustrates the SAR established for 140 and derivatives.

3D Crystal structures of 140 bound to the target, either AcrB or MexB, show that the tert-butyl thiazolyl aminocarboxyl pyridopyrimidine moiety of this compound binds tightly to a narrow depression, designated by hydrophobic trap, close to the deep substrate binding pocket. Additionally, the tetrazole ring and the piperidine acetoamino ethylene ammonioacetate moiety interact with ionic and/or hydrophilic residues in the substrate translocation channel. The piperidine acetoamino ethylene ammonio-acetate moiety also covers part of the minocycline and doxorubicin binding site $[23,28$, 151].

Quinoline derivatives with EPI activity include a multiplicity of derivatives, including pyrrido-, alkoxy-, thioalkoxy-, alkylamino- and chloro- derivatives. These compounds were able to restore an intracellular concentration of antibiotic drugs expelled by efflux pumps, as well as to induce the increase in antibiotic susceptibility, acting as competitive inhibitors of the antibiotic flux. In $E$. aerogenes and $K$. pneumoniae, quinolines were more effective than $\mathrm{PA} \beta \mathrm{N}$ (124) in inhibiting the activity of the AcrB pump, potentiating the activity of fluoroquinolones, tetracycline, and chloramphenicol $[11,24,26]$. Among the different classes of substituted quinolines, it was proven that the best side chain was piperidinoethyl, which potentiated efficiently the restoration of drug susceptibility in alkoxy- and thioalkoxyquinolines. The heteroatom connecting the side chain is also crucial for activity, being the most potent the amino substituent, then the thioalkyl-, followed by the oxoderivative. The position of the branched substituted groups is also detrimental for the EPI activity [24]. Branched side chains containing piperidinoethyl and morpholinopropyl groups were associated with the most interesting results, being compound 141, containing a piperidinoethyl side chain, the most active when in combination with chloramphenicol [159]. It has been hypothesized that the pump inhibition is greatly influenced by the branched side chain, and takes place either in the inner-membrane transporter or at the junction between the inner pump and the outer channel [159].
Given these promising results, further research in this kind of compounds was performed. Alkoxyquinolines were synthesized and tested against a strain of $E$. aerogenes overexpressing the AcrAB efflux pump. Compound 142 induced an increase in the susceptibility of chloramphenicol, tetracycline, and fluoroquinolone. This could happen due to interference during active pumping out of the antibiotic [160].

Chloroquinolines also demonstrated efficacy as EPIs, modulating chloramphenicol activity, decreasing its MIC 8fold for resistant strains. Synthesis of analogues led to the conclusion that amino derivatives are more potent than thiol derivatives, pointing out the importance of the side chain for the efflux inhibition. Protonation of the nitrogen could also play an important role at environmental $\mathrm{pH}$, stimulating recognition and binding of the drug to the sites located within the pump's cavity. The heteroatom is also crucial for the activity, being nitrogen the most efficient. Compound 143 is the chloroquinoline with the best results found for the inhibition of the main efflux pump of E. aerogenes [161]. Alkylaminoquinazoline derivatives lacking a nitro group were also evaluated for their ability to decrease chloramphenicol and quinolone efflux in E. aerogenes, through the inhibition of AcrAB efflux pump. The results obtained suggest that this family of compounds recognizes the same pump site responsible for the transport of chloramphenicol and quinolones, competing with them. Structurally, it has been shown that a morpholine group with a propyl chain makes a more active alkylaminoquinazoline. The most active compound of this family is compound 144 [162].

Benzothiazoles 2-substituted were first tested for the potentiation of ciprofloxacin in AdeABC overexpressing $A$. baumanii. These compounds did not show antibacterial activity when tested alone in bacteria. However, when combined with ciprofloxacin, there was an observation of a reversal in antibacterial susceptibility. The synthesis of several derivatives of this family led to pharmacophore studies, regarding the features of the most promising compounds. Using computational methods, it was revealed that, in order for the compounds to be biologically active, the following features should be present: the nitrogen atom in the thiazole ring and the carbonyl oxygen in the amide function substituted on the second position of the benzothiazole ring must have an hydrogen bonding acceptor property; the benzene ring in the fused ring system, the phenyl group attached to the second position of the benzothiazole ring and the phenyl ring in the 2phenylacetamide and/or 3-phenylpropionamide moiety are essential, since they provide a hydrophobic aromatic property. Of the synthesized compounds, the ones that gathered these features and, therefore, presented the most promising activity, were compounds 145, 146, and 147 [163]. These compounds were also tested for their activity against AcrAB-TolC, in an overexpressing strain of E. coli, reducing the MIC of ciprofloxacin 10-fold, in the case of 146 and 147, and 8-fold, for 145 [164]. Unlike AdeABC, AcrAB-TolC's crystal structure is elucidated, making 
docking studies possible. This predicted that all three compounds act as AcrB substrates, binding to the distal pocket site in the AcrB porter domain by blocking or inhibiting the ciprofloxacin binding site, generating steric hindrance and thus impeding the binding of the antibiotic. Furthermore, 146 and 147 showed stronger binding interactions than ciprofloxacin [164].

Indole derivatives have been useful in inhibiting protein targets of virus, tumors and bacteria. Since small molecules with conjugated aromatic rings, in which indoles fit, present high potential as EPIs, efforts were placed in synthesizing derivatives that presented this kind of activity. The TolC 3D structure has already been disclosed, making possible the structure-based design of inhibitors. Indoles 148 and 149 were proven efficient inhibitors of TolC in $E$. coli, potentiating the effects of chloramphenicol, tetracycline, erythromycin, and ciprofloxacin, with decreased MIC values of 2-fold for tetracycline and erythromycin and 8-fold for the other two [165].

\subsubsection{Existing Drugs}

Other drugs used for treatments other than antibacterial have demonstrated EPI activity. Tetracycline resistance in $P$. aeruginosa was reduced 8-fold when the phenothiazine fluphenazine, an antipsychotic, was present. Chlorpromazine (150) also showed EPI-like activity for the BpeAB-OprB system in Burkholderia pseudomallei and a reduction of the expression of $a c r B$ in $E$. coli and $S$. enterica samovar Typhimurium, suggesting an inhibition of AcrB production $[11,27,128]$.

Selective serotonin-reuptake inhibitors have been studied for their ability to act as EPIs for RND efflux pumps in E. coli. It was found that sertraline (151) was capable of potentiating the activity of fluoroquinolones. However, its activity is limited, since it is possible that this compound also acts as an inductor of efflux pumps. Studies have shown that this compound could accumulate in tissues or cells, and therefore, sertraline's (151) concentration may be higher than that measured in plasma. This means that a standard dose of this drug could enhance antibiotic efficacy. It has not yet been studied if a sertraline (151) metabolite could be administered at a dose higher than sertraline. However, it was hypothesized that it could have a better EPI activity, while causing less undesired effects on the central nervous system [166].

Through the observation of previously reported EPIs, Piddock et al. (2010) suggested that simple heterocyclic nitrogen-containing compounds could also act as EPIs and synergize with ciprofloxacin in strains of $S$. enterica serovar Typhimurium that overexpressed the AcrAB-TolC efflux pump [167]. It has been shown that compounds containing a phenylethylamine moiety, as PA $\beta N$ (126) does, such as epinephrine, norepinephrine, and cathinone, are able to potentiate the activity of ciprofloxacin. Of the compounds tested, trimethoprim (152) and epinephrine (153), showed the best results for the tested strains, which included, apart from $S$. enterica serovar Typhimurium, P. aeruginosa, E. coli, and $K$. pneumoniae. These compounds showed synergy for the strains where AcrAB-TolC was active, but showed no activity when the pump was inactive. Thus, it can be hypothesized that these compounds either inhibit the expression of the genes encoding AcrAB-TolC, or interact directly with the pump [167]. Both compounds possess structural features similar to previously described EPIs, such as PA $\beta \mathrm{N}$ (124), L-Orn-L-hPhe-3-NHQ (125), and NMP (136), such as an aromatic ring linked to a basic nitrogen, in the case of epinephrine (153), or a pyrimidine ring, for trimethoprim (152). Trimethoprim (152) is already used in combination with another antibiotic, sulfamethoxazole, and combination with a quinolone was hypothesized to be a viable choice. On the other hand, a combination of epinephrine (153) with an antibiotic does not seem a viable option, because of the likelihood of the occurrence of sympathomimetic effects [167].

The antimalarial artesunate (154) has also shown EPI activity, enhancing the effects of $\beta$-lactam antibiotics, such as penicillin $\mathrm{G}$, oxacillin, and ampicillin, and novobiocin against $E$. coli. The mechanism through which 154 expresses its activity is by the inhibition of the expression of the AcrAB-TolC system, not exhibiting antibiotic activity by itself [168].

\section{SMR}

The SMR family comprises the smallest drug efflux proteins known, possessing only four transmembrane segments in its composition, and are exclusive to bacteria. They are involved in the efflux of lipophilic compounds, such as quaternary ammonium salts and a variety of antibiotics. These pumps also use an electrochemical proton gradient in order to exert its efflux activity [7, 32, 38, 169].

One example of a SMR-type pump is the EmrE transporter (Figure 16, PDB 3B61), present in E. coli. In the absence of the ligand, the asymmetric unit of EmrE contains eight EmrE monomers, with four transmembrane helices composing each of them. If a ligand is present, it can function as an inverted homodimer [7, 170].

There have not been many studies concerning inhibitors of this family of efflux pumps, and the few that have been performed were only in silico. Figure 17 shows the structures of the SMR inhibitors that will be further discussed.

\subsection{SMR inhibitors}

\subsubsection{Natural Products}

Quercetin (155) is a flavonol, present in many plants. This compound is very well tolerated in humans, as are its glycoconjugates, and presents a multiplicity of activities, such as antioxidant, anti-inflammatory and antimycobacterial. Only docking studies of this compound with two pumps belonging to the SMR family, the Mmr, in Mycobacterium smegmatis, and the EmrE pumps, in E. coli, were carried out. In Mmr, the hydroxyl groups present in the 
3' and 4' positions of the B ring of quercetin were predicted to interact with Glu-14 in the helix 1, and the keto group of the hydroxychromen ring of the ligand interacts with the Trp-48 of the helix 1. Additionally, hydrophobic interactions were shown by the residues Phe-44, Tyr-40 and Trp-48, and $\pi-\pi$ interactions were found with Phe- 44 . Furthermore, 155 was also predicted of interacting with dimeric forms of the protein [171]. In the case of EmrE, the interaction between the 3' and 4' hydroxyls with Glu-14 can also be observed, as can the hydroxyl in the C-7 position with Tyr-60. Hydrophobic interactions are present, with the residues Ile11, Ser-64, Gly-67 and Leu-70, and $\pi-\pi$ interactions exist with the residues Tyr-60 and Trp-63. Structural studies suggest that EmrE is mostly in the dimer state, which also interacts with quercetin (155) [171].

\subsubsection{Synthetic Compounds}

Cation ligands were subjected to docking studies with the Mmr protein of the SMR family in Mycobacterium tuberculosis and the EmrE dimer from E. coli. The rationale behind this study is the fact that SMR can extrude cations. This study led to the identification of new virtual leads, amongst which is compound 156. ADME studies prove that all the molecules present in this study obey the Lipinski rule of five and the Jorgenson rule of three, which shows that the compounds possess good drug-like properties [172].

\section{MATE}

MATE transporters are responsible for the efflux of cationic, lipophilic substances, providing bacteria and cancer cells with MDR, using $\mathrm{Na}^{+}$or $\mathrm{H}^{+}$influx. They have 12 transmembrane helices, constituted by 400 to 700 amino acids. The mechanism of efflux is thought to happen by a rocker-switch mechanism. Members of the MATE family include the MepA (Figure 18, PDB 1TZP) transporter, in $S$. aureus, even though seventeen MATE proteins have been identified in eleven species, capable of extruding antibiotics. Ethidium bromide, tetraphenylphosphonium, acriflavine, berberine, and norfloxacin have been reported as MATE substrates [38, 173-181].

In Figure 19 are compiled the structures of the compounds that have so far proven their usefulness as MATE inhibitors.

\subsection{MATE inhibitors}

\subsubsection{Natural Products}

A study performed by Kesherwani et al. (2017) accomplished the docking of phytochemicals with the NorM transporter, belonging to the MATE family [182]. After a screening of a library of natural products, three hits were identified: derhamnosyl suspenaside (157), prunin 7' $-O$ gallate (158), and quercetin diglucoside (159). These were the best compounds, as their results in binding free energy calculation were the most favorable. When binding free energy decomposition and hydrogen and hydrophobic statistics were taken into account, 158 was elected the best hit compound. Furthermore, this compound was stable during the simulation. Overall, it has been suggested that the compounds which interact towards the cation binding residue in the central cavity of NorM will have better results, and that good inhibitors should have interaction with a few key residues, Gln-34, Val-35, Gly-38, Phe-63, Tyr-67, Ile68, Glu-261, Tyr-294, and Asp-377, which will lead to maximum stability and affinity with the NorM transporter [182].

\subsubsection{Synthetic Compounds}

These compounds have been previously described as MFS inhibitors, namely of the NorA pump. However, similar activity has been described for the MepA pump, of the MATE family. Compound 80 (Figure 5), incorporating a bisaryl urea moiety at the 7-position, was able to successfully inhibit approximately $84 \%$ of ethidium bromide efflux in a MepA overexpressing strain of S.aureus [104]. Phenylquinoline derivatives also proved efficient against the same strain. Differently from what was observed with NorA, the compounds that inhibited MepA most efficiently were 86 (Figure 5) and 160 (Figure 19), which were able to reduce ethidium bromide's MIC by 16-fold at concentrations of 0.78 and $1.56 \mu \mathrm{g} / \mathrm{ml}$, respectively, in the MepA overexpressing strain. Ethidium bromide was chosen, instead of ciprofloxacin, as it is a better MepA substrate and other derivatives were able to inhibit both NorA and MepA efflux pumps, being able to completely restore the activity of ciprofloxacin and ethidium bromide against strains overexpressing such pumps [107].

Paroxetine derivatives (104), effective against the NorA pump, also demonstrated potency in inhibiting the MepA pump in $S$. aureus. Oppositely to NorA, paroxetine derivatives do not need the fluorophenyl ring at position 4 to be MepA EPIs. In fact, 4-unsubstituted analogues proved to be more potent than 4-F-phenyl derivatives in inhibiting the MepA pump, as is the case of compound 161 and 162 [122].

Efflux pumps are one of the main causes for antimicrobial resistance. Their inhibition can lead to the restoration of bacteria susceptibility and antibiotic efficacy. Throughout this review, the data presented leads to the conclusion that EPIs are promising compounds and can help reversing the problem of antimicrobial resistance. However, the research into these kind of compounds is still in a very preliminary state, since no EPI is yet under clinical trials.

Even though no general structural features can be pointed out for a molecule to be an EPI, it can be observed that small molecules with high lipophilicity are most likely to fulfill the requirements to inhibit efflux pumps. Hydrophilic compounds can also be transported through porins present in the membrane. Heterocyclic compounds also show potential as EPIs, mainly nitrogen heterocycles, but also fluorine and boron heteroatoms, since the likelihood of bacterial exposure to these atoms is very low. The basis for the MDR pumps inhibition has to do with the penetration rules, and whether 
or not a compound is capable of penetrating the bacterial membrane. Therefore, a library of compounds that are able to do that would be something that would bring advances to this study field.

One of the main aims of this review is to present SAR studies, so that pharmaceutical chemists can refine molecular structures, leading to the achievement of more potent and specific compounds. The use of synthetic approaches, either to modify molecules obtained from natural sources, or drugs that are already in therapeutic use, or even to develop novel compounds, is vital for these SAR studies. The search of EPIs derived from natural products comes from the fact that plants need to protect themselves from pathogenic microorganisms, and produce metabolites that act as antimicrobial and EPIs, that synergize with each other. However, the referred compounds are not specific for the pump they have been studied, showing a multitarget character: flavonoids, coumarins, cinnamic acids, among others, present a multiplicity of activities already described, suggesting their promiscuity.

The advantage of assays with existing drugs lies in their safety profile and, most times, their clinical use for combination therapy has been characterized. In some cases, the compounds were even investigated as inhibitors of human efflux pumps, for cancer treatment. This can be regarded as an advantage, leading to a new class of drugs with dual human/bacterial EPI, particularly useful in cancer patients with infections, potentiating the effects of both the anticancer and the antibacterial drugs.

The future in studies in bacterial EPIs should lie either in the search for selectivity for the bacterial target, or in the disclosure of the common features of the universal bacterial EPI, that can successfully target all efflux pumps. The SAR studies gathered herein with the $3 \mathrm{D}$ structural information available are expected to accelerate the structure-based design of new EPIs, founded on the existing models.

\section{LIST OF ABBREVIATIONS}

3D-QSAR: Three-dimensional quantitative structure activity relationship; ABC: ATP-binding cassette; ADME: Absorption, distribution, metabolism and excretion; ATP: Adenosine triphosphate; BCRP: Breast cancer resistance protein; COX-2: Ciclooxigenase-2; CYP450: Cytochrome P450; EPI: Efflux pump inhibitor; $\mathrm{IC}_{50}$ : Half maximal inhibitory concentration; MATE: Multidrug and toxic compound extrusion; MDR: Multidrug resistance; MIC: Minimum inhibitory concentration; MFS: Major facilitator superfamily; MRP: Multidrug resistance protein; MRSA: Methicillin-resistant Staphylococcus aureus; NDGA: Nordihydroguaretic acid; NMP: 1-(1-Naphthylmethyl)piperazine; P-gp: P-Glycoprotein; PA $\beta \mathrm{N}$ : Phenyl-arginine $\beta$ naphtylamide; PDB: Protein Data Bank; PSSRI: Phenylpiperidine selective serotonin reuptake inhibitors; RND: Resistance-nodulation-division; SAR: Structure activity relationship; SMR: Small multidrug resistance.

\section{ACKNOWLEDGEMENTS}

This work was supported through national funds provided by FCT/MCTES - Foundation for Science and Technology from the Minister of Science, Technology and Higher Education (PIDDAC) and European Regional Development Fund (ERDF) through the COMPETE - Programa Operacional Factores de Competitividade (POFC) programme, under the project PTDC/MAR-BIO/4694/2014 (reference POCI-010145-FEDER-016790; Project 3599 - Promover a Produção Científica e Desenvolvimento Tecnológico e a Constituição de Redes Temáticas (3599-PPCDT)) and PTDC/AAGTEC/0739/2014 (POCI-01-0145-FEDER-016793, Projeto 9471) in the framework of the programme PT2020 and by the project INNOVMAR - Innovation and Sustainability in the Management and Exploitation of Marine Resources (reference NORTE-01-0145-FEDER-000035, within Research Line NOVELMAR), supported by North Portugal Regional Operational Programme (NORTE 2020), under the PORTUGAL 2020 Partnership Agreement, through the ERDF and was partially supported by the Strategic Funding UID/Multi/04423/2013 through national funds provided by FCT, and ERDF, in the framework of the programme PT2020.

\section{REFERENCES}

[1] Davies, J.; Davies, D., Origins and Evolution of Antibiotic Resistance. Microbiol Mol Biol Rev 2010, 74 (3), 417-433.

[2] Alanis, A. J., Resistance to antibiotics: are we in the postantibiotic era? Arch Med Res 2005, 36 (6), 697-705.

[3] Ventola, C. L., The Antibiotic Resistance Crisis: Part 1: Causes and Threats. P T 2015, 40 (4), 277-283.

[4] Levy, S. B.; Marshall, B., Antibacterial resistance worldwide: causes, challenges and responses. Nat Med 2004, 10 (12 Suppl), S122-9.

[5] Munita, J. M.; Arias, C. A., Mechanisms of Antibiotic Resistance. Microbiol Spect 2016, 4 (2), 10.1128/microbiolspec.VMBF-0016-2015.

[6] Chattopadhyay, M. K.; Jagannadham, M. V., Vesiclesmediated resistance to antibiotics in bacteria. FrontMicrobiol 2015, 6,758 .

[7] Sun, J.; Deng, Z.; Yan, A., Bacterial multidrug efflux pumps: mechanisms, physiology and pharmacological exploitations. Biochem Biophys Res Commun 2014, 453 (2), 254-67.

[8] Blanco, P.; Hernando-Amado, S.; Reales-Calderon, J. A.; Corona, F.; Lira, F.; Alcalde-Rico, M.; Bernardini, A.; Sanchez, M. B.; Martinez, J. L., Bacterial Multidrug Efflux Pumps: Much More Than Antibiotic Resistance Determinants. Microorganisms 2016, 4 (1).

[9] Fernandez, L.; Hancock, R. E., Adaptive and mutational resistance: role of porins and efflux pumps in drug resistance. Clin Microbiol Rev 2012, 25 (4), 661-81.

[10] Webber, M. A.; Piddock, L. J. V., The importance of efflux pumps in bacterial antibiotic resistance. J Antimicrob Chemother 2003, 51 (1), 9-11. 
[11] Tegos, G. P.; Haynes, M.; Strouse, J. J.; Khan, M. M.; Bologa, C. G.; Oprea, T. I.; Sklar, L. A., Microbial efflux pump inhibition: tactics and strategies. Curr Pharm Des 2011, 17 (13), 1291-302.

[12] Van Bambeke, F.; Pages, J. M.; Lee, V. J., Inhibitors of bacterial efflux pumps as adjuvants in antibiotic treatments and diagnostic tools for detection of resistance by efflux. Recent Pat Antiinfect Drug Discov 2006, 1 (2), 157-75.

[13] Marquez, B., Bacterial efflux systems and efflux pumps inhibitors. Biochimie 2005, 87 (12), 1137-47.

[14] Kumar, R.; Patial, S., A Review on Efflux Pump Inhibitors of Gram-Positive and Gram-Negative Bacteria from Plant Sources. Int J Curr Microbiol App Sci 2016, 5 (6), 834-855.

[15] Rana, T.; Singh, S.; Kaur, N.; Pathania, K.; Farooq, U., A Review on Efflux Pump Inhibitors of Medically Important Bacteria from Plant Sources. Int J Pharm Sci Rev Res 2014, 26 (2), 101-111.

[16] Prasch, S.; Bucar, F., Plant derived inhibitors of bacterial efflux pumps: an update. Phytochem Rev 2015, 14 (6), 961-974.

[17] Zhang, L.; Ma, S., Efflux pump inhibitors: a strategy to combat P-glycoprotein and the NorA multidrug resistance pump. Chem Med Chem 2010, 5 (6), 811-22.

[18] Schindler, B. D.; Jacinto, P.; Kaatz, G. W., Inhibition of drug efflux pumps in Staphylococcus aureus: current status of potentiating existing antibiotics. Future Microbiol 2013, 8 (4), 491-507.

[19] Kumar, S.; Mukherjee, M. M.; Varela, M. F., Modulation of Bacterial Multidrug Resistance Efflux Pumps of the Major Facilitator Superfamily. Int J Bacteriol 2013, 2013.

[20] Handzlik, J.; Matys, A.; Kiec-Kononowicz, K., Recent Advances in Multi-Drug Resistance (MDR) Efflux Pump Inhibitors of Gram-Positive Bacteria S. aureus. Antibiotics (Basel) 2013, 2 (1), 28-45.

[21] Stavri, M.; Piddock, L. J. V.; Gibbons, S., Bacterial efflux pump inhibitors from natural sources. $J$ Antimicrob Chemother 2007, 59 (6), 1247-1260.

[22] Gibbons, S., Plants as a Source of Bacterial Resistance Modulators and Anti-Infective Agents. Phytochem Rev 2005, 4 (1), 63-78.

[23] Ruggerone, P.; Murakami, S.; Pos, K. M.; Vargiu, A. V., RND efflux pumps: structural information translated into function and inhibition mechanisms. Curr Top Med Chem 2013, 13 (24), 3079-100.

[24] Pagès, J.-M.; Masi, M.; Barbe, J., Inhibitors of efflux pumps in Gram-negative bacteria. Trends Mol Med 2005, 11 (8), 382-389.

[25] Opperman, T.; Nguyen, S., Recent advances toward a molecular mechanism of efflux pump inhibition. Front Microbiol 2015, 6 (421).

[26] Martins, M.; Dastidar, S. G.; Fanning, S.; Kristiansen, J. E.; Molnar, J.; Pages, J. M.; Schelz, Z.; Spengler, G.; Viveiros, M.; Amaral, L., Potential role of non-antibiotics (helper compounds) in the treatment of multidrug-resistant Gram-negative infections: mechanisms for their direct and indirect activities. Int J Antimicrob Agents 2008, 31 (3), 198208.

[27] Blair, J. M. A.; Piddock, L. J. V., Structure, function and inhibition of RND efflux pumps in Gram-negative bacteria: an update. Curr Opin Microbiol 2009, 12 (5), 512519.

[28] Aron, Z.; Opperman, T. J., Optimization of a novel series of pyranopyridine RND efflux pump inhibitors. Curr Opin Microbiol 2016, 33, 1-6.

[29] Venter, H.; Mowla, R.; Ohene-Agyei, T.; Ma, S., RNDtype drug efflux pumps from Gram-negative bacteria: molecular mechanism and inhibition. Front Microbiol 2015, 6,377 .

[30] Song, L.; Wu, X., Development of efflux pump inhibitors in antituberculosis therapy. Int $J$ Antimicrob Agents 2016, 47 (6), 421-9.

[31] Pule, C. M.; Sampson, S. L.; Warren, R. M.; Black, P. A.; van Helden, P. D.; Victor, T. C.; Louw, G. E., Efflux pump inhibitors: targeting mycobacterial efflux systems to enhance TB therapy. J Antimicrob Chemother 2016, 71 (1), 17-26.

[32] Zechini, B.; Versace, I., Inhibitors of multidrug resistant efflux systems in bacteria. Recent Pat Antiinfect Drug Discov 2009, 4 (1), 37-50.

[33] El-Awady, R.; Saleh, E.; Hashim, A.; Soliman, N.; Dallah, A.; Elrasheed, A.; Elakraa, G., The Role of Eukaryotic and Prokaryotic ABC Transporter Family in Failure of Chemotherapy. Front Pharmacol 2016, 7, 535.

[34] Silva, R.; Vilas-Boas, V.; Carmo, H.; Dinis-Oliveira, R. J.; Carvalho, F.; de Lourdes Bastos, M.; Remião, F., Modulation of P-glycoprotein efflux pump: induction and activation as a therapeutic strategy. Pharmacol Ther 2015, 149, 1-123.

[35] Palmeira, A.; Rodrigues, F.; Sousa, E.; Pinto, M.; Vasconcelos, M. H.; Fernandes, M. X., New uses for old drugs: pharmacophore-based screening for the discovery of P-glycoprotein inhibitors. Chem Biol Drug Des 2011, 78 (1), 57-72.

[36] Lopes-Rodrigues, V.; Sousa, E.; Vasconcelos, M. H., Curcumin as a Modulator of P-Glycoprotein in Cancer: Challenges and Perspectives. Pharmaceuticals (Basel) 2016, 9 (4).

[37] Lopes-Rodrigues, V.; Seca, H.; Sousa, D.; Sousa, E.; Lima, R. T.; Vasconcelos, M. H., The network of Pglycoprotein and microRNAs interactions. Int $J$ Cancer 2014, 135 (2), 253-63.

[38] Jang, S., Multidrug efflux pumps in Staphylococcus aureus and their clinical implications. J Microbiol 2016, 54 (1), 1-8.

[39] Locher, K. P., Mechanistic diversity in ATP-binding cassette (ABC) transporters. Nat Struct Mol Biol 2016, 23 (6), 487-93.

[40] Wilkens, S., Structure and mechanism of ABC transporters. F1000Prime Rep 2015, 7, 14.

[41] Taylor, A. B.; Benglis, J. D. M.; Dhandayuthapani, S.; Hart, P. J., Structure of Mycobacterium tuberculosis Methionine Sulfoxide Reductase A in Complex with ProteinBound Methionine. J Bacteriol 2003, 185 (14), 4119-4126. 
[42] Oluwatuyi, M.; Kaatz, G. W.; Gibbons, S., Antibacterial and resistance modifying activity of Rosmarinus officinalis. Phytochemistry 2004, 65 (24), 3249-54.

[43] Hussein, A. A.; Rodriguez, B., Isopimarane diterpenoids from Lycopus europaeus. J Nat Prod 2000, 63 (3), 419-21.

[44] Gibbons, S.; Oluwatuyi, M.; Veitch, N. C.; Gray, A. I., Bacterial resistance modifying agents from Lycopus europaeus. Phytochemistry 2003, 62 (1), 83-7.

[45] Upadhyay, H. C.; Dwivedi, G. R.; Roy, S.; Sharma, A.; Darokar, M. P.; Srivastava, S. K., Phytol derivatives as drug resistance reversal agents. Chem Med Chem 2014, 9 (8), 1860-8

[46] Dwivedi, G. R.; Maurya, A.; Yadav, D. K.; Khan, F.; Darokar, M. P.; Srivastava, S. K., Drug Resistance Reversal Potential of Ursolic Acid Derivatives against Nalidixic Acidand Multidrug-resistant Escherichia coli. Chem Biol Drug Des 2015, 86 (3), 272-83.

[47] Wang, S. Y.; Sun, Z. L.; Liu, T.; Gibbons, S.; Zhang, W. J.; Qing, M., Flavonoids from Sophora moorcroftiana and their synergistic antibacterial effects on MRSA. Phytother Res 2014, 28 (7), 1071-6.

[48] Chan, B. C.; Ip, M.; Gong, H.; Lui, S. L.; See, R. H.; Jolivalt, C.; Fung, K. P.; Leung, P. C.; Reiner, N. E.; Lau, C. B., Synergistic effects of diosmetin with erythromycin against ABC transporter over-expressed methicillin-resistant Staphylococcus aureus (MRSA) RN4220/pUL5054 and inhibition of MRSA pyruvate kinase. Phytomedicine 2013, 20 (7), 611-4.

[49] Dwivedi, G. R.; Upadhyay, H. C.; Yadav, D. K.; Singh, V.; Srivastava, S. K.; Khan, F.; Darmwal, N. S.; Darokar, M. P., 4-Hydroxy- $\alpha$-tetralone and its derivative as drug resistance reversal agents in multi drug resistant Escherichia coli. Chem Biol Drug Des 2014, 83 (4), 482-92.

[50] Chan, B. C.; Han, X. Q.; Lui, S. L.; Wong, C. W.; Wang, T. B.; Cheung, D. W.; Cheng, S. W.; Ip, M.; Han, S. Q.; Yang, X. S.; Jolivalt, C.; Lau, C. B.; Leung, P. C.; Fung, K. P., Combating against methicillin-resistant Staphylococcus aureus - two fatty acids from Purslane (Portulaca oleracea L.) exhibit synergistic effects with erythromycin. J Pharm Pharmacol 2015, 67 (1), 107-16.

[51] Maurya, A.; Dwivedi, G. R.; Darokar, M. P.; Srivastava, S. K., Antibacterial and synergy of clavine alkaloid lysergol and its derivatives against nalidixic acid-resistant Escherichia coli. Chem Biol Drug Des 2013, 81 (4), 484-90. [52] Gibbons, S.; Oluwatuyi, M.; Kaatz, G. W., A novel inhibitor of multidrug efflux pumps in Staphylococcus aureus. J Antimicrob Chemother 2003, 51 (1), 13-7.

[53] Pezza, R. J.; Villarreal, M. A.; Montich, G. G.; Argaraña, C. E., Vanadate inhibits the ATPase activity and DNA binding capability of bacterial MutS. A structural model for the vanadate-MutS interaction at the Walker A motif. Nucleic Acids Res 2002, 30 (21), 4700-4708.

[54] Yan, N., Structural advances for the major facilitator superfamily (MFS) transporters. Trends in Biochem Sci 2013, 38 (3), 151-159.

[55] Yin, Y.; He, X.; Szewczyk, P.; Nguyen, T.; Chang, G., Structure of the Multidrug Transporter EmrD from
Escherichia coli. Science (New York, N.Y.) 2006, 312 (5774), 741-744.

[56] Yan, N., Structural Biology of the Major Facilitator Superfamily Transporters. Annu Rev Biophys 2015, 44, 257 83

[57] Tamura, N.; Konishi, S.; Yamaguchi, A., Mechanisms of drug/H+ antiport: complete cysteine-scanning mutagenesis and the protein engineering approach. Curr Opin Chem Biol 2003, 7 (5), 570-579.

[58] Coelho, M. L.; Ferreira, J. H.; de Siqueira Junior, J. P.; Kaatz, G. W.; Barreto, H. M.; de Carvalho Melo Cavalcante, A. A., Inhibition of the NorA multi-drug transporter by oxygenated monoterpenes. Microb Pathog 2016, 99, 173177.

[59] Thota, N.; Koul, S.; Reddy, M. V.; Sangwan, P. L.; Khan, I. A.; Kumar, A.; Raja, A. F.; Andotra, S. S.; Qazi, G. N., Citral derived amides as potent bacterial NorA efflux pump inhibitors. Bioorg Med Chem 2008, 16 (13), 6535-43. [60] Smith, E. C.; Williamson, E. M.; Wareham, N.; Kaatz, G. W.; Gibbons, S., Antibacterials and modulators of bacterial resistance from the immature cones of Chamaecyparis lawsoniana. Phytochemistry 2007, 68 (2), 210-7.

[61] Smith, E. C.; Kaatz, G. W.; Seo, S. M.; Wareham, N.; Williamson, E. M.; Gibbons, S., The phenolic diterpene totarol inhibits multidrug efflux pump activity in Staphylococcus aureus. Antimicrob Agents Chemother 2007, 51 (12), 4480-3.

[62] Zhang, J.; Sun, Y.; Wang, Y.; Lu, M.; He, J.; Liu, J.; Chen, Q.; Zhang, X.; Zhou, F.; Wang, G.; Sun, X., Nonantibiotic agent ginsenoside $20(S)-\mathrm{Rh} 2$ enhanced the antibacterial effects of ciprofloxacin in vitro and in vivo as a potential NorA inhibitor. Eur J Pharmacol 2014, 740, 277 84.

[63] Zhou, S.; Schuetz, J. D.; Bunting, K. D.; Colapietro, A. M.; Sampath, J.; Morris, J. J.; Lagutina, I.; Grosveld, G. C.; Osawa, M.; Nakauchi, H.; Sorrentino, B. P., The ABC transporter Bcrp1/ABCG2 is expressed in a wide variety of stem cells and is a molecular determinant of the sidepopulation phenotype. Nat Med 2001, 7 (9), 1028-34.

[64] Doyle, L.; Ross, D. D., Multidrug resistance mediated by the breast cancer resistance protein BCRP (ABCG2). Oncogene 2003, 22 (47), 7340-58.

[65] Ahmed, M.; Borsch, C. M.; Neyfakh, A. A.; Schuldiner, S., Mutants of the Bacillus subtilis multidrug transporter Bmr with altered sensitivity to the antihypertensive alkaloid reserpine. J Biol Chem 1993, 268 (15), 11086-9.

[66] Schmitz, F. J.; Fluit, A. C.; Luckefahr, M.; Engler, B.; Hofmann, B.; Verhoef, J.; Heinz, H. P.; Hadding, U.; Jones, M. E., The effect of reserpine, an inhibitor of multidrug efflux pumps, on the in vitro activities of ciprofloxacin, sparfloxacin and moxifloxacin against clinical isolates of Staphylococcus aureus. J Antimicrob Chemother 1998, 42 (6), 807-10.

[67] Markham, P. N.; Westhaus, E.; Klyachko, K.; Johnson, M. E.; Neyfakh, A. A., Multiple Novel Inhibitors of the NorA Multidrug Transporter of Staphylococcus aureus. Antimicrob Agents Chemother 1999, 43 (10), 2404-8. 
[68] Kumar, A.; Khan, I. A.; Koul, S.; Koul, J. L.; Taneja, S. C.; Ali, I.; Ali, F.; Sharma, S.; Mirza, Z. M.; Kumar, M.; Sangwan, P. L.; Gupta, P.; Thota, N.; Qazi, G. N., Novel structural analogues of piperine as inhibitors of the NorA efflux pump of Staphylococcus aureus. $J$ Antimicrob Chemother 2008, 61 (6), 1270-6.

[69] Sangwan, P. L.; Koul, J. L.; Koul, S.; Reddy, M. V.; Thota, N.; Khan, I. A.; Kumar, A.; Kalia, N. P.; Qazi, G. N., Piperine analogs as potent Staphylococcus aureus NorA efflux pump inhibitors. Bioorg Med Chem 2008, 16 (22), 9847-57.

[70] Nargotra, A.; Sharma, S.; Koul, J. L.; Sangwan, P. L.; Khan, I. A.; Kumar, A.; Taneja, S. C.; Koul, S., Quantitative structure activity relationship (QSAR) of piperine analogs for bacterial NorA efflux pump inhibitors. Eur J Med Chem 2009, 44 (10), 4128-35.

[71] Stermitz, F. R.; Lorenz, P.; Tawara, J. N.; Zenewicz, L. A.; Lewis, K., Synergy in a medicinal plant: antimicrobial action of berberine potentiated by 5'-methoxyhydnocarpin, a multidrug pump inhibitor. Proc Natl Acad Sci U S A 2000, 97 (4), 1433-7.

[72] Guz, N. R.; Stermitz, F. R.; Johnson, J. B.; Beeson, T. D.; Willen, S.; Hsiang, J.; Lewis, K., Flavonolignan and flavone inhibitors of a Staphylococcus aureus multidrug resistance pump: structure-activity relationships. $J$ Med Chem 2001, 44 (2), 261-8.

[73] Bame, J. R.; Graf, T. N.; Junio, H. A.; Bussey, R. O., 3rd; Jarmusch, S. A.; El-Elimat, T.; Falkinham, J. O., 3rd; Oberlies, N. H.; Cech, R. A.; Cech, N. B., Sarothrin from Alkanna orientalis is an antimicrobial agent and efflux pump inhibitor. Planta Med 2013, 79 (5), 327-9.

[74] Morel, C.; Stermitz, F. R.; Tegos, G.; Lewis, K., Isoflavones as potentiators of antibacterial activity. J Agric Food Chem 2003, 51 (19), 5677-9.

[75] Chan, B. C.; Ip, M.; Lau, C. B.; Lui, S. L.; Jolivalt, C.; Ganem-Elbaz, C.; Litaudon, M.; Reiner, N. E.; Gong, H.; See, R. H.; Fung, K. P.; Leung, P. C., Synergistic effects of baicalein with ciprofloxacin against NorA over-expressed methicillin-resistant Staphylococcus aureus (MRSA) and inhibition of MRSA pyruvate kinase. J Ethnopharmacol 2011, 137 (1), 767-73.

[76] Maia, G. L.; Falcao-Silva Vdos, S.; Aquino, P. G.; de Araujo-Junior, J. X.; Tavares, J. F.; da Silva, M. S.; Rodrigues, L. C.; de Siqueira-Junior, J. P.; Barbosa-Filho, J. M., Flavonoids from Praxelis clematidea R.M. King and Robinson modulate bacterial drug resistance. Molecules 2011, 16 (6), 4828-35.

[77] Falcao-Silva, V. S.; Silva, D. A.; Souza Mde, F.; Siqueira-Junior, J. P., Modulation of drug resistance in Staphylococcus aureus by a kaempferol glycoside from Herissantia tiubae (Malvaceae). Phytother Res 2009, 23 (10), 1367-70.

[78] Holler, J. G.; Christensen, S. B.; Slotved, H. C.; Rasmussen, H. B.; Guzman, A.; Olsen, C. E.; Petersen, B.; Molgaard, P., Novel inhibitory activity of the Staphylococcus aureus NorA efflux pump by a kaempferol rhamnoside isolated from Persea lingue Nees. J Antimicrob Chemother 2012, 67 (5), 1138-44.
[79] Holler, J. G.; Slotved, H. C.; Molgaard, P.; Olsen, C. E.; Christensen, S. B., Chalcone inhibitors of the NorA efflux pump in Staphylococcus aureus whole cells and enriched everted membrane vesicles. Bioorg Med Chem 2012, 20 (14), 4514-21.

[80] Belofsky, G.; Percivill, D.; Lewis, K.; Tegos, G. P.; Ekart, J., Phenolic metabolites of Dalea versicolor that enhance antibiotic activity against model pathogenic bacteria. J Nat Prod 2004, 67 (3), 481-4.

[81] Stermitz, F. R.; Tawara-Matsuda, J.; Lorenz, P.; Mueller, P.; Zenewicz, L.; Lewis, K., 5'Methoxyhydnocarpin-D and pheophorbide A: Berberis species components that potentiate berberine growth inhibition of resistant Staphylococcus aureus. J Nat Prod 2000, 63 (8), 1146-9.

[82] Pereda-Miranda, R.; Kaatz, G. W.; Gibbons, S., Polyacylated oligosaccharides from medicinal Mexican morning glory species as antibacterials and inhibitors of multidrug resistance in Staphylococcus aureus. J Nat Prod 2006, 69 (3), 406-9.

[83] Cherigo, L.; Pereda-Miranda, R.; Fragoso-Serrano, M.; Jacobo-Herrera, N.; Kaatz, G. W.; Gibbons, S., Inhibitors of bacterial multidrug efflux pumps from the resin glycosides of Ipomoea murucoides. J Nat Prod 2008, 71 (6), 1037-45.

[84] Stermitz, F. R.; Cashman, K. K.; Halligan, K. M.; Morel, C.; Tegos, G. P.; Lewis, K., Polyacylated neohesperidosides From Geranium caespitosum: bacterial multidrug resistance pump inhibitors. Bioorg Med Chem Lett 2003, 13 (11), 1915-1918.

[85] Kalia, N. P.; Mahajan, P.; Mehra, R.; Nargotra, A.; Sharma, J. P.; Koul, S.; Khan, I. A., Capsaicin, a novel inhibitor of the NorA efflux pump, reduces the intracellular invasion of Staphylococcus aureus. J Antimicrob Chemother 2012, 67 (10), 2401-8.

[86] Fiamegos, Y. C.; Kastritis, P. L.; Exarchou, V.; Han, H.; Bonvin, A. M. J. J.; Vervoort, J.; Lewis, K.; Hamblin, M. R.; Tegos, G. P., Antimicrobial and Efflux Pump Inhibitory Activity of Caffeoylquinic Acids from Artemisia absinthium against Gram-Positive Pathogenic Bacteria. PLOS ONE 2011, 6 (4), e18127.

[87] Shiu, W. K.; Malkinson, J. P.; Rahman, M. M.; Curry, J.; Stapleton, P.; Gunaratnam, M.; Neidle, S.; Mushtaq, S.; Warner, M.; Livermore, D. M.; Evangelopoulos, D.; Basavannacharya, C.; Bhakta, S.; Schindler, B. D.; Seo, S. M.; Coleman, D.; Kaatz, G. W.; Gibbons, S., A new plantderived antibacterial is an inhibitor of efflux pumps in Staphylococcus aureus. Int J Antimicrob Agents 2013, 42 (6), 513-8.

[88] Costa, L. M.; de Macedo, E. V.; Oliveira, F. A.; Ferreira, J. H.; Gutierrez, S. J.; Pelaez, W. J.; Lima, F. C.; de Siqueira Junior, J. P.; Coutinho, H. D.; Kaatz, G. W.; de Freitas, R. M.; Barreto, H. M., Inhibition of the NorA efflux pump of Staphylococcus aureus by synthetic riparins. J Appl Microbiol 2016, 121 (5), 1312-1322.

[89] Michalet, S.; Cartier, G.; David, B.; Mariotte, A. M.; Dijoux-franca, M. G.; Kaatz, G. W.; Stavri, M.; Gibbons, S., $\mathrm{N}$-caffeoylphenalkylamide derivatives as bacterial efflux 
pump inhibitors. Bioorg Med Chem Lett 2007, 17 (6), 17558.

[90] Abulrob, A. N.; Suller, M. T.; Gumbleton, M.; Simons, C.; Russell, A. D., Identification and biological evaluation of grapefruit oil components as potential novel efflux pump modulators in methicillin-resistant Staphylococcus aureus bacterial strains. Phytochemistry 2004, 65 (22), 3021-7.

[91] Roy, S. K.; Kumari, N.; Pahwa, S.; Agrahari, U. C.; Bhutani, K. K.; Jachak, S. M.; Nandanwar, H., NorA efflux pump inhibitory activity of coumarins from Mesua ferrea. Fitoterapia 2013, 90, 140-50.

[92] Oliveira, A. S.; Sousa, E.; Vasconcelos, M. H.; Pinto, M., Curcumin: A Natural Lead for Potential New Drug Candidates. Curr Med Chem 2015, 22 (36), 4196-232.

[93] Lopes-Rodrigues, V.; Oliveira, A.; Correia-da-Silva, M.; Pinto, M.; Lima, R. T.; Sousa, E.; Vasconcelos, M. H., A novel curcumin derivative which inhibits P-glycoprotein, arrests cell cycle and induces apoptosis in multidrug resistance cells. Bioorg Med Chem 2017, 25 (2), 581-596.

[94] Joshi, P.; Singh, S.; Wani, A.; Sharma, S.; Jain, S. K.; Singh, B.; Gupta, B. D.; Satti, N. K.; Koul, S.; Khan, I. A.; Kumar, A.; Bharate, S. B.; Vishwakarma, R. A., Osthol and curcumin as inhibitors of human Pgp and multidrug efflux pumps of Staphylococcus aureus: reversing the resistance against frontline antibacterial drugs. Med Chem Comm 2014, 5 (10), 1540-1547.

[95] Marquez, B.; Neuville, L.; Moreau, N. J.; Genet, J. P.; dos Santos, A. F.; Cano de Andrade, M. C.; Sant'Ana, A. E., Multidrug resistance reversal agent from Jatropha elliptica. Phytochemistry 2005, 66 (15), 1804-11.

[96] Belofsky, G.; Carreno, R.; Lewis, K.; Ball, A.; Casadei, G.; Tegos, G. P., Metabolites of the "smoke tree", Dalea spinosa, potentiate antibiotic activity against multidrugresistant Staphylococcus aureus. J Nat Prod 2006, 69 (2), 261-4.

[97] Ambrus, J. I.; Kelso, M. J.; Bremner, J. B.; Ball, A. R.; Casadei, G.; Lewis, K., Structure-activity relationships of 2aryl-1H-indole inhibitors of the NorA efflux pump in Staphylococcus aureus. Bioorg Med Chem Lett 2008, 18 (15), 4294-7.

[98] Samosorn, S.; Bremner, J. B.; Ball, A.; Lewis, K., Synthesis of functionalized 2-aryl-5-nitro- $1 H$-indoles and their activity as bacterial NorA efflux pump inhibitors. Bioorg Med Chem 2006, 14 (3), 857-65.

[99] Ball, A. R.; Casadei, G.; Samosorn, S.; Bremner, J. B.; Ausubel, F. M.; Moy, T. I.; Lewis, K., Conjugating berberine to a multidrug efflux pump inhibitor creates an effective antimicrobial. ACS Chem Biol 2006, 1 (9), 594-600.

[100] Fournier dit Chabert, J.; Marquez, B.; Neville, L.; Joucla, L.; Broussous, S.; Bouhours, P.; David, E.; PelletRostaing, S.; Marquet, B.; Moreau, N.; Lemaire, M., Synthesis and evaluation of new arylbenzo[b]thiophene and diarylthiophene derivatives as inhibitors of the NorA multidrug transporter of Staphylococcus aureus. Bioorg Med Chem 2007, 15 (13), 4482-4497.

[101] Samosorn, S.; Tanwirat, B.; Muhamad, N.; Casadei, G.; Tomkiewicz, D.; Lewis, K.; Suksamrarn, A.; Prammananan, T.; Gornall, K. C.; Beck, J. L.; Bremner, J.
B., Antibacterial activity of berberine-NorA pump inhibitor hybrids with a methylene ether linking group. Bioorg Med Chem 2009, 17 (11), 3866-72.

[102] Buonerba, F.; Lepri, S.; Goracci, L.; Schindler, B. D.; Seo, S. M.; Kaatz, G. W.; Cruciani, G., Improved Potency of Indole-Based NorA Efflux Pump Inhibitors: From Serendipity toward Rational Design and Development. $J$ Med Chem 2017, 60 (1), 517-523.

[103] Lepri, S.; Buonerba, F.; Goracci, L.; Velilla, I.; Ruzziconi, R.; Schindler, B. D.; Seo, S. M.; Kaatz, G. W.; Cruciani, G., Indole Based Weapons to Fight Antibiotic Resistance: A Structure-Activity Relationship Study. J Med Chem 2016, 59 (3), 867-91.

[104] German, N.; Wei, P.; Kaatz, G. W.; Kerns, R. J., Synthesis and evaluation of fluoroquinolone derivatives as substrate-based inhibitors of bacterial efflux pumps. Eur $J$ Med Chem 2008, 43 (11), 2453-63.

[105] Pieroni, M.; Dimovska, M.; Brincat, J. P.; Sabatini, S.; Carosati, E.; Massari, S.; Kaatz, G. W.; Fravolini, A., From 6-aminoquinolone antibacterials to 6-amino-7thiopyranopyridinylquinolone ethyl esters as inhibitors of Staphylococcus aureus multidrug efflux pumps. J Med Chem 2010, 53 (11), 4466-80.

[106] Sabatini, S.; Gosetto, F.; Manfroni, G.; Tabarrini, O.; Kaatz, G. W.; Patel, D.; Cecchetti, V., Evolution from a natural flavones nucleus to obtain 2-(4Propoxyphenyl)quinoline derivatives as potent inhibitors of the $S$. aureus NorA efflux pump. J Med Chem 2011, 54 (16), 5722-36.

[107] Sabatini, S.; Gosetto, F.; Iraci, N.; Barreca, M. L.; Massari, S.; Sancineto, L.; Manfroni, G.; Tabarrini, O.; Dimovska, M.; Kaatz, G. W.; Cecchetti, V., Re-evolution of the 2-phenylquinolines: ligand-based design, synthesis, and biological evaluation of a potent new class of Staphylococcus aureus NorA efflux pump inhibitors to combat antimicrobial resistance. J Med Chem 2013, 56 (12), 4975-89.

[108] Thota, N.; Reddy, M. V.; Kumar, A.; Khan, I. A.; Sangwan, P. L.; Kalia, N. P.; Koul, J. L.; Koul, S., Substituted dihydronaphthalenes as efflux pump inhibitors of Staphylococcus aureus. Eur J Med Chem 2010, 45 (9), 360716.

[109] Fontaine, F.; Hequet, A.; Voisin-Chiret, A. S.; Bouillon, A.; Lesnard, A.; Cresteil, T.; Jolivalt, C.; Rault, S., First identification of boronic species as novel potential inhibitors of the Staphylococcus aureus NorA efflux pump. $J$ Med Chem 2014, 57 (6), 2536-48.

[110] Fontaine, F.; Hequet, A.; Voisin-Chiret, A. S.; Bouillon, A.; Lesnard, A.; Cresteil, T.; Jolivalt, C.; Rault, S., Boronic species as promising inhibitors of the Staphylococcus aureus NorA efflux pump: study of 6substituted pyridine-3-boronic acid derivatives. Eur J Med Chem 2015, 95, 185-98.

[111] Brincat, J. P.; Carosati, E.; Sabatini, S.; Manfroni, G.; Fravolini, A.; Raygada, J. L.; Patel, D.; Kaatz, G. W.; Cruciani, G., Discovery of novel inhibitors of the NorA multidrug transporter of Staphylococcus aureus. J Med Chem 2011, 54 (1), 354-65. 
[112] Vidaillac, C.; Guillon, J.; Arpin, C.; Forfar-Bares, I.; Ba, B. B.; Grellet, J.; Moreau, S.; Caignard, D. H.; Jarry, C.; Quentin, C., Synthesis of omeprazole analogues and evaluation of these as potential inhibitors of the multidrug efflux pump NorA of Staphylococcus aureus. Antimicrob Agents Chemother 2007, 51 (3), 831-8.

[113] Dalton, W. S.; Grogan, T. M.; Meltzer, P. S.; Scheper, R. J.; Durie, B. G.; Taylor, C. W.; Miller, T. P.; Salmon, S. E., Drug-resistance in multiple myeloma and non-Hodgkin's lymphoma: detection of P-glycoprotein and potential circumvention by addition of verapamil to chemotherapy. $J$ Clin Oncol 1989, 7 (4), 415-24.

[114] Aeschlimann, J. R.; Dresser, L. D.; Kaatz, G. W.; Rybak, M. J., Effects of NorA inhibitors on in vitro antibacterial activities and postantibiotic effects of levofloxacin, ciprofloxacin, and norfloxacin in genetically related strains of Staphylococcus aureus. Antimicrob Agents Chemother 1999, 43 (2), 335-40.

[115] Ng, E. Y.; Trucksis, M.; Hooper, D. C., Quinolone resistance mediated by nor $A$ : physiologic characterization and relationship to $f l q B$, a quinolone resistance locus on the Staphylococcus aureus chromosome. Antimicrob Agents Chemother 1994, 38 (6), 1345-55.

[116] Vidaillac, C.; Guillon, J.; Moreau, S.; Arpin, C.; Lagardere, A.; Larrouture, S.; Dallemagne, P.; Caignard, D. H.; Quentin, C.; Jarry, C., Synthesis of new 4-[2(alkylamino) ethylthio]pyrrolo[1,2-a]quinoxaline and 5-[2(alkylamino) ethylthio]pyrrolo[1,2-a]thieno[3,2-e]pyrazine derivatives, as potential bacterial multidrug resistance pump inhibitors. J Enzyme Inhib Med Chem 2007, 22 (5), 620-31. [117] Mullin, S.; Mani, N.; Grossman, T. H., Inhibition of Antibiotic Efflux in Bacteria by the Novel Multidrug Resistance Inhibitors Biricodar (VX-710) and Timcodar (VX-853). Antimicrob Agents Chemother 2004, 48 (11), 4171-4176.

[118] Bharate, J. B.; Singh, S.; Wani, A.; Sharma, S.; Joshi, P.; Khan, I. A.; Kumar, A.; Vishwakarma, R. A.; Bharate, S. B., Discovery of 4-acetyl-3-(4-fluorophenyl)-1-( $p$-tolyl)-5methylpyrrole as a dual inhibitor of human P-glycoprotein and Staphylococcus aureus NorA efflux pump. Org Biomol Chem 2015, 13 (19), 5424-31.

[119] Leitner, I.; Nemeth, J.; Feurstein, T.; Abrahim, A.; Matzneller, P.; Lagler, H.; Erker, T.; Langer, O.; Zeitlinger, M., The third-generation P-glycoprotein inhibitor tariquidar may overcome bacterial multidrug resistance by increasing intracellular drug concentration. J Antimicrob Chemother 2011, 66 (4), 834-9.

[120] Kaatz, G. W.; Moudgal, V. V.; Seo, S. M.; Hansen, J. B.; Kristiansen, J. E., Phenylpiperidine selective serotonin reuptake inhibitors interfere with multidrug efflux pump activity in Staphylococcus aureus. Int J Antimicrob Agents 2003, 22 (3), 254-61.

[121] Wei, P.; Kaatz, G. W.; Kerns, R. J., Structural differences between paroxetine and femoxetine responsible for differential inhibition of Staphylococcus aureus efflux pumps. Bioorg Med Chem Lett 2004, 14 (12), 3093-7.

[122] German, N.; Kaatz, G. W.; Kerns, R. J., Synthesis and evaluation of PSSRI-based inhibitors of Staphylococcus aureus multidrug efflux pumps. Bioorg Med Chem Lett 2008, 18 (4), 1368-73.

[123] Kalle, A. M.; Rizvi, A., Inhibition of Bacterial Multidrug Resistance by Celecoxib, a Cyclooxygenase-2 Inhibitor. Antimicrob Agents Chemother 2011, 55 (1), 439442.

[124] Sabatini, S.; Gosetto, F.; Serritella, S.; Manfroni, G.; Tabarrini, O.; Iraci, N.; Brincat, J. P.; Carosati, E.; Villarini, M.; Kaatz, G. W.; Cecchetti, V., Pyrazolo[4,3c] [1,2]benzothiazines 5,5-dioxide: a promising new class of Staphylococcus aureus NorA efflux pump inhibitors. $J$ Med Chem 2012, 55 (7), 3568-72.

[125] Kaatz, G. W.; Moudgal, V. V.; Seo, S. M.; Kristiansen, J. E., Phenothiazines and thioxanthenes inhibit multidrug efflux pump activity in Staphylococcus aureus. Antimicrob Agents Chemother 2003, 47 (2), 719-26.

[126] Sabatini, S.; Kaatz, G. W.; Rossolini, G. M.; Brandini, D.; Fravolini, A., From phenothiazine to 3-phenyl-1,4benzothiazine derivatives as inhibitors of the Staphylococcus aureus NorA multidrug efflux pump. J Med Chem 2008, 51 (14), 4321-30.

[127] Kristiansen, M. M.; Leandro, C.; Ordway, D.; Martins, M.; Viveiros, M.; Pacheco, T.; Molnar, J.; Kristiansen, J. E.; Amaral, L., Thioridazine reduces resistance of methicillinresistant Staphylococcus aureus by inhibiting a reserpinesensitive efflux pump. In Vivo 2006, 20 (3), 361-6.

[128] Kristiansen, J. E.; Hendricks, O.; Delvin, T.; Butterworth, T. S.; Aagaard, L.; Christensen, J. B.; Flores, V. C.; Keyzer, H., Reversal of resistance in microorganisms by help of non-antibiotics. J Antimicrob Chemother 2007, 59 (6), 1271-9.

[129] Alnaseri, H.; Arsic, B.; Schneider, J. E.; Kaiser, J. C.; Scinocca, Z. C.; Heinrichs, D. E.; McGavin, M. J., Inducible Expression of a Resistance-Nodulation-Division-Type Efflux Pump in Staphylococcus aureus Provides Resistance to Linoleic and Arachidonic Acids. J Bacteriol 2015, 197 (11), 1893-905.

[130] Schindler, B. D.; Frempong-Manso, E.; DeMarco, C. E.; Kosmidis, C.; Matta, V.; Seo, S. M.; Kaatz, G. W., Analyses of multidrug efflux pump-like proteins encoded on the Staphylococcus aureus chromosome. Antimicrob Agents Chemother 2015, 59 (1), 747-8.

[131] Murakami, S.; Nakashima, R.; Yamashita, E.; Yamaguchi, A., Crystal structure of bacterial multidrug efflux transporter AcrB. Nature 2002, 419 (6907), 587-93.

[132] Kim, J.-S.; Jeong, H.; Song, S.; Kim, H.-Y.; Lee, K.; Hyun, J.; Ha, N.-C., Structure of the Tripartite Multidrug Efflux Pump AcrAB-TolC Suggests an Alternative Assembly Mode. Mol Cells 2015, 38 (2), 180-186.

[133] Du, D.; Wang, Z.; James, N. R.; Voss, J. E.; Klimont, E.; Ohene-Agyei, T.; Venter, H.; Chiu, W.; Luisi, B. F., Structure of the AcrAB-TolC multidrug efflux pump. Nature 2014, 509 (7501), 512-515.

[134] Pan, Y. P.; Xu, Y. H.; Wang, Z. X.; Fang, Y. P.; Shen, J. L., Overexpression of MexAB-OprM efflux pump in carbapenem-resistant Pseudomonas aeruginosa. Arch Microbiol 2016, 198 (6), 565-71. 
[135] Lorenzi, V.; Muselli, A.; Bernardini, A. F.; Berti, L.; Pages, J. M.; Amaral, L.; Bolla, J. M., Geraniol restores antibiotic activities against multidrug-resistant isolates from Gram-negative species. Antimicrob Agents Chemother 2009, 53 (5), 2209-11.

[136] Aparna, V.; Dineshkumar, K.; Mohanalakshmi, N.; Velmurugan, D.; Hopper, W., Identification of Natural Compound Inhibitors for Multidrug Efflux Pumps of Escherichia coli and Pseudomonas aeruginosa Using In Silico High-Throughput Virtual Screening and In Vitro Validation. PLOS ONE 2014, 9 (7), e101840.

[137] Ohene-Agyei, T.; Mowla, R.; Rahman, T.; Venter, H., Phytochemicals increase the antibacterial activity of antibiotics by acting on a drug efflux pump. Microbiologyopen 2014, 3 (6), 885-96.

[138] Negi, N.; Prakash, P.; Gupta, M. L.; Mohapatra, T. M., Possible Role of Curcumin as an Efflux Pump Inhibitor in Multi Drug Resistant Clinical Isolates of Pseudomonas aeruginosa. J Clin Diagn Res 2014, 8 (10), DC04-7.

[139] Lee, M. D.; Galazzo, J. L.; Staley, A. L.; Lee, J. C.; Warren, M. S.; Fuernkranz, H.; Chamberland, S.; Lomovskaya, O.; Miller, G. H., Microbial fermentationderived inhibitors of efflux-pump-mediated drug resistance. Farmaco 2001, 56 (1-2), 81-5.

[140] Nguyen, S. T.; Kwasny, S. M.; Ding, X.; Cardinale, S. C.; McCarthy, C. T.; Kim, H. S.; Nikaido, H.; Peet, N. P.; Williams, J. D.; Bowlin, T. L.; Opperman, T. J., Structureactivity relationships of a novel pyranopyridine series of Gram-negative bacterial efflux pump inhibitors. Bioorg Med Chem 2015, 23 (9), 2024-34.

[141] Opperman, T. J.; Kwasny, S. M.; Kim, H. S.; Nguyen, S. T.; Houseweart, C.; D'Souza, S.; Walker, G. C.; Peet, N. P.; Nikaido, H.; Bowlin, T. L., Characterization of a novel pyranopyridine inhibitor of the AcrAB efflux pump of Escherichia coli. Antimicrob Agents Chemother 2014, 58 (2), 722-33.

[142] Renau, T. E.; Leger, R.; Flamme, E. M.; Sangalang, J.; She, M. W.; Yen, R.; Gannon, C. L.; Griffith, D.; Chamberland, S.; Lomovskaya, O.; Hecker, S. J.; Lee, V. J.; Ohta, T.; Nakayama, K., Inhibitors of efflux pumps in Pseudomonas aeruginosa potentiate the activity of the fluoroquinolone antibacterial levofloxacin. $J$ Med Chem 1999, 42 (24), 4928-31.

[143] Lomovskaya, O.; Warren, M. S.; Lee, A.; Galazzo, J.; Fronko, R.; Lee, M.; Blais, J.; Cho, D.; Chamberland, S.; Renau, T.; Leger, R.; Hecker, S.; Watkins, W.; Hoshino, K.; Ishida, H.; Lee, V. J., Identification and characterization of inhibitors of multidrug resistance efflux pumps in Pseudomonas aeruginosa: novel agents for combination therapy. Antimicrob Agents Chemother 2001, 45 (1), 105-16. [144] Renau, T. E.; Leger, R.; Flamme, E. M.; She, M. W.; Gannon, C. L.; Mathias, K. M.; Lomovskaya, O.; Chamberland, S.; Lee, V. J.; Ohta, T.; Nakayama, K.; Ishida, Y., Addressing the stability of C-capped dipeptide efflux pump inhibitors that potentiate the activity of levofloxacin in Pseudomonas aeruginosa. Bioorg Med Chem Lett 2001, 11 (5), 663-7.
[145] Renau, T. E.; Leger, R.; Filonova, L.; Flamme, E. M.; Wang, M.; Yen, R.; Madsen, D.; Griffith, D.; Chamberland, S.; Dudley, M. N.; Lee, V. J.; Lomovskaya, O.; Watkins, W. J.; Ohta, T.; Nakayama, K.; Ishida, Y., Conformationallyrestricted analogues of efflux pump inhibitors that potentiate the activity of levofloxacin in Pseudomonas aeruginosa. Bioorg Med Chem Lett 2003, 13 (16), 2755-8.

[146] Renau, T. E.; Leger, R.; Yen, R.; She, M. W.; Flamme, E. M.; Sangalang, J.; Gannon, C. L.; Chamberland, S.; Lomovskaya, O.; Lee, V. J., Peptidomimetics of efflux pump inhibitors potentiate the activity of levofloxacin in Pseudomonas aeruginosa. Bioorg Med Chem Lett 2002, 12 (5), 763-6.

[147] Vargiu, A. V.; Ruggerone, P.; Opperman, T. J.; Nguyen, S. T.; Nikaido, H., Molecular mechanism of MBX2319 inhibition of Escherichia coli AcrB multidrug efflux pump and comparison with other inhibitors. Antimicrob Agents Chemother 2014, 58 (10), 6224-34.

[148] Sjuts, H.; Vargiu, A. V.; Kwasny, S. M.; Nguyen, S. T.; Kim, H. S.; Ding, X.; Ornik, A. R.; Ruggerone, P.; Bowlin, T. L.; Nikaido, H.; Pos, K. M.; Opperman, T. J., Molecular basis for inhibition of AcrB multidrug efflux pump by novel and powerful pyranopyridine derivatives. Proc Natl Acad Sci U S A 2016, 113 (13), 3509-14.

[149] Bohnert, J. A.; Kern, W. V., Selected arylpiperazines are capable of reversing multidrug resistance in Escherichia coli overexpressing RND efflux pumps. Antimicrob Agents Chemother 2005, 49 (2), 849-52.

[150] Kern, W. V.; Steinke, P.; Schumacher, A.; Schuster, S.; von Baum, H.; Bohnert, J. A., Effect of 1-(1naphthylmethyl)-piperazine, a novel putative efflux pump inhibitor, on antimicrobial drug susceptibility in clinical isolates of Escherichia coli. J Antimicrob Chemother 2006, 57 (2), 339-43.

[151] Opperman, T. J.; Nguyen, S. T., Recent advances toward a molecular mechanism of efflux pump inhibition. Front Microbiol 2015, 6, 421.

[152] Nakayama, K.; Ishida, Y.; Ohtsuka, M.; Kawato, H.; Yoshida, K.-i.; Yokomizo, Y.; Hosono, S.; Ohta, T.; Hoshino, K.; Ishida, H.; Yoshida, K.; Renau, T. E.; Léger, R.; Zhang, J. Z.; Lee, V. J.; Watkins, W. J., MexAB-OprMSpecific efflux pump inhibitors in Pseudomonas aeruginosa. Part 1: Discovery and early strategies for lead optimization. Bioorg Med Chem Lett 2003, 13 (23), 4201-4204.

[153] Nakayama, K.; Ishida, Y.; Ohtsuka, M.; Kawato, H.; Yoshida, K.; Yokomizo, Y.; Ohta, T.; Hoshino, K.; Otani, T.; Kurosaka, Y.; Yoshida, K.; Ishida, H.; Lee, V. J.; Renau, T. E.; Watkins, W. J., MexAB-OprM specific efflux pump inhibitors in Pseudomonas aeruginosa. Part 2: achieving activity in vivo through the use of alternative scaffolds. Bioorg Med Chem Lett 2003, 13 (23), 4205-8.

[154] Nakayama, K.; Kawato, H.; Watanabe, J.; Ohtsuka, M.; Yoshida, K.; Yokomizo, Y.; Sakamoto, A.; Kuru, N.; Ohta, T.; Hoshino, K.; Yoshida, K.; Ishida, H.; Cho, A.; Palme, M. H.; Zhang, J. Z.; Lee, V. J.; Watkins, W. J., MexAB-OprM specific efflux pump inhibitors in Pseudomonas aeruginosa. Part 3: Optimization of potency in the pyridopyrimidine series through the application of a 
pharmacophore model. Bioorg Med Chem Lett 2004, 14 (2), 475-9.

[155] Nakayama, K.; Kuru, N.; Ohtsuka, M.; Yokomizo, Y.; Sakamoto, A.; Kawato, H.; Yoshida, K.; Ohta, T.; Hoshino, K.; Akimoto, K.; Itoh, J.; Ishida, H.; Cho, A.; Palme, M. H.; Zhang, J. Z.; Lee, V. J.; Watkins, W. J., MexAB-OprM specific efflux pump inhibitors in Pseudomonas aeruginosa. Part 4: Addressing the problem of poor stability due to photoisomerization of an acrylic acid moiety. Bioorg Med Chem Lett 2004, 14 (10), 2493-7.

[156] Yoshida, K.; Nakayama, K.; Kuru, N.; Kobayashi, S.; Ohtsuka, M.; Takemura, M.; Hoshino, K.; Kanda, H.; Zhang, J. Z.; Lee, V. J.; Watkins, W. J., MexAB-OprM specific efflux pump inhibitors in Pseudomonas aeruginosa. Part 5: Carbon-substituted analogues at the C-2 position. Bioorg Med Chem 2006, 14 (6), 1993-2004.

[157] Yoshida, K.; Nakayama, K.; Yokomizo, Y.; Ohtsuka, M.; Takemura, M.; Hoshino, K.; Kanda, H.; Namba, K.; Nitanai, H.; Zhang, J. Z.; Lee, V. J.; Watkins, W. J., MexAB-OprM specific efflux pump inhibitors in Pseudomonas aeruginosa. Part 6: exploration of aromatic substituents. Bioorg Med Chem 2006, 14 (24), 8506-18. [158] Yoshida, K.; Nakayama, K.; Ohtsuka, M.; Kuru, N.; Yokomizo, Y.; Sakamoto, A.; Takemura, M.; Hoshino, K.; Kanda, H.; Nitanai, H.; Namba, K.; Yoshida, K.; Imamura, Y.; Zhang, J. Z.; Lee, V. J.; Watkins, W. J., MexAB-OprM specific efflux pump inhibitors in Pseudomonas aeruginosa. Part 7: highly soluble and in vivo active quaternary ammonium analogue D13-9001, a potential preclinical candidate. Bioorg Med Chem 2007, 15 (22), 7087-97.

[159] Mallea, M.; Mahamoud, A.; Chevalier, J.; AlibertFranco, S.; Brouant, P.; Barbe, J.; Pages, J. M., Alkylaminoquinolines inhibit the bacterial antibiotic efflux pump in multidrug-resistant clinical isolates. Biochem $\mathrm{J}$ 2003, 376 (Pt 3), 801-5.

[160] Chevalier, J.; Bredin, J.; Mahamoud, A.; Mallea, M.; Barbe, J.; Pages, J. M., Inhibitors of antibiotic efflux in resistant Enterobacter aerogenes and Klebsiella pneumoniae strains. Antimicrob Agents Chemother 2004, 48 (3), 1043-6. [161] Ghisalberti, D.; Mahamoud, A.; Chevalier, J.; Baitiche, M.; Martino, M.; Pages, J. M.; Barbe, J., Chloroquinolines block antibiotic efflux pumps in antibiotic-resistant Enterobacter aerogenes isolates. Int $J$ Antimicrob Agents 2006, 27 (6), 565-9.

[162] Mahamoud, A.; Chevalier, J.; Baitiche, M.; Adam, E.; Pages, J. M., An alkylaminoquinazoline restores antibiotic activity in Gram-negative resistant isolates. Microbiology 2011, 157 (Pt 2), 566-71.

[163] Yilmaz, S.; Altinkanat-Gelmez, G.; Bolelli, K.; Guneser-Merdan, D.; Over-Hasdemir, M. U.; Yildiz, I.; AkiYalcin, E.; Yalcin, I., Pharmacophore generation of 2substituted benzothiazoles as AdeABC efflux pump inhibitors in A. baumannii. SAR QSAR Environ Res 2014, 25 (7), 551-63.

[164] Yilmaz, S.; Altinkanat-Gelmez, G.; Bolelli, K.; Guneser-Merdan, D.; Ufuk Over-Hasdemir, M.; Aki-Yalcin, E.; Yalcin, I., Binding site feature description of 2substituted benzothiazoles as potential AcrAB-TolC efflux pump inhibitors in E. coli. SAR QSAR Environ Res 2015, 26 (10), 853-71.

[165] Zeng, B.; Wang, H.; Zou, L.; Zhang, A.; Yang, X.; Guan, Z., Evaluation and target validation of indole derivatives as inhibitors of the AcrAB-TolC efflux pump. Biosci Biotechnol Biochem 2010, 74 (11), 2237-41.

[166] Bohnert, J. A.; Szymaniak-Vits, M.; Schuster, S.; Kern, W. V., Efflux inhibition by selective serotonin reuptake inhibitors in Escherichia coli. $J$ Antimicrob Chemother 2011, 66 (9), 2057-60.

[167] Piddock, L. J.; Garvey, M. I.; Rahman, M. M.; Gibbons, S., Natural and synthetic compounds such as trimethoprim behave as inhibitors of efflux in Gram-negative bacteria. J Antimicrob Chemother 2010, 65 (6), 1215-23.

[168] Li, B.; Yao, Q.; Pan, X. C.; Wang, N.; Zhang, R.; Li, J.; Ding, G.; Liu, X.; Wu, C.; Ran, D.; Zheng, J.; Zhou, H., Artesunate enhances the antibacterial effect of $\beta$-lactam antibiotics against Escherichia coli by increasing antibiotic accumulation via inhibition of the multidrug efflux pump system AcrAB-TolC. J Antimicrob Chemother 2011, 66 (4), 769-77.

[169] Bay, D. C.; Rommens, K. L.; Turner, R. J., Small multidrug resistance proteins: A multidrug transporter family that continues to grow. Biochim Biophys Acta 2008, 1778 (9), 1814-1838.

[170] Chen, Y.-J.; Pornillos, O.; Lieu, S.; Ma, C.; Chen, A. P.; Chang, G., X-ray structure of EmrE supports dual topology model. Proc Natl Acad Sci 2007, 104 (48), 1899919004.

[171] Suriyanarayanan, B.; Sarojini Santhosh, R., Docking analysis insights quercetin can be a non-antibiotic adjuvant by inhibiting Mmr drug efflux pump in Mycobacterium sp. and its homologue EmrE in Escherichia coli. J Biomol Struct Dyn 2015, 33 (8), 1819-34.

[172] Malkhed, V.; Mustyala, K. K.; Potlapally, S. R.; Vuruputuri, U., Identification of novel leads applying in silico studies for Mycobacterium multidrug resistant (MMR) protein. J Biomol Struct Dyn 2014, 32 (12), 1889-906.

[173] van Veen, H. W., Structural biology: Last of the multidrug transporters. Nature 2010, 467 (7318), 926-927.

[174] Tsuda, M.; Terada, T.; Asaka, J.; Ueba, M.; Katsura, $\mathrm{T}$.; Inui, K., Oppositely directed $\mathrm{H}+$ gradient functions as a driving force of rat $\mathrm{H}+$ /organic cation antiporter MATE1. Am J Physiol Renal Physiol 2007, 292 (2), F593-8.

[175] Terada, T.; Inui, K., Physiological and pharmacokinetic roles of $\mathrm{H}+/$ organic cation antiporters (MATE/SLC47A). Biochem Pharmacol 2008, 75 (9), 168996.

[176] Tanaka, Y.; Hipolito, C. J.; Maturana, A. D.; Ito, K.; Kuroda, T.; Higuchi, T.; Katoh, T.; Kato, H. E.; Hattori, M.; Kumazaki, K.; Tsukazaki, T.; Ishitani, R.; Suga, H.; Nureki, O., Structural basis for the drug extrusion mechanism by a MATE multidrug transporter. Nature 2013, 496 (7444), 24751.

[177] Radchenko, M.; Symersky, J.; Nie, R.; Lu, M., Structural basis for the blockade of MATE multidrug efflux pumps. Nat Commun 2015, 6, 7995. 
[178] Omote, H.; Hiasa, M.; Matsumoto, T.; Otsuka, M.; Moriyama, Y., The MATE proteins as fundamental transporters of metabolic and xenobiotic organic cations. Trends Pharmacol Sci 2006, 27 (11), 587-93.

[179] Moriyama, Y.; Hiasa, M.; Matsumoto, T.; Omote, H., Multidrug and toxic compound extrusion (MATE)-type proteins as anchor transporters for the excretion of metabolic waste products and xenobiotics. Xenobiotica 2008, 38 (7-8), 1107-18.

[180] Kuroda, T.; Tsuchiya, T., Multidrug efflux transporters in the MATE family. Biochim Biophys Acta 2009, 1794 (5), 763-8.

[181] Du, D.; van Veen, H. W.; Murakami, S.; Pos, K. M.; Luisi, B. F., Structure, mechanism and cooperation of bacterial multidrug transporters. Curr Opin Struct Biol 2015, 33, 76-91.

[182] Kesherwani, M.; Michael Gromiha, M.; Fukui, K.; Velmurugan, D., Identification of novel natural inhibitor for NorM - a multidrug and toxic compound extrusion transporter - an in silico molecular modeling and simulation studies. J Biomol Struct Dyn 2017, 35 (1), 58-77. 


\section{Title page}

\section{Medicinal Chemistry Updates on Bacterial Efflux Pump Modulators}

F. Durães - Fernando André Pereira Marques Durães

Laboratory of Organic and Pharmaceutical Chemistry, Department of Chemical Sciences, Faculty of Pharmacy, University of Porto, Rua Jorge Viterbo Ferreira, 228, 4050-313 Porto, Portugal.

CIIMAR/CIMAR - Interdisciplinary Centre of Marine and Environmental Research, University of Porto. Terminal de Cruzeiros do Porto de Leixões, Avenida General Norton de Matos P 4450-208 Matosinhos, Portugal.

M. M. Pinto - Madalena Maria Magalhães Pinto

Laboratory of Organic and Pharmaceutical Chemistry, Department of Chemical Sciences, Faculty of Pharmacy, University of Porto, Rua Jorge Viterbo Ferreira, 228, 4050-313 Porto, Portugal.

CIIMAR/CIMAR - Interdisciplinary Centre of Marine and Environmental Research, University of Porto. Terminal de Cruzeiros do Porto de Leixões, Avenida General Norton de Matos P 4450-208 Matosinhos, Portugal.

E. Sousa (corresponding author) - Maria Emília Silva Pereira de Sousa

Laboratory of Organic and Pharmaceutical Chemistry, Department of Chemical Sciences, Faculty of Pharmacy, University of Porto, Rua Jorge Viterbo Ferreira, 228, 4050-313 Porto, Portugal. tel: +351220428689, fax: +351226093390, esousa@ff.up.pt

CIIMAR/CIMAR - Interdisciplinary Centre of Marine and Environmental Research, University of Porto. Terminal de Cruzeiros do Porto de Leixões, Avenida General Norton de Matos P 4450-208 Matosinhos, Portugal.

XXX-XXX/14 \$58.00+.00 (c) 2014 Bentham Science Publishers 


\title{
Medicinal Chemistry Updates on Bacterial Efflux Pump Modulators
}

\author{
Fernando Durães ${ }^{\mathrm{a}}$, Madalena Pinto ${ }^{\mathrm{a}}$, Emília Sousa ${ }^{* a}$
}

a Laboratory of Organic and Pharmaceutical Chemistry, Department of Chemical Sciences, Faculty of Pharmacy, University of Porto, Rua Jorge Viterbo Ferreira, 228, 4050-313 Porto, Portugal and CIIMAR/CIMAR - Interdisciplinary Centre of Marine and Environmental Research, University of Porto. Terminal de Cruzeiros do Porto de Leixões, Avenida General Norton de Matos P 4450-208 Matosinhos, Portugal. tel: +351220428689, fax: +351226093390, esousa@ff.up.pt.

\begin{abstract}
Antibiotic resistance is one of the most pressing health issues of our days. It can arise due to a multiplicity of factors, such as target modification, decrease in the drug uptake, changes in the metabolic pathways and activation of efflux pumps. The overexpression of efflux pumps is responsible for the extrusion of drugs, making antibiotic therapy fail, as the quantity of intracellular antibiotic is not enough to provide the desired therapeutic effect.

Efflux pumps can be included in five families according to their composition, nature of substrates, energy source, and number of transmembrane spanning regions. The ABC superfamily are mainly found in Gram-positive bacteria, use ATP as an energy source, and only a limited number of $\mathrm{ABC}$ pumps confer multidrug resistance (MDR).

On the other hand, the MFS family, most present in Gram-positive bacteria, and the RND family, characteristic of Gramnegative bacteria, are the most associated with antibiotic resistance. A wide variety of inhibitors have been disclosed for both families, from either natural or synthetic sources, or even drugs that are currently in therapy for other diseases.

The other two families are the SMR, which are the smallest drug efflux proteins known, and the MATE family, whose pumps can also resort to the sodium gradient as an energy source.

In this review, it is intended to present a comprehensive review of the classes of efflux pump inhibitors from the various sources, highlighting their structure-activity relationships, which can be useful for medicinal chemists in the pursuit of novel efflux pump inhibitors.
\end{abstract}

Keywords: Antimicrobial resistance; efflux pump inhibitors; natural products; synthetic compounds; existing drugs; Structure-activity relationship.

\section{INTRODUCTION}

Bacteria were discovered the late $19^{\text {th }}$ century as infectious agents. It was, however, half a century later that antibiotics completely revolutionized medicine and became a turning point in human history. Unfortunately, the misruled use of these drugs led to the appearance of resistant strains, and to the concern of a return to the pre-antibiotic era, where the simplest, most treatable infections can become deadly [1-2]. Antibiotic resistance can arise due to multiple causes. The most common are the overuse and/or inappropriate prescribing, as well as extensive veterinary and agricultural use. These factors are especially alarming if it is taken into account the very few new antibiotics that were discovered in the past years [3]. Due to their genetic plasticity, bacteria can develop defense mechanisms in response to environmental threats. As far as antibiotics are concerned, bacteria use two major strategies to adapt to these drugs, which are mutations in genes and the acquisition of resistance genes through horizontal gene transfer. The first strategy can be manifested through modification of the target, decrease in the drug uptake, activation of efflux pumps to extrude the drug or changes in metabolic pathways. The latter is related to the acquisition of external genetic material through transformation, transduction, or conjugation [4-5]. Recently, vesicle-mediated resistance has also been regarded as a mechanism of resistance acquisition [6].

Efflux pumps are ubiquitous in bacteria, and can be encoded by genes present either in the bacterial chromosomes or in plasmids [7-8]. They concern five families, according to their composition, substrates, energy sources, and number of transmembrane spanning regions: the adenosine triphosphate (ATP)-binding cassette (ABC) superfamily, the resistancenodulation-division family (RND), the major facilitator superfamily (MFS), the small multidrug resistance (SMR) family, and the multidrug and toxic compound extrusion (MATE) family (Figure 1) [7-12].

\footnotetext{
*Address correspondence to this author at the Department of Organic and Pharmaceutical Chemistry, Faculty of Pharmacy, University of Porto, P.O. Box: 4050-313, Porto, Portugal; Tel/Fax: +351 220428 689, +351 226093 390; E-mail: esousa@ff.up.pt
} 


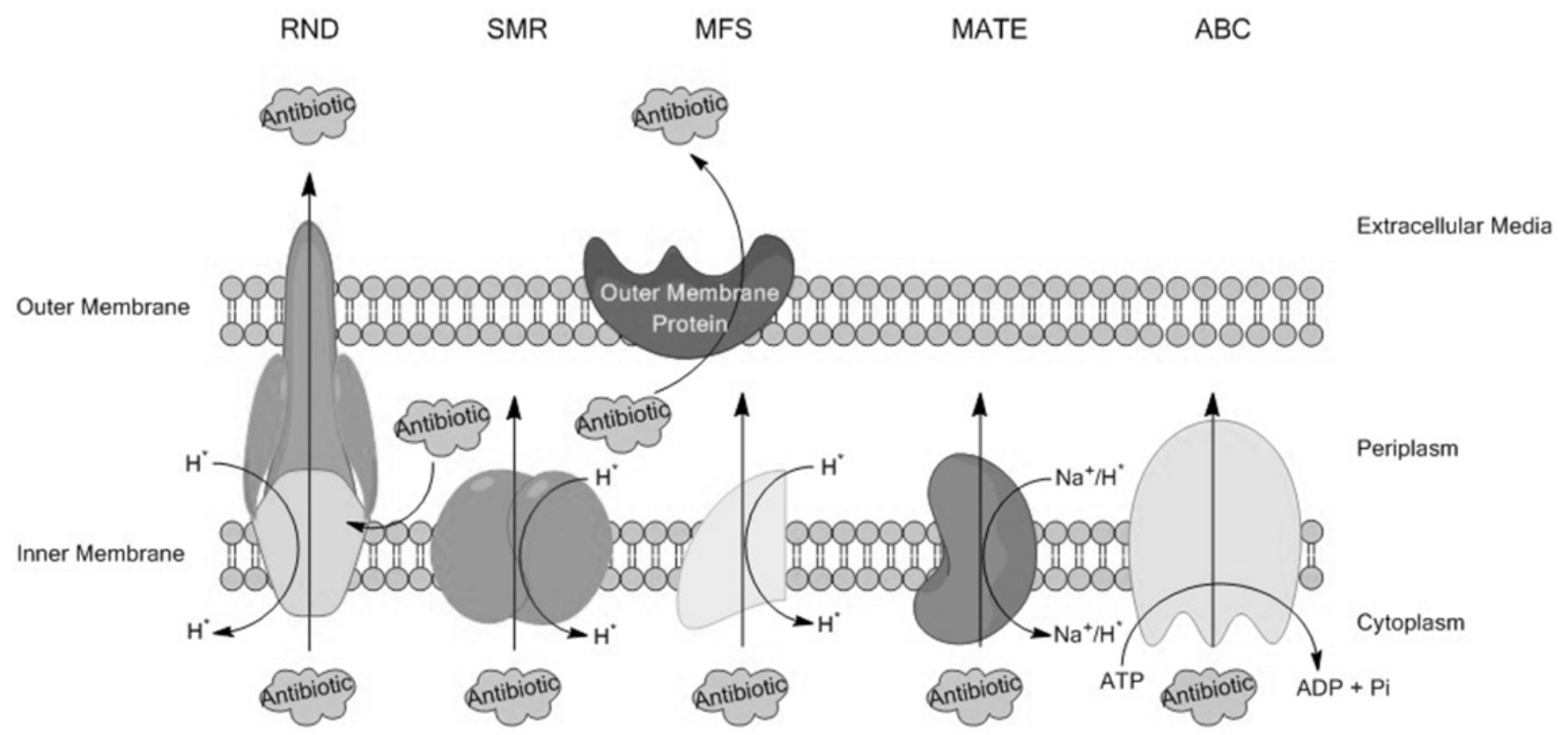

Figure 1 Representation of the five efflux pump families (adapted from [8]).

Efflux pumps can be further divided into two major families, the primary transporters, which hydrolyze ATP as a source of energy, as is the case of the $\mathrm{ABC}$ family, and secondary transporters, which use the proton or sodium gradient as a source of energy [13].

Overexpression of multidrug efflux pumps is correlated with an increase in the minimum inhibitory concentration (MIC) of antibiotics, and thus, these pumps can be considered potential antibacterial targets. Therefore, the development of efflux pump inhibitors may be a way of fighting against resistant microorganisms. Studies have been carried out on the structure of efflux pumps, as well as on their mechanisms of binding and extrusion, leading to the possibility of structure-based design of efflux pump inhibitors (EPIs). The rationale for the development of these EPIs could be, for instance, the inhibition of the energy sources required for the ability to extrude antibiotics, and the development of compounds that compete with the antibiotics for their extrusion. However, the main challenge lies in the fact that many compounds that present EPI activity and are already used for the treatment of other diseases are toxic for human cells in the concentrations required to present EPI activity and, therefore, clinically useless [7-9, 13].

Even though there are already several reviews which describe different classes of EPIs [11, 13-31], most focus on a particular source of compounds or on a class of pumps or bacteria. Herein, it is intended to present a comprehensive review of the several classes of EPIs from natural and synthetic sources and from existing drugs in therapeutics and to highlight structure-activity relationship studies in the referred efflux pumps (ABC, RND, MFS, SMR and MATE), which can be useful for medicinal chemists in the pursuit of novel compounds for this goal.

\section{2. $\mathbf{A B C}$}

The $\mathrm{ABC}$ transporters are primary transporters, and they exist in every membrane systems, whether the organism is prokaryotic or eukaryotic. P-Glycoprotein (P-gp) is a very well known human $\mathrm{ABC}$ transporter, involved in the resistance of cytotoxic drugs used in cancer therapy [32-34], whose modulation has been regarded as a study field [3537]. Structurally, an $\mathrm{ABC}$ transporter consists of four conserved domains, two transmembrane domains, and two cytoplasmic domains, which bind to nucleotides and, therefore, are involved in ATP binding [32, 38]. In fact, the nucleotide binding domains are responsible for hydrolyzing ATP, which will lead to conformational changes in the attached transmembrane domains, making it possible for substrates to cross the lipid bilayer of the membrane, either in an inwards or an outwards fashion [39].

The bacterial transporters are highly selective for sugars, amino acids, metallic cations, organo-iron complexes, vitamins, and antibiotics [32, 40]. They are found in Grampositive bacteria, such as Enterococcus faecalis and Staphylococcus aureus, and confer resistance to macrolides and bacitracin. However, only a limited number of $\mathrm{ABC}$ pumps are associated with MDR, which means that $A B C$ pumps do not play a pivotal role in conferring antibiotic resistance in bacteria [32-33].

As such, bacterial $\mathrm{ABC}$ transporter inhibitors are scarce. The few EPIs discovered in this context are mainly against MsrA (Figure 2, PDB 1NWA), whose tridimensional structure has been elucidated [41].

The structures of the $\mathrm{ABC}$ inhibitors that will be further detailed throughout this section are comprised in Figure 3. 


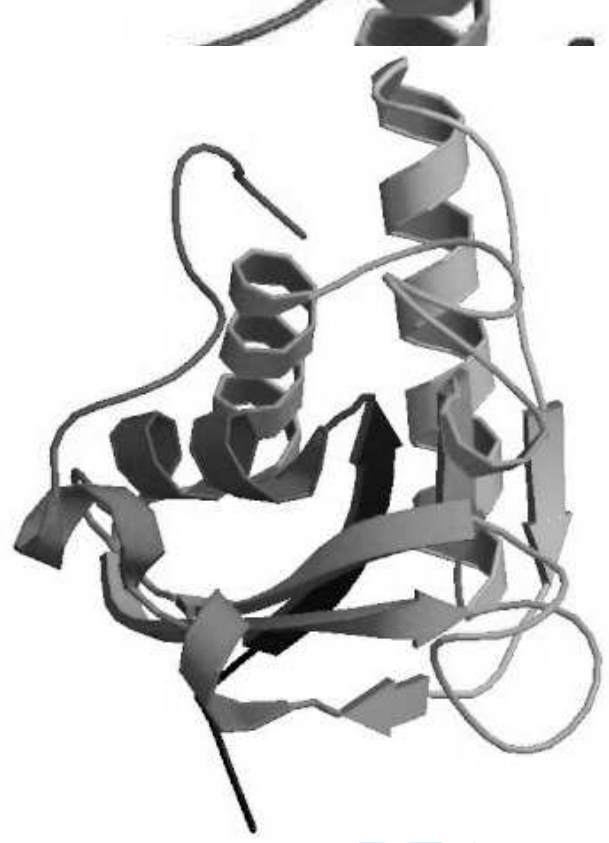

Figure 3 Crystal structure of MsrA, from Mycobacterium tuberculosis in complex with protein-bound methionine (PDB 1NWA).

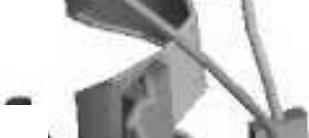

Principal Author Last Name et al.<smiles>[R]C/C=C(\C)CCCC(C)CCCC(C)CCCC(C)C</smiles><smiles>COc1ccc(-c2cc(=O)c3c(O)cc(O)cc3o2)cc1O</smiles>

11<smiles>[Z]C1CCC(=O)c2ccccc21</smiles>

12: $\mathrm{R}=-\mathrm{OH}$

13: $\mathrm{R}=$<smiles>COCc1cc(OC)c(OC)c(OC)c1</smiles><smiles>CCCCC/C=C\C/C=C\CCCCCCCC(=O)O</smiles>

Figure 2 Structures of $A B C$ inhibitors $1-20 . A c=A c e t y l$. 


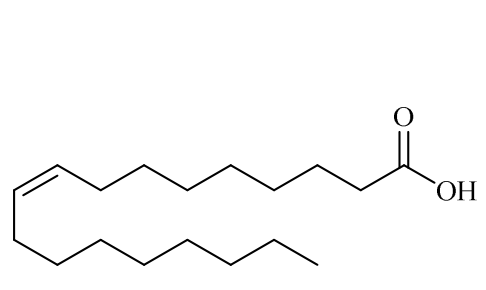

15

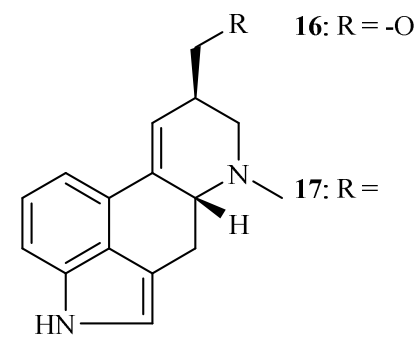<smiles>COC(=O)c1cc(OC)c(OC)c(OC)c1</smiles><smiles>[R]C(C)OC(=O)c1cccc([N+](=O)[O-])c1</smiles><smiles>COc1cc2c(cc1OC)CN(CCc1ccc(NC(=O)c3cccc4c(=O)c5cccc(OC)c5[nH]c34)cc1)CC2</smiles><smiles>O=[P+]([O-])([O-])[O-]</smiles>

19

20

Figure 3 (cont.)

acetoxy-5-hydroxygeranyllinalool (5), two diterpenoids [4344].

Phytol (6) is an acyclic isoprenoid, and its EPI activity against the ABC pumps of $E$. coli was assessed in combination with nalidixic acid. Additionally, 15 derivatives were synthesized. One derivative in particular, compound 7 , was able to decrease the MIC of nalidixic acid by 16 -fold, and four other derivatives reduced the MIC by 8-fold. This effect was caused by the inhibition of ATP-dependent efflux pump and down-regulation of the efflux gene. In silico absorption, distribution, metabolism, and excretion (ADME) analysis was performed, taking into account parameters such as solubility in water, human intestinal absorption, plasma protein binding, blood-brain barrier penetration, hepatotoxicity and cytochrome P450 (CYP450) inhibition. Results showed all five active derivatives presented low permeability into the central nervous system and displayed moderate intestinal absorption. In terms of plasma protein binding level, all five compounds exhibited levels below $90 \%$, suggesting favorable distribution of the compounds into the blood. They also did not present hepatotoxicity, unlike reserpine, making them good lead compounds [45].

Ursolic acid (8) is a pentacyclic triterpenoid isolated from diverse natural sources. Its wide activity includes antiinflammatory, antimicrobial, diuretic, and antitumor activity, among others. When tested against Escherichia coli strains that were resistant and susceptible to nalidixic acid, ursolic acid did not display intrinsic antibacterial activity. However, ursolic acid (8) was able to reduce the MIC of nalidixic acid by 2 -fold, when both drugs were used in combination. This synergy led to further research on this scaffold. The first derivative prepared was the 3-O-acetyl ursolic acid, and showed a 4-fold decrease in the MIC of nalidixic acid. The lipophilic nature of this derivative, allied with the increased potency, led to the synthesis of other lipophilic ester derivatives. The methyl and ethyl esters of 3-O-acetyl ursolic acid did not show any increment in the activity. However, a further increase in the ester carbon chain increased the drug reversal potential by 2 -fold, in comparison to 3 - $O$-acetyl ursolic acid, against the resistant strain. This is the case of the isopropyl (9) and the $n$-butyl (10) derivatives. The addition of a pentyl group led to a decrease in activity. Furthermore, ursolic acid (8), and derivatives 9 and 10, reduced the MIC of tetracycline by 2 -fold, 8 -fold, and 8 -fold, respectively. When these compounds were tested against ATP-dependent efflux pumps, it was found that derivatives 9 and 10 were successful in inhibiting these pumps in terms of the liberated inorganic phosphate. Molecular modeling studies with the YojI pump, using maltose/maltodextrin transport ATP-binding protein as a homology model, showed that 9 was bound to the nucleophilic Ser-78, to the aromatic Phe-81, Trp-85 and Leu-86, the acidic Glu-94 and Glu-144 and the basic Ala-79, Ala-139, Ala-140, Ala-141, Ala-143, Arg-74, Arg-122, Arg-146, Leu-107, Val-80, Lys-96, and Ile-123 in the nucleotide binding domain. Derivative 10 was also subjected to the same kind of studies using the same model, and was predicted to bind in the same region [46].

A study with flavones and isoflavones from the plant Sophora moorcroftiana revealed that diosmetin (11) was able to inhibit three drug resistant strains of $S$. aureus, including the ABC family MsrA overexpressing strain, potentiating the effects of norfloxacin and streptomycin [47]. These results corroborate the works of Chan et al (2013), who had previously studied the effects of diosmetin (11) as an EPI [48]. This group observed a synergistic effect of this flavonoid with erythromycin, which was 5-fold greater than that of verapamil [48].

4-Hydroxy- $\alpha$-tetralone (12) isolated from the plant Ammannia multiflora, showed enhancement against nalidixic acid resistant strains of Escherichia coli, prompting the synthesis of derivatives in order to infer their mechanism of action. Five derivatives were synthesized, and it was shown that compound 13 was able to inhibit the expression of the $\mathrm{ABC}$ transporter protein gene yojI, which encodes the $\mathrm{ABC}$ 
protein YojI, and was successful in combination with tetracycline [49]. Molecular modelling studies with compound 13 and a homology model of the YojI protein revealed the following bound residues: Ser-78 (nucleophilic), Leu-86, Ile-123, Ala-137, Ala-140, Ala-141, and Ala-143 (hydrophobic), Glu-144 (acidic), Arg-122 (basic), Phe-81 (aromatic), Glu-144 (acidic), Arg-146 and Lys-96 (basic), and Trp-85 (hydrophobic), the same region as noted for ursolic acid derivatives 9 and 10. Compound 13 showed good affinity and strong hydrophobic interaction with the YojI protein, corroborating the fact that this compound acts by the inhibition of ATP dependent efflux pumps [49].

The plant Portulaca oleraceae is used in folk medicine for its anti-inflammatory, anti-septic and diuretic effects, among others. A study of its fatty acids demonstrated that linoleic (14) and oleic acids (15) had synergistic activity with macrolides for the MsrA overexpressing strain of methicillin-resistant $S$. aureus (MRSA). In fact, erythromycin's activity was enhanced when in combination with either of these two compounds, likely through the inhibition of the MsrA ATP dependent pump. Since these fatty acids are essential for humans, low toxicity is expected, making them promising agents to use in combination with antibiotics [50].

Lysergol (16) is a clavine alkaloid of the ergoline family. Recently, its EPI activity was studied, and derivatives were synthesized [51]. The compounds were tested against susceptible and resistant to nalidixic acid strains of $E$. coli and a tetracycline resistant strain. While not possessing antibacterial activity, lysergol (16) showed synergy with nalidixic acid at the concentration of $10 \mu \mathrm{g} / \mathrm{ml}$, reducing its MIC by 8 -fold in the resistant strain of E. coli and 4-fold in the susceptible strain. Of the thirteen semi-synthetic derivatives prepared, derivatives $\mathbf{1 7}$ and $\mathbf{1 8}$ reduced nalidixic acid's MIC by 8 -fold, twice as much as lysergol (16), in the susceptible strain. A 4-fold potentiation of nalidixic acid was observed for the three compounds when tested against the resistant strain. As for the tetracycline resistant strain, all three compounds were able to reduce tetracycline's MIC by 8 -fold. Further studies were performed, in order to conclude whether or not these compounds were capable of interfering with ATP-dependent efflux pumps, and it was proven that all three compounds inhibited ATPase activity, concerning the liberation of inorganic phosphate. A down-regulation of the gene yojI was also observed when the tetracycline resistant strain was treated with lysergol (16) and its two most potent derivatives, $\mathbf{1 7}$ and 18, which further confirms the activity of these compounds as $\mathrm{ABC}$ transporter inhibitors [51].

\subsubsection{Existing Drugs}

As part of a screening program for P-gp inhibitors, GG918 (19) was discovered. This synthetic compound had already showed success in increasing the intracellular concentration of paclitaxel in cancer cells. In $S$. aureus, this compound was able to slightly potentiate the activity of norfloxacin in a strain overexpressing the ABC efflux pump
MsrA, suggesting that it may possess minimal activity over this pump [52].

The human P-gp inhibitors, vanadates, have been studied as inhibitors of the MutS pumps, belonging to the ABC ATPase superfamily. In fact, these compounds have demonstrated activity against the MutS in $P$. aeruginosa and $E$. coli. Decavanadate was the most effective, followed by orthovanadate (20), and they act non-competitively, possibly by replacing the inorganic phosphate. The fact that the $\mathrm{ABC}$ ATPases inhibited by orthovanadate are phosphorylated leads to the assumption that the low inhibitory effect could be attributed to the absence of a stable phosphorylation state of MutS [53].

\section{MFS}

The MFS is the most extensively studied family of transporters, present in both Gram-positive and Gramnegative bacteria, and comprises uniporters, symporters and antiporters. It also constitutes the largest family of secondary transporters, with over 10000 sequenced members. Its substrates are diverse, and can range from ions, to carbohydrates, lipids, amino acids, nucleosides, among others. As many members of the MFS originate bacterial MDR, these transporters can pose as a good system for modulation studies [7, 19, 38, 54].

With the elucidation of the crystal structure of the MFS pump EmrD of E. coli (Figure 4, PDB 2GFP) [55], a general structure could be proposed for MFS transporters: twelve transmembrane helices that form a compact structure, with four of these transmembrane helices facing away from the interior cavity and the rest forming the internal cavity, constituted mostly by hydrophobic residues [7].

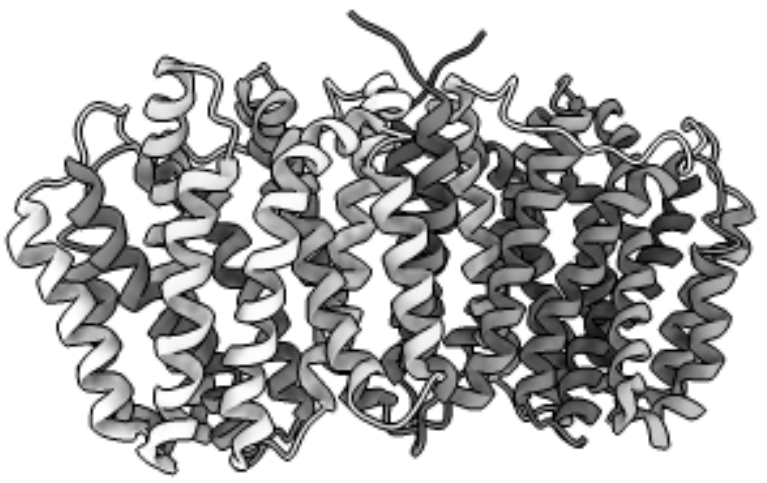

Figure 4 Crystal structure of EmrD, from E. coli (PDB 2GFP).

The efflux of drugs is only possible if the carrier alternates between two conformations, so that the substrate can bind to the convenient site in either side of the membrane. However, a limitation concerning the energy involved to displace the exposed hydrophilic surface of a protein can be inferred. It has been proved that either the oligomerization domain or the transport subunit provide the support needed to the movement of the substrate binding site, through a rigid-body 
rotation of the substrate binding domain, referred to as the "elevator mechanism" [56-57]. For this family, the most extensively studied efflux pump is the NorA pump of $S$. aureus.

The inhibitors that have proven activity against efflux pumps of the MFS family are gathered in Figure 5.<smiles>CC(C)=CCC/C(C)=C/CO</smiles>

21<smiles>C=CCc1ccc(OC)cc1</smiles>

22
23<smiles>CC(C)CCCC(C)CCO</smiles>

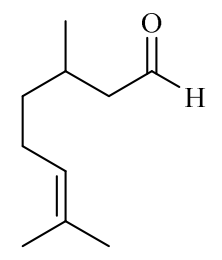

24<smiles>CC(C)=CCCC(C)=CC=O</smiles>

25

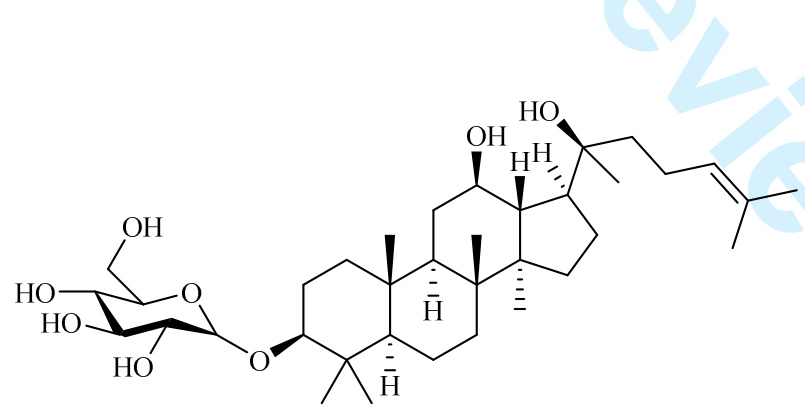

29<smiles>COC(=O)[C@@H]1[C@H]2CN3CCc4c([nH]c5cc(OC)ccc45)[C@H]3C[C@H]2C[C@H](OC(=O)c2cc(OC)c(OC)c(OC)c2)[C@H]1OC</smiles>

30<smiles>[R]C(/C=C/C(=O)N1CCCCC1)=C\c1ccc2c(c1)OCO2</smiles>

31: $\mathrm{R}=-\mathrm{H}$ 32: $\mathrm{R}=-\mathrm{CH}_{3}$ 33: $\mathrm{R}=-\mathrm{CH}_{2} \mathrm{CH}_{3}$<smiles>CCCC(C=CC(=O)N1CCCCC1)=Cc1ccc(OC)c(OC)c1</smiles>

34<smiles>COc1cc(-c2cc(=O)c3c(O)cc(O)cc3o2)cc2c1OC(c1cc[14c](O)c(OC)c1)[C@@H](CO)O2</smiles>

35<smiles>COc1c(O)c(OC)c2oc(-c3ccc(O)cc3)c(OC)c(=O)c2c1O</smiles>

36
27: $\mathrm{R}_{1}=-\mathrm{OH} \mathrm{R} \mathrm{R}_{2}=-\mathrm{CH}\left(\mathrm{CH}_{3}\right)_{2} \mathrm{R}_{3}=-\mathrm{H}$ 28: $\mathrm{R}_{1}=-\mathrm{H} \mathrm{R}_{2}=-\mathrm{CH}\left(\mathrm{CH}_{3}\right)_{2} \mathrm{R}_{3}=-\mathrm{OH}$

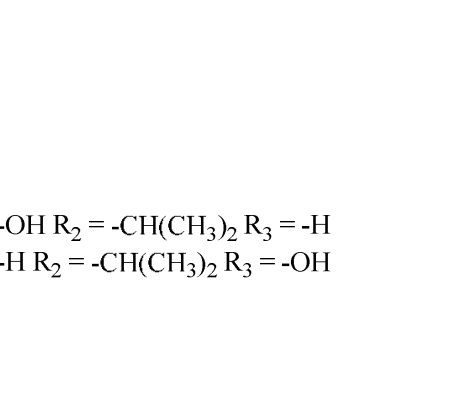
of these<smiles>[R3]c1cc2c(c([R])c1[R])CCC1C(C)(C)CCC[C@]21C</smiles>

HO<smiles>COc1ccc(-c2coc3cc(C)cc(O)c3c2=O)cc1</smiles>

Figure 5 Structures of MFS inhibitors $21-\mathbf{1 1 5}$. Ac $=$ Acetyl. 


$$
\text { Journal Name, 2014, Vol. 0, No. } 0
$$<smiles>O=c1c(-c2ccc(O)c(O)c2)coc2cc(O)cc(O)c12</smiles>

38<smiles>O=C(/C=C/c1ccc(O)cc1)OCC1C(O)OC(Oc2c(-c3ccc(O)cc3)oc3cc(O)cc(O)c3c2=O)C(O)C1O</smiles>

41<smiles>CN(C)CCOc1ccc(C(=O)/C=C/c2ccc(Oc3ccccc3)cc2)cc1</smiles>

43

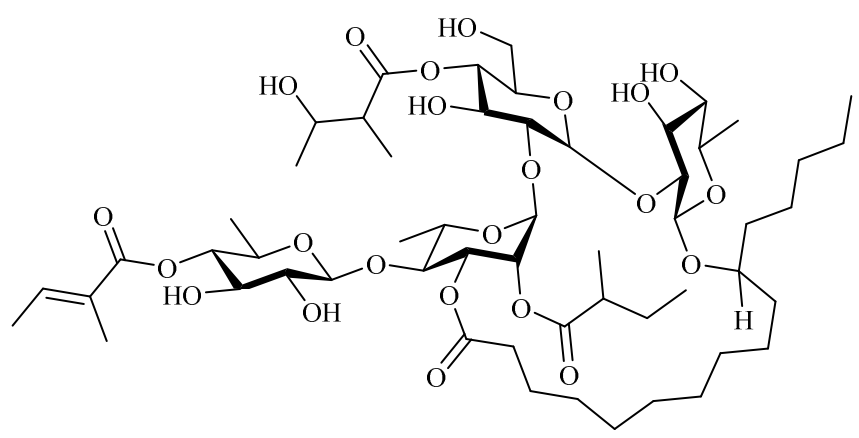

46

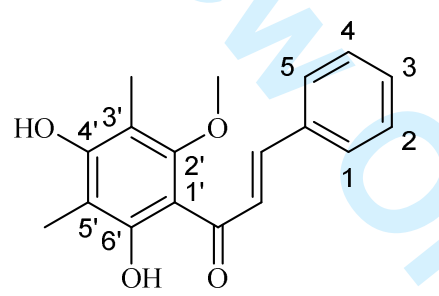

44<smiles>COc1ccc(-c2cc(=O)c3c(OC)c(OC)c(OC)cc3o2)cc1</smiles>

$39 \quad 40$<smiles>C[C@@H]1O[C@H](Oc2c(-c3ccc(O)cc3)oc3cc(O)cc(O)c3c2=O)[C@H](OC(=O)/C=C/c2ccc(O)cc2)[C@H](O)[C@@H]1OC(=O)/C=C/c1ccc(O)cc1</smiles>

42

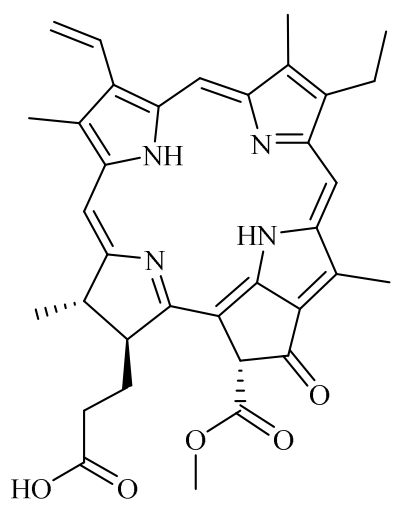

45

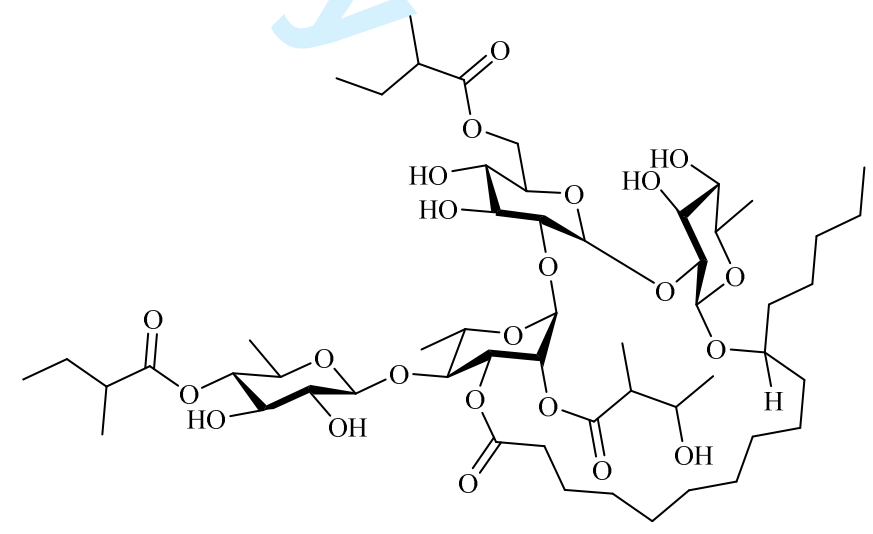

47 
Short Running Title of the Article

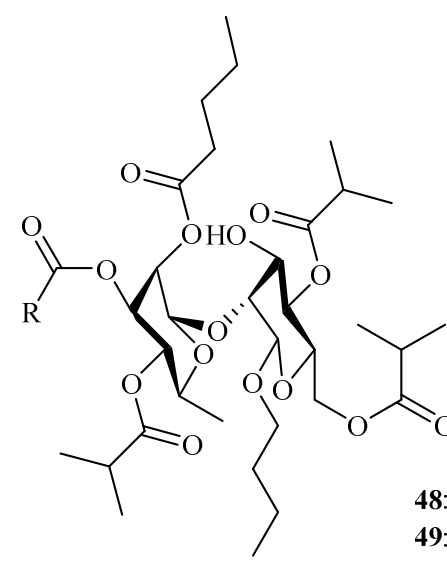

48: $\mathrm{R}=\mathrm{CH}_{3}$<smiles>COc1cc(CNC(=O)CCCC/C=C/C(C)C)ccc1O</smiles>

50

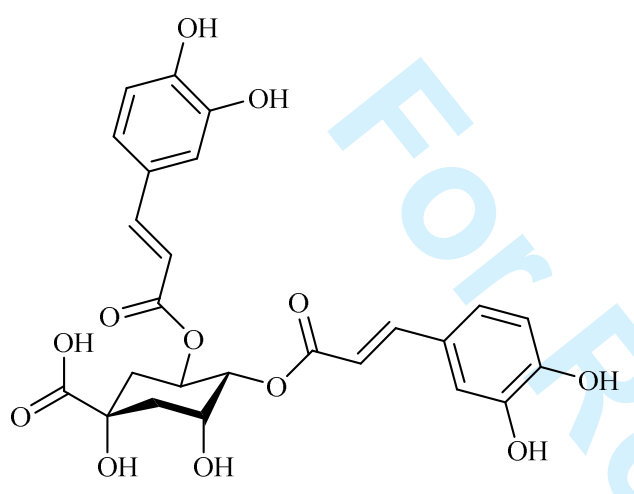

51<smiles>CC[C@H](C)C(=O)c1c(O)cc(O)cc1OC/C=C(\C)CCC=C(C)C</smiles>

52<smiles>[R]c1ccc(CCNC(=O)c2c([R])cccc2[R])cc1[R4]</smiles>

53: $\mathrm{R}_{1}=\mathrm{R}_{2}=\mathrm{R}_{3}=\mathrm{R}_{4}=-\mathrm{H}$ 54: $R_{1}=R_{2}=-O H R_{3}=R_{4}=-H$ 55: $\mathrm{R}_{1}=\mathrm{R}_{2}=-\mathrm{H} \mathrm{R}_{3}=\mathrm{R}_{4}=-\mathrm{OCH}_{3}$<smiles>COc1ccc(CCNC(=O)/C=C/c2ccc(O)c(OC)c2)cc1O</smiles>

56<smiles>COc1ccc(/C=C/C(=O)NCCc2ccc(O)c(O)c2)cc1OC</smiles>

57<smiles>CC(=CCOc1c2ccoc2cc2oc(=O)ccc12)CCC1OC1(C)C</smiles>

59<smiles>C/C(=C\COc1ccc2ccc(=O)oc2c1)CCC1OC1(C)C</smiles>

60

Figure 5 (cont.) 
<smiles>CCC(C)C(=O)c1c(O)c(CC=C(C)C)c2oc(=O)cc(-c3ccccc3)c2c1O</smiles>

61<smiles>COc1cc(/C=C/C(=O)/C=C(O)/C=C/c2ccc(O)c(OC)c2)ccc1O</smiles>

64<smiles>O=[N+]([O-])c1ccc2[nH]c(-c3ccccc3)cc2c1</smiles>

68<smiles>CC1(C)CC(=O)C2=C(C1)c1c(ccc3ccccc13)NC2c1cc(Br)ccc1O</smiles>

71<smiles>COc1ccc2c(Cc3ccccc3-c3cc4cc([N+](=O)[O-])ccc4[nH]3)c3[n+](cc2c1OC)-c1cc2c(cc1CC3)OCO2</smiles>

74<smiles>CCC(C)C(=O)c1c(O)c(CC=C(C)C)c(O)c2c(-c3ccccc3)cc(=O)oc12</smiles>

62<smiles>COc1ccc2ccc(=O)oc2c1CC=C(C)C</smiles>

63<smiles>CCOC(=O)c1c(C)nc(C)c(C(=O)OCC)c1-c1ccccc1</smiles>

65<smiles>[R]c1ccc(-c2oc3cc4c(cc3c2C=O)OCO4)c(OC)c1</smiles>

66: $\mathrm{R}=-\mathrm{OH}$ 67: $\mathrm{R}=-\mathrm{OAc}$

69<smiles>O=[N+]([O-])c1ccc2[nH]c(-c3cc(OCc4ccccc4)ccc3CO)cc2c1</smiles>

73

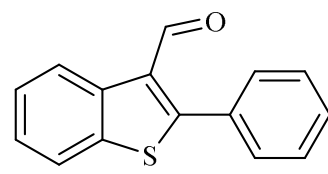

75<smiles>COc1cc(-c2cccc(C(F)(F)F)c2)sc1-c1cccnc1</smiles>

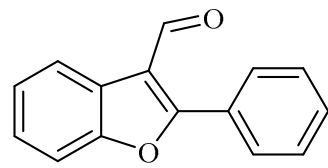

77

Figure 5 (cont.) 
Short Running Title of the Article

Journal Name, 2014, Vol. 0, No. 011

1

2

3

4

5

6

10

11

12

13

14

15

16

17

18

19

20

21

22

23

24

25

26

27

28

29

30

31

32

33

34

35

36

37

38

39

40

41

42

43

44

45

46

47

48

49

50

51

52

53

54

55

56

57

58

59

60

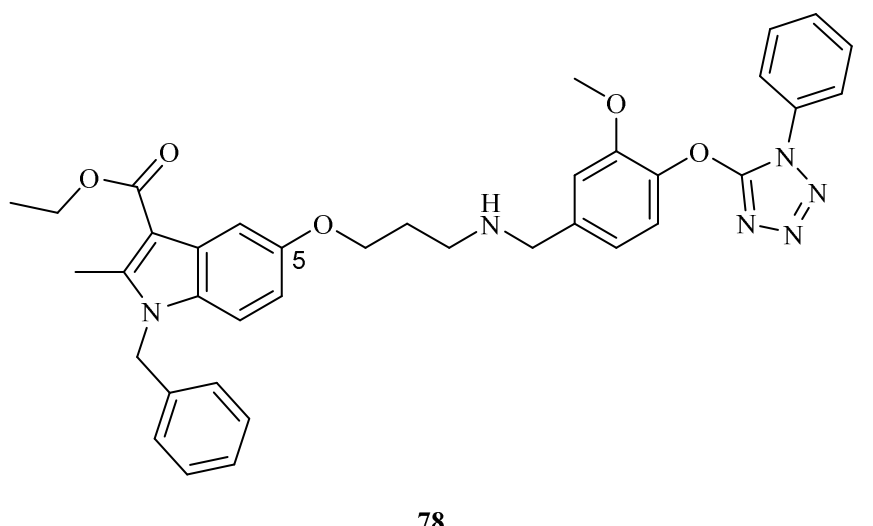

78<smiles>O=C(Nc1ccccc1)Nc1ccc(CNc2c(F)cc3c(=O)c(C(=O)O)cn4c3c2OCC4)cc1</smiles>

80<smiles>[R]N=C1C2=C(CCN1C1=C([R])c3c(c(=O)c(C(=O)OCC)cn3C3CC3)C=C(N)[R]1[H])SCC2C</smiles>

81: $\mathrm{R}_{1}=-\mathrm{OCH}_{3} \mathrm{R}_{2}=-\mathrm{CH}_{3}$

82: $\mathrm{R}_{1}=-\mathrm{OH} \mathrm{R} \mathrm{R}_{2}=-\mathrm{H}$

83: $\mathrm{R}_{1}=-\mathrm{OH} \mathrm{R}=-\mathrm{CH}_{3}$

84: $\mathrm{R}=$

85: $\mathrm{R}=$<smiles>CC(C)CCN1CCCCC1</smiles>

87: $\mathrm{R}=$<smiles>CC(C)CN(C)C</smiles>

88: $\mathrm{R}=$<smiles>CCN(CCC(C)(C)C)Cc1ccccc1</smiles><smiles>[Y]c1ccc2c(c1)C=C(/C=C/C(=O)NCC(C)C)CC2</smiles>

89<smiles>OB(O)c1ccc(OCc2ccccc2)cc1</smiles>

90: $R=N$
91: $R=C$

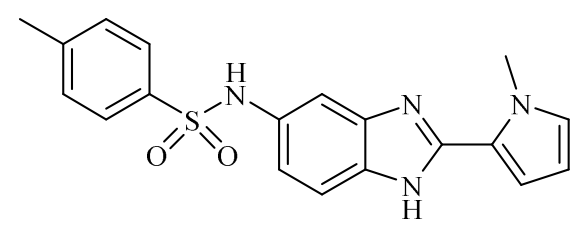

94<smiles>Cc1cc(B(O)O)cnc1OCCCc1ccccc1</smiles>

92<smiles>C[C@H](NCc1cccc(OCc2ccccc2)c1)[C@H](O)c1ccccc1</smiles>

95

Figure 5 (cont.) 
<smiles>COc1ccc2[nH]c(S(=O)Cc3ncc(C)c(OC)c3C)nc2c1</smiles>

96<smiles>CCN(CC)CCSc1nc2ccsc2n2cccc12</smiles><smiles>CC(=O)c1c(-c2ccc(F)cc2)cn(-c2ccc(C)cc2)c1C</smiles>

102<smiles>Fc1ccc([C@H]2CCNC[C@H]2COc2ccc3c(c2)OCO3)cc1</smiles>

104<smiles>Fc1ccc([C@@H]2CCNC[C@H]2COc2ccc3c(c2)OCO3)cc1</smiles>

105<smiles>COc1ccc2[nH]c(S(=O)Cc3nc4cc(Cl)ccc4n4cccc34)nc2c1</smiles>

97<smiles>COc1ccc2c(c1)nc(SCCN1CCCC1)c1cccn12</smiles>

98<smiles>COc1cc(C(=O)C(=O)N(C)[C@@H](Cc2ccc(Cl)cc2)C(=O)N(Cc2ccccc2)C(CCc2ccncc2)Cc2ccncc2)cc(OC)c1OC</smiles>

101<smiles>COc1cc2c(cc1OC)CN(CCc1ccc(NC(=O)c3cc(OC)c(OC)cc3NC(=O)c3cnc4ccccc4c3)cc1)CC2</smiles><smiles>COc1ccc(OC[C@@H]2CN(C)CC[C@H]2c2ccccc2)cc1</smiles>

106<smiles>Fc1ccc([C@H]2CCNC[C@H]2COc2cc(Br)ccc2Cl)cc1</smiles>

107<smiles>Fc1ccc(C2CCNC[C@H]2/C=C/c2cc(Br)ccc2Cl)cc1</smiles>

108<smiles>Cc1ccc(-c2cc(C(F)(F)F)nn2-c2ccc(S(N)(=O)=O)cc2)cc1</smiles>

109<smiles>O=[N+]([O-])c1ccc(-n2nc(-c3ccc(Cl)cc3)c3c2-c2ccccc2S(=O)(=O)N3)cc1</smiles>

110

Figure 5 (cont.) 


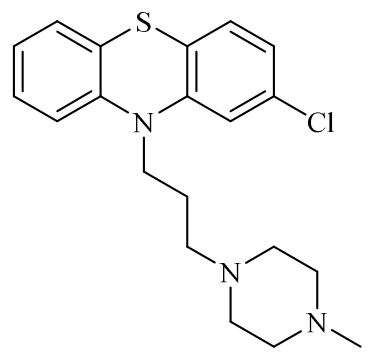

111

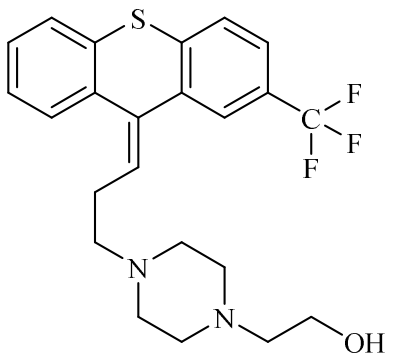

112

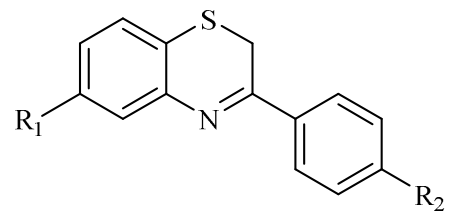

113: $\mathrm{R}_{1}=-\mathrm{H} \mathrm{R}_{2}=-\mathrm{OCH}_{2} \mathrm{CH}_{3} \mathrm{CH}_{3}$ 114: $\mathrm{R}_{1}=-\mathrm{CF}_{3} \mathrm{R}_{2}=-\mathrm{NO}_{2}$<smiles>CSc1ccc2c(c1)N(CCC1CCCCN1C)c1ccccc1S2</smiles>

115

Figure 5 (cont.)

compounds were carried out. Monoterpenes nerol (21), estragole (22), and dimethyl-octanol (23) were tested against $S$. aureus. Compounds $\mathbf{2 1}$ and $\mathbf{2 3}$ were able to enhance the activity of norfloxacin, decreasing its MIC against the NorA overexpressing strain while not displaying intrinsic antibacterial activity. These open chain terpenes proved to be more effective than 22, which carried a benzene ring, at inhibiting the NorA pumps [58].

Citronellal (24) and citral (25) are monoterpenes, and were chosen as starting material for the synthesis of new amide derivatives. The basis for this study was the fact that aromatic amides are recognized as potent EPIs, whereas alkyl, alkenyl, and alkynil amides had not yet been explored. The compounds obtained were 5,9-dimethyldeca-2,4,8trienoic acid amides and 5,9-dimethyldeca-2,8-dienoic acid amides, whose activity for the potentiation of ciprofloxacin in $S$. aureus was assessed. Of the first 22 synthesized compounds, seven were able to reduce ciprofloxacin's MIC by 4 -fold. Further modifications were performed, and the $\mathrm{C}$ 9 methyl group was replaced by an amide, yielding compounds with similar potency as the previous ones. Derivatives subjected to hydrogenation led to decreased activity, which reveals that unsaturation is an important feature for antimicrobial potentiation. The mechanism of inhibition was assessed through ethidium bromide efflux, confirming these compounds act by inhibiting NorA, being compound 26, a 9-formyl-5-methyldeca-2,4,8-trienoic acid amide, the most potent inhibitor [59].

Ferruginol (27), a diterpene, showed efficiency in inhibiting the efflux of ethidium bromide, proving its activity as a NorA inhibitor. It also showed a decrease in norfloxacin efflux in S. aureus [60]. Totarol (28), a phenolic diterpene, also showed a reduction in ethidium bromide efflux, suggesting activity against NorA pump, even though its mechanism is not yet known [61].

The triterpene ginsenoside 20(S)-Rh2 (29), a trace compound from red ginseng, also enhanced the effect of ciprofloxacin against $S$. aureus, through a NorA-mediated efflux inhibition, promoting the antibiotic accumulation in the bacteria [62].Reserpine (30) is a natural alkaloid derived from Rauwolfia vomitoria, known as an anti-hypertensive agent, with inhibitory activity against human P-gp and breast cancer resistance protein (BCRP) [63-64]. Reserpine (30) has proven to be an effective inhibitor of the Bmr multidrug transporter in Bacillus subtilis, diminishing the efflux of ethidium bromide. Its structural analogue rescinnamine also had EPI activity, in a smaller extension than reserpine (30) [65]. Through DNA sequencing of the $b m r$ genes of mutants, it was shown that reserpine (30) interacts with the residues Phe-143, Val-286, and Phe-306 of Bmr pumps, inhibiting drug transport [19]. Compound $\mathbf{3 0}$ has shown potential as an EPI for the inhibition of NorA efflux pumps in $S$. aureus, which are structurally related to Bmr pumps, reversing susceptibility to fluoroquinolones, demonstrating reductions up to 4-fold in MIC for some tested strains. It has been shown that 30's effect is more pronounced in hydrophilic fluoroquinolones, such as ciprofloxacin [66]. The mechanism by which $\mathbf{3 0}$ performs the inhibitory activity is through direct binding to the transporters that mediate drug efflux, acting as a competitive inhibitor [19,67]. Reserpine (30) also succeeded in inhibiting the chloramphenicol efflux by CmlR1 and CmlR2 of Streptomyces coelicolor, the ciprofloxacin and norfloxacin efflux in Listeria monocytogenes's pump Lde, and the kanamycin and fusidic acid efflux by LmrS from a MRSA clinical isolate. On the other hand, for L. monocytogenes, $\mathbf{3 0}$ was not able to reverse susceptibility to linezolid and lincomycin, which suggests that 30 does not completely overlap with the structure binding sites of efflux pumps, being the inhibition dependent on the type and nature of the substrate [19]. However, reserpine (30) shows neurotoxicity at the concentration required to inhibit NorA, leading to the need to investigate new inhibitors [67].Piperine (31), an alkaloid found in the fruits of Piper nigrum, was found to be an inhibitor of ciprofloxacin efflux in $S$. aureus, reducing the MIC by 2fold. It is thought that compound $\mathbf{3 1}$ acts as a direct inhibitor of drug efflux. Compound $\mathbf{3 1}$ inhibits drug transport of the pump MdeA, potentiating the effects of benzalkonium chloride, doxorubicin, daunorubicin, novobiocin, tetraphenylphosphonium chloride, rhodamine $6 \mathrm{G}$, virginiamycin and mupirocin [17, 19-20]; it also inhibits ciprofloxacin efflux by NorA [68]. 
Given these positive results obtained with piperine (31), further research into this scaffold was performed. Kumar et al. (2008) performed a screening of about 200 structurally diverse compounds for the inhibition of NorA efflux pump, choosing ethidium bromide as a substrate, since the only mechanism through which this compound is expelled is active transport (68). About 50 compounds were able to increase the intracellular concentration of ethidium bromide by 4 -fold, while not displaying antibacterial activity. Five of these compounds were more potent than piperine (31), being three of these compounds selected for further studies: 32, 33, and $\mathbf{3 4}$, being 32 and $\mathbf{3 4}$ more potent than $\mathbf{3 1}$ by 40 -fold [18, 68]. Assumptions regarding structure-activity relationship (SAR) studies of these molecules could be drawn (Figure 6). specific microbial MDR inhibitor, as flavonoids with P-gp inhibitor properties usually possess alkylated 7-O groups. In fact, when added to a wild type strain of $S$. aureus, compound 35 caused a decrease in norfloxacin's MIC to $0.25 \mu \mathrm{g} / \mathrm{ml}$. Furthermore, when tested with berberine and palmatine, 35 appeared to completely inhibit NorA, suggesting it acts as a non-competitive inhibitor [71].

A number of hydnocarpin-type flavolignanes were synthesized in order to perform SAR studies. Flavolignanes with and without phenolic groups at the 5 and 7 positions had comparable activity. Diosmetin (11), the parent compound, presents hydroxyl groups in the A ring and was the most potent compound. Peracetate derivatives of $\mathbf{3 5}$ have approximately the same potency as their parent compounds,

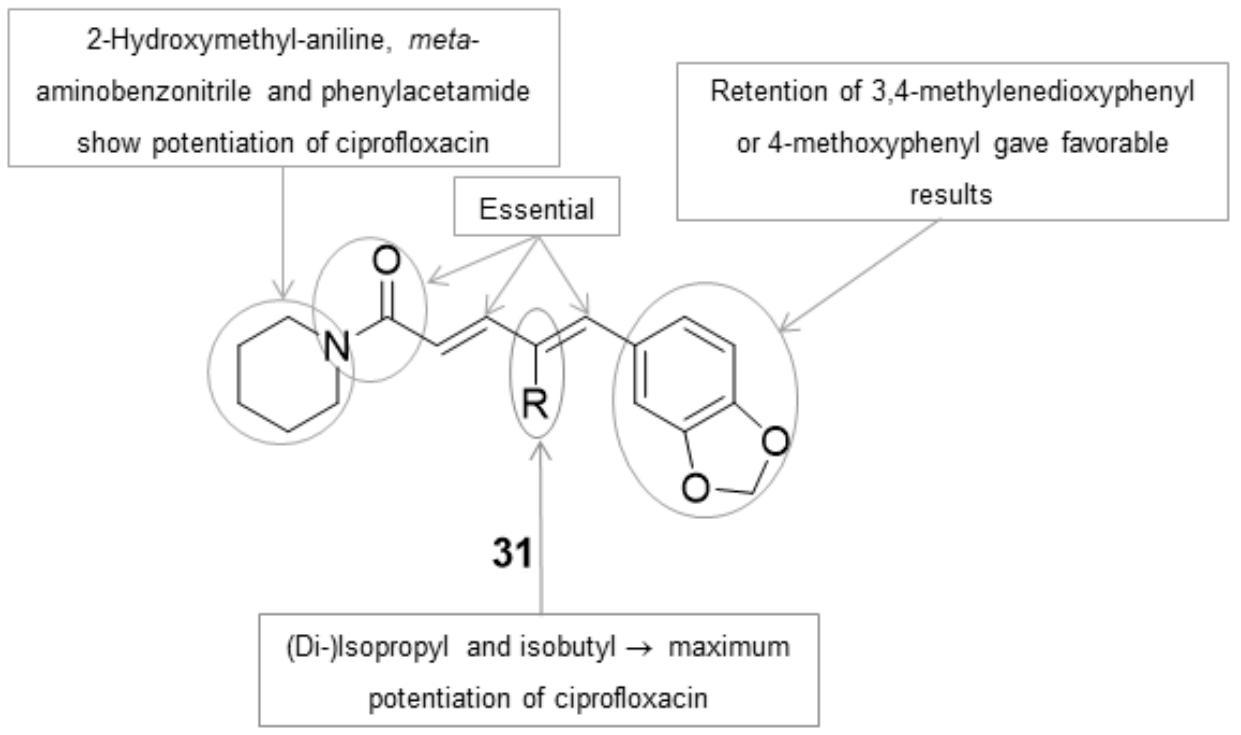

Figure 6 Conclusions from SAR studies for piperine and its derivatives.

It was demonstrated that the introduction of an alkyl group at C-4 position, mainly an isopropyl, isobutyl or di-isopropyl group, coupled with the substitution of the piperidinyl moiety with an aromatic amine, such as aniline, would lead to maximum potentiation of ciprofloxacin; if the anilinyl moiety is substituted with a 2-hydroxymethyl group or a nitrile, regardless of the positional isomery, ciprofloxacin activity would increase. Replacing the piperidinyl moiety with a phenylacetamide yields good potentiation of ciprofloxacin, reducing its MIC by 4-fold, regardless of the isomery. Unsaturation is crucial for the potentiation, as well as the amide. Lastly, the retention of 3,4methylenedioxyphenyl or 4-methoxyphenyl groups in the 1,3-benzodiol moiety also gives favorable results [68-70]. Plants of the Berberis species have the ability to produce the alkaloid berberine, which is a plant secondary metabolite produced in response to microbial invasion, whose activity is enhanced by a flavolignane also produced by these species, 5'-methoxyhydnocarpin (35) [71]. Compound 35 exists in a mixture of anion and neutral compound at physiological $\mathrm{pH}$, since it has a phenolic group with a $\mathrm{pKa}$ of 7.3 . Due to the acidic properties of this $7-\mathrm{OH}$ group, 35 is likely to be a and could be considered prodrugs, if deacetylated by $S$. aureus, which seems unlikely. Considering the D ring, compounds bearing a 3"'-methoxy-4"'-hydroxy moiety displayed good activity, and 3', 5',-dimethoxy-4"'-hydroxy moieties conferred a slightly less active compound. As for the $\mathrm{B}$ ring, the presence of an $\mathrm{H}$ or an $\mathrm{OH}$ is not critical for activity [72]. In addition, a flavone isolated from Alkanna orientalis, sarothrin (36), blocked ethidium bromide efflux by $S$. aureus, suggesting its potential activity as a NorA inhibitor [73].

Biochanin A (37), an isoflavone, also showed potentiation of norfloxacin and berberine against wild-type $S$. aureus and Bacillus megaterium. An analogue of luteolin, orobol (38), was quite active as a NorA inhibitor, unlike luteolin itself. It was shown that flavones which have a single methoxy in its B-ring are more potent than flavones with two methoxy groups in the B-ring [74].

Another flavone, baicalein (39), was capable of potentiating the effect of ciprofloxacin in $S$. aureus. Studies aimed at measuring the efflux of ciprofloxacin and pefloxacin, a poor NorA substrate, were performed, and showed that compound 39 was much more potent at restoring ciprofloxacin 
sensitivity than pefloxacin, leading to the conclusion that this flavone acts as a NorA efflux inhibitor, even though its mechanism remains unknown [75].

Maia et al. (2011) identified six flavonoids from the plant Praxelis clematidea: apigenine, genkwanin, 7,4'dimethylapigenin, trimethylapigenin, cirsmaritin, and tetramethylscutellarein (40) [76]. These methoxylated flavones showed no antibacterial activity, but were able to reduce the MIC of norfloxacin at least by 2 -fold and up to 16-fold, as was the case of $\mathbf{4 0}$, at a concentration of $8 \mu \mathrm{g} / \mathrm{ml}$, being therefore considered NorA efflux pump modulators. It is thought that the lipophilicity of the flavonoids, strengthened by the presence of methoxyl groups, is responsible for this activity [21]. Presence of a methoxy in the 4' position proved to be decisive for activity, as flavonoids containing this group were more potent. The number of methoxy groups is also essential for potency, being the most metoxylated compounds the most active [76]. Tiliroside (41), an amphipathic kaempferol glycoside, reduced the concentration of antibiotic needed to inhibit the growth of bacteria. The lipophilicity of the flavone moiety may be a determinant factor for the activity displayed. Tiliroside (41) showed no antibacterial activity against $S$. aureus, but showed a decrease in the MIC of fluoroquinolones up to 16 -fold, when at a concentration of $64 \mu \mathrm{g} / \mathrm{ml}$. The reduction in the MIC of biocides, such as ethidium bromide and benzalkonium chloride, was impressive, with the MIC of acriflavine showing a decrease by 128 -fold at the concentration of $64 \mu \mathrm{g} / \mathrm{ml}$ and $32 \mu \mathrm{g} / \mathrm{ml}$. These results suggest that $\mathbf{4 1}$ might additionally act on pumps other than NorA [77].

Kaempferol 3-O- $\alpha$-L-(2, 4-bis- $E$ - $p$-coumaroyl)rhamnoside (42) was also able to potently inhibit in a concentration dependent manner NorA-mediated ethidium bromide efflux in $S$. aureus. This compound also increased the antibiotic effect of norfloxacin against a wild-type and a NorA overexpressing strain of $S$. aureus. However, it has to be considered that $\mathbf{4 1}$ and $\mathbf{4 2}$ are flavonoid glycosides and cinnamoyl esters, and that their activity can become compromised with the activity small intestine $\beta$-glucosidases or plasma esterases, even though the activity might possibly lie in the kaempferol moiety, with 41 and 42 acting as prodrugs. The authors hypothesized that if the activity of compound $\mathbf{4 2}$ is compromised, this compound could be used as adjuvant of ciprofloxacin for topical infections [78].

Since 42 showed promising results as a NorA EPI, the same group performed a screening of a library of 117 chalcones, due to their structural similarities with the coumaroyl substituents [79]. Ten out of the 117 screened chalcones presented medium efflux inhibition at $20 \mu \mathrm{g} / \mathrm{ml}$. The most potent chalcones, such as $\mathbf{4 3}$, bear a dimethylaminoethoxy moiety, which might disrupt the proton gradient, thus eliminating the energy source of NorA. Five of these chalcones possessed a hydroxyl group at the 4'-position, and potentiated the activity of berberine against $S$. aureus and $B$. cereus, suggesting that this substituent is also important for activity. The compound with a methoxy group at position $\mathrm{C}$ 2 has shown to present the best activity. The majority of chalcones tested showed a 4-fold reduction of ciprofloxacin's MIC in $S$. aureus, suggesting the presence of a NorA efflux pump inhibitory activity. Compound $\mathbf{4 3}$ showed 8-fold and 16-fold reduction of the MIC of ciprofloxacin at 6.25 and $12.5 \mu \mathrm{g} / \mathrm{ml}$, respectively. However, it also showed antibacterial activity at $25 \mu \mathrm{g} / \mathrm{ml}$. Some chalcones also presented antiparasitic activity, namely against Leishmania major and Plasmodium yoelii [79].

Another chalcone, 4',6'-dihydroxy-3',5'-dimethyl-2'methoxychalcone (44), showed good activity as a MDR, causing complete growth inhibition at a very low concentration $(3.3 \mu \mathrm{g} / \mathrm{ml})$ when combined with a sub inhibitory concentration of berberine. When tested in combination with berberine, erythromycin, and tetracycline against strains of $S$. aureus, there was an increase in the activity of all tested compounds in the wild-type $S$. aureus, indicating a mode of action likely related to the NorA pump. This compound was also tested against $B$. cereus, potentiating the effects of the tested antibiotics as well, and displaying a favorable effect, particularly in the case of berberine, where a 30-fold increase in the activity was observed [80].

Pheophorbide A (45) is an intermediate of the natural breakdown of chlorophyll. Concentrations as low as 0.5 $\mu \mathrm{g} / \mathrm{ml}$ of pheophorbide A with sub-inhibitory concentrations of berberine were enough to completely inhibit $S$. aureus growth. When extended to fluoroquinolones, this compound also proved efficient in potentiating the effect of norfloxacin by 4-fold in a wild-type strain, being its action directed towards NorA [81].

Orizabins, oligosaccharides from resin glycosides, were evaluated for their efflux pump inhibitory activity on strains of $S$. aureus. These amphipathic compounds showed synergy with norfloxacin, whilst showing no antibacterial activity by themselves. Orizabin IX (46) completely inhibited the growth of a NorA overexpressing strain at $1 \mu \mathrm{g} / \mathrm{ml}$, while orizabin XIX (47) reversed norfloxacin resistance by 4 -fold at $25 \mu \mathrm{g} / \mathrm{ml}$ for the same strain [82]. Murucoidins, which are also resin glycosides, strongly potentiated the action of norfloxacin against a $S$. aureus NorA overexpressing strain by 4 -fold at concentrations ranging from 5 to $25 \mu \mathrm{g} / \mathrm{ml}$. Stoloniferin I potentiated the same activity by 8 -fold at a concentration of $5 \mu \mathrm{g} / \mathrm{ml}$ [83].

A study aiming to find new EPIs from the plant Geranium caespitosum led to the isolation of novel acylated neohesperidosides. It was found that two compounds, compound 48 and 49, were successful at restoring susceptibility to berberine, norfloxacin, and ciprofloxacin at sub inhibitory concentrations, possibly due to NorA inhibition [84].

Capsaicin (50), the major constituent of the fruits of the Capsicum genus, is a known P-gp inhibitor. Studies were performed, in order to evaluate if its inhibitory activity was also applied to MDR pumps in bacteria. In a strain of $S$. aureus overexpressing the NorA efflux pump, and therefore resistant to fluoroquinolones, $\mathbf{5 0}$ proved efficient in increasing the susceptibility to ciprofloxacin, while reducing the emergence of ciprofloxacin-resistant mutants. Molecular 
modelling studies of the complex capsaicin (50)-NorA, using the glycerol-3-phosphate transporter to predict the structure of NorA, showed that compound $\mathbf{5 0}$ aliphatic chain extended into NorA's hydrophobic cleft. Furthermore, a weak hydrogen bond between Arg-98 and the aryl hydroxyl contributed to the stability of the complex [85].

Studies in the aqueous extract of Artemisia absinthium showed promise in the inhibition of MDR efflux pumps. It was proven that this activity came from caffeoylquinic acids. At a concentration of $20 \mu \mathrm{M}, 4$ '-5'-O-dicaffeoylquinic acid (51) potentiated the activity of berberine by 16-fold and of norfloxacin, by 10 -fold against a wild-type strain of $S$. aureus, while not displaying effects in a nor $A$-deleted strain, confirming this compound's NorA inhibition ability [86]. Olympicin A (52) is an acylphloroglucinol, found in the aerial parts of Hypericum olympicum. This molecule had some efflux impeding ability, proven by the improvement of intracellular accumulation of enoxacin in a NorA overexpressing strain of $S$. aureus, at $50 \mu \mathrm{g} / \mathrm{ml}$ [87].

Riparins are natural alkylamides found in the plant Aniba riparia. Since the extraction of these compounds comes with very low yields, synthetic approaches were developed. As such, the fundamental core of riparins, Rip-A (53), was synthesized and derivatives were prepared, Rip-B (54) to E (55). Rip-E (55) showed good growth inhibitory activity against $S$. aureus, attributed to their lipophilic nature and the presence of hydroxyl groups at the benzamide moiety.
Extracts of Mirabilis jalapa have shown good results in reversing fluoroquinolone resistance in strains of $S$. aureus overexpressing the NorA efflux pump. One compound in particular, $\quad \mathrm{N}$-trans-feruloyl-4'-O-methyldopamine (56), caused an 8-fold reduction in the MIC of norfloxacin, making it a potential lead in the search for structurally similar synthetic alkylamines [89].

Synthesis of derivatives was undertaken, taking $N$-transferuloyl-4'-O-methyldopamine (56) as a model. The aim was to identify active compounds from natural sources and, as such, couplings between cinnamic acid derivatives and natural-occurring amines were chosen. SAR criteria was taken into account, as it is known that substitution of the aromatic rings, methoxy or hydroxyl substitution, double bonds and aromatic ring nature in the amine part influence the activity. The lead compound was $\mathrm{N}$-trans-3,4-Odimethylcaffeoyl dopamine (57) since it showed the same activity as the natural compound. It was found that, for the cinnamic moiety, a hydroxyl substitution on the aromatic ring appears to be better than a methoxy group or unsubstituted derivatives and the double bond is essential for activity. As for the amine part, trisubstitution on the aromatic ring increases antibacterial activity, at the cost of decreased efflux pump inhibition. Methoxy substitution yields compounds with better results than hydroxyl substitution, which was better than no substitution and tryptamine combinations showed the best results (Figure 7). Among the

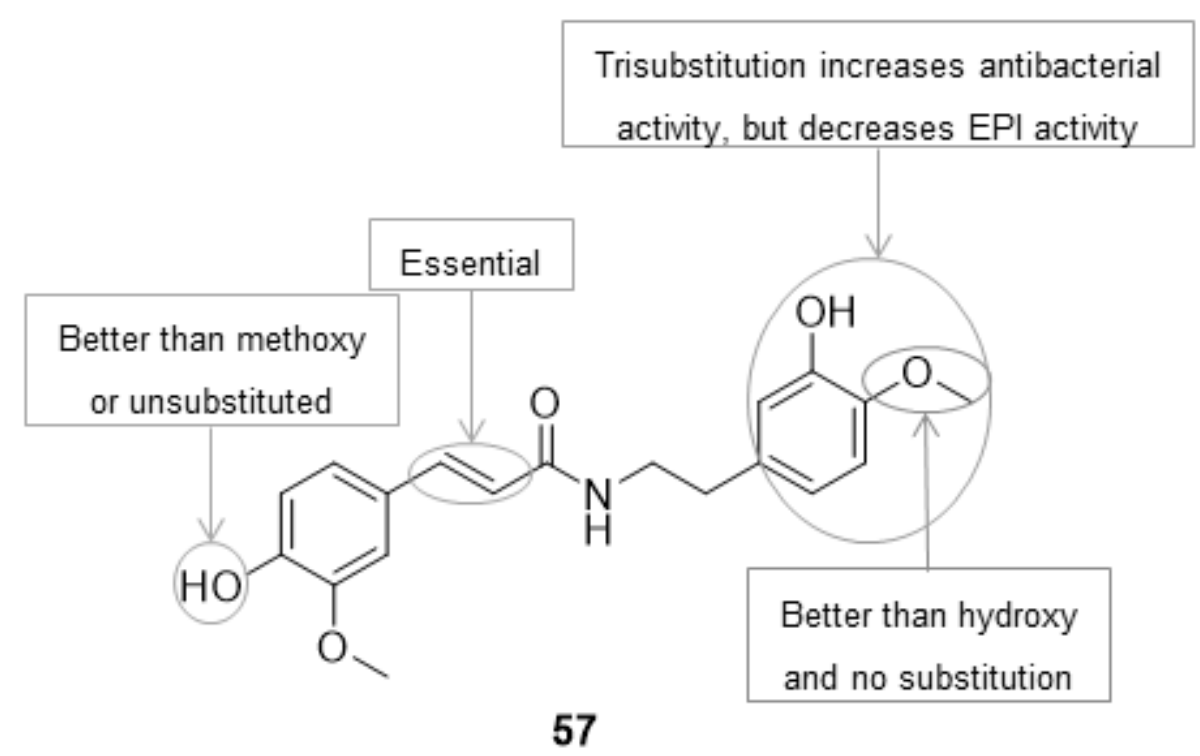

Figure 7 Conclusions from SAR studies for compound $\mathbf{5 7 .}$

Oppositely, Rip-B (54), with two methoxy groups at the phenyl-ethyl moiety did not present antibacterial activity, presenting instead a decrease in the MIC of ciprofloxacin and norfloxacin, similarly to reserpine. Combined with the ability of decreasing ethidium bromide's MIC, the activity of compound $\mathbf{5 4}$ was attributed to NorA inhibition, suggesting its potential as an EPI [88]. compounds tested, compound $\mathbf{5 8}$ showed potentiation of norfloxacin comparable to that of reserpine (30). At a concentration of $30 \mu \mathrm{M}, \mathbf{5 8}$ showed good results for the inhibition of ethidium bromide efflux. For these compounds, it was shown that the activity reaches its highpoint when the phenyl ring is substituted with two hydroxyls [89].

Grapefruit oil fractionation led to the isolation of three compounds, two coumarin derivatives and one bergamottin 
derivative. The bergamottin epoxide (59) showed a reduction of ethidium bromide's MIC by 6-fold on different MRSA strains, and a 20-fold reduction in norfloxacin's MIC. This reduction was also achieved when the coumarin epoxide (60) was present, and suggests an activity towards NorA efflux pumps [90].

Coumarins isolated from Mesua ferrea were also studied for their efflux pump inhibitor activity against clinical strains and NorA overexpressing strains of $S$. aureus. Seven coumarins were tested, and showed good results in inhibiting ethidium bromide efflux. Two compounds, 61 and 62 , showed potential EPI activity against the NorA overexpressing strain and a MRSA strain, using norfloxacin as a substrate, which makes them desirable compounds for the synthesis of derivatives [91].

Screening of natural products with P-gp inhibitory effects $(92,93)$ led to the identification of two compounds with NorA inhibitory activity, osthol (63) and curcumin (64) [36, 92-93]. Curcumin (64), derived from the rhizomes of Curcuma longa, has additionally already shown activity as an anti-inflammatory, antioxidant, antiviral, and anticancer agent [36, 92-93]. These compounds were effective in reducing the MIC of ciprofloxacin by 4- and 8-fold, respectively [94].

A penta-substituted pyridine, 2,6-dimethyl-4-phenylpyridine-3,5-dicarboxilic acid diethyl ester (65), was isolated from the rhizomes of Jatropha elliptica. This compound, when in association with ciprofloxacin, showed a strong effect against NorA efflux pumps in S. aureus [95].

A methanol extract of the plant Dalea spinosa yielded the isolation of six compounds, whose EPI potential was analyzed against wild-type $S$. aureus and mutant strains. It was shown that spinosan A (66), at a concentration of 48 $\mu \mathrm{g} / \mathrm{ml}$, and its acetate 67 , at a concentration $42 \mu \mathrm{g} / \mathrm{ml}$, were able to decrease berberine's MIC by 8 - and 62-fold, respectively, against the wild-type. Other compounds were also able to reduce berberine's MIC, but to a lesser extent. Compound 67 also managed to decrease berberine's MIC in the NorA overexpressing strain, suggesting inhibitory activity in this efflux pump [96].

\subsubsection{Synthetic Compounds}

The INF series (compounds 68-72) resulted from an initial screening of 9600 structurally diverse compounds, in order to find NorA inhibitors for B. subtilis, not expressing Bmr. The NorA substrate chosen was ethidium bromide [67]. Since this screening did not focus on a specific chemical group, the most active compounds were divided into several groups. Several indole derivatives were active, which did not come as a surprise, since reserpine (30) itself has an indole moiety. Another large group was the trichloromethylaminal containing compounds, which were not further explored as their likelihood to be toxic was high. Biphenyl urea derivatives were also active, and other compounds, with no obvious similarities, showed activity as well. Five compounds were selected for further tests, INF 55 (68), INF 240 (69), INF 271 (70), INF 277 (71) and INF 392 (72).
These compounds were active at concentrations of $5 \mu \mathrm{g} / \mathrm{ml}$ or less, and included the most potent compound, 72, the most potent indole, 68, and the most potent biphenyl urea, 71. Derivative $\mathbf{7 2}$ was able to reduce resistance to ethidium bromide and ciprofloxacin by 8 -fold at a concentration of 0.4 $\mu \mathrm{g} / \mathrm{ml}$. All five inhibitors were more potent than reserpine (30) at promoting the activity of ciprofloxacin in $S$. aureus and, by being structurally different, it was hypothesized that a potent, nontoxic lead would be identified. These compounds also proved effective in inhibiting the Bmr multidrug transporter in B. subtilis and two compounds, 68 and 71, also enhance fluoroquinolone activity in Streptococcus pneumoniae [67].

The results obtained with INF 55 (68) in $S$. aureus as an inhibitor of the NorA efflux pump led to an increased interest in this kind of scaffold. SAR studies regarding the C5 proved that substitution in this carbon is crucial for activity. Substitution with a nitrile group leads to retention of potency, and carbonyl based electron-withdrawing groups at C-5 would result in molecules with no activity [97]. Three dimensional-quantitative structure-activity relationship (3DQSAR) studies predicted that 2-aryl indole derivatives would be NorA inhibitors. Functionalized INF 55 (68) showed a decreased MIC for berberine, a MDR transporter substrate. Berberine is an amphipathic cation alkaloid. From the series of 2-aryl-5-nitroindoles synthesized by Samosorn et al. (2009), the alcohol $\mathbf{7 3}$ was the most effective in inhibiting berberine efflux, as well as other antibiotics, such as ciprofloxacin, in S. aureus [98]. Later, the same group developed a strategy that consisted in a molecule presenting dual action: an antibiotic and a MDR inhibitor. Therefore, they combined berberine, a hydrophobic cation that is a MDR substrate, with INF 55 (68), a MFS inhibitor. These efforts yielded compound 74 [17-18, 99]. In terms of antibacterial activity in $S$. aureus, the hybrid was about 100 times more active than berberine alone, and the difference was even higher when tested against a NorA overexpressing strain of S. aureus. Enterococcus faecalis also presented resistance to berberine, but was susceptible to 74. [99] Synthesis of sulfur derivatives was achieved based on the premise that the activity of $\mathbf{6 8}$ is less dependent on the nitro group than the 2-arylindole. Benzothiophene, thiophene, and benzofuran derivatives, such as compounds $\mathbf{7 5}, \mathbf{7 6}$, and $\mathbf{7 7}$, respectively, were able to synergize with ciprofloxacin and ethidium bromide, increasing their intracellular concentration and leading to the conclusion that these compounds inhibit NorA in S. aureus. [100]

Taking into account the fact that the indole $\mathrm{NH}$ is not essential for efflux pump inhibition, Samosorn et al. (2009) simplified the indole moiety of berberine-based hybrids, along with the effect of a methylene ether linking group. Their results showed that a hybrid molecule with oxygen in the linkage chain had stronger antibacterial and MDR pump inhibitory activity than the original 74 [101]. On the other hand, removal of the indole moiety produced compounds with lower dual activity, and this structural simplification led to compounds with low antibacterial activity, but with retained NorA pump inhibitory activity. These data suggest 
that while the indole moiety is not necessary for NorA pump inhibition, it is required for the antibacterial activity of hybrid compounds [101].

A recent study identified a novel class of NorA EPIs with a polysubstituted indole moiety. Twenty molecules were highly active in inhibiting ethidium bromide efflux in $S$. aureus. However, the best results were obtained with compounds $\mathbf{7 8}$ and 79, which were derivatives that presented three and four carbon atom chains, respectively. Compound 78 presented the best half maximal inhibitory concentration $\left(\mathrm{IC}_{50}\right)$, with a concentration of $1.8 \mu \mathrm{M}$. Compound 79 exhibited similar $\mathrm{IC}_{50}(2 \mu \mathrm{M})$ and ethidium bromide inhibition as 78. Compounds with shorter or longer carbon chains did not present such good results, leading to the conclusion that three or four carbons make up the right distance between the indole scaffold and the basic center, thus presenting the best inhibitory effect. When tested for their synergy with ciprofloxacin, both $\mathbf{7 8}$ and $\mathbf{7 9}$ were able to restore the antibiotic's activity [102]. It was found that the substitution of the C-5 in the indole results in a potent EPI,
MepA pump systems from the MATE family [17-18, 104]. Fluoroquinolones containing a thiopyranopyridine moiety at the C-7 position were synthesized and their activity was tested against strains of $S$. aureus. However, these derivatives did not seem to be NorA substrates. Further investigations into thiopyranopyridinylquinolone esters were carried out. Some of the synthesized compounds displayed better inhibitory activity than reserpine (30), namely quinolone esters 81, 82 and $\mathbf{8 3}$, and showed significant MIC reduction of ciprofloxacin. Compounds $\mathbf{8 2}$ and 83 also showed a 32-fold MIC reduction of ciprofloxacin for strains of $S$. aureus overexpressing MepA pumps from the MATE family $[18,105]$. In terms of established SAR, these compounds definitely do not need the fluorine atom at the C6 position for the antibacterial activity of thiopyridinylquinolones. A methyl at the C-8 position yields a compound with enhanced activity against Gram-positive bacteria for 6-amino derivatives. The thiopyranopyridine moiety at the C-7 position is crucial for NorA and MepA inhibition (Figure 8) [105].

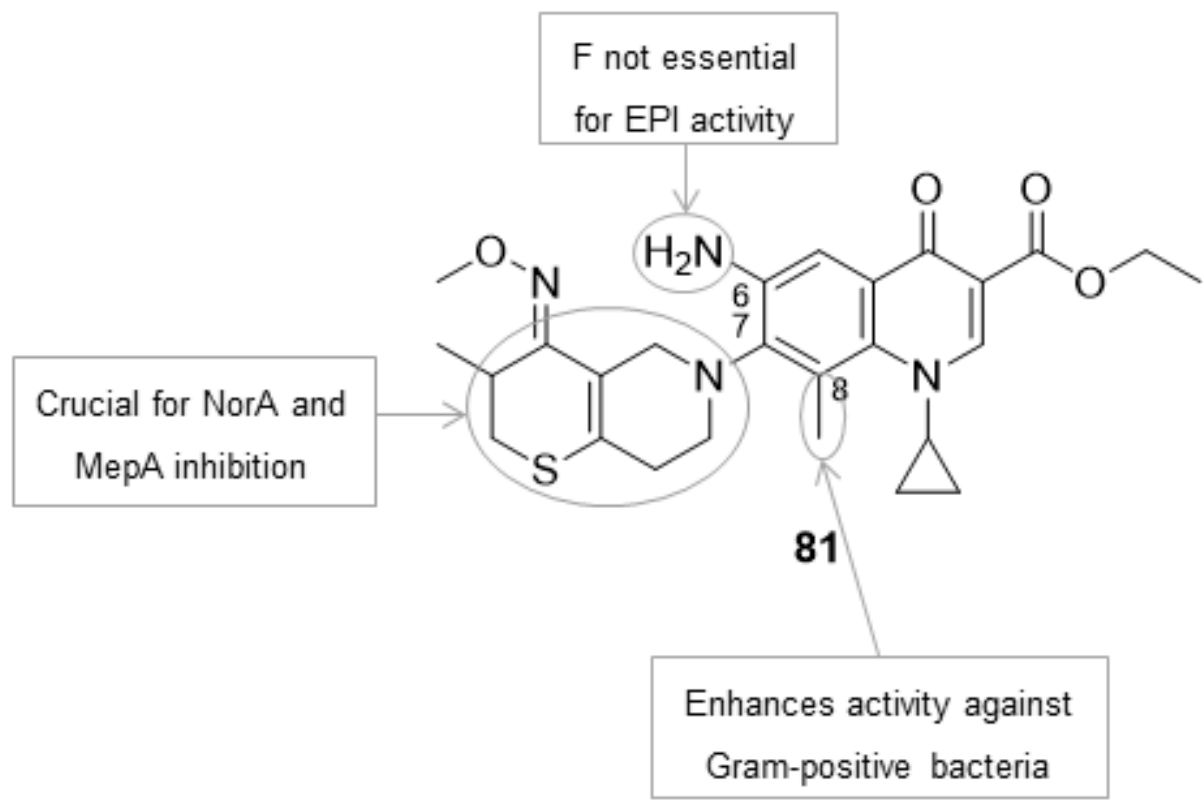

Figure 8 Conclusions from SAR studies for fluoroquinolone $\mathbf{8 1}$ derivatives.

particularly if there is a propoxyl chain carrying terminal cyclic amino groups, which appears to be crucial for NorA inhibition at low concentrations. The presence of the $\mathrm{N}$ benzyl moiety preserves inhibition and contributes to the modulation of the biological effects and ADME properties, depending on its substituents [103].

Another successful example of a hybrid antibacterial/EPI is the fluoroquinolone scaffold accommodating a bis-aryl urea EPI moiety at the C-7 position. This kind of compounds was successful in inhibiting NorA pumps in $S$. aureus. Compound 80, which incorporates a bis-aryl urea motif into the ofloxacin core, proved to be a potent inhibitor of MFS efflux pump systems, achieving $84 \%$ inhibition rate at 10 $\mu \mathrm{M}$. Moreover, these compounds also successfully inhibit
Derivatives of 2-(4-propoxyphenyl)quinolone were synthesized based on the premise that its large hydrophobic area and ability to establish an electrostatic interaction would make them suitable inhibitors of NorA. These derivatives also mimic the quinolone antibacterial core and possess a versatile scaffold, making it prone to be very simply chemically modified. These classes of compounds were obtained by performing modifications in the 2-phenyl- $4 \mathrm{H}$ chromen-4-one moiety, which is a common feature of flavone and flavolignane EPIs [106].

Studies showed that the $O$-substituted 2-phenyl-4hydroxyquinoline derivatives displayed the best activity as NorA inhibitors. Compounds 84 and 85 showed synergistic activity against a strain of $S$. aureus overexpressing NorA 
efflux pumps comparable to reserpine (30) and, comparing the results obtained with a mutant strain, absent of NorA, it was found that these compounds are able to completely restore the antibacterial activity of ciprofloxacin in the resistant strain $[18,106]$. Preliminary SAR studies for this new class of EPIs showed that the best activity is when the quinolone core is replaced with the 2-phenyl-4hydroxyquinoline group with an alkylation in the C-4 hydroxyl group. 2-Ethylamino chains inserted at the $\mathrm{N}-1$ position of the quinolone nucleus or at the C-4 hydroxyl of the quinoline moiety provided compounds with better activity than those carrying the same chain at the C-2 of the phenyl ring. The best substituent for the C-2 phenyl ring appears to be the C-4' propoxy group (Figure 9) [106].

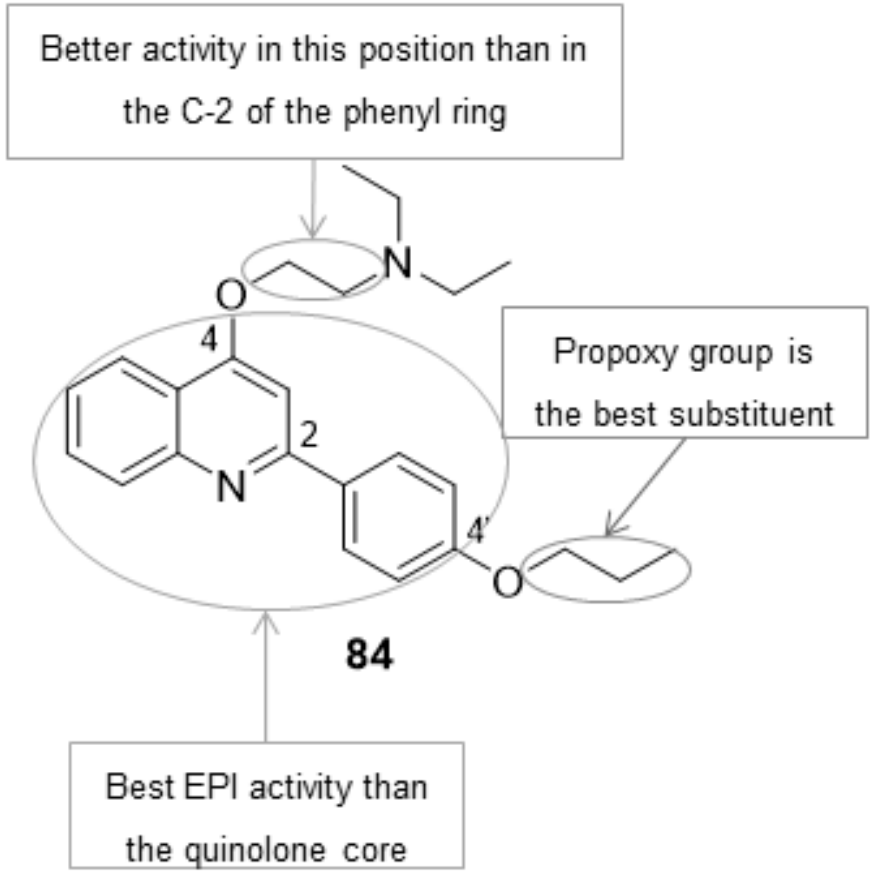

reduction in this strain for ciprofloxacin's MIC. SAR studies for this emerging class show that the best activities come from the compounds bearing the 2-ethylaminoalkyl chains linked to the C-4 hydroxyl group, such as compound 87 . The inclusion of an aliphatic ring including the side chain nitrogen atom led to retention of activity, whereas inclusion in an aromatic ring led to loss of activity, where the nitrogen nucleophilicity was reduced. The mono-substituted compounds, like $\mathbf{8 8}$, with a benzyl group were well tolerated, and showed higher ethidium bromide inhibitory activity than compounds with two benzyl groups as substituents (Figure 9) [107].

3-(Dihydronaphtyl)-propenoic acid amines were evaluated for their EPI activity. Five compounds were synthesized, and

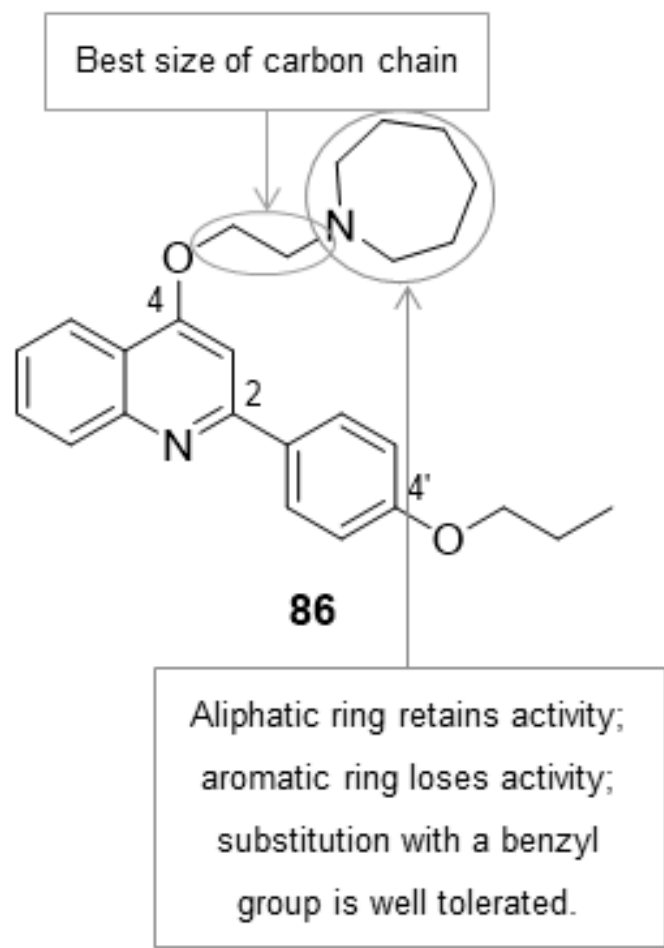

Figure 9 Conclusions from SAR studies for 2-phenyl-4-hydroxyquinoline derivatives 84 and 86.

Taking these results into account, efforts have been placed to obtain more potent NorA inhibitors, introducing $O$-alkyl or different $O$-alkylamino chains at the $\mathrm{C}-4$ position. The strategy was to determine the essential 3D structural requirements for the inhibition of NorA, based on the NorA EPIs described in literature through the use of computational methods, since the 3D structure of this pump was not elucidated yet. The results led to the building of a possible pharmacophore, which was used to design novel compounds, with different substituents at the C-4 position, which were synthesized and biologically evaluated [107]. The compounds were assayed for their ability to reduce the MIC of ciprofloxacin in $S$. aureus strains, and all compounds displayed this decrease for the strain overexpressing NorA pumps. One compound in particular, 86, showed a 16-fold two of them showed good results in inhibiting ciprofloxacin efflux in strains of wild-type and NorA overexpressing $S$. aureus. Further studies were performed, given the low EPI activity of this kind of compounds. Fifteen different compounds were synthesized, taking $\alpha$-tetralone (12) as starting material. Out of these, four compounds showed significant MIC reductions for ciprofloxacin for the NorA overexpressing strain. One compound, 89, even displayed a 16-fold reduction, while other remained in the range of 4- to 8-fold. These studies allowed SAR to be established. Addition of methoxy or allyloxy substituents at the 6,7position of the 3,4-dihydronaphtalene leads to lower or no activity, whereas no substitution originated better EPIs. Saturation of the double bonds lowered the potentiating 
activity (about 2-fold), while unsaturated derivatives made much more potent compounds [108].

Boronic species have been described for a variety of activities, namely antimicrobial and antineoplasic. Organoboron compounds, oxazaborolidines, boronic esters, and boronic acids are of particular interest, within this class. Fontaine et al. (2014) developed studies on these molecules, starting from a screening of a library of approximately 150 compounds, out of which 24 were hit compounds (exemplified with compounds 90 and 91), and were able to restore the activity of ciprofloxacin by 4-fold at concentrations of 0.5 to $8 \mu \mathrm{g} / \mathrm{ml}$ against the NorA overexpressing strain of $S$. aureus [109]. Preliminary SAR studies demonstrated the need of the boron atom for activity. Most pyridine-3-boronic acids showed best potentiating activity (e.g., compound 90), whereas pyridine-4-boronic derivatives showed no activity, with exceptions, including a fluorinated ester. The shift of the boronic moiety from C-3 to C-4 led to a loss of activity and the trigonal boronic acid function gave better results that the sodium boronate salts and the potassium trifluoroborate. Concerning the pyridine3 -boronic acids, the most promising compounds appear to be the 6- and the 5,6-disubstituted ones, being the latter compounds less potent. Cyclisation at the C-5 and C-6 positions was well tolerated, as was the introduction of a methyl at the C-5 position. For the benzene analogues, which displayed good activity, cyclisation at the C-3 or C-4 was also well tolerated, as was the introduction of a methyl. A substituent at the para position to the boronic moiety proved to be very important. Two compounds were particularly efficient in inhibiting NorA, and did not display intrinsic antibacterial activity and cytotoxicity: the 6benzyloxypridine-3-boronic acid 90, which displayed the highest activity, potentiating ciprofloxacin by 4-fold at a concentration of $16 \mu \mathrm{g} / \mathrm{ml}$, and the 4-benzylxybenzene boronic acid 91 [109].

Compound 90, being the most promising compound, was considered a hit, and its structure was used as a model for the synthesis of new derivatives. Therefore, the 6-benzyloxy group was substituted with (aryl)alkoxy chains with variable lengths (compounds 92 and $\mathbf{9 3}$ as examples), in an attempt to explore the putative hydrophobic binding site of NorA, and substituents were added to the phenyl ring. The ether linkage between the pyridine ring and the 6-benzyloxy group was substituted as well. The first substitution made was the replacement of the 6-benzyloxy group with alkoxy chains of diverse lengths, as to explore the size of the hydrophobic pocket of NorA. The potency of the compounds increased with the increase of the size of the side chain, although the compound with a 11-carbon side chain displayed no activity whatsoever. A new series of compounds with a phenyl ring at the end of the side chain were synthesized, with the aim of enhancing hydrophobicity, and the results showed these compounds were more efficient. It was concluded that the compound with the best performance possessed a side chain with seven carbon atoms and a phenyl group: compounds $\mathbf{9 2}$, 6-(3-phenylpropoxy)pyridine-3-boronic acid, and 93, 6-(4phenylbutoxy)pyridine-3-boronic acid, showed the highest activity in the potentiation of ciprofloxacin (4-fold). The introduction of substituents in the phenyl ring of compound 90 led to unsatisfactory results, with the best compound displaying moderate antibacterial activity. A methyl group in the C-5 position did not show influence on the activity, and the modification of the ether linkage led to varied effects: introduction of a sulfanyl, thus conserving the H-bonding acceptor capacity, led to similar potentiating activity, whereas the introduction of an amine linkage, an H-bond donor, led to a decrease in the activity. Finally, the shift of the benzyloxy group from the C-6 to the C-5 resulted in no activity [110].

Compounds AE-848/42434549 (94) and AN-465/42885978 (95) were hits in a virtual screening of novel NorA efflux pump inhibitors in $S$. aureus. The structures deemed as active in silico were later synthesized and evaluated on their ability to prevent ethidium bromide efflux, as well as ciprofloxacin potentiation. Compound 94 and 95 were as potent as reserpine (30) for ethidium bromide efflux and more potent at synergizing with ciprofloxacin. These hit compounds 94 and 95 paved way for the synthesis of new analogues, which allowed SAR studies to be made, in order to assess which parts of their structure were determinant for the inhibition of NorA. For compound 94, it was shown that limited modifications to the sulfone and amide group do not cause significant changes in activity. On the other hand, modifications to the imidazole ring result in a reduction of activity. Replacement of the 1-methylpyrrole ring with a furan also shows a decrease in the activity of the compound. As for compound 95, the $O$-benzyl group is essential and the introduction of a benzyl in the secondary nitrogen leads to a complete loss of activity. Furthermore, the methyl group of the 2-(1-phenyl-1-propanol) moiety can be replaced with a 2(1-phenyl-1-ethanol) portion without a decrease in the activity [111].

\subsubsection{Existing Drugs}

Several drugs already available for the treatment of various diseases have shown potential to be used as MFS EPIs. Reserpine (30), mentioned before, is one of them. Other drugs include verapamil, omeprazole, paroxetine and chlorpromazine. Even though bacterial MFS transporters and mammalian $\mathrm{ABC}$ efflux systems, as P-gp, lack structural homology, they share similar substrate profiles, which means that mammalian MDR inhibitors can also present bacterial efflux inhibition. However, the concentrations needed for efflux pump inhibition are too high, which means that toxic effects can arise if these compounds are used for this purpose [18], [112]. In fact, the addition of verapamil to chemotherapy was accompanied with cardiac arrhythmia and hypotension [113]. This leads to the requirement of developing analogues of these existing drugs maintaining the desired EPI activity at concentrations that do not display toxicity [112].

The antiarrhythmic verapamil, a known P-gp inhibitor, has shown moderate NorA inhibitory activity in both $B$. subtilis and $S$. aureus, enhancing fluoroquinolone activity [114-115]. 
However, the best results were obtained with proton pump inhibitors omeprazole (96) and lansoprazole. Therefore, a series of pyrrolo[1,2-a]quinoxaline derivatives mimicking the omeprazole structure (96) were designed, with the aim of evaluating their EPI activity against NorA. All the eleven derivatives showed a reduction in norfloxacin's MIC, being compound 97 the most active, reducing the MIC by 16-fold at $128 \mu \mathrm{g} / \mathrm{ml}$. These compounds did not show disturbance of the electrical potential and the transmembrane $\mathrm{pH}$, excluding the alteration of the proton-dependent pump as the mechanism of action. Instead, it is thought that these compounds interact directly with NorA. It was shown that the benzimidazole moiety is critical for the activity of these compounds (Figure 10), and replacing it with an imidazole, a pyridine or a pyrrolo[1,2-a]quinoxaline ring resulted in loss of potency. Furthermore, if there is a methoxysubstituent in the benzimidazole ring, potency increases. Introduction of chlorine in the $\mathrm{C}-7$ of the pyrroloquinoxaline heterocycle provided an efficient restoration of norfloxacin's bactericidal activity. Finally, the benzimidazolyl group was also important for activity [112]. suggesting that sulfur, an electron-rich atom, potentiates the EPI activity [116].

GG918 (19) was discovered in a screening program aimed to identify inhibitors of mammalian P-gp, and was able to increase the concentrations of paclitaxel. This compound did not present antibacterial activity against the strains of $S$. aureus tested. However, the co-administration with norfloxacin resulted in a 4-fold reduction in the MIC of the NorA overexpressing strain [52].

Two mammalian MDR inhibitors, biricodar (100) and timcodar (101), confer increased drug sensitivity to cells expressing both the P-gp and multidrug resistance protein (MRP)-1 efflux systems. They also show potentiation of multiple antibiotics in Gram-positive bacteria. In fact, both compounds were able to potentiate the activity of ethidium bromide against $S$. aureus, E. faecalis, and $S$. pneumoniae. For the specific case of $S$. aureus, both compounds were able to partially reverse fluoroquinolone resistance. The fact that the MIC of ethidium bromide was non-saturable for the three bacteria tested suggests that $\mathbf{1 0 0}$ targets multiple efflux pumps [117].

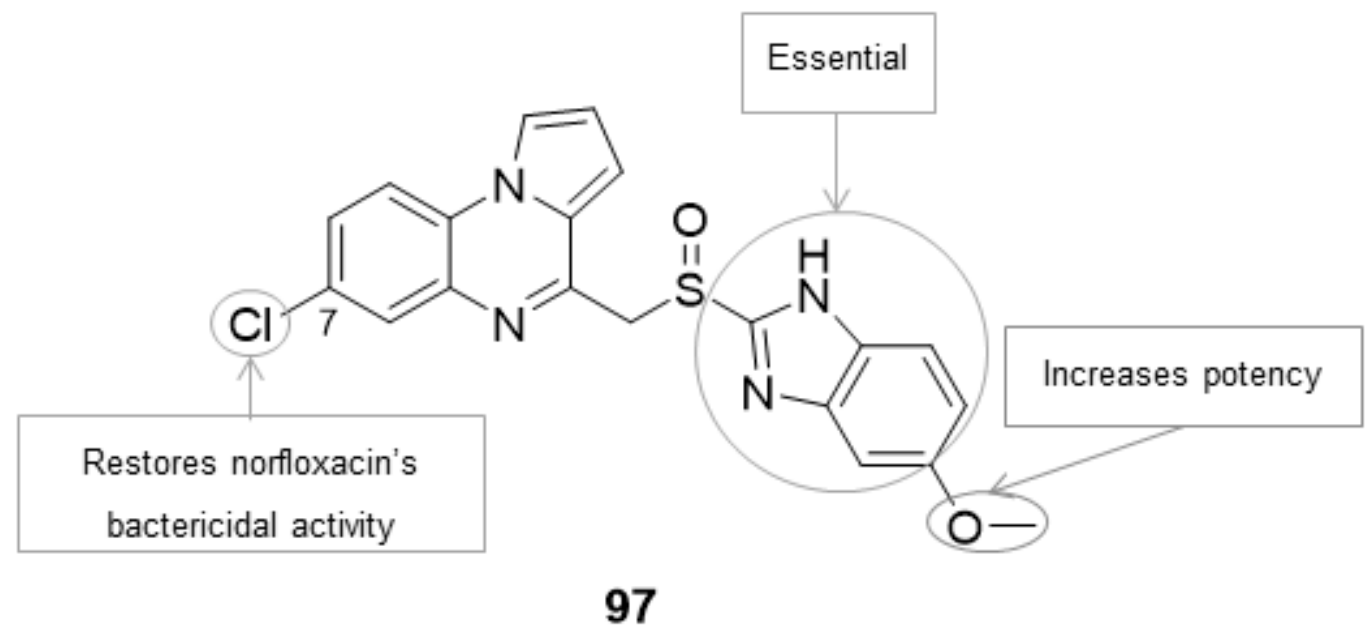

Figure 10 Conclusions from SAR studies for omeprazole derivatives.

This was not the first time that pyrrolo[1,2-a]quinoxaline derivatives were described as EPIs. In fact, a previous study by the same group produced 12 new 4-[116]pyrrolo[1,2a]quinoxalines and one pyrrolo[1,2- $\alpha]$ thieno[3,2-e]pyrazine compound. Two compounds, 98 and 99, were more active than reserpine (30), diminishing norfloxacin's MIC by 16fold in a NorA overexpressing strain of $S$. aureus. This study showed that 4-[116]pyrrolo[1,2-a]quinoxalines and their 7methoxy analogues were more active, in contrast with the chlorine- and 8-phenyl-substituted compounds, that presented the lowest effects. Replacement of the $\mathrm{N}, \mathrm{N}$ diethylamino group by pyrrolidine, its isomer with restricted conformation, led to enhanced EPI activity, whereas replacing it with a piperidine ring led to more flexible and, consequently, less active compounds. The pyrrolothienopyrazine 99 moiety yielded the best results,
A series of polysubstituted pyrroles was screened for their Pgp inhibition activity, which made way for the identification of a lead, and the compounds were further investigated for their bacterial efflux pump inhibition activity. A simplified pyrrole alkaloid, 102, displayed dual activity as both a P-gp and a NorA inhibitor against $S$. aureus. This compound led to 4-fold improvement in the MIC of ethidium bromide in the NorA overexpressing strain. Compound $\mathbf{1 0 2}$ was also able to reduce ciprofloxacin's MIC by 8 -fold when at $50 \mu \mathrm{M}$ [118].

Tariquidar (103), a third generation MDR modulator, with activity in inhibiting P-gp and BCRP efflux systems, has shown promise in inhibiting efflux pumps in $S$. aureus. In fact, this compound showed an increase of the isotopic form of ciprofloxacin in all investigated strains, displaying best results in the strain that overexpresses the NorA pump. [119] 
The class of phenylpiperidine selective serotonin reuptake inhibitors (PSSRI), which includes the antidepressant paroxetine (104), showed efflux-related potential against $S$. aureus, particularly that conferred by NorA. In fact, paroxetine and its isomer, 105, showed potency with respect to inhibition of this efflux pump against ethidium bromide. The isomer 105 also interfered with the accumulation of norfloxacin by NorA, reducing by 4 - to 8 -fold its MIC. [120] Femoxetine (106) is a paroxetine derivative, with activity as an EPI. [120] Studies were performed as to determine which moieties in paroxetine- and femoxetine-like PSSRI were detrimental for efflux pump inhibition in $S$. aureus, and conclusions from SAR are highlighted in Figure 11. Synthesis of derivatives showed that the fluorine atom on the phenylpiperidine moiety is not associated with the activity. The $N$-substitution of the piperidine ring yields different kinds of activity, suggesting that the NH group forms a positive binding contact with the target pump, which does not happen if there is a $N$-substitution (compound 106). The phenyl ether substituents also play an important role as determining the EPI activity. [121]

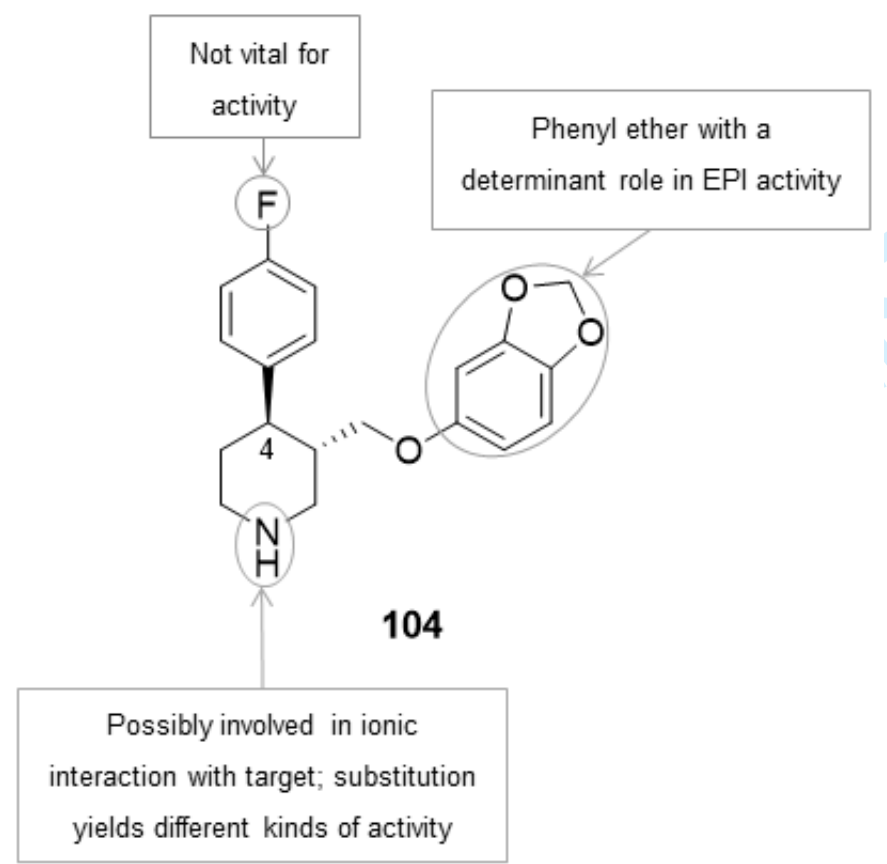

Figure 11 Conclusions from SAR studies for paroxetine derivatives.

Also for this kind of compounds, a study was performed, in order to conclude whether the 4-phenyl ring is required for EPI activity and the effects of changes in the two-atom aryloxymethyl linker, replacing it with a thioether, amine, and alkene linker portions. Compounds 107 and 108 displayed the lowest MIC against $S$. aureus. Synthesis of multiple derivatives proved that the 4-phenyl moiety is not vital for EPI activity and that a variety of two-atom linker groups for the 3-aryl piperidine moiety can maintain EPI activity [122].
The ciclooxigenase-2 (COX-2) inhibitor celecoxib (109) has also received attention for its MDR inhibitory activity. There had been a link between COX-2 and $m d r l$ gene expression, which has consequences in cancer chemo resistance, and the use of celecoxib (109) reversed drug resistance. If this is true for mammalian efflux pumps, for bacteria there is no evidence of a COX-2-like gene, suggesting that celecoxib acts differently in inhibiting MDR in bacteria. A study was carried out in order to prove this hypothesis, which demonstrated that this drug helped in increasing sensitivity to antibiotics in S. aureus and Mycobacterium smegmatis. These effects probably occur due to the blockage of MDR transporters involved in the efflux of antibiotics, such as ampicillin, ciprofloxacin, kanamycin, and chloramphenicol. The observation of the accumulation of ethidium bromide intracellularly leads to the conclusion that this compound acts as a NorA inhibitor [123].

As celecoxib (109) demonstrated such promising activity, analogues were tested, which bore low or none antiinflammatory activity and possessed the 1,4dihydropyrazolo[4,3-c]-benzothiazine-5,5-dioxide nucleus. Ethidium bromide efflux inhibition was tested first in silico, and the 17 molecules that fit the chemical space requirements were then reduced to six, based on the activity prediction. These compounds were then screened for their in vitro NorA inhibitory activity, using an overexpressing strain of $S$. aureus. One derivative in particular, compound 110, showed the best activity concerning ethidium bromide efflux (76.9\%) which is better than celecoxib (109) (64.5\%). When in combination with ciprofloxacin, $\mathbf{1 1 0}$ displayed results comparable to reserpine (30) and better than paroxetine (104), which makes it a lead compound for the development of inhibitors of this kind [124].

Phenothiazine and thioxanthene derivatives are currently used as neuroleptic and antiemetic agents, and have been described as modest, but broad, antibacterial agents. Although their antibacterial activity is not enough to be clinically relevant, they have shown synergy with standard antibiotic agents, and have also been associated with the inhibition of eukaryotic MDR efflux pumps, such as P-gp. The mechanism through which these compounds exert their antimicrobial potentiation is not yet fully understood. However, it is thought that it might have to do with the inhibition of efflux pumps [125]. A study focusing on the activity of several compounds of these families against $S$. aureus was carried out, with the main aim of showing their potential in inhibiting NorA. The compounds tested, chlorpromazine, fluphenazine, thioridazine, prochlorperazine (111), cis(Z)-flupentixol, and $\operatorname{trans}(E)$-flupentixol (112), displayed intrinsic antimicrobial activity and good results when ethidium bromide efflux was assayed against a NorA overexpressing strains of $S$. aureus. Additionally, phenothiazine 111 and thioxanthene $\mathbf{1 1 2}$ were also able to reduce the proton motor force of $S$. aureus, by reducing the transmembrane potential [125].

Since the phenothiazine moiety proved itself to work as a template for the synthesis of new MDR EPIs, it was chosen to be the scaffold for derivatives with improved activity. The 
logic for these studies lied in the elimination of the structural features that were responsible for neuroleptic activity. As such, drastic modifications were made, such as the elimination of one ring of the tricyclic benzothiazine backbone and of the chain linked to the N-10 atom, a tertiary amine whose protonation is crucial for the interaction with the dopaminergic receptor, and the addition of a substituted phenyl ring at the C-3 position, to guarantee better lipophilicity. Therefore, a prototype of new 3-phenyl-2H1,4-benzothiazines was developed. Preliminary screening on the derivatives using a wild-type $S$. aureus strain, both with and without ciprofloxacin, showed variable intrinsic and synergistic activity. Two compounds, 113 and 114, displayed the best activity, while displaying no and weak antibacterial activity, respectively. These compounds were able to inhibit NorA efflux of ciprofloxacin, being suggested that 114 was even able to completely inhibit this pump [126].

Thioridazine (115), a phenothiazine, was also tested for its antibiotic potentiation, either in racemate or in its enantiomeric forms. All the forms were able to reduce the MIC of oxacillin in $S$. aureus and erythromycin in Streptococcus pyogenes, being the L-enantiomer more effective in the last case [127-128].

\section{RND}

RND pumps are found mostly in Gram-negative bacteria, being the most responsible pumps for MDR in this kind of organisms. They present a wide variety of lipophilic and amphiphilic substrates, which include several classes of antibiotics, antiseptics, dyes, and detergents. RND transporters present a unique tripartite complex, constituted by a minimum of twelve transmembrane segments: the transmembrane pump, the outer membrane channel, and the

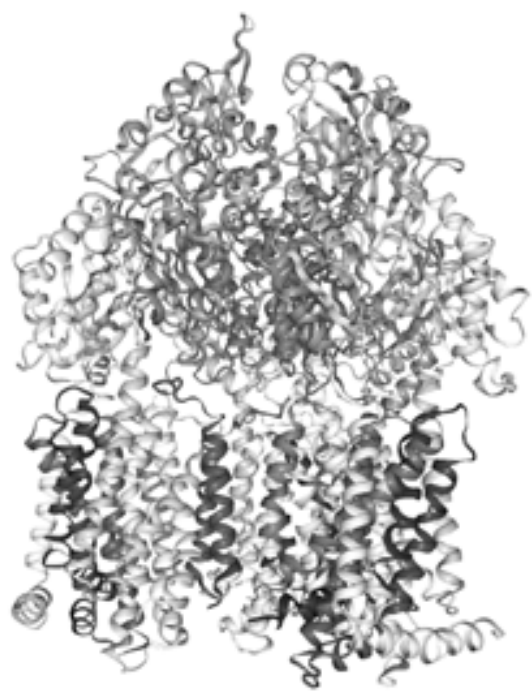

periplasmic adaptor protein, which puts the other two components in contact. This structural organization makes the extrusion of substrates directly into the external medium possible [32, 38]. Even though it has always been thought that RND transporters were exclusive to Gram-negative bacteria, it has recently been identified and characterized FarE in S. aureus, homolog to the AcrB protein in E. coli [129-130].

Within this family, the most studied system is the AcrABTolC, from E. coli. This efflux pump is composed by three essential parts: a resistance-nodulation-division transporter, AcrB; a membrane fusion protein, AcrA; and a multifunctional outer membrane channel, TolC [7, 131-133]. It transports a wide variety of toxic compounds from the intracellular space directly into the medium, evading the periplasm, and using a proton gradient as the energy source [132-133]. Pseudomonas aeruginosa also possesses an efflux system of the RND family, the MexAB-OprM efflux pump, which confers resistance to a wide variety of antibiotics. This was the first and best characterized efflux system in bacteria. It was found that AcrB (Figure 12, PDB 4ZLJ) is homolog to MexB (Figure 12, PDB 2V50), being their structure very similar [7, 134].

Figure 13 comprises all the RND pump inhibitors that will be discussed in the following section.

\subsection{RND inhibitors}

\subsubsection{Natural Products and Derivatives}

One study revealed that the essential oil of Helichrysum italicum can reduce the resistance to chloramphenicol in $E$. aerogenes, $P$. aeruginosa, and A. baumanii. These data suggested that one or more EPIs are present within the essential oil since it was shown that geraniol (116) produced

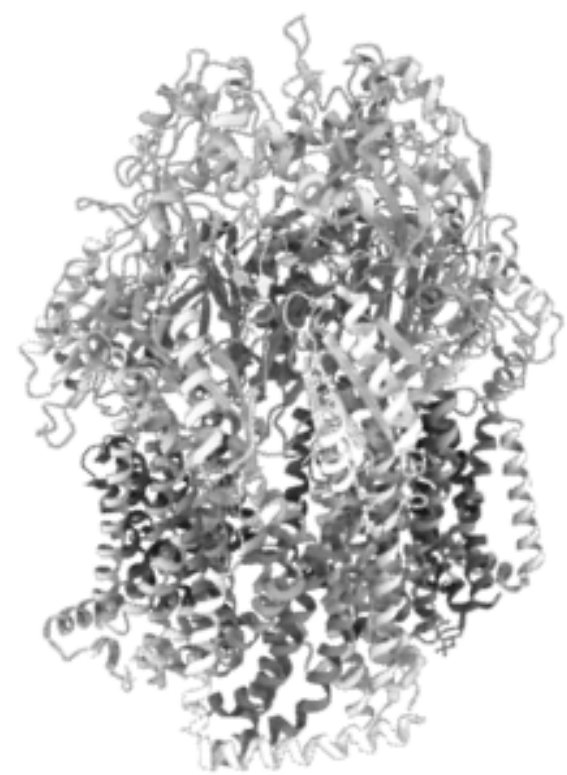

Figure 12 Crystal structures of AcrB (left, PDB 4ZLJ) of E. coli and MexB (right, PDB 2V50) of P. aeruginosa. 


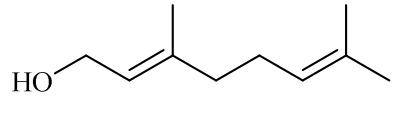

116<smiles>O=c1c(-c2ccc(O)cc2)coc2cc(O)ccc12</smiles>

118

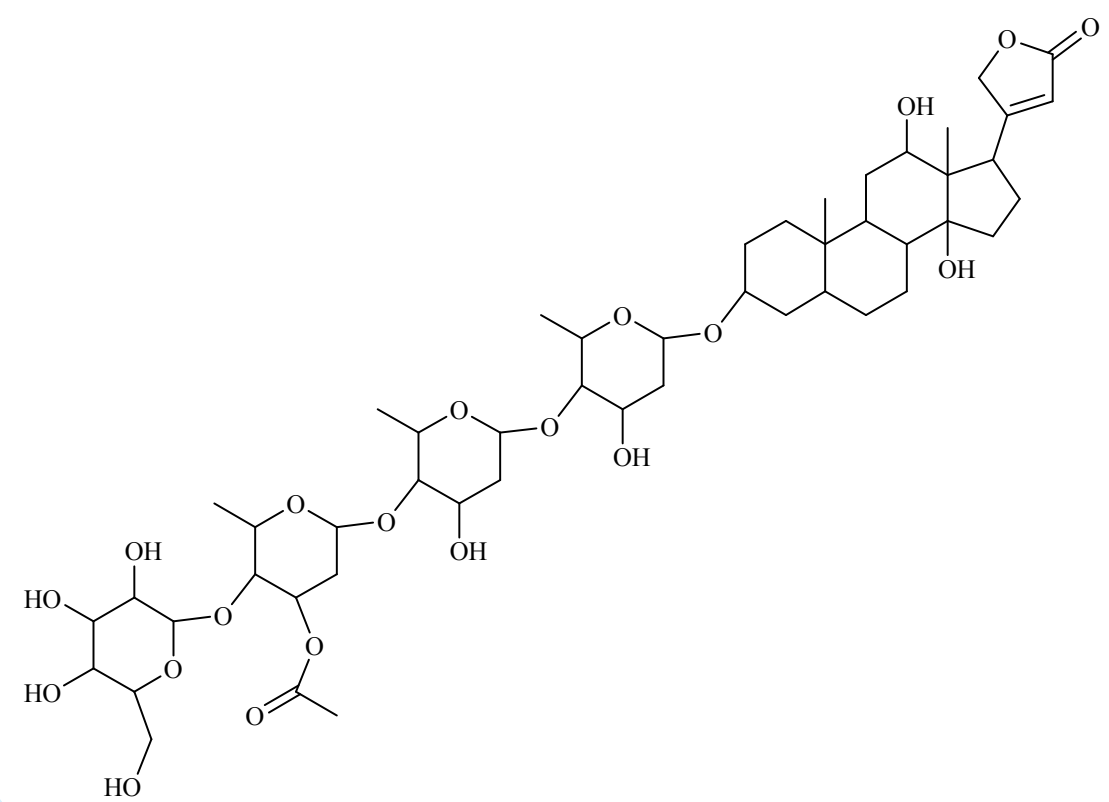

117

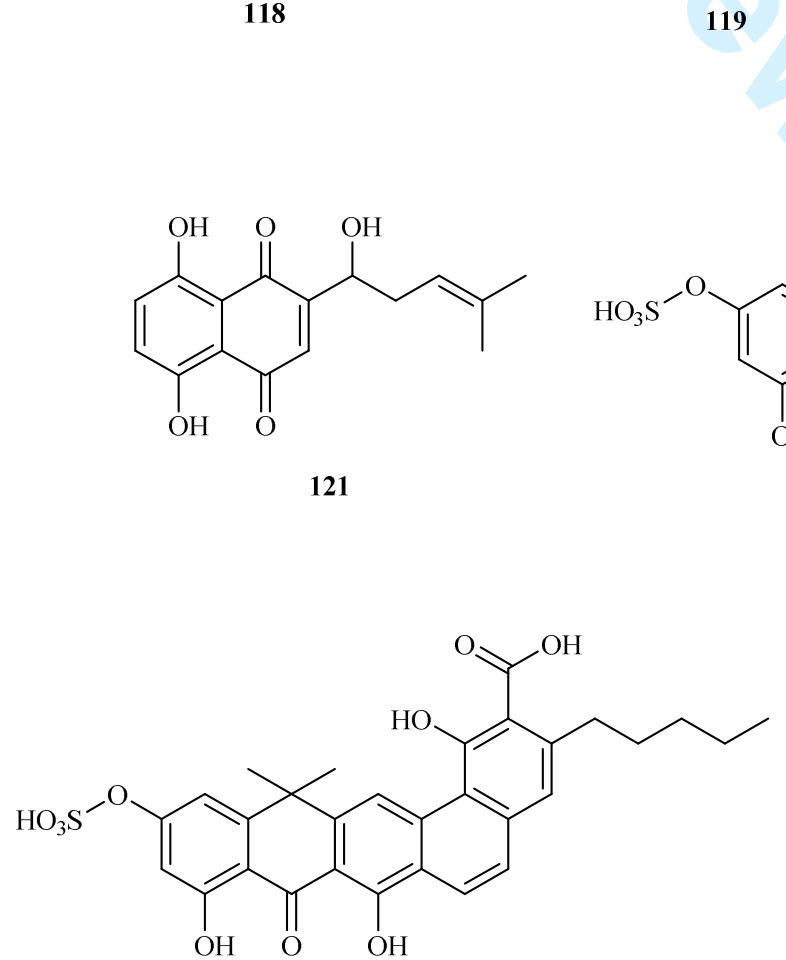

123<smiles>CC1=CC(=O)c2c(O)cccc2C1=O</smiles>

119<smiles>CC(Cc1ccc(O)c(O)c1)C(C)Cc1ccc(O)c(O)c1</smiles>

120

Figure 13 Structures of RND inhibitors $116-154$. Boc = tert-Butyloxycarbonyl. 
Short Running Title of the Article

1

2

3

4

5

6

7

8

9

10

11

12

13

14

15

16

17

18

19

20

21

22

23

24

25

26

27

28

29

30

31

32

33

34

35

36

37

38

39

40

41

42

43

44

45

46

47

48

49

50

51

52

53

54

55

56

57

58

59

60

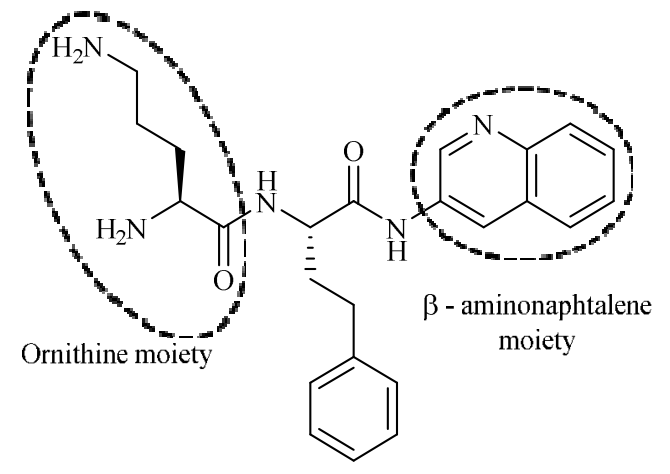

125<smiles>CC1(C)Cc2c(C#N)c(SCCc3ccccc3)nc(N3CCOCC3)c2CO1</smiles>

127<smiles>C=CC(=O)Nc1ccc(CCSc2nc(N3CC(C)OC(C)C3)c3c(c2C#N)CC(C)(C)OC3)cc1</smiles>

129<smiles>FC(F)(F)c1cccc(CN2CCNCC2)c1</smiles>

135<smiles>NC[C@@H]1CN[C@H](C(=O)N[C@@H](CCc2ccccc2)C(=O)Nc2ccc3ncccc3c2)C1</smiles>

126<smiles>CC(=O)Nc1ccc(CCSc2nc(N3CC(C)OC(C)C3)c3c(c2C#N)CC(C)(C)OC3)cc1</smiles>

128

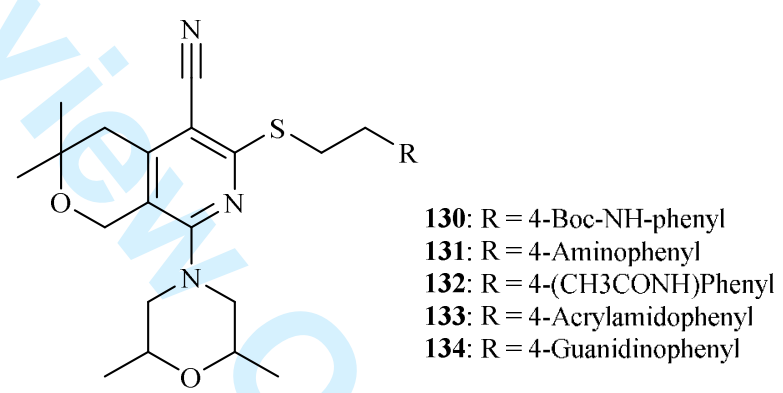

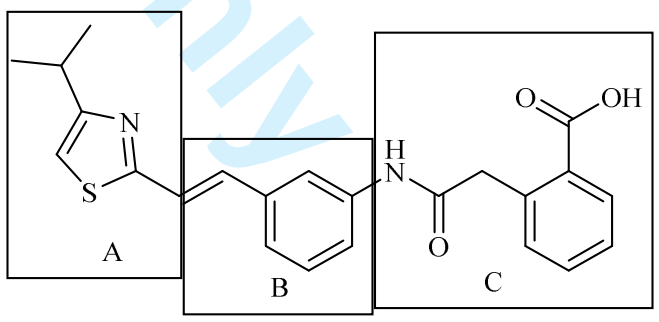

137

Figure 13 (cont.) 
<smiles>CC(C)c1csc(COc2ccn3c(=O)c(-c4nnn[nH]4)cnc3c2)n1</smiles>

138<smiles>CC(C)(C)c1csc(NC(=O)c2ccn3c(=O)c(/C=C/c4nnn[nH]4)c(N4CCC[C@@H](OC(=O)NCC[N+](C)(C)CC(=O)[O-])C4)nc3c2)n1</smiles>

140<smiles>Cc1cc(OCCN2CCCC2)c2cccc(C)c2n1</smiles>

142<smiles>CC(C)c1csc(COc2ccn3c(=O)c(/C=C/C(=O)O)c(-c4ccc(CN(C)C5CC5)cc4)nc3c2)n1</smiles>

139

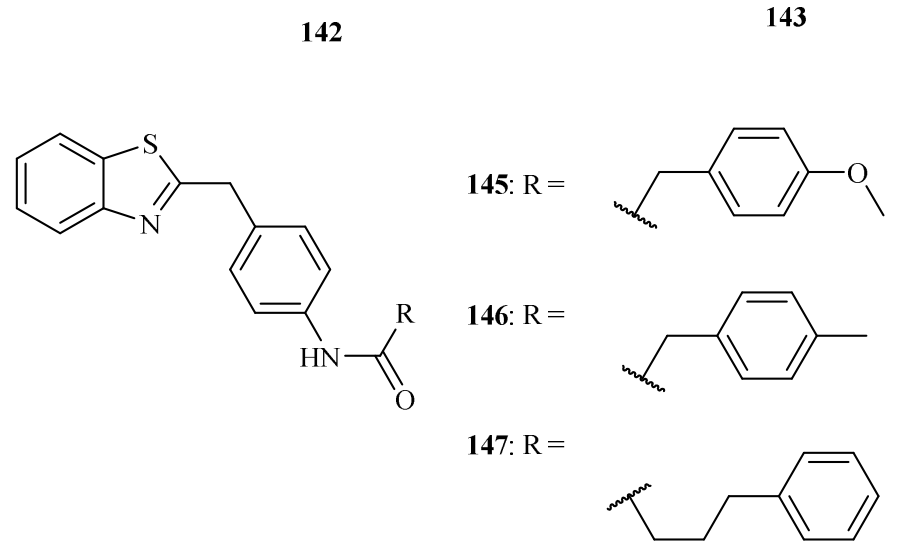<smiles>CC(C)N(CCNc1ccnc2cc(Cl)ccc12)C(C)C</smiles>

143<smiles>c1ccc2c(NCCCN3CCOCC3)ncnc2c1</smiles>

144

Figure 13 (cont.) 
<smiles>CN(C)CCCN1c2ccccc2Sc2ccc(Cl)cc21</smiles>

150<smiles>CN[C@H]1CC[C@H](c2ccc(Cl)c(Cl)c2)c2ccccc21</smiles>

151<smiles>COc1cc(Cc2cnc(N)nc2N)cc(OC)c1OC</smiles>

152<smiles>CNC[C@H](O)c1ccc(O)c(O)c1</smiles>

153

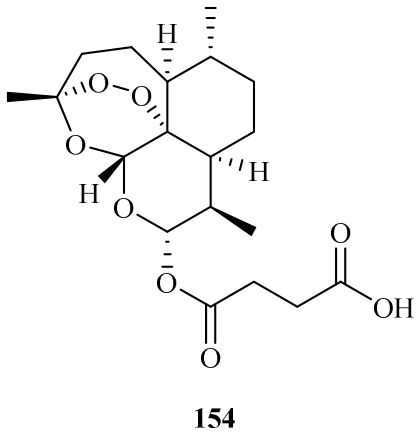

Figure 13 (cont.)

significant restoration of sensitivity to chloramphenicol by 16-fold, and rendered a total sensitivity of the organism when combined with of phenyl-arginine $\beta$-naphtylamide $(\mathrm{PA} \beta \mathrm{N})$, which will be discussed further ahead [135].

A study published by Aparna et al. (2014) had the aim of identifying natural compounds from plants that present efficacy in potentiating effects of antibiotics in $P$. aeruginosa overexpressing MexAB-OprM and E. coli overexpressing AcrAB-TolC through in silico virtual screening and pharmacophore approaches [136]. The compounds with the best in silico results were then tested for their EPI activity. The two compounds that showed the best activity in potentiating the activity of carbenicillin and levofloxacin in P. aeruginosa and E. coli were lanatoside C (117) and daidzein (118). Lanatoside $C$ (117) is cardiac glycoside that inhibits the $\mathrm{Na}^{+}-\mathrm{K}^{+}$-ATPase, which can also be the explanation for its EPI activity against MexB and AcrB. Daidzein (118) is an isoflavone that has previously showed slight EPI activity in Mycobacterium smegmatis and as a modulator of P-glycoprotein, in human cervical carcinoma KB-V1 cells [136].

An in silico study by Ohene-Agyei et al. (2014) identified five phytochemicals that could be docked similarly to PA $\beta N$ in the binding pocket of AcrB. Of these five, three of them were able to decrease resistance to antibiotics by inhibiting the AcrAB-TolC system: plumbagin (119) increased sensitivity to erythromycin, chloramphenicol, and tetraphenylphosphonium; nordihydroguaretic acid (NDGA) (120) potentiated the activity of erythromycin, chloramphenicol, tetraphenylphosphonium, novobiocin, and tetracycline; and shikonin (121) decreased resistance to tetraphenylphosphonium [137].

A study proved the usefulness of curcumin (64) as a RND EPI at a concentration of $50 \mu \mathrm{g} / \mathrm{ml}$, increasing the sensitivity of carbenicillin, ceftazidime, and meropenem in $P$. aeruginosa, in decreasing order. It also managed to circumvent the resistance to gentamicin and ciprofloxacin in resistant isolates, which $\mathrm{PA} \beta \mathrm{N}$ failed, leading to the assumption that curcumin inhibits efflux pumps differently from PA $\beta \mathrm{N}$ [138].

A screening of 85000 microbial fermentation extracts derived from 3600 strains of actinomycetes and 3500 strains of fungi was made, with the aim of discovering EPIs that increased sensitivity of levofloxacin in $P$. aeruginosa overexpressing the MexAB-OprM or the MexEF-OprN pump. Two compounds, EA-371 $\alpha$ (122) and EA-371- $\delta$ (123), produced by a strain of Streptomyces sp., demonstrated inhibitory activity against MexAB-OprM [139].

\subsubsection{Synthetic Compounds}

The first compound with potent inhibition of RND efflux pumps was PAßN (124), a dipeptide amine. This dipeptide was identified as a hit compound from the screening of 200 000 samples of small molecules that potentiate the activity of antibacterial levofloxacin against strains of $P$. aeruginosa that overexpressed MexAB, MexCD and MexEF pumps [28, 140]. Compound $\mathbf{1 2 4}$ is a peptidomimetic, developed for using in adjunctive therapy. This compound is a substrate for RND pumps, acting as a competitive inhibitor of multidrug efflux systems, such as MexB, MexD, and MexF, binding to the substrate pocket, impeding antibiotic binding and/or extrusion. Particularly, this EPI binds in a location close to the antibiotic binding site, generating steric hindrance, making it more difficult for the antibiotic to bind. In other words, less quantity of antibiotic is exported, making its intracellular levels higher, thus restoring antibiotic sensitivity [11, 25, 27-28, 141-143]. However, 124 can affect the integrity of the membrane when used in high 


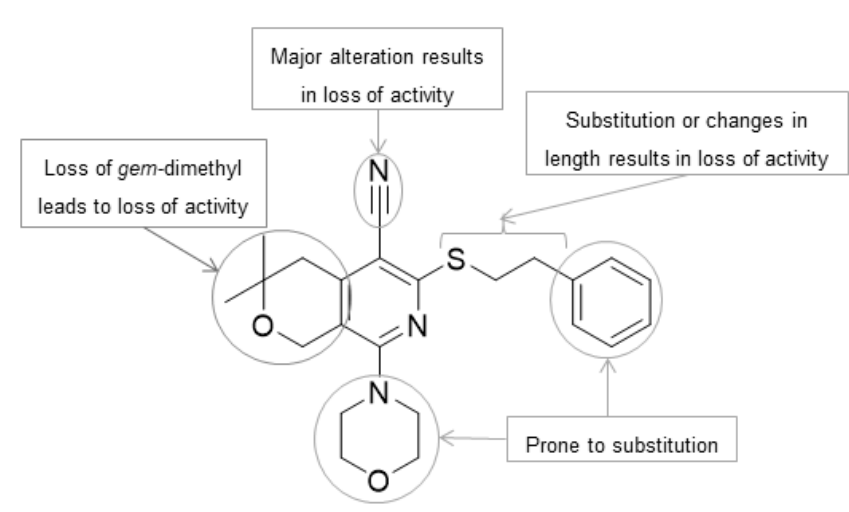

Figure 14 Conclusions from SAR studies for MBX2319 (127) (adapted from [28]).

concentrations, making it prone to the emergency of resistant profiles, namely the modification of the lipopolysaccharide structure, leading to changes in drug penetration [24].

$\mathrm{PA} \beta \mathrm{N}$ (124) has been validated against the AcrAB-TolC in Klebsiella pneumonia, E. coli, Salmonella enterica serovar Typhimurium and Enterobacter aerogenes, and in multiple homologous systems including Acinetobacter baumanii, Campylobacter jejuni, and Campylobacter coli $[11,142]$. Its potency has been demonstrated against the norfloxacin resistance conferred by the Mex efflux system of Pseudomonas aeruginosa, the AcrAB efflux systems of the Enterobacteriaceae, and the erythromycin efflux system of $C$. jejuni [19].

Studies of SAR demonstrated that the basic middle amino acid, arginine, was detrimental for activity, although a substitution with L-lysine showed the same potency as the lead, and the introduction of L-ornithine, originating compound L-Phe-L-Orn- $\beta$-Na, showed a 2 -fold increased potency. As the ornithine moiety provided a simpler synthesis, it became the standard middle residue in successive compounds [25, 142, 144]. However, these compounds were not stable upon incubation with human serum, due to the occurrence of cleavage of peptide linkage between both amino acids, which is explained by the fact that natural (L) amino acids are contained within its structure. In order to overcome this problem, methylation of the NH that links both amino acids was achieved, maintaining potency and being this derivative able to resist serum proteases. Serum stability was also increased by replacing the L-amino acid with D-amino acid [25, 142]. In the same study, the authors also proved that the amino acid in the aal position (Figure 13, 124) should be appropriately substituted, and the replacement of phenylalanine with a homophenylalanine led to improved potency. The problem with this compound was the fact that the ornithine moiety could easily form a lactam, rendering the compound inactive. This was overcome by switching the L-ornithine with the Lphenylalanine, which originated L-Orn-L-hPhe- $\beta-\mathrm{Na}$, which originated a compound just as potent, but with no propensity to form a lactam. As for the $\beta$-aminonaphtalene moiety, it was proven that replacing it with 3 -aminoquinoline led to reduced cytotoxicity and intrinsic antibacterial activity, even though its potency was slightly reduced [25, 142]. These efforts originated the compound L-Orn-L-hPhe-3-NHQ (125). In conclusion, the analogues synthesized by Renau et al. (1999) did not show improvements in potency when compared to $\mathrm{PA} \beta \mathrm{N}$ (124), but showed increased stability [142].

The same group developed further studies with this type of molecules and demonstrated that conformational restriction of the amino group of ornithine showed less toxicity, while maintaining potency. Such compounds were attained by adding a proline substituent, thus incorporating the two basic residues necessary for activity, which resulted in the successful synthesis of constrained derivatives, more potent and less toxic than 125. The most interesting derivative, compound $\mathbf{1 2 6}$, was as potent as $\mathbf{1 2 5}$, but more than 4 -fold less toxic. This compound also retained the activity against RND efflux pumps MexAB-OprM, MexCD-OprJ and MexEF-OprN. Although this compound showed similar protein binding in rats as $\mathbf{1 2 5}$, its pharmacokinetic parameters were improved, and its efficacy in combination with levofloxacin was demonstrated in an in vivo model [145].

In a latter study developed by the same group, several nonpeptide analogues were synthesized, and it was hypothesized that the peptide backbone is not essential for the activity of this type of compounds. The results suggested that the inhibition of pumps was possible due to the di-cationic nature of the compound, the appropriate lipophilicity and a disposition similar to that of compound 125 [146].

MBX2319 (127) is a pyranopyrimidine EPI inhibitor of AcrAB-TolC efflux pump in E.coli and other Enterobacteriaceae [141]. Compound $\mathbf{1 2 7}$ did not show any antibacterial activity, which is a positive feature for EPIs. It also potentiated the antibacterial activity of fluoroquinolones, such as ciprofloxacin and levofloxacin, and $\beta$-lactams, such as piperacillin against strains of $E$. coli that presented AcrAB-TolC efflux pumps [28, 141]. In $P$. aeruginosa, although compound $\mathbf{1 2 7}$ presents activity against RND-type pumps, it is unable to penetrate the outer membrane, which is highly selective [28].

In $E$. coli, the target of compound $\mathbf{1 2 7}$ is the membrane transporter AcrB. This molecule binds to the hydrophobic trap of the $\mathrm{T}$ protomer, interacting with the hydrophobic residues that constitute the deep binding pocket as a hydrophobic trap. $\pi-\pi$ Interactions also occurs, between the pyridine ring of 127 and the aromatic side chain of Phe-628. The phenyl and morpholinyl groups also interact with Phe178 and Phe-615. The dimethylenesulfide linker and the geminal dimethyl group present van der Waals interactions between the side chain of Phe-178, and Tyr-327 and Met573, respectively [28, 147].

SAR studies demonstrated that the maintenance of activity depended on the geminal dimethyl moiety of the tetrahydropyran, the nitrile group and the length of the dimethylenesulfide linker. This data is presented in Figure 14. On the other hand, the morpholine moiety and aryl group are both prone to substitution, originating more stable 
derivatives in terms of activity, solubility, and metabolism [28]. Studies with this scaffold have shown that non-acidic substituents can be added to the phenyl group, in order to improve potency and CYP450 inhibition, and modifications to the morpholinyl group has greater effects on solubility and stability [140].

Analogues were synthesized by Sjuts et al. (2016), by changing the structure and functional groups of compound 127 (148). They reached the conclusions that the introduction of a 2,6-dimethyl to the morpholinyl group improved both microsomal stability and EPI activity. The replacement of the morpholinyl group with 2methoxyethylpiperazinyl improved aqueous solubility, at the cost of a slight reduction of EPI activity. Two analogues, 128 and 129, resultant of the combination of 2,6dimethylmorpholinyl and acetamide or acrylamide, respectively, on the phenyl group, resulted in a 10-fold to 20fold increase in EPI activity against E. coli, respectively, comparing to MBX2319 (127) [28, 148]. The increased EPI activity of these compounds has its basis in the acetamide and acrylamide groups and the morpholinyl group. The acetamide groups are engaged in highly ordered and complex hydrogen bonds, with its center in a solvent water molecule, which plays the role of a hydrogen bond donor to the carbonyl backbone oxygen of Ala-286 and Gln-151 side chain of AcrB. The acrylamide group forms a bridging hydrogen bond through a water molecule to the side chain of Gln-176 [28].

Nguyen et al. (2015) also synthesized a series of analogues, with the morpholine scaffold substituted by a 2,6dimethylmorpholinyl group (140). Many of these analogues exhibited a solubility improvement of 10-fold, compared to compound 127. The most potent and promising compounds of these series were compounds 130, 131, 132, 133 and 134 [140].

Through a screening of an $N$-heterocyclic organic compound library, Bohnert and Kern (2005) were able to find a novel class of EPIs, the arylpiperazines [25, 149]. The aim of their work was to find compounds that would potentiate levofloxacin potency against strains of $E$. coli overexpressing $a c r A B$ and acrEF. This was fulfilled when they found phenylpiperazine derivatives with promising activity [25, 149]. SAR studies suggested that elongation of the spacer between the benzene ring and the piperazine ring would enhance potency. Also, substitutions on the benzene ring by halogen atoms led to an increase in potency [24, 149]. On the other hand, the potency would decrease when an ethyl or phenyl group was added to the piperazine ring. With this, the authors found the most potent phenylpiperazine, meta-TFMPP (135), and naphthylpiperazine, 1-(1-naphthylmethyl)-piperazine (NMP) (136) [149].

NMP (136) was the most potent unsubstituted arylpiperazine, able to increase the intracellular concentration of chloramphenicol, tetracycline, linezolid, fluoroquinolones, and macrolides. Although it does not display action in $P$. aeruginosa, it shows effectiveness in $A$. baumanii and several Enterobacteriaceae [11, 23]. EPI activity was also reported in clinical isolates of $E$. coli, most relevantly in fluoroquinolone resistance [150]. Through computational analysis, it was found that NMP (136) binds with high affinity to the lower part of the deep pocket of the $\mathrm{B}$ monomer, overlapping in part the binding site of chloramphenicol [11]. The binding site for NMP (136) includes interactions with hydrophobic residues near the hydrophobic patch and Gly-617 of the G-loop. This data suggests that NMP (136) interferes with the movement of the G-loop, important for extruding substrates, thus inhibiting the action of AcrB [25].

Nakayama et al. (2003) performed a series of syntheses in order to attain a compound specific for the inhibition of a MexAB-OprM specific EPI [151-152]. They identified a hit, compound 137, through high-throughput screening for levofloxacin potentiation using a strain of $P$. aeruginosa strain overexpressing MexAB-OprM. The features this group was looking for was the effective inhibition of efflux pump and the lack of intrinsic antibacterial activity, since efflux pumps are not vital for the organism's survival [151-152]. It was shown that this compound effectively potentiated the activity of levofloxacin, leading to an 8-fold decrease in the antibiotic's MIC. However, its physicochemical properties were problematic: water solubility was poor and it presented high affinity to serum albumin. Therefore, they divided the molecule into three parts, A, B, and C (137) and looked for alternative scaffolds and substituted polar groups, in order to reduce serum protein binding [152].

It was shown that when a carboxamide was introduced in $\mathrm{A}$, the affinity to serum albumin was diminished, as was its activity. More polar moieties led to a complete loss of activity. The strategy for the styrene portion (B) that was the most successful was to substitute the benzene ring to a 3,5disubstituted pyridine, which had both good activity and no major serum influence. As for the vinyl group in B, it was demonstrated that molecules bearing methyloxy and ethylene moieties displayed favorable activity profiles. As for part C, the basis of the modifications was the fact that the introduction of bulky or hydrophilic groups adjacent to the carboxyl group would reduce protein binding. An enantiomeric pair containing a dioxolane moiety presented both activity and serum stability, being the $S$ enantiomer more active [152].

Regarding the general scaffold, new compounds were designed, keeping in mind that the thiazole moiety was crucial for activity. Therefore, a molecule having a thiazole side chain attached to the $\mathrm{C}-7$ position of a quinolone was synthesized. Substitution with a tetrazole portion yielded better results than substitution with a carboxylic acid. One compound (138) presented the best activity and serum stability [153].

Further efforts were placed in order to increase the potency of this compound. The in vitro activity was enhanced by the introduction of a hydrophobic group at the 2-position of the pyridopyrimidine scaffold, and hydrophilic substitution would not compromise the activity. The most promising compound had the inclusion of an olefin spacer between the tetrazole and the pyridopyrimidine scaffold, with the 
limitation of being prone to photoisomerization [154]. To circumvent this, the ethylene tether between the pyridopyrimidine was replaced with an amide bond, providing stability against photoisomerization and, unexpectedly, improving potency. However, another problem arose: the lack of solubility for intravenous use [155]. The following step was the introduction of a secondary amine, giving a zwitterionic analogue, with retention of activity. C-2 Analogues, namely with cyclic substituents, also showed promising results [156]. Once again, the problem of solubility arose. Therefore, efforts were placed in making the molecule more hydrophilic, with the introduction of substituted aromatic moieties. Analogues 4-substituted were the most potent, and the introduction of a morpholine moiety led to increased solubility, maintaining the serum stability. Compound $\mathbf{1 3 9}$ was the most balanced molecule in terms of the desired features [157]. The last refinement made was the introduction of a quaternary ammonium salt, which led to D13-9001 (140). Adding to its high solubility and safety, it was able to potentiate the activity of aztreonam [158]. Figure 15 illustrates the SAR established for 140 and derivatives.

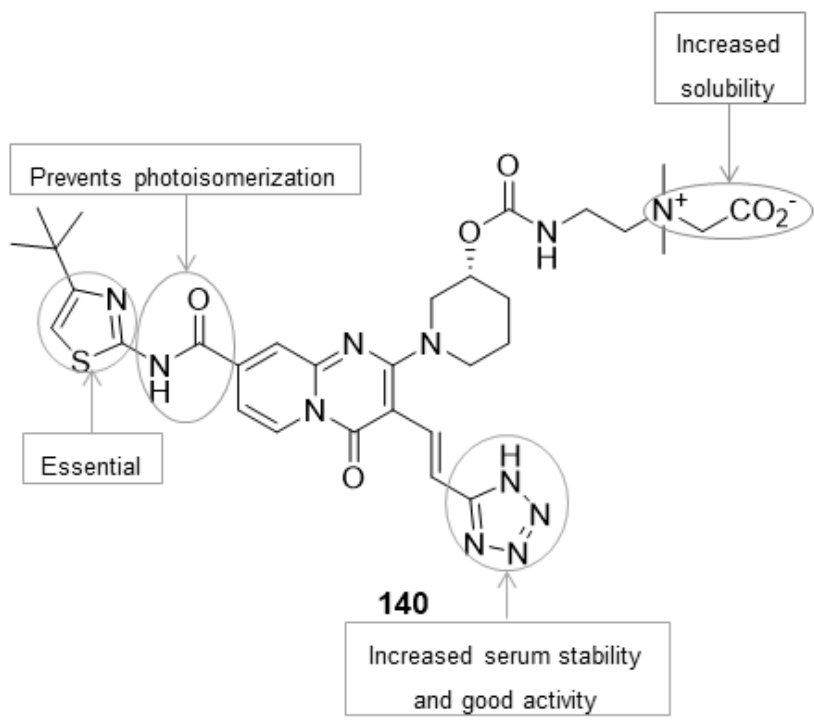

Figure 15 Conclusions from SAR studies for pyridopyrimidine and analogues.

3D Crystal structures of $\mathbf{1 4 0}$ bound to the target, either AcrB or MexB, show that the tert-butyl thiazolyl aminocarboxyl pyridopyrimidine moiety of this compound binds tightly to a narrow depression, designated by hydrophobic trap, close to the deep substrate binding pocket. Additionally, the tetrazole ring and the piperidine acetoamino ethylene ammonioacetate moiety interact with ionic and/or hydrophilic residues in the substrate translocation channel. The piperidine acetoamino ethylene ammonio-acetate moiety also covers part of the minocycline and doxorubicin binding site $[23,28$, $151]$.
Quinoline derivatives with EPI activity include a multiplicity of derivatives, including pyrrido-, alkoxy-, thioalkoxy-, alkylamino- and chloro- derivatives. These compounds were able to restore an intracellular concentration of antibiotic drugs expelled by efflux pumps, as well as to induce the increase in antibiotic susceptibility, acting as competitive inhibitors of the antibiotic flux. In $E$. aerogenes and $K$. pneumoniae, quinolines were more effective than $\mathrm{PA} \beta \mathrm{N}$ (124) in inhibiting the activity of the AcrB pump, potentiating the activity of fluoroquinolones, tetracycline, and chloramphenicol [11, 24, 26]. Among the different classes of substituted quinolines, it was proven that the best side chain was piperidinoethyl, which potentiated efficiently the restoration of drug susceptibility in alkoxy- and thioalkoxyquinolines. The heteroatom connecting the side chain is also crucial for activity, being the most potent the amino substituent, then the thioalkyl-, followed by the oxoderivative. The position of the branched substituted groups is also detrimental for the EPI activity [24]. Branched side chains containing piperidinoethyl and morpholinopropyl groups were associated with the most interesting results, being compound 141, containing a piperidinoethyl side chain, the most active when in combination with chloramphenicol [159]. It has been hypothesized that the pump inhibition is greatly influenced by the branched side chain, and takes place either in the inner-membrane transporter or at the junction between the inner pump and the outer channel [159].

Given these promising results, further research in this kind of compounds was performed. Alkoxyquinolines were synthesized and tested against a strain of $E$. aerogenes overexpressing the AcrAB efflux pump. Compound 142 induced an increase in the susceptibility of chloramphenicol, tetracycline, and fluoroquinolone. This could happen due to interference during active pumping out of the antibiotic [160].

Chloroquinolines also demonstrated efficacy as EPIs, modulating chloramphenicol activity, decreasing its MIC 8fold for resistant strains. Synthesis of analogues led to the conclusion that amino derivatives are more potent than thiol derivatives, pointing out the importance of the side chain for the efflux inhibition. Protonation of the nitrogen could also play an important role at environmental $\mathrm{pH}$, stimulating recognition and binding of the drug to the sites located within the pump's cavity. The heteroatom is also crucial for the activity, being nitrogen the most efficient. Compound 143 is the chloroquinoline with the best results found for the inhibition of the main efflux pump of E. aerogenes [161]. Alkylaminoquinazoline derivatives lacking a nitro group were also evaluated for their ability to decrease chloramphenicol and quinolone efflux in $E$. aerogenes, through the inhibition of AcrAB efflux pump. The results obtained suggest that this family of compounds recognizes the same pump site responsible for the transport of chloramphenicol and quinolones, competing with them. Structurally, it has been shown that a morpholine group with a propyl chain makes a more active alkylaminoquinazoline. 
The most active compound of this family is compound $\mathbf{1 4 4}$ [162].

Benzothiazoles 2-substituted were first tested for the potentiation of ciprofloxacin in AdeABC overexpressing $A$. baumanii. These compounds did not show antibacterial activity when tested alone in bacteria. However, when combined with ciprofloxacin, there was an observation of a reversal in antibacterial susceptibility. The synthesis of several derivatives of this family led to pharmacophore studies, regarding the features of the most promising compounds. Using computational methods, it was revealed that, in order for the compounds to be biologically active, the following features should be present: the nitrogen atom in the thiazole ring and the carbonyl oxygen in the amide function substituted on the second position of the benzothiazole ring must have an hydrogen bonding acceptor property; the benzene ring in the fused ring system, the phenyl group attached to the second position of the benzothiazole ring and the phenyl ring in the 2phenylacetamide and/or 3-phenylpropionamide moiety are essential, since they provide a hydrophobic aromatic property. Of the synthesized compounds, the ones that gathered these features and, therefore, presented the most promising activity, were compounds 145, 146, and 147 [163]. These compounds were also tested for their activity against AcrAB-TolC, in an overexpressing strain of E. coli, reducing the MIC of ciprofloxacin 10-fold, in the case of 146 and 147, and 8-fold, for 145 [164]. Unlike AdeABC, AcrAB-TolC's crystal structure is elucidated, making docking studies possible. This predicted that all three compounds act as AcrB substrates, binding to the distal pocket site in the AcrB porter domain by blocking or inhibiting the ciprofloxacin binding site, generating steric hindrance and thus impeding the binding of the antibiotic. Furthermore, 146 and 147 showed stronger binding interactions than ciprofloxacin [164].

Indole derivatives have been useful in inhibiting protein targets of virus, tumors and bacteria. Since small molecules with conjugated aromatic rings, in which indoles fit, present high potential as EPIs, efforts were placed in synthesizing derivatives that presented this kind of activity. The TolC 3D structure has already been disclosed, making possible the structure-based design of inhibitors. Indoles 148 and 149 were proven efficient inhibitors of TolC in $E$. coli, potentiating the effects of chloramphenicol, tetracycline, erythromycin, and ciprofloxacin, with decreased MIC values of 2-fold for tetracycline and erythromycin and 8-fold for the other two [165].

\subsubsection{Existing Drugs}

Other drugs used for treatments other than antibacterial have demonstrated EPI activity. Tetracycline resistance in $P$. aeruginosa was reduced 8-fold when the phenothiazine fluphenazine, an antipsychotic, was present. Chlorpromazine (150) also showed EPI-like activity for the BpeAB-OprB system in Burkholderia pseudomallei and a reduction of the expression of $a c r B$ in $E$. coli and $S$. enterica samovar
Typhimurium, suggesting an inhibition of AcrB production $[11,27,128]$.

Selective serotonin-reuptake inhibitors have been studied for their ability to act as EPIs for RND efflux pumps in E. coli. It was found that sertraline (151) was capable of potentiating the activity of fluoroquinolones. However, its activity is limited, since it is possible that this compound also acts as an inductor of efflux pumps. Studies have shown that this compound could accumulate in tissues or cells, and therefore, sertraline's (151) concentration may be higher than that measured in plasma. This means that a standard dose of this drug could enhance antibiotic efficacy. It has not yet been studied if a sertraline (151) metabolite could be administered at a dose higher than sertraline. However, it was hypothesized that it could have a better EPI activity, while causing less undesired effects on the central nervous system [166].

Through the observation of previously reported EPIs, Piddock et al. (2010) suggested that simple heterocyclic nitrogen-containing compounds could also act as EPIs and synergize with ciprofloxacin in strains of $S$. enterica serovar Typhimurium that overexpressed the AcrAB-TolC efflux pump [167]. It has been shown that compounds containing a phenylethylamine moiety, as $\mathrm{PA} \beta \mathrm{N}$ (126) does, such as epinephrine, norepinephrine, and cathinone, are able to potentiate the activity of ciprofloxacin. Of the compounds tested, trimethoprim (152) and epinephrine (153), showed the best results for the tested strains, which included, apart from $S$. enterica serovar Typhimurium, $P$. aeruginosa, E. coli, and $K$. pneumoniae. These compounds showed synergy for the strains where AcrAB-TolC was active, but showed no activity when the pump was inactive. Thus, it can be hypothesized that these compounds either inhibit the expression of the genes encoding AcrAB-TolC, or interact directly with the pump [167]. Both compounds possess structural features similar to previously described EPIs, such as PAßN (124), L-Orn-L-hPhe-3-NHQ (125), and NMP (136), such as an aromatic ring linked to a basic nitrogen, in the case of epinephrine (153), or a pyrimidine ring, for trimethoprim (152). Trimethoprim (152) is already used in combination with another antibiotic, sulfamethoxazole, and combination with a quinolone was hypothesized to be a viable choice. On the other hand, a combination of epinephrine (153) with an antibiotic does not seem a viable option, because of the likelihood of the occurrence of sympathomimetic effects [167].

The antimalarial artesunate (154) has also shown EPI activity, enhancing the effects of $\beta$-lactam antibiotics, such as penicillin $\mathrm{G}$, oxacillin, and ampicillin, and novobiocin against $E$. coli. The mechanism through which 154 expresses its activity is by the inhibition of the expression of the AcrAB-TolC system, not exhibiting antibiotic activity by itself [168].

\section{SMR}

The SMR family comprises the smallest drug efflux proteins known, possessing only four transmembrane segments in its 
composition, and are exclusive to bacteria. They are involved in the efflux of lipophilic compounds, such as quaternary ammonium salts and a variety of antibiotics. These pumps also use an electrochemical proton gradient in order to exert its efflux activity [7, 32, 38, 169].

One example of a SMR-type pump is the EmrE transporter (Figure 16, PDB 3B61), present in E. coli. In the absence of the ligand, the asymmetric unit of EmrE contains eight EmrE monomers, with four transmembrane helices composing each of them. If a ligand is present, it can function as an inverted homodimer [7, 170].

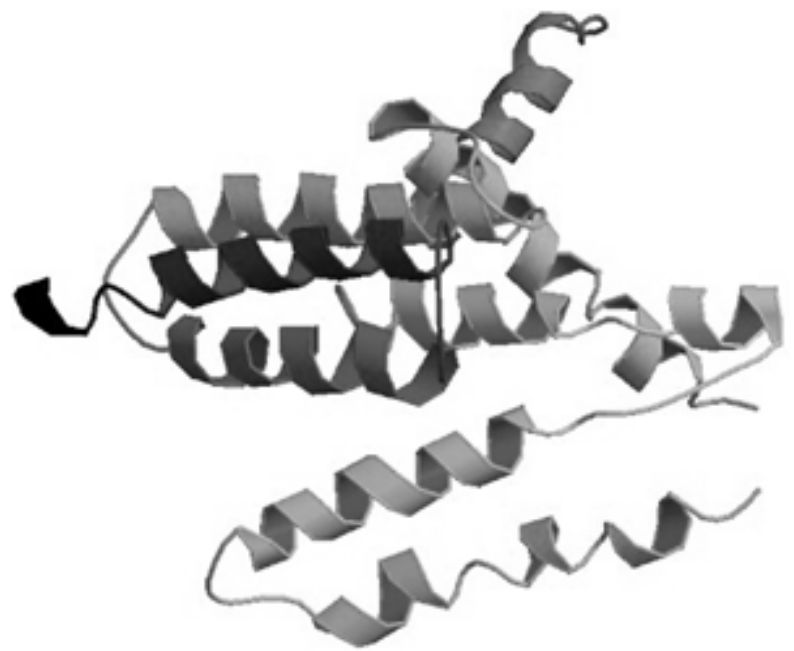

Figure 16 Crystal structure of EmrE (PDB 3B61) of E. coli.

There have not been many studies concerning inhibitors of this family of efflux pumps, and the few that have been performed were only in silico. Figure 17 shows the structures of the SMR inhibitors that will be further discussed.<smiles>O=c1c(O)c(-c2ccc(O)c(O)c2)oc2cc(O)cc(O)c12</smiles>

155 with two pumps belonging to the SMR family, the Mmr, in Mycobacterium smegmatis, and the EmrE pumps, in E. coli, were carried out. In Mmr, the hydroxyl groups present in the 3' and 4' positions of the B ring of quercetin were predicted to interact with Glu-14 in the helix 1, and the keto group of the hydroxychromen ring of the ligand interacts with the Trp-48 of the helix 1. Additionally, hydrophobic interactions were shown by the residues Phe-44, Tyr-40 and Trp-48, and $\pi-\pi$ interactions were found with Phe-44. Furthermore, 155 was also predicted of interacting with dimeric forms of the protein [171]. In the case of EmrE, the interaction between the 3' and 4' hydroxyls with Glu-14 can also be observed, as can the hydroxyl in the C-7 position with Tyr-60. Hydrophobic interactions are present, with the residues Ile11, Ser-64, Gly-67 and Leu-70, and $\pi-\pi$ interactions exist with the residues Tyr-60 and Trp-63. Structural studies suggest that EmrE is mostly in the dimer state, which also interacts with quercetin (155) [171].

\subsubsection{Synthetic Compounds}

Cation ligands were subjected to docking studies with the Mmr protein of the SMR family in Mycobacterium tuberculosis and the EmrE dimer from $E$. coli. The rationale behind this study is the fact that SMR can extrude cations. This study led to the identification of new virtual leads, amongst which is compound 156. ADME studies prove that all the molecules present in this study obey the Lipinski rule of five and the Jorgenson rule of three, which shows that the compounds possess good drug-like properties [172].

\section{MATE}

MATE transporters are responsible for the efflux of cationic,

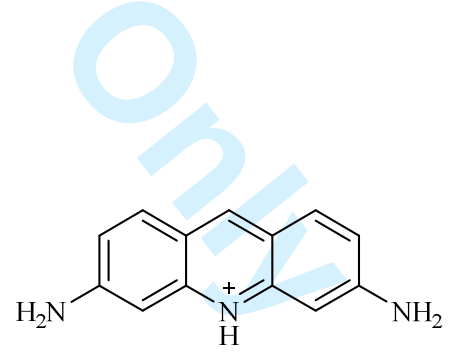

156

Figure 17 Structures of SMR inhibitors 155 and 156.

\subsection{SMR inhibitors}

\subsubsection{Natural Products}

Quercetin (155) is a flavonol, present in many plants. This compound is very well tolerated in humans, as are its glycoconjugates, and presents a multiplicity of activities, such as antioxidant, anti-inflammatory and antimycobacterial. Only docking studies of this compound lipophilic substances, providing bacteria and cancer cells with MDR, using $\mathrm{Na}^{+}$or $\mathrm{H}^{+}$influx. They have 12 transmembrane helices, constituted by 400 to 700 amino acids. The mechanism of efflux is thought to happen by a rocker-switch mechanism. Members of the MATE family include the MepA (Figure 18, PDB 1TZP) transporter, in $S$. aureus, even though seventeen MATE proteins have been identified in eleven species, capable of extruding antibiotics. Ethidium bromide, tetraphenylphosphonium, acriflavine, 
berberine, and norfloxacin have been reported as MATE substrates [38, 173-181].

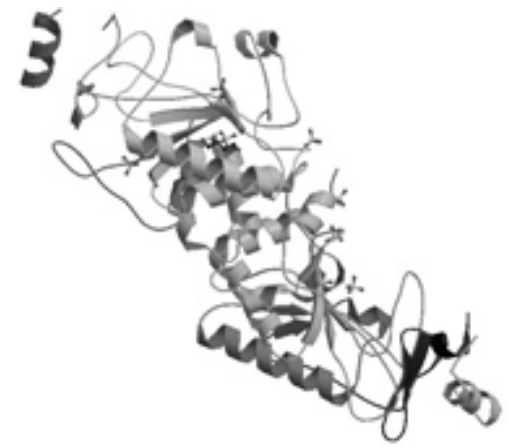

Figure 18 Crystal structure of MepA (PDB 1TZP) of E. coli in its inactive form.

In Figure 19 are compiled the structures of the compounds that have so far proven their usefulness as MATE inhibitors.

\subsection{MATE inhibitors}

\subsubsection{Natural Products}

A study performed by Kesherwani et al. (2017) accomplished the docking of phytochemicals with the NorM transporter, belonging to the MATE family [182]. After a screening of a library of natural products, three hits were identified: derhamnosyl suspenaside (157), prunin 7' $-O$ gallate (158), and quercetin diglucoside (159). These were the best compounds, as their results in binding free energy calculation were the most favorable. When binding free energy decomposition and hydrogen and hydrophobic statistics were taken into account, $\mathbf{1 5 8}$ was elected the best hit compound. Furthermore, this compound was stable during the simulation. Overall, it has been suggested that the compounds which interact towards the cation binding residue in the central cavity of NorM will have better results, and that good inhibitors should have interaction with a few key residues, Gln-34, Val-35, Gly-38, Phe-63, Tyr-67, Ile68, Glu-261, Tyr-294, and Asp-377, which will lead to<smiles>O=C(/C=C/c1ccc(O)c(O)c1)O[C@@H]1[C@@H](O)[C@H](O)[C@H](OC[C@H](O)c2ccc(O)c(O)c2)O[C@@H]1CO</smiles>

157<smiles>O=c1c(O[C@@H]2O[C@H](CO)[C@@H](O)[C@H](O)[C@H]2O[C@@H]2O[C@H](O)[C@@H](O)[C@H](O)[C@H]2O)c(-c2ccc(O)c(O)c2)oc2cc(O)cc(O)c12</smiles>

159<smiles>O=C(OC[C@H]1O[C@@H](Oc2cc(O)c3c(c2)O[C@@H](c2ccc(O)cc2)CC3=O)[C@H](O)[C@@H](O)[C@@H]1O)c1cc(O)c(O)c(O)c1</smiles>

158

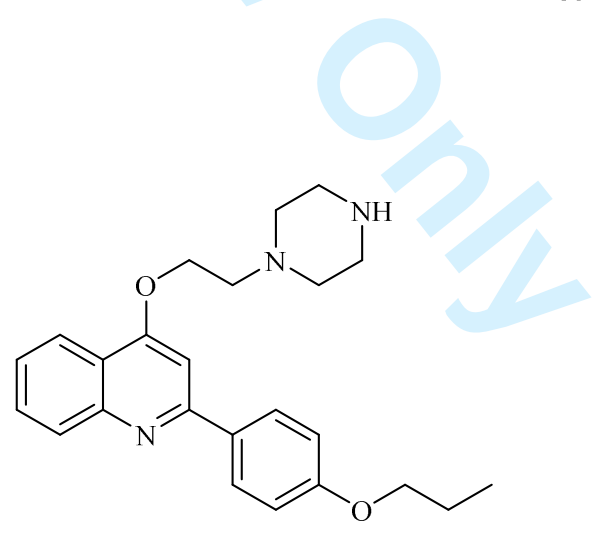

160<smiles>Clc1ccc(Br)cc1C=C[C@H]1[CH]CNCC1</smiles>

162

Figure 19 Structures of MATE inhibitors $157-162$.<smiles>ClC1=C(OC[C@H]2[CH]CCNC2)C=C(Br)CC1</smiles>

161 
maximum stability and affinity with the NorM transporter [182].

\subsubsection{Synthetic Compounds}

These compounds have been previously described as MFS inhibitors, namely of the NorA pump. However, similar activity has been described for the MepA pump, of the MATE family. Compound $\mathbf{8 0}$ (Figure 5), incorporating a bisaryl urea moiety at the 7-position, was able to successfully inhibit approximately $84 \%$ of ethidium bromide efflux in a MepA overexpressing strain of S.aureus [104]. Phenylquinoline derivatives also proved efficient against the same strain. Differently from what was observed with NorA, the compounds that inhibited MepA most efficiently were 86 (Figure 5) and 160 (Figure 19), which were able to reduce ethidium bromide's MIC by 16-fold at concentrations of 0.78 and $1.56 \mu \mathrm{g} / \mathrm{ml}$, respectively, in the MepA overexpressing strain. Ethidium bromide was chosen, instead of ciprofloxacin, as it is a better MepA substrate and other derivatives were able to inhibit both NorA and MepA efflux pumps, being able to completely restore the activity of ciprofloxacin and ethidium bromide against strains overexpressing such pumps [107].

Paroxetine derivatives (104), effective against the NorA pump, also demonstrated potency in inhibiting the MepA pump in $S$. aureus. Oppositely to NorA, paroxetine derivatives do not need the fluorophenyl ring at position 4 to be MepA EPIs. In fact, 4-unsubstituted analogues proved to be more potent than 4-F-phenyl derivatives in inhibiting the MepA pump, as is the case of compound 161 and 162 [122].

\section{CONCLUSIONS}

Efflux pumps are one of the main causes for antimicrobial resistance. Their inhibition can lead to the restoration of bacteria susceptibility and antibiotic efficacy. Throughout this review, the data presented leads to the conclusion that EPIs are promising compounds and can help reversing the problem of antimicrobial resistance. However, the research into these kind of compounds is still in a very preliminary state, since no EPI is yet under clinical trials.

Even though no general structural features can be pointed out for a molecule to be an EPI, it can be observed that small molecules with high lipophilicity are most likely to fulfill the requirements to inhibit efflux pumps. Hydrophilic compounds can also be transported through porins present in the membrane. Heterocyclic compounds also show potential as EPIs, mainly nitrogen heterocycles, but also fluorine and boron heteroatoms, since the likelihood of bacterial exposure to these atoms is very low. The basis for the MDR pumps inhibition has to do with the penetration rules, and whether or not a compound is capable of penetrating the bacterial membrane. Therefore, a library of compounds that are able to do that would be something that would bring advances to this study field.

One of the main aims of this review is to present SAR studies, so that pharmaceutical chemists can refine molecular structures, leading to the achievement of more potent and specific compounds. The use of synthetic approaches, either to modify molecules obtained from natural sources, or drugs that are already in therapeutic use, or even to develop novel compounds, is vital for these SAR studies. The search of EPIs derived from natural products comes from the fact that plants need to protect themselves from pathogenic microorganisms, and produce metabolites that act as antimicrobial and EPIs, that synergize with each other. However, the referred compounds are not specific for the pump they have been studied, showing a multitarget character: flavonoids, coumarins, cinnamic acids, among others, present a multiplicity of activities already described, suggesting their promiscuity.

The advantage of assays with existing drugs lies in their safety profile and, most times, their clinical use for combination therapy has been characterized. In some cases, the compounds were even investigated as inhibitors of human efflux pumps, for cancer treatment. This can be regarded as an advantage, leading to a new class of drugs with dual human/bacterial EPI, particularly useful in cancer patients with infections, potentiating the effects of both the anticancer and the antibacterial drugs.

The future in studies in bacterial EPIs should lie either in the search for selectivity for the bacterial target, or in the disclosure of the common features of the universal bacterial EPI, that can successfully target all efflux pumps. The SAR studies gathered herein with the 3D structural information available are expected to accelerate the structure-based design of new EPIs, founded on the existing models.

\section{LIST OF ABBREVIATIONS}

3D-QSAR: Three-dimensional quantitative structure activity relationship; ABC: ATP-binding cassette; ADME: Absorption, distribution, metabolism and excretion; ATP: Adenosine triphosphate; BCRP: Breast cancer resistance protein; COX-2: Ciclooxigenase-2; CYP450: Cytochrome P450; EPI: Efflux pump inhibitor; $\mathrm{IC}_{50}$ : Half maximal inhibitory concentration; MATE: Multidrug and toxic compound extrusion; MDR: Multidrug resistance; MIC: Minimum inhibitory concentration; MFS: Major facilitator superfamily; MRP: Multidrug resistance protein; MRSA: Methicillin-resistant Staphylococcus aureus; NDGA: Nordihydroguaretic acid; NMP: 1-(1-Naphthylmethyl)piperazine; P-gp: P-Glycoprotein; PA $\beta \mathrm{N}$ : Phenyl-arginine $\beta$ naphtylamide; PDB: Protein Data Bank; PSSRI: Phenylpiperidine selective serotonin reuptake inhibitors; RND: Resistance-nodulation-division; SAR: Structure activity relationship; SMR: Small multidrug resistance.

\section{ACKNOWLEDGEMENTS}

This work was supported through national funds provided by FCT/MCTES - Foundation for Science and Technology from the Minister of Science, Technology and Higher Education (PIDDAC) and European Regional Development Fund 
(ERDF) through the COMPETE - Programa Operacional Factores de Competitividade (POFC) programme, under the project PTDC/MAR-BIO/4694/2014 (reference POCI-010145-FEDER-016790; Project 3599 - Promover a Produção Científica e Desenvolvimento Tecnológico e a Constituição de Redes Temáticas (3599-PPCDT)) and PTDC/AAGTEC/0739/2014 (POCI-01-0145-FEDER-016793, Projeto 9471) in the framework of the programme PT2020 and by the project INNOVMAR - Innovation and Sustainability in the Management and Exploitation of Marine Resources (reference NORTE-01-0145-FEDER-000035, within Research Line NOVELMAR), supported by North Portugal Regional Operational Programme (NORTE 2020), under the PORTUGAL 2020 Partnership Agreement, through the ERDF and was partially supported by the Strategic Funding UID/Multi/04423/2013 through national funds provided by FCT, and ERDF, in the framework of the programme PT2020.

\section{REFERENCES}

[1] Davies, J.; Davies, D., Origins and Evolution of Antibiotic Resistance. Microbiol Mol Biol Rev 2010, 74 (3), 417-433.

[2] Alanis, A. J., Resistance to antibiotics: are we in the postantibiotic era? Arch Med Res 2005, 36 (6), 697-705.

[3] Ventola, C. L., The Antibiotic Resistance Crisis: Part 1: Causes and Threats. P T 2015, 40 (4), 277-283.

[4] Levy, S. B.; Marshall, B., Antibacterial resistance worldwide: causes, challenges and responses. Nat Med 2004, 10 (12 Suppl), S122-9.

[5] Munita, J. M.; Arias, C. A., Mechanisms of Antibiotic Resistance. Microbiol Spect 2016, 4 (2), 10.1128/microbiolspec.VMBF-0016-2015.

[6] Chattopadhyay, M. K.; Jagannadham, M. V., Vesiclesmediated resistance to antibiotics in bacteria. FrontMicrobiol 2015, 6, 758.

[7] Sun, J.; Deng, Z.; Yan, A., Bacterial multidrug efflux pumps: mechanisms, physiology and pharmacological exploitations. Biochem Biophys Res Commun 2014, 453 (2), 254-67.

[8] Blanco, P.; Hernando-Amado, S.; Reales-Calderon, J. A.; Corona, F.; Lira, F.; Alcalde-Rico, M.; Bernardini, A.; Sanchez, M. B.; Martinez, J. L., Bacterial Multidrug Efflux Pumps: Much More Than Antibiotic Resistance Determinants. Microorganisms 2016, 4 (1).

[9] Fernandez, L.; Hancock, R. E., Adaptive and mutational resistance: role of porins and efflux pumps in drug resistance. Clin Microbiol Rev 2012, 25 (4), 661-81.

[10] Webber, M. A.; Piddock, L. J. V., The importance of efflux pumps in bacterial antibiotic resistance. J Antimicrob Chemother 2003, 51 (1), 9-11.

[11] Tegos, G. P.; Haynes, M.; Strouse, J. J.; Khan, M. M.; Bologa, C. G.; Oprea, T. I.; Sklar, L. A., Microbial efflux pump inhibition: tactics and strategies. Curr Pharm Des 2011, 17 (13), 1291-302.

[12] Van Bambeke, F.; Pages, J. M.; Lee, V. J., Inhibitors of bacterial efflux pumps as adjuvants in antibiotic treatments and diagnostic tools for detection of resistance by efflux. Recent Pat Antiinfect Drug Discov 2006, 1 (2), 157-75.

[13] Marquez, B., Bacterial efflux systems and efflux pumps inhibitors. Biochimie 2005, 87 (12), 1137-47.

[14] Kumar, R.; Patial, S., A Review on Efflux Pump Inhibitors of Gram-Positive and Gram-Negative Bacteria from Plant Sources. Int J Curr Microbiol App Sci 2016, 5 (6), 834-855.

[15] Rana, T.; Singh, S.; Kaur, N.; Pathania, K.; Farooq, U., A Review on Efflux Pump Inhibitors of Medically Important Bacteria from Plant Sources. Int J Pharm Sci Rev Res 2014, 26 (2), 101-111.

[16] Prasch, S.; Bucar, F., Plant derived inhibitors of bacterial efflux pumps: an update. Phytochem Rev 2015, 14 (6), 961-974.

[17] Zhang, L.; Ma, S., Efflux pump inhibitors: a strategy to combat P-glycoprotein and the NorA multidrug resistance pump. Chem Med Chem 2010, 5 (6), 811-22.

[18] Schindler, B. D.; Jacinto, P.; Kaatz, G. W., Inhibition of drug efflux pumps in Staphylococcus aureus: current status of potentiating existing antibiotics. Future Microbiol 2013, 8 (4), 491-507.

[19] Kumar, S.; Mukherjee, M. M.; Varela, M. F., Modulation of Bacterial Multidrug Resistance Efflux Pumps of the Major Facilitator Superfamily. Int $J$ Bacteriol 2013, 2013.

[20] Handzlik, J.; Matys, A.; Kiec-Kononowicz, K., Recent Advances in Multi-Drug Resistance (MDR) Efflux Pump Inhibitors of Gram-Positive Bacteria S. aureus. Antibiotics (Basel) 2013, 2 (1), 28-45.

[21] Stavri, M.; Piddock, L. J. V.; Gibbons, S., Bacterial efflux pump inhibitors from natural sources. J Antimicrob Chemother 2007, 59 (6), 1247-1260.

[22] Gibbons, S., Plants as a Source of Bacterial Resistance Modulators and Anti-Infective Agents. Phytochem Rev 2005, 4 (1), 63-78.

[23] Ruggerone, P.; Murakami, S.; Pos, K. M.; Vargiu, A. V., RND efflux pumps: structural information translated into function and inhibition mechanisms. Curr Top Med Chem 2013, 13 (24), 3079-100.

[24] Pagès, J.-M.; Masi, M.; Barbe, J., Inhibitors of efflux pumps in Gram-negative bacteria. Trends Mol Med 2005, 11 (8), 382-389.

[25] Opperman, T.; Nguyen, S., Recent advances toward a molecular mechanism of efflux pump inhibition. Front Microbiol 2015, 6 (421).

[26] Martins, M.; Dastidar, S. G.; Fanning, S.; Kristiansen, J. E.; Molnar, J.; Pages, J. M.; Schelz, Z.; Spengler, G.; Viveiros, M.; Amaral, L., Potential role of non-antibiotics (helper compounds) in the treatment of multidrug-resistant Gram-negative infections: mechanisms for their direct and indirect activities. Int J Antimicrob Agents 2008, 31 (3), 198208.

[27] Blair, J. M. A.; Piddock, L. J. V., Structure, function and inhibition of RND efflux pumps in Gram-negative bacteria: an update. Curr Opin Microbiol 2009, 12 (5), 512519. 
[28] Aron, Z.; Opperman, T. J., Optimization of a novel series of pyranopyridine RND efflux pump inhibitors. Curr Opin Microbiol 2016, 33, 1-6.

[29] Venter, H.; Mowla, R.; Ohene-Agyei, T.; Ma, S., RNDtype drug efflux pumps from Gram-negative bacteria: molecular mechanism and inhibition. Front Microbiol 2015, 6, 377.

[30] Song, L.; Wu, X., Development of efflux pump inhibitors in antituberculosis therapy. Int $J$ Antimicrob Agents 2016, 47 (6), 421-9.

[31] Pule, C. M.; Sampson, S. L.; Warren, R. M.; Black, P. A.; van Helden, P. D.; Victor, T. C.; Louw, G. E., Efflux pump inhibitors: targeting mycobacterial efflux systems to enhance TB therapy. J Antimicrob Chemother 2016, 71 (1), 17-26.

[32] Zechini, B.; Versace, I., Inhibitors of multidrug resistant efflux systems in bacteria. Recent Pat Antiinfect Drug Discov 2009, 4 (1), 37-50.

[33] El-Awady, R.; Saleh, E.; Hashim, A.; Soliman, N.; Dallah, A.; Elrasheed, A.; Elakraa, G., The Role of Eukaryotic and Prokaryotic ABC Transporter Family in Failure of Chemotherapy. Front Pharmacol 2016, 7, 535.

[34] Silva, R.; Vilas-Boas, V.; Carmo, H.; Dinis-Oliveira, R. J.; Carvalho, F.; de Lourdes Bastos, M.; Remião, F., Modulation of P-glycoprotein efflux pump: induction and activation as a therapeutic strategy. Pharmacol Ther 2015, 149, 1-123.

[35] Palmeira, A.; Rodrigues, F.; Sousa, E.; Pinto, M.; Vasconcelos, M. H.; Fernandes, M. X., New uses for old drugs: pharmacophore-based screening for the discovery of P-glycoprotein inhibitors. Chem Biol Drug Des 2011, 78 (1), 57-72.

[36] Lopes-Rodrigues, V.; Sousa, E.; Vasconcelos, M. H., Curcumin as a Modulator of P-Glycoprotein in Cancer: Challenges and Perspectives. Pharmaceuticals (Basel) 2016, 9 (4).

[37] Lopes-Rodrigues, V.; Seca, H.; Sousa, D.; Sousa, E.; Lima, R. T.; Vasconcelos, M. H., The network of Pglycoprotein and microRNAs interactions. Int $J$ Cancer 2014, 135 (2), 253-63.

[38] Jang, S., Multidrug efflux pumps in Staphylococcus aureus and their clinical implications. $J$ Microbiol 2016, 54 (1), 1-8.

[39] Locher, K. P., Mechanistic diversity in ATP-binding cassette (ABC) transporters. Nat Struct Mol Biol 2016, 23 (6), 487-93.

[40] Wilkens, S., Structure and mechanism of ABC transporters. F1000Prime Rep 2015, 7, 14.

[41] Taylor, A. B.; Benglis, J. D. M.; Dhandayuthapani, S.; Hart, P. J., Structure of Mycobacterium tuberculosis Methionine Sulfoxide Reductase A in Complex with ProteinBound Methionine. J Bacteriol 2003, 185 (14), 4119-4126.

[42] Oluwatuyi, M.; Kaatz, G. W.; Gibbons, S., Antibacterial and resistance modifying activity of Rosmarinus officinalis. Phytochemistry 2004, 65 (24), 3249-54.

[43] Hussein, A. A.; Rodriguez, B., Isopimarane diterpenoids from Lycopus europaeus. J Nat Prod 2000, 63 (3), 419-21.
[44] Gibbons, S.; Oluwatuyi, M.; Veitch, N. C.; Gray, A. I., Bacterial resistance modifying agents from Lycopus europaeus. Phytochemistry 2003, 62 (1), 83-7.

[45] Upadhyay, H. C.; Dwivedi, G. R.; Roy, S.; Sharma, A.; Darokar, M. P.; Srivastava, S. K., Phytol derivatives as drug resistance reversal agents. Chem Med Chem 2014, 9 (8), 1860-8.

[46] Dwivedi, G. R.; Maurya, A.; Yadav, D. K.; Khan, F.; Darokar, M. P.; Srivastava, S. K., Drug Resistance Reversal Potential of Ursolic Acid Derivatives against Nalidixic Acidand Multidrug-resistant Escherichia coli. Chem Biol Drug Des 2015, 86 (3), 272-83.

[47] Wang, S. Y.; Sun, Z. L.; Liu, T.; Gibbons, S.; Zhang, W. J.; Qing, M., Flavonoids from Sophora moorcroftiana and their synergistic antibacterial effects on MRSA. Phytother Res 2014, 28 (7), 1071-6.

[48] Chan, B. C.; Ip, M.; Gong, H.; Lui, S. L.; See, R. H.; Jolivalt, C.; Fung, K. P.; Leung, P. C.; Reiner, N. E.; Lau, C. B., Synergistic effects of diosmetin with erythromycin against ABC transporter over-expressed methicillin-resistant Staphylococcus aureus (MRSA) RN4220/pUL5054 and inhibition of MRSA pyruvate kinase. Phytomedicine 2013, 20 (7), 611-4.

[49] Dwivedi, G. R.; Upadhyay, H. C.; Yadav, D. K.; Singh, V.; Srivastava, S. K.; Khan, F.; Darmwal, N. S.; Darokar, M. P., 4-Hydroxy- $\alpha$-tetralone and its derivative as drug resistance reversal agents in multi drug resistant Escherichia coli. Chem Biol Drug Des 2014, 83 (4), 482-92.

[50] Chan, B. C.; Han, X. Q.; Lui, S. L.; Wong, C. W.; Wang, T. B.; Cheung, D. W.; Cheng, S. W.; Ip, M.; Han, S. Q.; Yang, X. S.; Jolivalt, C.; Lau, C. B.; Leung, P. C.; Fung, K. P., Combating against methicillin-resistant Staphylococcus aureus - two fatty acids from Purslane (Portulaca oleracea L.) exhibit synergistic effects with erythromycin. J Pharm Pharmacol 2015, 67 (1), 107-16.

[51] Maurya, A.; Dwivedi, G. R.; Darokar, M. P.; Srivastava, S. K., Antibacterial and synergy of clavine alkaloid lysergol and its derivatives against nalidixic acid-resistant Escherichia coli. Chem Biol Drug Des 2013, 81 (4), 484-90. [52] Gibbons, S.; Oluwatuyi, M.; Kaatz, G. W., A novel inhibitor of multidrug efflux pumps in Staphylococcus aureus. J Antimicrob Chemother 2003, 51 (1), 13-7.

[53] Pezza, R. J.; Villarreal, M. A.; Montich, G. G.; Argaraña, C. E., Vanadate inhibits the ATPase activity and DNA binding capability of bacterial MutS. A structural model for the vanadate-MutS interaction at the Walker A motif. Nucleic Acids Res 2002, 30 (21), 4700-4708.

[54] Yan, N., Structural advances for the major facilitator superfamily (MFS) transporters. Trends in Biochem Sci 2013, 38 (3), 151-159.

[55] Yin, Y.; He, X.; Szewczyk, P.; Nguyen, T.; Chang, G., Structure of the Multidrug Transporter EmrD from Escherichia coli. Science (New York, N.Y.) 2006, 312 (5774), 741-744.

[56] Yan, N., Structural Biology of the Major Facilitator Superfamily Transporters. Annu Rev Biophys 2015, 44, 257 83. 
[57] Tamura, N.; Konishi, S.; Yamaguchi, A., Mechanisms of drug/H+ antiport: complete cysteine-scanning mutagenesis and the protein engineering approach. Curr Opin Chem Biol 2003, 7 (5), 570-579.

[58] Coelho, M. L.; Ferreira, J. H.; de Siqueira Junior, J. P.; Kaatz, G. W.; Barreto, H. M.; de Carvalho Melo Cavalcante, A. A., Inhibition of the NorA multi-drug transporter by oxygenated monoterpenes. Microb Pathog 2016, 99, 173177.

[59] Thota, N.; Koul, S.; Reddy, M. V.; Sangwan, P. L.; Khan, I. A.; Kumar, A.; Raja, A. F.; Andotra, S. S.; Qazi, G. N., Citral derived amides as potent bacterial NorA efflux pump inhibitors. Bioorg Med Chem 2008, 16 (13), 6535-43. [60] Smith, E. C.; Williamson, E. M.; Wareham, N.; Kaatz, G. W.; Gibbons, S., Antibacterials and modulators of bacterial resistance from the immature cones of Chamaecyparis lawsoniana. Phytochemistry 2007, 68 (2), 210-7.

[61] Smith, E. C.; Kaatz, G. W.; Seo, S. M.; Wareham, N.; Williamson, E. M.; Gibbons, S., The phenolic diterpene totarol inhibits multidrug efflux pump activity in Staphylococcus aureus. Antimicrob Agents Chemother 2007, 51 (12), 4480-3.

[62] Zhang, J.; Sun, Y.; Wang, Y.; Lu, M.; He, J.; Liu, J.; Chen, Q.; Zhang, X.; Zhou, F.; Wang, G.; Sun, X., Nonantibiotic agent ginsenoside $20(S)-\mathrm{Rh} 2$ enhanced the antibacterial effects of ciprofloxacin in vitro and in vivo as a potential NorA inhibitor. Eur J Pharmacol 2014, 740, 277 84.

[63] Zhou, S.; Schuetz, J. D.; Bunting, K. D.; Colapietro, A. M.; Sampath, J.; Morris, J. J.; Lagutina, I.; Grosveld, G. C.; Osawa, M.; Nakauchi, H.; Sorrentino, B. P., The ABC transporter Bcrp1/ABCG2 is expressed in a wide variety of stem cells and is a molecular determinant of the sidepopulation phenotype. Nat Med 2001, 7 (9), 1028-34.

[64] Doyle, L.; Ross, D. D., Multidrug resistance mediated by the breast cancer resistance protein BCRP (ABCG2). Oncogene 2003, 22 (47), 7340-58.

[65] Ahmed, M.; Borsch, C. M.; Neyfakh, A. A.; Schuldiner, S., Mutants of the Bacillus subtilis multidrug transporter Bmr with altered sensitivity to the antihypertensive alkaloid reserpine. J Biol Chem 1993, 268 (15), 11086-9.

[66] Schmitz, F. J.; Fluit, A. C.; Luckefahr, M.; Engler, B.; Hofmann, B.; Verhoef, J.; Heinz, H. P.; Hadding, U.; Jones, M. E., The effect of reserpine, an inhibitor of multidrug efflux pumps, on the in vitro activities of ciprofloxacin, sparfloxacin and moxifloxacin against clinical isolates of Staphylococcus aureus. J Antimicrob Chemother 1998, 42 (6), 807-10.

[67] Markham, P. N.; Westhaus, E.; Klyachko, K.; Johnson, M. E.; Neyfakh, A. A., Multiple Novel Inhibitors of the NorA Multidrug Transporter of Staphylococcus aureus. Antimicrob Agents Chemother 1999, 43 (10), 2404-8.

[68] Kumar, A.; Khan, I. A.; Koul, S.; Koul, J. L.; Taneja, S. C.; Ali, I.; Ali, F.; Sharma, S.; Mirza, Z. M.; Kumar, M.; Sangwan, P. L.; Gupta, P.; Thota, N.; Qazi, G. N., Novel structural analogues of piperine as inhibitors of the NorA efflux pump of Staphylococcus aureus. J Antimicrob Chemother 2008, 61 (6), 1270-6.

[69] Sangwan, P. L.; Koul, J. L.; Koul, S.; Reddy, M. V.; Thota, N.; Khan, I. A.; Kumar, A.; Kalia, N. P.; Qazi, G. N., Piperine analogs as potent Staphylococcus aureus NorA efflux pump inhibitors. Bioorg Med Chem 2008, 16 (22), 9847-57.

[70] Nargotra, A.; Sharma, S.; Koul, J. L.; Sangwan, P. L.; Khan, I. A.; Kumar, A.; Taneja, S. C.; Koul, S., Quantitative structure activity relationship (QSAR) of piperine analogs for bacterial NorA efflux pump inhibitors. Eur J Med Chem 2009, 44 (10), 4128-35.

[71] Stermitz, F. R.; Lorenz, P.; Tawara, J. N.; Zenewicz, L. A.; Lewis, K., Synergy in a medicinal plant: antimicrobial action of berberine potentiated by 5'-methoxyhydnocarpin, a multidrug pump inhibitor. Proc Natl Acad Sci U S A 2000, 97 (4), 1433-7.

[72] Guz, N. R.; Stermitz, F. R.; Johnson, J. B.; Beeson, T. D.; Willen, S.; Hsiang, J.; Lewis, K., Flavonolignan and flavone inhibitors of a Staphylococcus aureus multidrug resistance pump: structure-activity relationships. $\mathrm{J} \mathrm{Med}$ Chem 2001, 44 (2), 261-8.

[73] Bame, J. R.; Graf, T. N.; Junio, H. A.; Bussey, R. O., 3rd; Jarmusch, S. A.; El-Elimat, T.; Falkinham, J. O., 3rd; Oberlies, N. H.; Cech, R. A.; Cech, N. B., Sarothrin from Alkanna orientalis is an antimicrobial agent and efflux pump inhibitor. Planta Med 2013, 79 (5), 327-9.

[74] Morel, C.; Stermitz, F. R.; Tegos, G.; Lewis, K., Isoflavones as potentiators of antibacterial activity. $J$ Agric Food Chem 2003, 51 (19), 5677-9.

[75] Chan, B. C.; Ip, M.; Lau, C. B.; Lui, S. L.; Jolivalt, C.; Ganem-Elbaz, C.; Litaudon, M.; Reiner, N. E.; Gong, H.; See, R. H.; Fung, K. P.; Leung, P. C., Synergistic effects of baicalein with ciprofloxacin against NorA over-expressed methicillin-resistant Staphylococcus aureus (MRSA) and inhibition of MRSA pyruvate kinase. $J$ Ethnopharmacol 2011, 137 (1), 767-73.

[76] Maia, G. L.; Falcao-Silva Vdos, S.; Aquino, P. G.; de Araujo-Junior, J. X.; Tavares, J. F.; da Silva, M. S.; Rodrigues, L. C.; de Siqueira-Junior, J. P.; Barbosa-Filho, J. M., Flavonoids from Praxelis clematidea R.M. King and Robinson modulate bacterial drug resistance. Molecules 2011, 16 (6), 4828-35.

[77] Falcao-Silva, V. S.; Silva, D. A.; Souza Mde, F.; Siqueira-Junior, J. P., Modulation of drug resistance in Staphylococcus aureus by a kaempferol glycoside from Herissantia tiubae (Malvaceae). Phytother Res 2009, 23 (10), 1367-70.

[78] Holler, J. G.; Christensen, S. B.; Slotved, H. C.; Rasmussen, H. B.; Guzman, A.; Olsen, C. E.; Petersen, B.; Molgaard, P., Novel inhibitory activity of the Staphylococcus aureus NorA efflux pump by a kaempferol rhamnoside isolated from Persea lingue Nees. J Antimicrob Chemother 2012, 67 (5), 1138-44.

[79] Holler, J. G.; Slotved, H. C.; Molgaard, P.; Olsen, C. E.; Christensen, S. B., Chalcone inhibitors of the NorA efflux pump in Staphylococcus aureus whole cells and enriched 
everted membrane vesicles. Bioorg Med Chem 2012, 20 (14), 4514-21.

[80] Belofsky, G.; Percivill, D.; Lewis, K.; Tegos, G. P.; Ekart, J., Phenolic metabolites of Dalea versicolor that enhance antibiotic activity against model pathogenic bacteria. J Nat Prod 2004, 67 (3), 481-4.

[81] Stermitz, F. R.; Tawara-Matsuda, J.; Lorenz, P.; Mueller, P.; Zenewicz, L.; Lewis, K., 5'Methoxyhydnocarpin-D and pheophorbide A: Berberis species components that potentiate berberine growth inhibition of resistant Staphylococcus aureus. J Nat Prod 2000, 63 (8), 1146-9.

[82] Pereda-Miranda, R.; Kaatz, G. W.; Gibbons, S., Polyacylated oligosaccharides from medicinal Mexican morning glory species as antibacterials and inhibitors of multidrug resistance in Staphylococcus aureus. J Nat Prod 2006, 69 (3), 406-9.

[83] Cherigo, L.; Pereda-Miranda, R.; Fragoso-Serrano, M.; Jacobo-Herrera, N.; Kaatz, G. W.; Gibbons, S., Inhibitors of bacterial multidrug efflux pumps from the resin glycosides of Ipomoea murucoides. J Nat Prod 2008, 71 (6), 1037-45.

[84] Stermitz, F. R.; Cashman, K. K.; Halligan, K. M.; Morel, C.; Tegos, G. P.; Lewis, K., Polyacylated neohesperidosides From Geranium caespitosum: bacterial multidrug resistance pump inhibitors. Bioorg Med Chem Lett 2003, 13 (11), 1915-1918.

[85] Kalia, N. P.; Mahajan, P.; Mehra, R.; Nargotra, A.; Sharma, J. P.; Koul, S.; Khan, I. A., Capsaicin, a novel inhibitor of the NorA efflux pump, reduces the intracellular invasion of Staphylococcus aureus. J Antimicrob Chemother 2012, 67 (10), 2401-8.

[86] Fiamegos, Y. C.; Kastritis, P. L.; Exarchou, V.; Han, H.; Bonvin, A. M. J. J.; Vervoort, J.; Lewis, K.; Hamblin, M. R.; Tegos, G. P., Antimicrobial and Efflux Pump Inhibitory Activity of Caffeoylquinic Acids from Artemisia absinthium against Gram-Positive Pathogenic Bacteria. PLOS ONE 2011, 6 (4), e18127.

[87] Shiu, W. K.; Malkinson, J. P.; Rahman, M. M.; Curry, J.; Stapleton, P.; Gunaratnam, M.; Neidle, S.; Mushtaq, S.; Warner, M.; Livermore, D. M.; Evangelopoulos, D.; Basavannacharya, C.; Bhakta, S.; Schindler, B. D.; Seo, S. M.; Coleman, D.; Kaatz, G. W.; Gibbons, S., A new plantderived antibacterial is an inhibitor of efflux pumps in Staphylococcus aureus. Int $J$ Antimicrob Agents 2013, 42 (6), 513-8.

[88] Costa, L. M.; de Macedo, E. V.; Oliveira, F. A.; Ferreira, J. H.; Gutierrez, S. J.; Pelaez, W. J.; Lima, F. C.; de Siqueira Junior, J. P.; Coutinho, H. D.; Kaatz, G. W.; de Freitas, R. M.; Barreto, H. M., Inhibition of the NorA efflux pump of Staphylococcus aureus by synthetic riparins. $J$ Appl Microbiol 2016, 121 (5), 1312-1322.

[89] Michalet, S.; Cartier, G.; David, B.; Mariotte, A. M.; Dijoux-franca, M. G.; Kaatz, G. W.; Stavri, M.; Gibbons, S., $N$-caffeoylphenalkylamide derivatives as bacterial efflux pump inhibitors. Bioorg Med Chem Lett 2007, 17 (6), 17558.

[90] Abulrob, A. N.; Suller, M. T.; Gumbleton, M.; Simons, C.; Russell, A. D., Identification and biological evaluation of grapefruit oil components as potential novel efflux pump modulators in methicillin-resistant Staphylococcus aureus bacterial strains. Phytochemistry 2004, 65 (22), 3021-7. [91] Roy, S. K.; Kumari, N.; Pahwa, S.; Agrahari, U. C.; Bhutani, K. K.; Jachak, S. M.; Nandanwar, H., NorA efflux pump inhibitory activity of coumarins from Mesua ferrea. Fitoterapia 2013, 90, 140-50.

[92] Oliveira, A. S.; Sousa, E.; Vasconcelos, M. H.; Pinto, M., Curcumin: A Natural Lead for Potential New Drug Candidates. Curr Med Chem 2015, 22 (36), 4196-232.

[93] Lopes-Rodrigues, V.; Oliveira, A.; Correia-da-Silva, M.; Pinto, M.; Lima, R. T.; Sousa, E.; Vasconcelos, M. H., A novel curcumin derivative which inhibits P-glycoprotein, arrests cell cycle and induces apoptosis in multidrug resistance cells. Bioorg Med Chem 2017, 25 (2), 581-596.

[94] Joshi, P.; Singh, S.; Wani, A.; Sharma, S.; Jain, S. K.; Singh, B.; Gupta, B. D.; Satti, N. K.; Koul, S.; Khan, I. A.; Kumar, A.; Bharate, S. B.; Vishwakarma, R. A., Osthol and curcumin as inhibitors of human Pgp and multidrug efflux pumps of Staphylococcus aureus: reversing the resistance against frontline antibacterial drugs. Med Chem Comm 2014, 5 (10), 1540-1547.

[95] Marquez, B.; Neuville, L.; Moreau, N. J.; Genet, J. P.; dos Santos, A. F.; Cano de Andrade, M. C.; Sant'Ana, A. E., Multidrug resistance reversal agent from Jatropha elliptica. Phytochemistry 2005, 66 (15), 1804-11.

[96] Belofsky, G.; Carreno, R.; Lewis, K.; Ball, A.; Casadei, G.; Tegos, G. P., Metabolites of the "smoke tree", Dalea spinosa, potentiate antibiotic activity against multidrugresistant Staphylococcus aureus. J Nat Prod 2006, 69 (2), 261-4.

[97] Ambrus, J. I.; Kelso, M. J.; Bremner, J. B.; Ball, A. R.; Casadei, G.; Lewis, K., Structure-activity relationships of 2aryl-1H-indole inhibitors of the NorA efflux pump in Staphylococcus aureus. Bioorg Med Chem Lett 2008, 18 (15), 4294-7.

[98] Samosorn, S.; Bremner, J. B.; Ball, A.; Lewis, K., Synthesis of functionalized 2-aryl-5-nitro- $1 H$-indoles and their activity as bacterial NorA efflux pump inhibitors. Bioorg Med Chem 2006, 14 (3), 857-65.

[99] Ball, A. R.; Casadei, G.; Samosorn, S.; Bremner, J. B.; Ausubel, F. M.; Moy, T. I.; Lewis, K., Conjugating berberine to a multidrug efflux pump inhibitor creates an effective antimicrobial. ACS Chem Biol 2006, 1 (9), 594-600.

[100] Fournier dit Chabert, J.; Marquez, B.; Neville, L.; Joucla, L.; Broussous, S.; Bouhours, P.; David, E.; PelletRostaing, S.; Marquet, B.; Moreau, N.; Lemaire, M., Synthesis and evaluation of new arylbenzo $[b]$ thiophene and diarylthiophene derivatives as inhibitors of the NorA multidrug transporter of Staphylococcus aureus. Bioorg Med Chem 2007, 15 (13), 4482-4497.

[101] Samosorn, S.; Tanwirat, B.; Muhamad, N.; Casadei, G.; Tomkiewicz, D.; Lewis, K.; Suksamrarn, A.; Prammananan, T.; Gornall, K. C.; Beck, J. L.; Bremner, J. B., Antibacterial activity of berberine-NorA pump inhibitor hybrids with a methylene ether linking group. Bioorg Med Chem 2009, 17 (11), 3866-72. 
[102] Buonerba, F.; Lepri, S.; Goracci, L.; Schindler, B. D.; Seo, S. M.; Kaatz, G. W.; Cruciani, G., Improved Potency of Indole-Based NorA Efflux Pump Inhibitors: From Serendipity toward Rational Design and Development. $J$ Med Chem 2017, 60 (1), 517-523.

[103] Lepri, S.; Buonerba, F.; Goracci, L.; Velilla, I.; Ruzziconi, R.; Schindler, B. D.; Seo, S. M.; Kaatz, G. W.; Cruciani, G., Indole Based Weapons to Fight Antibiotic Resistance: A Structure-Activity Relationship Study. J Med Chem 2016, 59 (3), 867-91.

[104] German, N.; Wei, P.; Kaatz, G. W.; Kerns, R. J., Synthesis and evaluation of fluoroquinolone derivatives as substrate-based inhibitors of bacterial efflux pumps. Eur $J$ Med Chem 2008, 43 (11), 2453-63.

[105] Pieroni, M.; Dimovska, M.; Brincat, J. P.; Sabatini, S.; Carosati, E.; Massari, S.; Kaatz, G. W.; Fravolini, A., From 6-aminoquinolone antibacterials to 6-amino-7thiopyranopyridinylquinolone ethyl esters as inhibitors of Staphylococcus aureus multidrug efflux pumps. J Med Chem 2010, 53 (11), 4466-80.

[106] Sabatini, S.; Gosetto, F.; Manfroni, G.; Tabarrini, O.; Kaatz, G. W.; Patel, D.; Cecchetti, V., Evolution from a natural flavones nucleus to obtain 2-(4Propoxyphenyl)quinoline derivatives as potent inhibitors of the $S$. aureus NorA efflux pump. J Med Chem 2011, 54 (16), 5722-36.

[107] Sabatini, S.; Gosetto, F.; Iraci, N.; Barreca, M. L.; Massari, S.; Sancineto, L.; Manfroni, G.; Tabarrini, O.; Dimovska, M.; Kaatz, G. W.; Cecchetti, V., Re-evolution of the 2-phenylquinolines: ligand-based design, synthesis, and biological evaluation of a potent new class of Staphylococcus aureus NorA efflux pump inhibitors to combat antimicrobial resistance. J Med Chem 2013, 56 (12), 4975-89.

[108] Thota, N.; Reddy, M. V.; Kumar, A.; Khan, I. A.; Sangwan, P. L.; Kalia, N. P.; Koul, J. L.; Koul, S., Substituted dihydronaphthalenes as efflux pump inhibitors of Staphylococcus aureus. Eur J Med Chem 2010, 45 (9), 360716.

[109] Fontaine, F.; Hequet, A.; Voisin-Chiret, A. S.; Bouillon, A.; Lesnard, A.; Cresteil, T.; Jolivalt, C.; Rault, S., First identification of boronic species as novel potential inhibitors of the Staphylococcus aureus NorA efflux pump. $J$ Med Chem 2014, 57 (6), 2536-48.

[110] Fontaine, F.; Hequet, A.; Voisin-Chiret, A. S.; Bouillon, A.; Lesnard, A.; Cresteil, T.; Jolivalt, C.; Rault, S., Boronic species as promising inhibitors of the Staphylococcus aureus NorA efflux pump: study of 6substituted pyridine-3-boronic acid derivatives. Eur $\mathrm{J}$ Med Chem 2015, 95, 185-98.

[111] Brincat, J. P.; Carosati, E.; Sabatini, S.; Manfroni, G.; Fravolini, A.; Raygada, J. L.; Patel, D.; Kaatz, G. W.; Cruciani, G., Discovery of novel inhibitors of the NorA multidrug transporter of Staphylococcus aureus. J Med Chem 2011, 54 (1), 354-65.

[112] Vidaillac, C.; Guillon, J.; Arpin, C.; Forfar-Bares, I.; Ba, B. B.; Grellet, J.; Moreau, S.; Caignard, D. H.; Jarry, C.; Quentin, C., Synthesis of omeprazole analogues and evaluation of these as potential inhibitors of the multidrug efflux pump NorA of Staphylococcus aureus. Antimicrob Agents Chemother 2007, 51 (3), 831-8.

[113] Dalton, W. S.; Grogan, T. M.; Meltzer, P. S.; Scheper, R. J.; Durie, B. G.; Taylor, C. W.; Miller, T. P.; Salmon, S. E., Drug-resistance in multiple myeloma and non-Hodgkin's lymphoma: detection of P-glycoprotein and potential circumvention by addition of verapamil to chemotherapy. $J$ Clin Oncol 1989, 7 (4), 415-24.

[114] Aeschlimann, J. R.; Dresser, L. D.; Kaatz, G. W.; Rybak, M. J., Effects of NorA inhibitors on in vitro antibacterial activities and postantibiotic effects of levofloxacin, ciprofloxacin, and norfloxacin in genetically related strains of Staphylococcus aureus. Antimicrob Agents Chemother 1999, 43 (2), 335-40.

[115] Ng, E. Y.; Trucksis, M.; Hooper, D. C., Quinolone resistance mediated by nor $A$ : physiologic characterization and relationship to $f l q B$, a quinolone resistance locus on the Staphylococcus aureus chromosome. Antimicrob Agents Chemother 1994, 38 (6), 1345-55.

[116] Vidaillac, C.; Guillon, J.; Moreau, S.; Arpin, C.; Lagardere, A.; Larrouture, S.; Dallemagne, P.; Caignard, D. H.; Quentin, C.; Jarry, C., Synthesis of new 4-[2(alkylamino) ethylthio]pyrrolo[1,2-a]quinoxaline and 5-[2(alkylamino) ethylthio]pyrrolo[1,2-a]thieno[3,2-e]pyrazine derivatives, as potential bacterial multidrug resistance pump inhibitors. J Enzyme Inhib Med Chem 2007, 22 (5), 620-31. [117] Mullin, S.; Mani, N.; Grossman, T. H., Inhibition of Antibiotic Efflux in Bacteria by the Novel Multidrug Resistance Inhibitors Biricodar (VX-710) and Timcodar (VX-853). Antimicrob Agents Chemother 2004, 48 (11), 4171-4176.

[118] Bharate, J. B.; Singh, S.; Wani, A.; Sharma, S.; Joshi, P.; Khan, I. A.; Kumar, A.; Vishwakarma, R. A.; Bharate, S. B., Discovery of 4-acetyl-3-(4-fluorophenyl)-1-( $p$-tolyl)-5methylpyrrole as a dual inhibitor of human P-glycoprotein and Staphylococcus aureus NorA efflux pump. Org Biomol Chem 2015, 13 (19), 5424-31.

[119] Leitner, I.; Nemeth, J.; Feurstein, T.; Abrahim, A.; Matzneller, P.; Lagler, H.; Erker, T.; Langer, O.; Zeitlinger, M., The third-generation P-glycoprotein inhibitor tariquidar may overcome bacterial multidrug resistance by increasing intracellular drug concentration. J Antimicrob Chemother 2011, 66 (4), 834-9.

[120] Kaatz, G. W.; Moudgal, V. V.; Seo, S. M.; Hansen, J. B.; Kristiansen, J. E., Phenylpiperidine selective serotonin reuptake inhibitors interfere with multidrug efflux pump activity in Staphylococcus aureus. Int J Antimicrob Agents 2003, 22 (3), 254-61.

[121] Wei, P.; Kaatz, G. W.; Kerns, R. J., Structural differences between paroxetine and femoxetine responsible for differential inhibition of Staphylococcus aureus efflux pumps. Bioorg Med Chem Lett 2004, 14 (12), 3093-7.

[122] German, N.; Kaatz, G. W.; Kerns, R. J., Synthesis and evaluation of PSSRI-based inhibitors of Staphylococcus aureus multidrug efflux pumps. Bioorg Med Chem Lett 2008, 18 (4), 1368-73. 
[123] Kalle, A. M.; Rizvi, A., Inhibition of Bacterial Multidrug Resistance by Celecoxib, a Cyclooxygenase-2 Inhibitor. Antimicrob Agents Chemother 2011, 55 (1), 439442.

[124] Sabatini, S.; Gosetto, F.; Serritella, S.; Manfroni, G.; Tabarrini, O.; Iraci, N.; Brincat, J. P.; Carosati, E.; Villarini, M.; Kaatz, G. W.; Cecchetti, V., Pyrazolo[4,3c] $[1,2]$ benzothiazines 5,5-dioxide: a promising new class of Staphylococcus aureus NorA efflux pump inhibitors. $J$ Med Chem 2012, 55 (7), 3568-72.

[125] Kaatz, G. W.; Moudgal, V. V.; Seo, S. M.; Kristiansen, J. E., Phenothiazines and thioxanthenes inhibit multidrug efflux pump activity in Staphylococcus aureus. Antimicrob Agents Chemother 2003, 47 (2), 719-26.

[126] Sabatini, S.; Kaatz, G. W.; Rossolini, G. M.; Brandini, D.; Fravolini, A., From phenothiazine to 3-phenyl-1,4benzothiazine derivatives as inhibitors of the Staphylococcus aureus NorA multidrug efflux pump. J Med Chem 2008, 51 (14), 4321-30.

[127] Kristiansen, M. M.; Leandro, C.; Ordway, D.; Martins, M.; Viveiros, M.; Pacheco, T.; Molnar, J.; Kristiansen, J. E.; Amaral, L., Thioridazine reduces resistance of methicillinresistant Staphylococcus aureus by inhibiting a reserpinesensitive efflux pump. In Vivo 2006, 20 (3), 361-6.

[128] Kristiansen, J. E.; Hendricks, O.; Delvin, T.; Butterworth, T. S.; Aagaard, L.; Christensen, J. B.; Flores, V. C.; Keyzer, H., Reversal of resistance in microorganisms by help of non-antibiotics. J Antimicrob Chemother 2007, 59 (6), 1271-9.

[129] Alnaseri, H.; Arsic, B.; Schneider, J. E.; Kaiser, J. C.; Scinocca, Z. C.; Heinrichs, D. E.; McGavin, M. J., Inducible Expression of a Resistance-Nodulation-Division-Type Efflux Pump in Staphylococcus aureus Provides Resistance to Linoleic and Arachidonic Acids. J Bacteriol 2015, 197 (11), 1893-905.

[130] Schindler, B. D.; Frempong-Manso, E.; DeMarco, C. E.; Kosmidis, C.; Matta, V.; Seo, S. M.; Kaatz, G. W., Analyses of multidrug efflux pump-like proteins encoded on the Staphylococcus aureus chromosome. Antimicrob Agents Chemother 2015, 59 (1), 747-8.

[131] Murakami, S.; Nakashima, R.; Yamashita, E.; Yamaguchi, A., Crystal structure of bacterial multidrug efflux transporter AcrB. Nature 2002, 419 (6907), 587-93. [132] Kim, J.-S.; Jeong, H.; Song, S.; Kim, H.-Y.; Lee, K.; Hyun, J.; Ha, N.-C., Structure of the Tripartite Multidrug Efflux Pump AcrAB-TolC Suggests an Alternative Assembly Mode. Mol Cells 2015, 38 (2), 180-186.

[133] Du, D.; Wang, Z.; James, N. R.; Voss, J. E.; Klimont, E.; Ohene-Agyei, T.; Venter, H.; Chiu, W.; Luisi, B. F., Structure of the AcrAB-TolC multidrug efflux pump. Nature 2014, 509 (7501), 512-515.

[134] Pan, Y. P.; Xu, Y. H.; Wang, Z. X.; Fang, Y. P.; Shen, J. L., Overexpression of MexAB-OprM efflux pump in carbapenem-resistant Pseudomonas aeruginosa. Arch Microbiol 2016, 198 (6), 565-71.

[135] Lorenzi, V.; Muselli, A.; Bernardini, A. F.; Berti, L.; Pages, J. M.; Amaral, L.; Bolla, J. M., Geraniol restores antibiotic activities against multidrug-resistant isolates from
Gram-negative species. Antimicrob Agents Chemother 2009, 53 (5), 2209-11.

[136] Aparna, V.; Dineshkumar, K.; Mohanalakshmi, N.; Velmurugan, D.; Hopper, W., Identification of Natural Compound Inhibitors for Multidrug Efflux Pumps of Escherichia coli and Pseudomonas aeruginosa Using In Silico High-Throughput Virtual Screening and In Vitro Validation. PLOS ONE 2014, 9 (7), e101840.

[137] Ohene-Agyei, T.; Mowla, R.; Rahman, T.; Venter, H., Phytochemicals increase the antibacterial activity of antibiotics by acting on a drug efflux pump. Microbiologyopen 2014, 3 (6), 885-96.

[138] Negi, N.; Prakash, P.; Gupta, M. L.; Mohapatra, T. M., Possible Role of Curcumin as an Efflux Pump Inhibitor in Multi Drug Resistant Clinical Isolates of Pseudomonas aeruginosa. J Clin Diagn Res 2014, 8 (10), DC04-7.

[139] Lee, M. D.; Galazzo, J. L.; Staley, A. L.; Lee, J. C.; Warren, M. S.; Fuernkranz, H.; Chamberland, S.; Lomovskaya, O.; Miller, G. H., Microbial fermentationderived inhibitors of efflux-pump-mediated drug resistance. Farmaco 2001, 56 (1-2), 81-5.

[140] Nguyen, S. T.; Kwasny, S. M.; Ding, X.; Cardinale, S. C.; McCarthy, C. T.; Kim, H. S.; Nikaido, H.; Peet, N. P.; Williams, J. D.; Bowlin, T. L.; Opperman, T. J., Structureactivity relationships of a novel pyranopyridine series of Gram-negative bacterial efflux pump inhibitors. Bioorg Med Chem 2015, 23 (9), 2024-34.

[141] Opperman, T. J.; Kwasny, S. M.; Kim, H. S.; Nguyen, S. T.; Houseweart, C.; D'Souza, S.; Walker, G. C.; Peet, N. P.; Nikaido, H.; Bowlin, T. L., Characterization of a novel pyranopyridine inhibitor of the AcrAB efflux pump of Escherichia coli. Antimicrob Agents Chemother 2014, 58 (2), 722-33.

[142] Renau, T. E.; Leger, R.; Flamme, E. M.; Sangalang, J.; She, M. W.; Yen, R.; Gannon, C. L.; Griffith, D.; Chamberland, S.; Lomovskaya, O.; Hecker, S. J.; Lee, V. J.; Ohta, T.; Nakayama, K., Inhibitors of efflux pumps in Pseudomonas aeruginosa potentiate the activity of the fluoroquinolone antibacterial levofloxacin. $J$ Med Chem 1999, 42 (24), 4928-31.

[143] Lomovskaya, O.; Warren, M. S.; Lee, A.; Galazzo, J.; Fronko, R.; Lee, M.; Blais, J.; Cho, D.; Chamberland, S.; Renau, T.; Leger, R.; Hecker, S.; Watkins, W.; Hoshino, K.; Ishida, H.; Lee, V. J., Identification and characterization of inhibitors of multidrug resistance efflux pumps in Pseudomonas aeruginosa: novel agents for combination therapy. Antimicrob Agents Chemother 2001, 45 (1), 105-16. [144] Renau, T. E.; Leger, R.; Flamme, E. M.; She, M. W.; Gannon, C. L.; Mathias, K. M.; Lomovskaya, O.; Chamberland, S.; Lee, V. J.; Ohta, T.; Nakayama, K.; Ishida, Y., Addressing the stability of C-capped dipeptide efflux pump inhibitors that potentiate the activity of levofloxacin in Pseudomonas aeruginosa. Bioorg Med Chem Lett 2001, 11 (5), 663-7.

[145] Renau, T. E.; Leger, R.; Filonova, L.; Flamme, E. M.; Wang, M.; Yen, R.; Madsen, D.; Griffith, D.; Chamberland, S.; Dudley, M. N.; Lee, V. J.; Lomovskaya, O.; Watkins, W. J.; Ohta, T.; Nakayama, K.; Ishida, Y., Conformationally- 
restricted analogues of efflux pump inhibitors that potentiate the activity of levofloxacin in Pseudomonas aeruginosa. Bioorg Med Chem Lett 2003, 13 (16), 2755-8.

[146] Renau, T. E.; Leger, R.; Yen, R.; She, M. W.; Flamme, E. M.; Sangalang, J.; Gannon, C. L.; Chamberland, S.; Lomovskaya, O.; Lee, V. J., Peptidomimetics of efflux pump inhibitors potentiate the activity of levofloxacin in Pseudomonas aeruginosa. Bioorg Med Chem Lett 2002, 12 (5), 763-6.

[147] Vargiu, A. V.; Ruggerone, P.; Opperman, T. J.; Nguyen, S. T.; Nikaido, H., Molecular mechanism of MBX2319 inhibition of Escherichia coli AcrB multidrug efflux pump and comparison with other inhibitors. Antimicrob Agents Chemother 2014, 58 (10), 6224-34.

[148] Sjuts, H.; Vargiu, A. V.; Kwasny, S. M.; Nguyen, S. T.; Kim, H. S.; Ding, X.; Ornik, A. R.; Ruggerone, P.; Bowlin, T. L.; Nikaido, H.; Pos, K. M.; Opperman, T. J., Molecular basis for inhibition of AcrB multidrug efflux pump by novel and powerful pyranopyridine derivatives. Proc Natl Acad Sci U S A 2016, 113 (13), 3509-14.

[149] Bohnert, J. A.; Kern, W. V., Selected arylpiperazines are capable of reversing multidrug resistance in Escherichia coli overexpressing RND efflux pumps. Antimicrob Agents Chemother 2005, 49 (2), 849-52.

[150] Kern, W. V.; Steinke, P.; Schumacher, A.; Schuster, S.; von Baum, H.; Bohnert, J. A., Effect of 1-(1naphthylmethyl)-piperazine, a novel putative efflux pump inhibitor, on antimicrobial drug susceptibility in clinical isolates of Escherichia coli. J Antimicrob Chemother 2006, 57 (2), 339-43.

[151] Opperman, T. J.; Nguyen, S. T., Recent advances toward a molecular mechanism of efflux pump inhibition. Front Microbiol 2015, 6, 421.

[152] Nakayama, K.; Ishida, Y.; Ohtsuka, M.; Kawato, H.; Yoshida, K.-i.; Yokomizo, Y.; Hosono, S.; Ohta, T.; Hoshino, K.; Ishida, H.; Yoshida, K.; Renau, T. E.; Léger, R.; Zhang, J. Z.; Lee, V. J.; Watkins, W. J., MexAB-OprMSpecific efflux pump inhibitors in Pseudomonas aeruginosa. Part 1: Discovery and early strategies for lead optimization. Bioorg Med Chem Lett 2003, 13 (23), 4201-4204.

[153] Nakayama, K.; Ishida, Y.; Ohtsuka, M.; Kawato, H.; Yoshida, K.; Yokomizo, Y.; Ohta, T.; Hoshino, K.; Otani, T.; Kurosaka, Y.; Yoshida, K.; Ishida, H.; Lee, V. J.; Renau, T. E.; Watkins, W. J., MexAB-OprM specific efflux pump inhibitors in Pseudomonas aeruginosa. Part 2: achieving activity in vivo through the use of alternative scaffolds. Bioorg Med Chem Lett 2003, 13 (23), 4205-8.

[154] Nakayama, K.; Kawato, H.; Watanabe, J.; Ohtsuka, M.; Yoshida, K.; Yokomizo, Y.; Sakamoto, A.; Kuru, N.; Ohta, T.; Hoshino, K.; Yoshida, K.; Ishida, H.; Cho, A.; Palme, M. H.; Zhang, J. Z.; Lee, V. J.; Watkins, W. J., MexAB-OprM specific efflux pump inhibitors in Pseudomonas aeruginosa. Part 3: Optimization of potency in the pyridopyrimidine series through the application of a pharmacophore model. Bioorg Med Chem Lett 2004, 14 (2), 475-9.

[155] Nakayama, K.; Kuru, N.; Ohtsuka, M.; Yokomizo, Y.; Sakamoto, A.; Kawato, H.; Yoshida, K.; Ohta, T.; Hoshino,
K.; Akimoto, K.; Itoh, J.; Ishida, H.; Cho, A.; Palme, M. H.; Zhang, J. Z.; Lee, V. J.; Watkins, W. J., MexAB-OprM specific efflux pump inhibitors in Pseudomonas aeruginosa. Part 4: Addressing the problem of poor stability due to photoisomerization of an acrylic acid moiety. Bioorg Med Chem Lett 2004, 14 (10), 2493-7.

[156] Yoshida, K.; Nakayama, K.; Kuru, N.; Kobayashi, S.; Ohtsuka, M.; Takemura, M.; Hoshino, K.; Kanda, H.; Zhang, J. Z.; Lee, V. J.; Watkins, W. J., MexAB-OprM specific efflux pump inhibitors in Pseudomonas aeruginosa. Part 5: Carbon-substituted analogues at the C-2 position. Bioorg Med Chem 2006, 14 (6), 1993-2004.

[157] Yoshida, K.; Nakayama, K.; Yokomizo, Y.; Ohtsuka, M.; Takemura, M.; Hoshino, K.; Kanda, H.; Namba, K.; Nitanai, H.; Zhang, J. Z.; Lee, V. J.; Watkins, W. J., MexAB-OprM specific efflux pump inhibitors in Pseudomonas aeruginosa. Part 6: exploration of aromatic substituents. Bioorg Med Chem 2006, 14 (24), 8506-18.

[158] Yoshida, K.; Nakayama, K.; Ohtsuka, M.; Kuru, N.; Yokomizo, Y.; Sakamoto, A.; Takemura, M.; Hoshino, K.; Kanda, H.; Nitanai, H.; Namba, K.; Yoshida, K.; Imamura, Y.; Zhang, J. Z.; Lee, V. J.; Watkins, W. J., MexAB-OprM specific efflux pump inhibitors in Pseudomonas aeruginosa. Part 7: highly soluble and in vivo active quaternary ammonium analogue D13-9001, a potential preclinical candidate. Bioorg Med Chem 2007, 15 (22), 7087-97.

[159] Mallea, M.; Mahamoud, A.; Chevalier, J.; AlibertFranco, S.; Brouant, P.; Barbe, J.; Pages, J. M., Alkylaminoquinolines inhibit the bacterial antibiotic efflux pump in multidrug-resistant clinical isolates. Biochem $J$ 2003, 376 (Pt 3), 801-5.

[160] Chevalier, J.; Bredin, J.; Mahamoud, A.; Mallea, M.; Barbe, J.; Pages, J. M., Inhibitors of antibiotic efflux in resistant Enterobacter aerogenes and Klebsiella pneumoniae strains. Antimicrob Agents Chemother 2004, 48 (3), 1043-6. [161] Ghisalberti, D.; Mahamoud, A.; Chevalier, J.; Baitiche, M.; Martino, M.; Pages, J. M.; Barbe, J., Chloroquinolines block antibiotic efflux pumps in antibiotic-resistant Enterobacter aerogenes isolates. Int J Antimicrob Agents 2006, 27 (6), 565-9.

[162] Mahamoud, A.; Chevalier, J.; Baitiche, M.; Adam, E.; Pages, J. M., An alkylaminoquinazoline restores antibiotic activity in Gram-negative resistant isolates. Microbiology 2011, 157 (Pt 2), 566-71.

[163] Yilmaz, S.; Altinkanat-Gelmez, G.; Bolelli, K.; Guneser-Merdan, D.; Over-Hasdemir, M. U.; Yildiz, I.; AkiYalcin, E.; Yalcin, I., Pharmacophore generation of 2substituted benzothiazoles as AdeABC efflux pump inhibitors in A. baumannii. SAR QSAR Environ Res 2014, 25 (7), 551-63.

[164] Yilmaz, S.; Altinkanat-Gelmez, G.; Bolelli, K.; Guneser-Merdan, D.; Ufuk Over-Hasdemir, M.; Aki-Yalcin, E.; Yalcin, I., Binding site feature description of 2substituted benzothiazoles as potential AcrAB-TolC efflux pump inhibitors in E. coli. SAR QSAR Environ Res 2015, 26 (10), 853-71.

[165] Zeng, B.; Wang, H.; Zou, L.; Zhang, A.; Yang, X.; Guan, Z., Evaluation and target validation of indole 
derivatives as inhibitors of the AcrAB-TolC efflux pump. Biosci Biotechnol Biochem 2010, 74 (11), 2237-41.

[166] Bohnert, J. A.; Szymaniak-Vits, M.; Schuster, S.; Kern, W. V., Efflux inhibition by selective serotonin reuptake inhibitors in Escherichia coli. $J$ Antimicrob Chemother 2011, 66 (9), 2057-60.

[167] Piddock, L. J.; Garvey, M. I.; Rahman, M. M.; Gibbons, S., Natural and synthetic compounds such as trimethoprim behave as inhibitors of efflux in Gram-negative bacteria. J Antimicrob Chemother 2010, 65 (6), 1215-23. [168] Li, B.; Yao, Q.; Pan, X. C.; Wang, N.; Zhang, R.; Li, J.; Ding, G.; Liu, X.; Wu, C.; Ran, D.; Zheng, J.; Zhou, H., Artesunate enhances the antibacterial effect of $\beta$-lactam antibiotics against Escherichia coli by increasing antibiotic accumulation via inhibition of the multidrug efflux pump system AcrAB-TolC. J Antimicrob Chemother 2011, 66 (4), 769-77.

[169] Bay, D. C.; Rommens, K. L.; Turner, R. J., Small multidrug resistance proteins: A multidrug transporter family that continues to grow. Biochim Biophys Acta 2008, 1778 (9), 1814-1838.

[170] Chen, Y.-J.; Pornillos, O.; Lieu, S.; Ma, C.; Chen, A. P.; Chang, G., X-ray structure of EmrE supports dual topology model. Proc Natl Acad Sci 2007, 104 (48), 1899919004.

[171] Suriyanarayanan, B.; Sarojini Santhosh, R., Docking analysis insights quercetin can be a non-antibiotic adjuvant by inhibiting Mmr drug efflux pump in Mycobacterium sp. and its homologue EmrE in Escherichia coli. J Biomol Struct Dyn 2015, 33 (8), 1819-34.

[172] Malkhed, V.; Mustyala, K. K.; Potlapally, S. R.; Vuruputuri, U., Identification of novel leads applying in silico studies for Mycobacterium multidrug resistant (MMR) protein. J Biomol Struct Dyn 2014, 32 (12), 1889-906.

[173] van Veen, H. W., Structural biology: Last of the multidrug transporters. Nature 2010, 467 (7318), 926-927.

[174] Tsuda, M.; Terada, T.; Asaka, J.; Ueba, M.; Katsura, $\mathrm{T}$.; Inui, K., Oppositely directed $\mathrm{H}+$ gradient functions as a driving force of rat $\mathrm{H}+/$ organic cation antiporter MATE1. Am J Physiol Renal Physiol 2007, 292 (2), F593-8.

[175] Terada, T.; Inui, K., Physiological and pharmacokinetic roles of $\mathrm{H}+$ /organic cation antiporters (MATE/SLC47A). Biochem Pharmacol 2008, 75 (9), 168996.

[176] Tanaka, Y.; Hipolito, C. J.; Maturana, A. D.; Ito, K.; Kuroda, T.; Higuchi, T.; Katoh, T.; Kato, H. E.; Hattori, M.; Kumazaki, K.; Tsukazaki, T.; Ishitani, R.; Suga, H.; Nureki, O., Structural basis for the drug extrusion mechanism by a MATE multidrug transporter. Nature 2013, 496 (7444), 24751.

[177] Radchenko, M.; Symersky, J.; Nie, R.; Lu, M., Structural basis for the blockade of MATE multidrug efflux pumps. Nat Commun 2015, 6, 7995.

[178] Omote, H.; Hiasa, M.; Matsumoto, T.; Otsuka, M.; Moriyama, Y., The MATE proteins as fundamental transporters of metabolic and xenobiotic organic cations. Trends Pharmacol Sci 2006, 27 (11), 587-93.
[179] Moriyama, Y.; Hiasa, M.; Matsumoto, T.; Omote, H., Multidrug and toxic compound extrusion (MATE)-type proteins as anchor transporters for the excretion of metabolic waste products and xenobiotics. Xenobiotica 2008, 38 (7-8), 1107-18.

[180] Kuroda, T.; Tsuchiya, T., Multidrug efflux transporters in the MATE family. Biochim Biophys Acta 2009, 1794 (5), 763-8.

[181] Du, D.; van Veen, H. W.; Murakami, S.; Pos, K. M.; Luisi, B. F., Structure, mechanism and cooperation of bacterial multidrug transporters. Curr Opin Struct Biol 2015, $33,76-91$.

[182] Kesherwani, M.; Michael Gromiha, M.; Fukui, K.; Velmurugan, D., Identification of novel natural inhibitor for NorM - a multidrug and toxic compound extrusion transporter - an in silico molecular modeling and simulation studies. J Biomol Struct Dyn 2017, 35 (1), 58-77. 


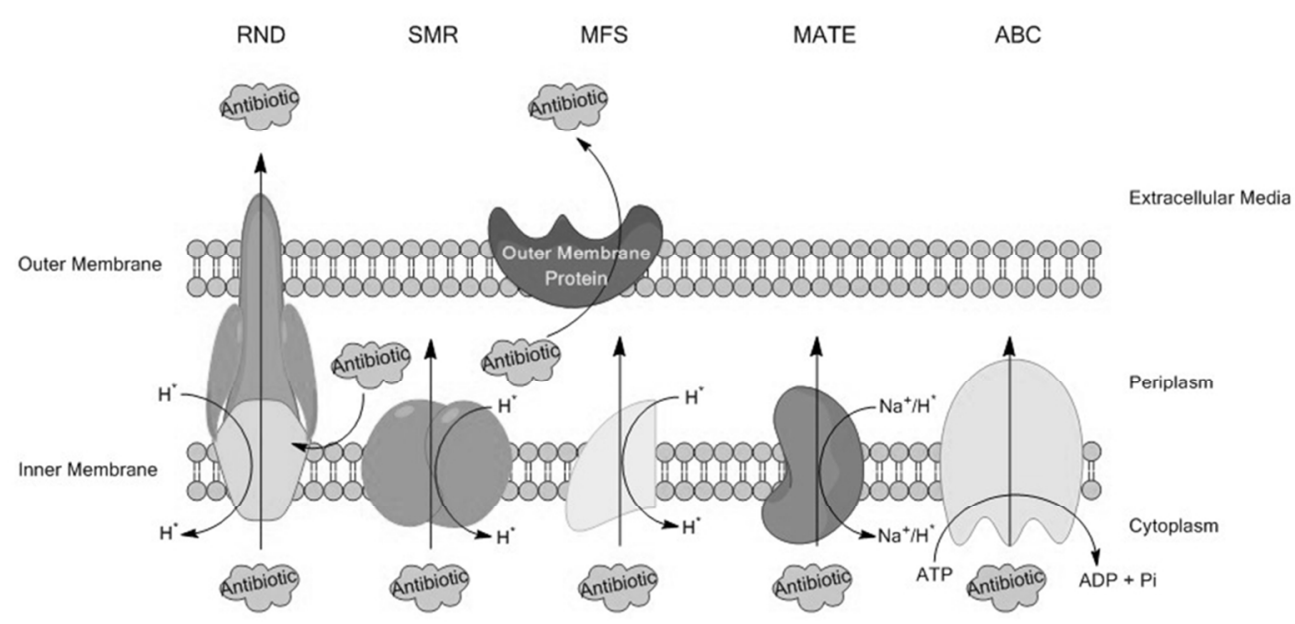

Figure 1 Representation of the five efflux pump families (adapted from [8]).

$307 \times 147 m m(96 \times 96$ DPI) 
Figure 2 Crystal structure of MsrA, from Mycobacterium tuberculosis in complex with protein-bound
methionine (PDB 1NWA).

Figure 2 Crystal structure of MsrA, from Mycobacterium tuberculosis in complex with protein-bound
methionine (PDB 1NWA). $92 \times 119 \mathrm{~mm}(96 \times 96 \mathrm{DPI})$

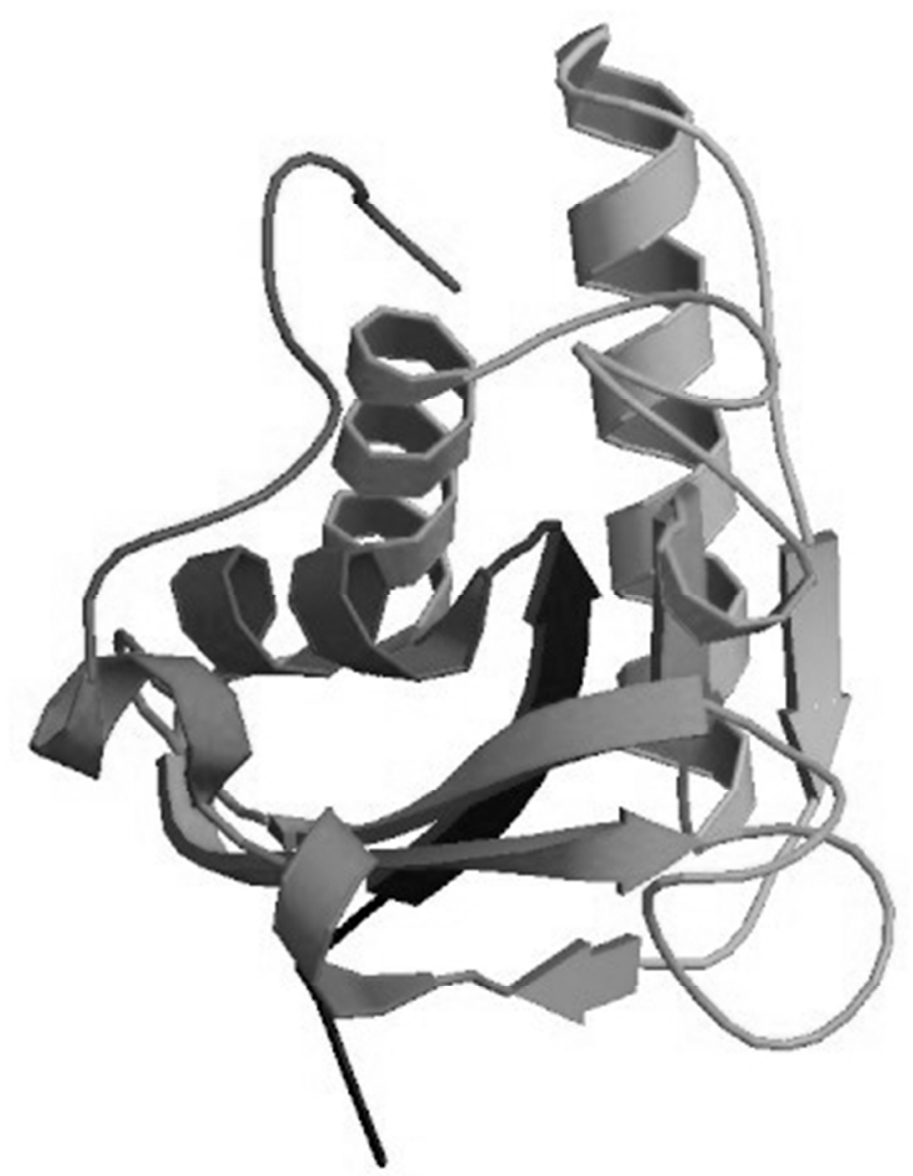




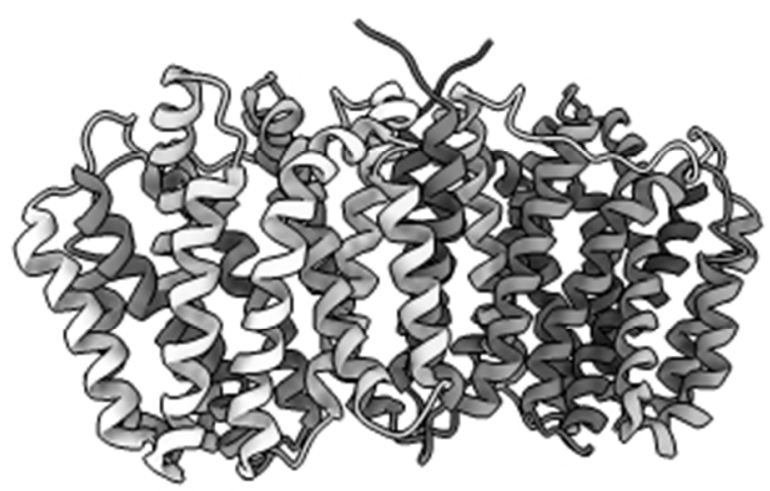

Figure 4 Crystal structure of EmrD, from E. coli (PDB 2GFP).

$82 \times 54 \mathrm{~mm}(96 \times 96 \mathrm{DPI})$ 
2-Hydroxymethyl-aniline, metaaminobenzonitrile and phenylacetamide show potentiation of ciprofloxacin

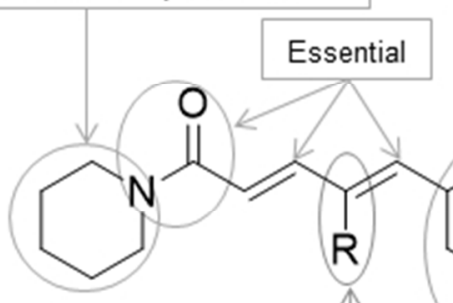

31

(Di-)lsopropyl and isobutyl $\rightarrow$ maximum potentiation of ciprofloxacin
Retention of 3,4-methylenedioxyphenyl or 4-methoxyphenyl gave favorable

Figure 6 Conclusions from SAR studies for piperine and its derivatives.

$156 \times 91 \mathrm{~mm}(96 \times 96 \mathrm{DPI})$ 


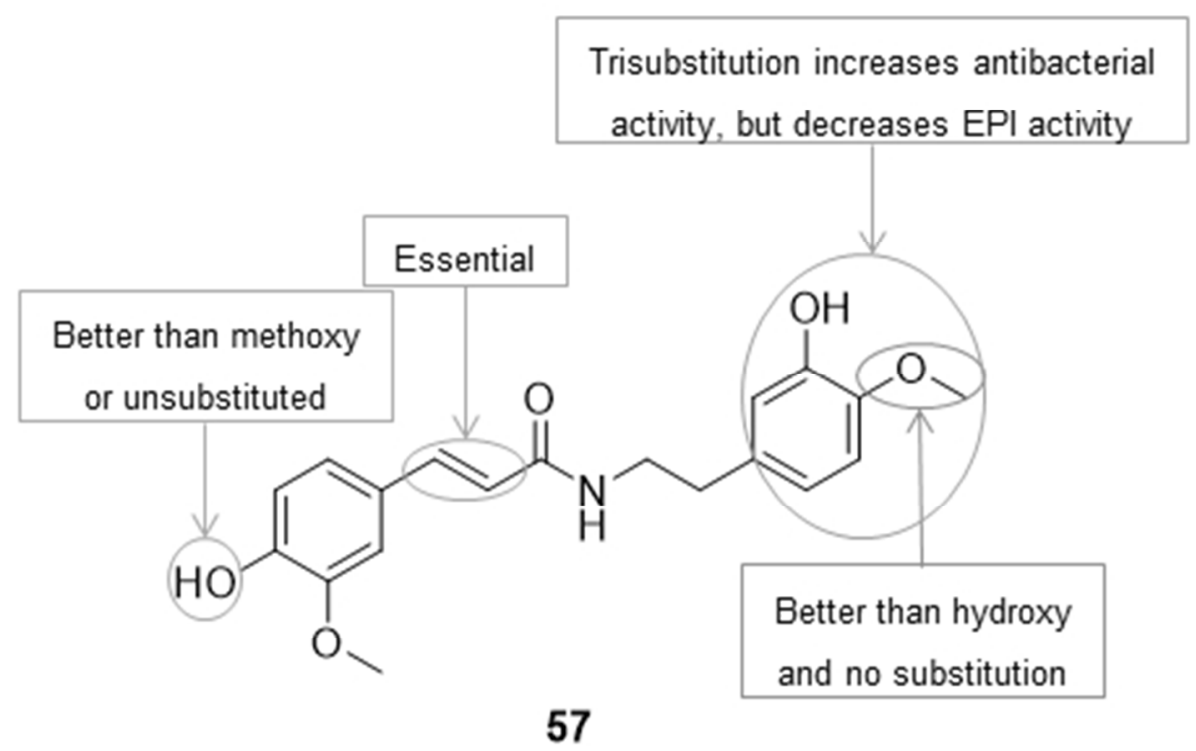

Figure 7 Conclusions from SAR studies for compound 57. $124 \times 80 \mathrm{~mm}(96 \times 96 \mathrm{DPI})$ 


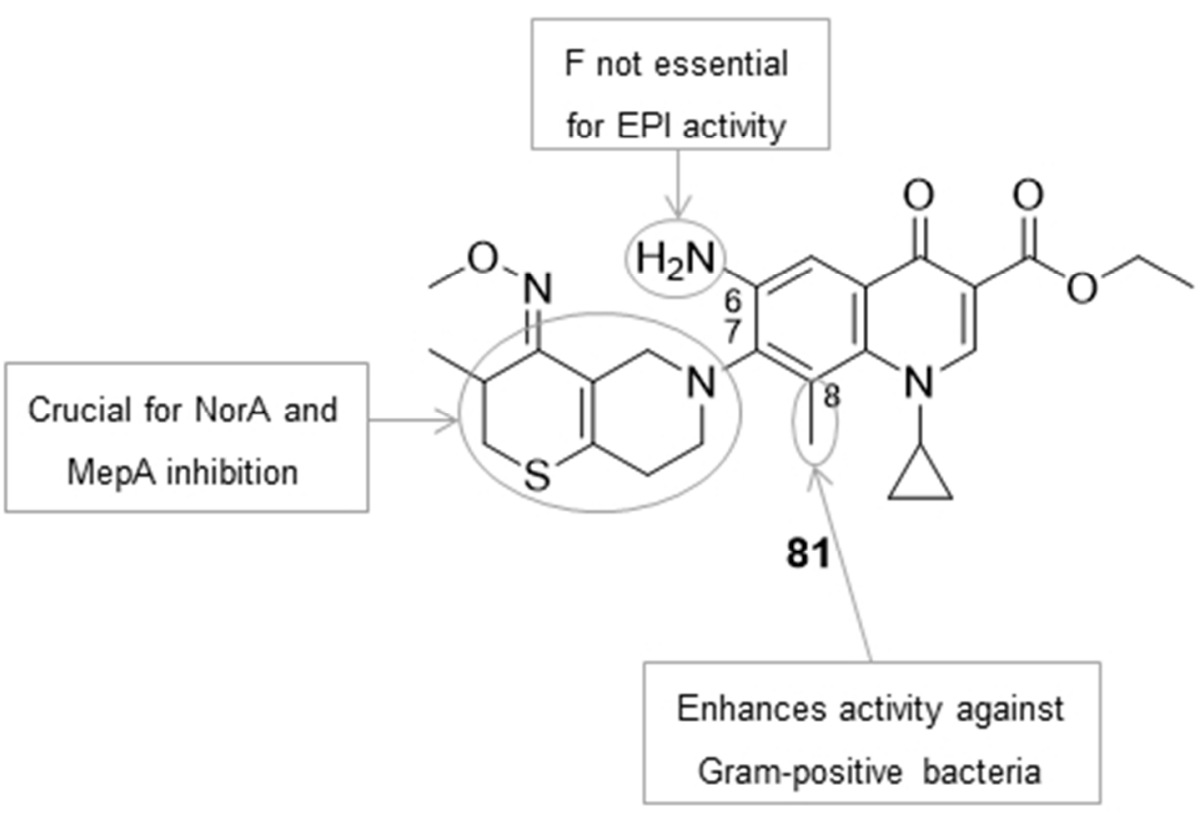

Figure 8 Conclusions from SAR studies for fluoroquinolone 81 derivatives.

$124 \times 84 \mathrm{~mm}(96 \times 96 \mathrm{DPI})$ 

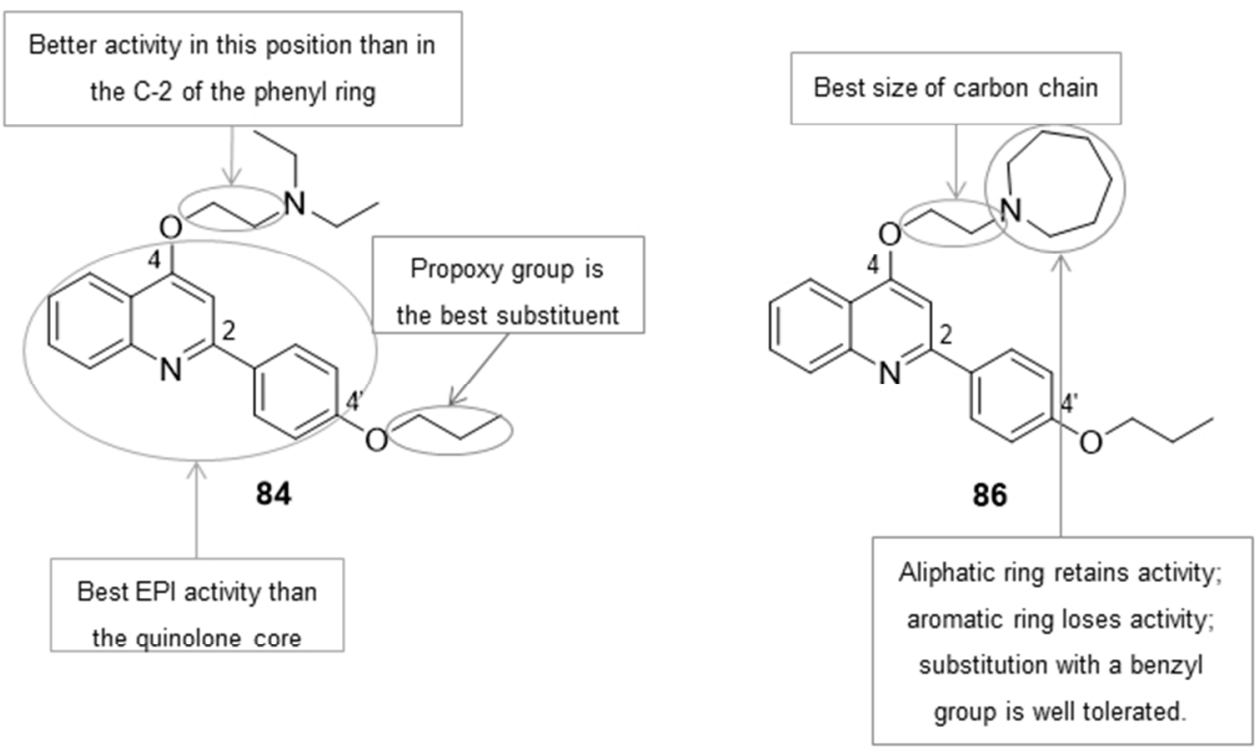

Figure 9 Conclusions from SAR studies for 2-phenyl-4-hydroxyquinoline derivatives 84 and 86. $177 \times 106 \mathrm{~mm}(96 \times 96$ DPI) 
Restores norfloxacin's bactericidal activity

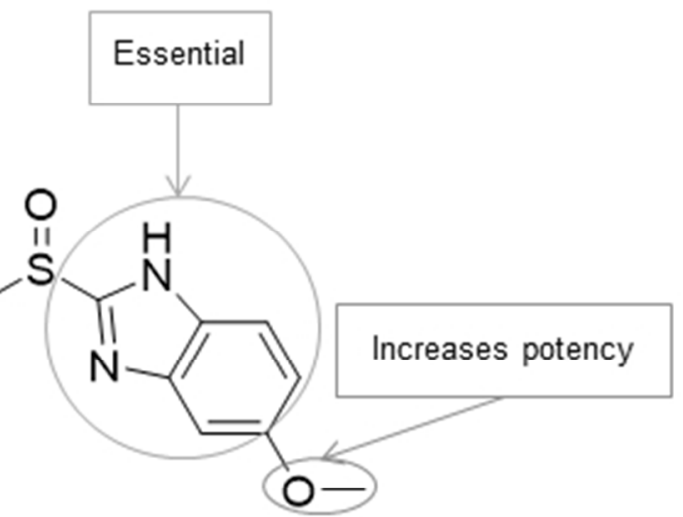

\section{7}

Figure 10 Conclusions from SAR studies for omeprazole derivatives.

$141 \times 69 \mathrm{~mm}(96 \times 96 \mathrm{DPI})$ 
Figure 11 Conclusions from SAR studies for paroxetine derivatives. $109 \times 111 \mathrm{~mm}(96 \times 96 \mathrm{DPI})$ 

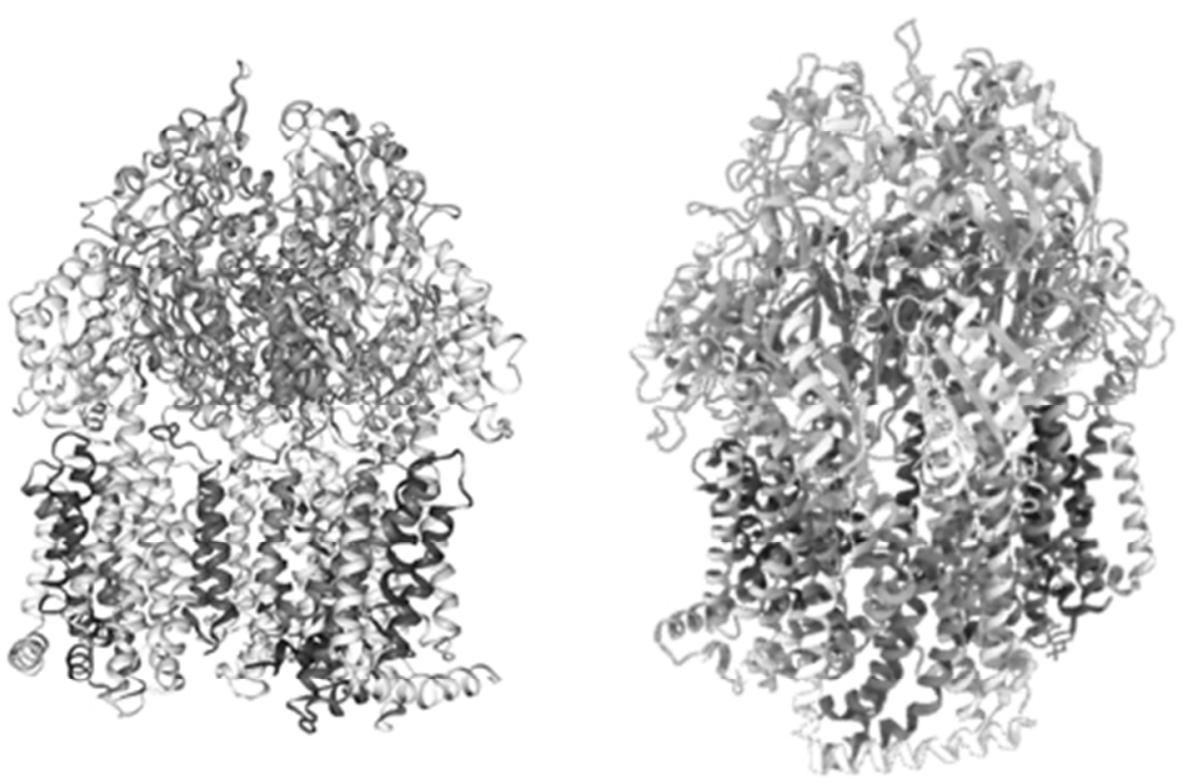

Figure 12 Crystal structures of AcrB (left, PDB 4ZLJ) of E. coli and MexB (right, PDB 2V50) of P. aeruginosa. $128 \times 83 \mathrm{~mm}(96 \times 96 \mathrm{DPI})$ 


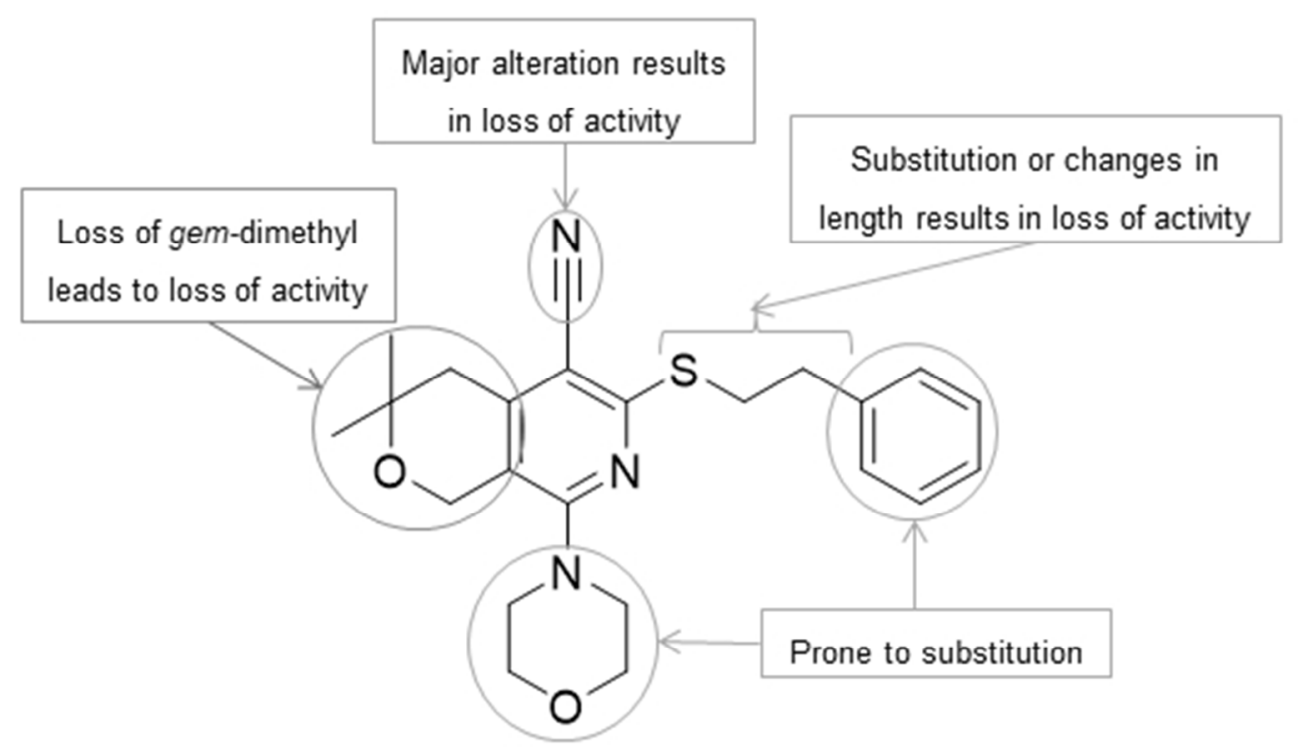

Figure 14 Conclusions from SAR studies for MBX2319 (127) (adapted from [28]). $142 \times 88 \mathrm{~mm}(96 \times 96 \mathrm{DPI})$ 


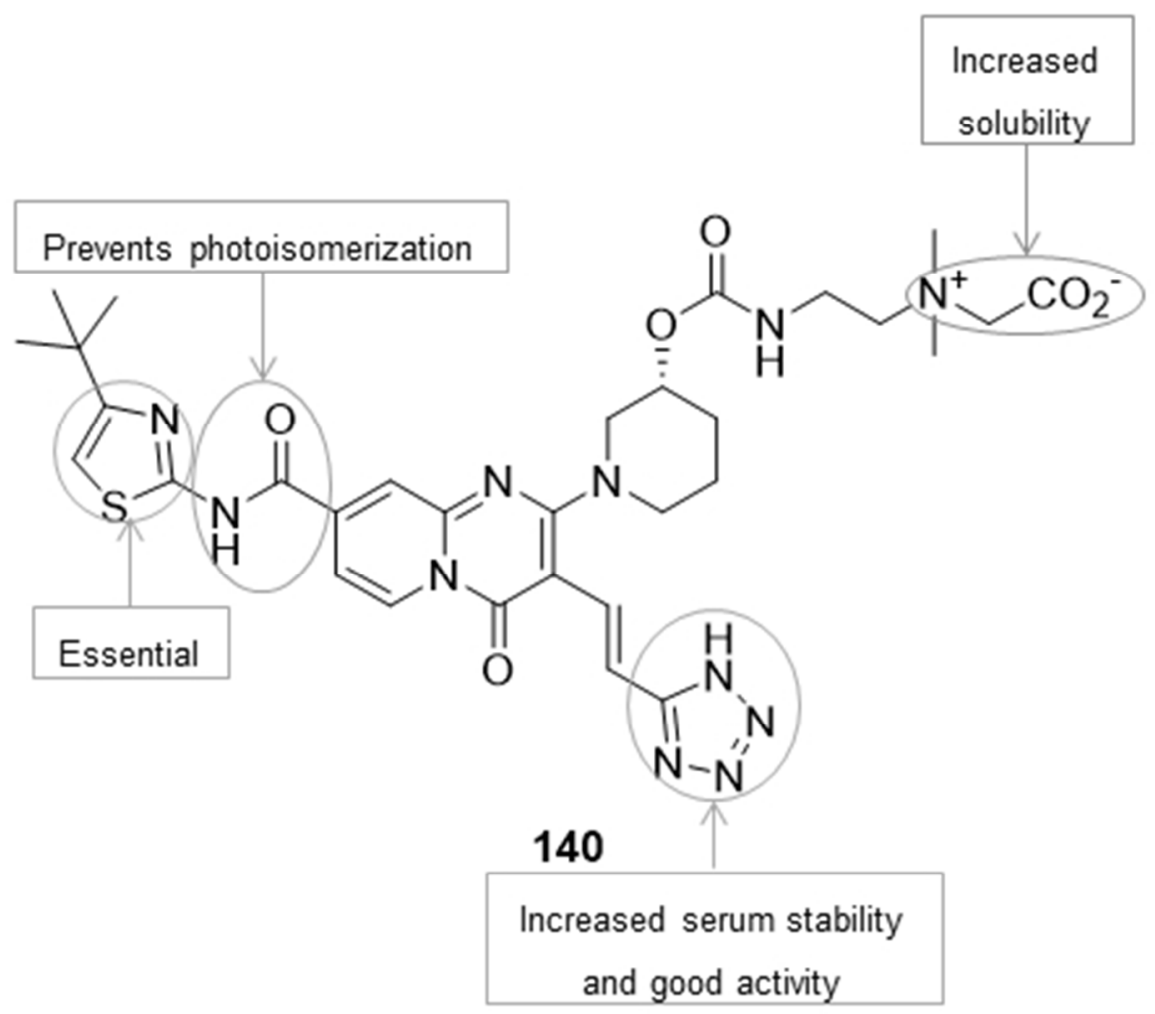

Figure 15 Conclusions from SAR studies for pyridopyrimidine 140 and analogues.

$122 \times 106 \mathrm{~mm}(96 \times 96 \mathrm{DPI})$ 


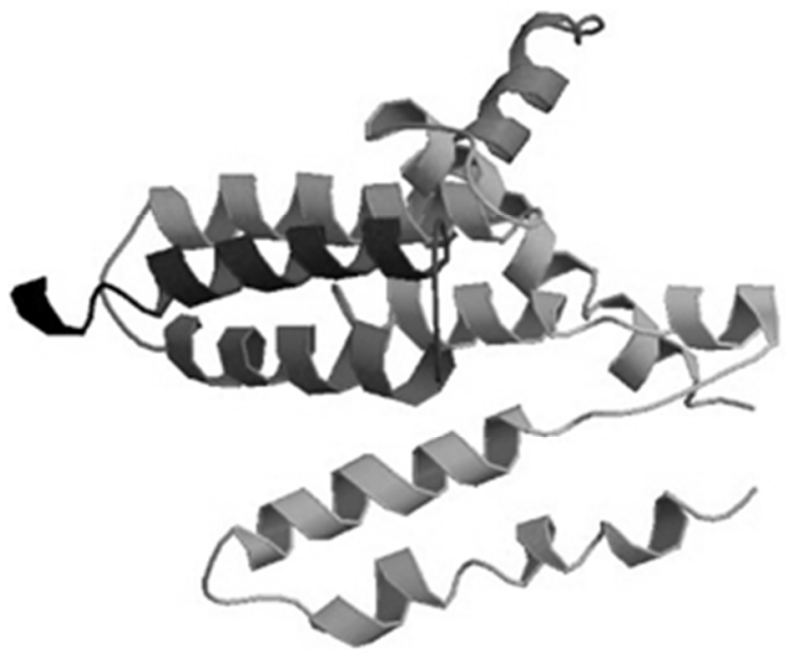

Figure 16 Crystal structure of EmrE (PDB 3B61) of E. coli. $83 \times 67 \mathrm{~mm}(96 \times 96$ DPI $)$ 
Figure 18 Crystal structure of MepA (PDB 1TZP) of E. coli in its inactive form.

$$
53 \times 45 \mathrm{~mm}(96 \times 96 \mathrm{DPI})
$$

